\title{
Identification and Characterization of Protein Complexes Involved in Different Pain States in Vertebrates
}

\author{
Dissertation \\ for the award of the degree \\ Doctor rerum naturalium \\ (Dr.rer.nat.) \\ of the Georg-August-Universität Göttingen \\ within the doctoral program \\ International Max Planck Research School for Neurosciences \\ of the Georg-August University School of Science (GAUSS) \\ submitted by \\ Julia Regina Sondermann \\ from Bochum, \\ Germany
}

Göttingen 2017 
Thesis Advisory Committee \& Members of the Examination Board

Dr. Manuela Schmidt (1 ${ }^{\text {st }}$ Referee)

Somatosensory Signaling \& Systems Biology Group

Max-Planck-Institute for Experimental Medicine, Göttingen

Prof. Dr. Martin C. Göpfert ( $2^{\text {nd }}$ Referee)

Department of Cellular Neurobiology

Schwann-Schleiden Research Centre, Georg-August-Universität, Göttingen

Prof. Dr. Henning Urlaub

Bioanalytical Mass Spectrometry Group

Max-Planck-Institute for Biophysical Chemistry, Göttingen

Further members of the Examination Board

Camin Dean, Ph.D.

Trans-synaptic Signaling Group

European Neuroscience Institute, Göttingen

Prof. Dr. Thomas Dresbach

Department of Anatomy

University Medical Centre, Göttingen

Prof. Dr. Michael W. Sereda

Molecular and Translational Neurology Group

Max-Planck-Institute for Experimental Medicine, Göttingen

Date of oral examination: $29^{\text {th }}$ November 2017 


\section{Declaration}

I hereby declare that the Ph.D. thesis entitled "Identification and Characterization of Protein Complexes Involved in Different Pain States in Vertebrates" was written independently and with no other sources and aids than quoted.

Göttingen, February 2019 


\section{Table of Contents}

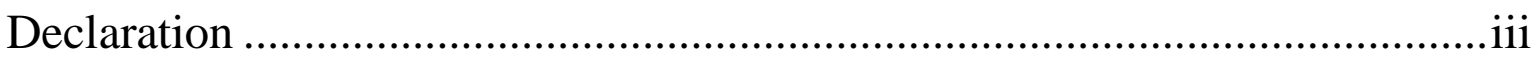

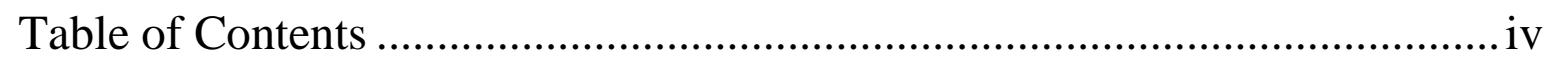

List of Figures ..................................................................................

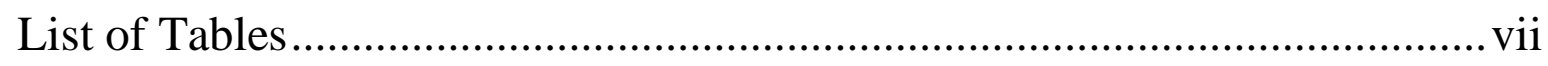

List of Abbreviations.........................................................................................

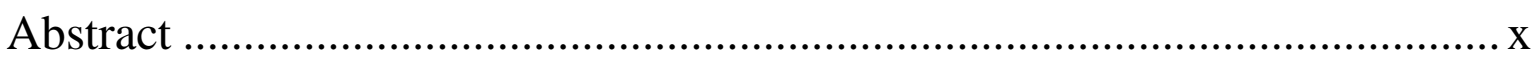

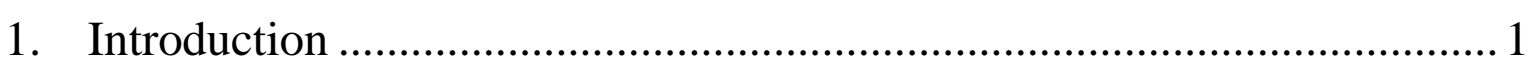

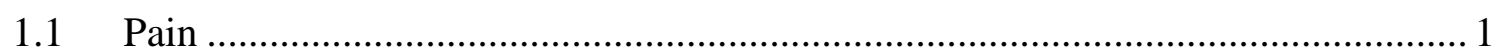

1.2 Nociception and pain - from the detection of noxious stimuli in the periphery to the sensation of pain .................................................................................. 1

1.3 Sensitization of the nociceptor - paving the way for pathological pain ................. 4

1.3.1 Peripheral sensitization - functional changes in nociceptors .......................... 5

1.4 TRP ion channels - polymodal signal integrators ................................................... 6

1.4.1 TRP channels - transducer of nociceptive stimuli ......................................... 8

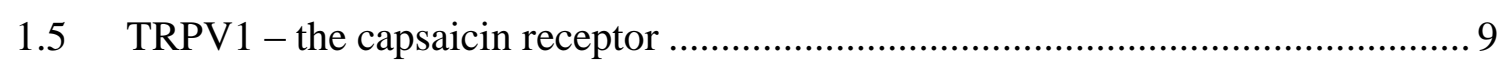

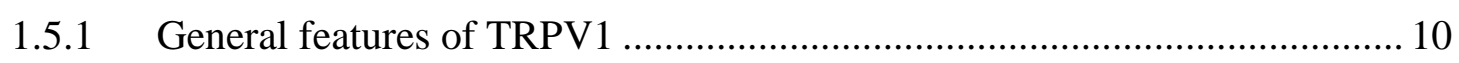

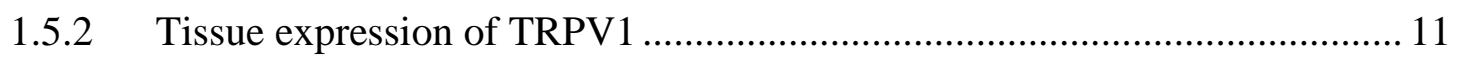

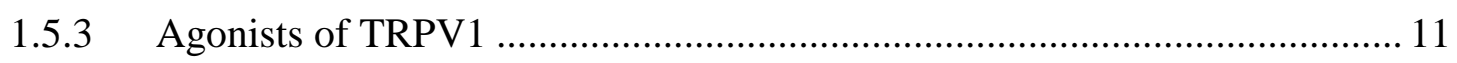

1.5.4 Modulation of TRPV1 function and surface expression ............................... 12

1.5.5 Involvement of TRPV1 in pain conditions................................................ 13

1.6 Targeting protein-protein interactions for pain relief ........................................... 14

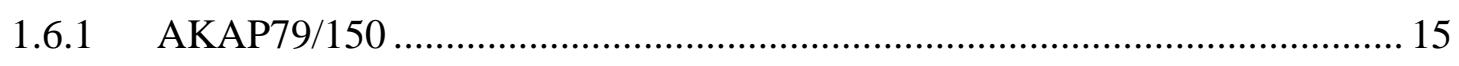

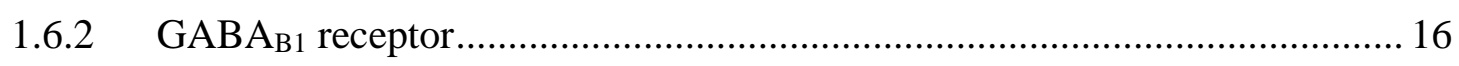

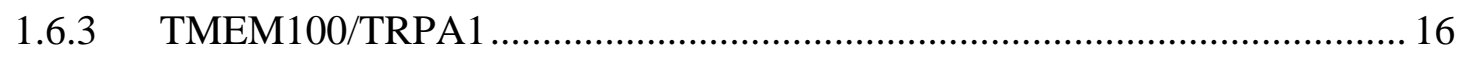

1.7 Mass spectrometry as a tool for the identification of pain-related proteins and

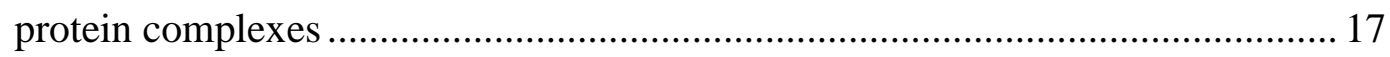

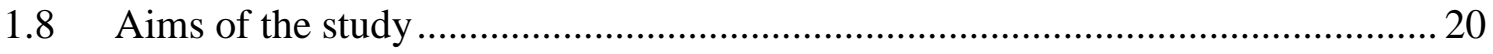

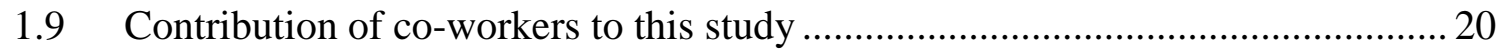

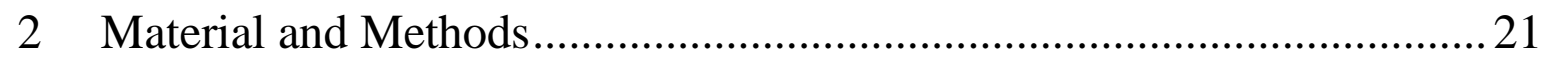

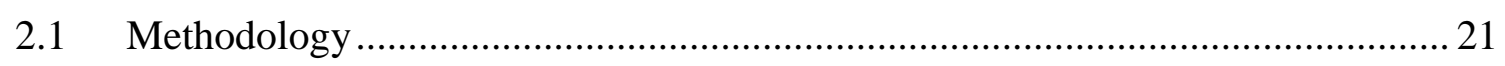

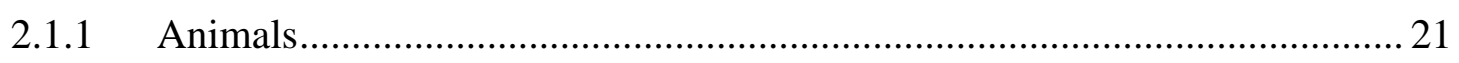

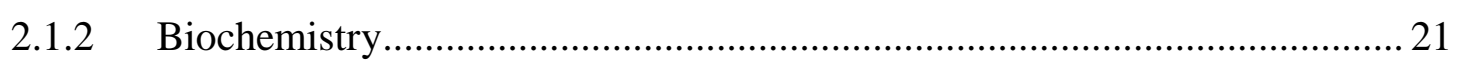




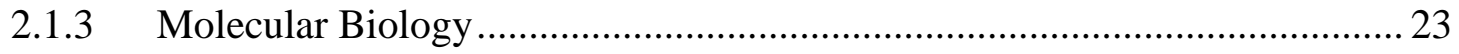

2.1.4 Immunohistochemistry ……………………....................................... 25

2.1.5 Cell culture, cell stainings \& in vitro functional assays ................................ 27

2.1.6 In vivo experiments ......................................................................... 32

2.1.7 Mass spectrometry and bioinformatic analysis ............................................. 35

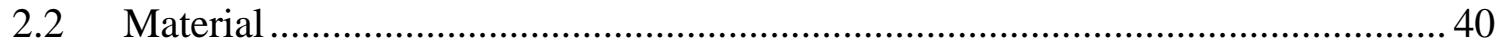

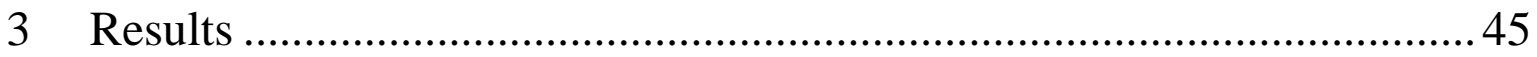

3.1 Insights into chronic pain by standardized DRG proteome profiling ………....... 45

3.1.1 Animal models of chronic pain and sample preparation ............................... 47

3.1.2 Generation of a membrane-enriched spectral library of mouse DRG neurons .

3.1.3 DIA-MS reveals the differential regulation of proteins in rodent models of inflammatory and neuropathic pain....

3.1.4 Alterations of major cellular signalling networks during chronic pain states ...

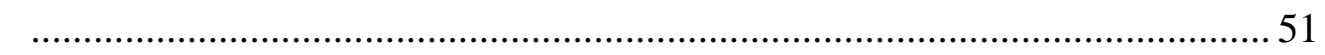

3.2 Identification and characterization of TRPV1 protein complexes..........................55

3.2.1 Validation of Vtilb as modulator of TRPV1-mediated nociceptive signalling .55

3.2.2 In vivo knockdown of Vtilb attenuates thermal hyperalgesia in an

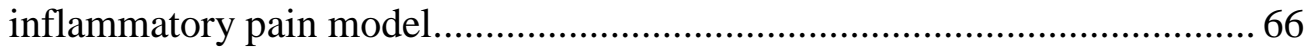

3.2.3 Vtilb belongs to the TRPV1 interactome.......................................................69

3.2.4 Identification of pain-specific interactors of TRPV1 by quantitative mass

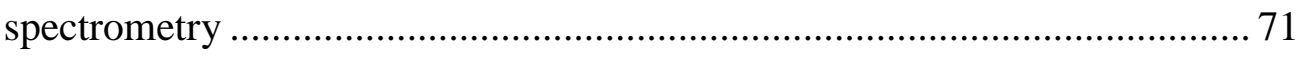

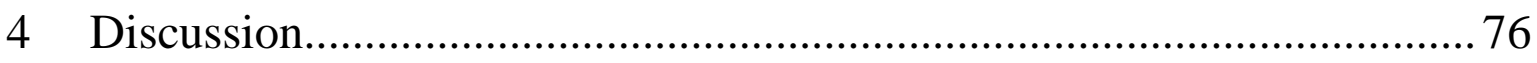

4.1 Insights into chronic pain by standardized DRG proteome profiling ……............ 76

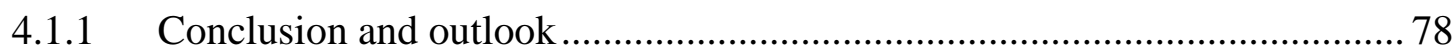

4.2 Identification and characterization of TRPV1 protein complexes........................ 79

4.2.1 Validation of the Vti1b-TRPV1 interaction in vitro...................................... 79

4.2.2 In vivo validation of the Vti1b-TRPV1 interaction....................................... 81

4.2.3 Quantitative MS to identify TRPV1 pain-specific interactors ...................... 82

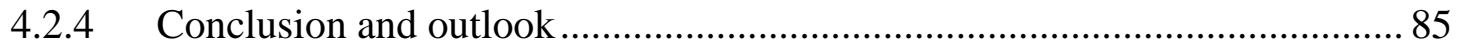

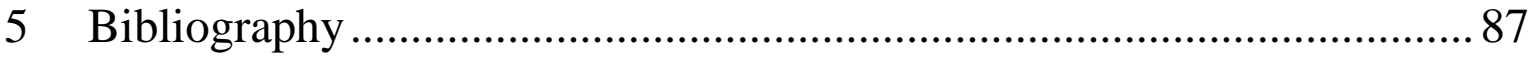

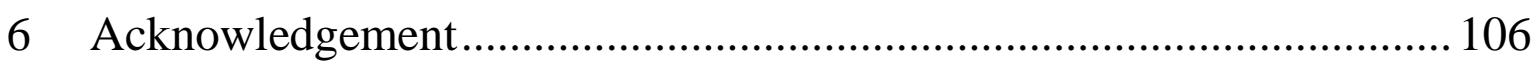

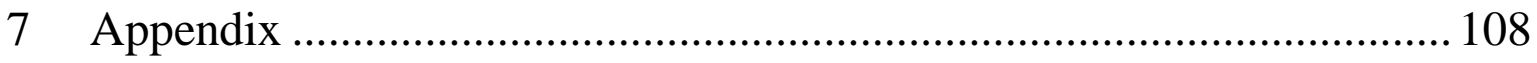

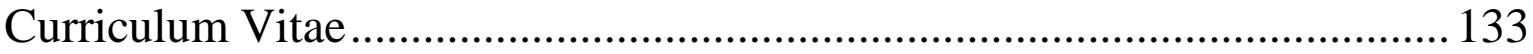




\section{List of Figures}

Figure 1.1: Key stations along the ascending pain pathway ........................................ 3

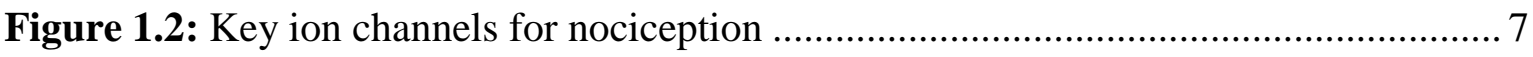

Figure 1.3: Thermosensitive TRP channel in sensory neurons ....................................... 8

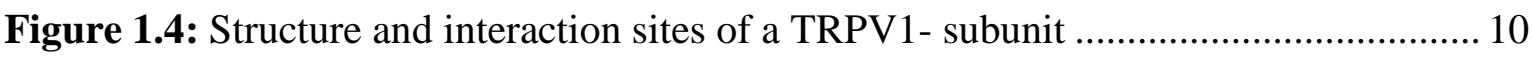

Figure 1.5: Outline of the different steps of a typical proteomics experiment ................... 17

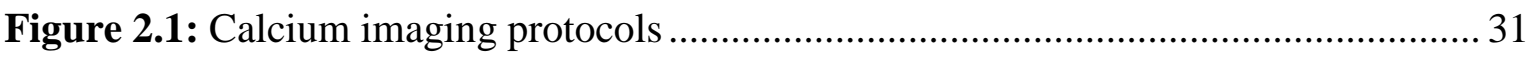

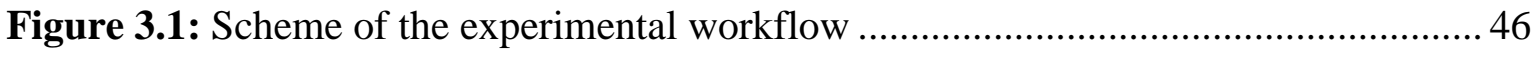

Figure 3.2: Differential regulation of proteins in inflammatory and neuropathic pain ...... 50

Figure 3.3: Role of mitochondrial and PDI activity in chronic inflammatory pain in mice

Figure 3.4: Functional association networks of significantly regulated proteins in the

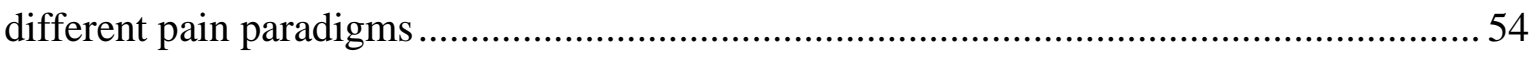

Figure 3.5: Co-expression of TRPV1 and Vtilb in IDRG neurons................................... 56

Figure 3.6: Overexpression of Vtilb alters surface expression, function and desensitization of heterologously expressed TRPV1 channels

Figure 3.7: Vti1b knockdown affects CFA-induced sensitization of TRPV1-expressing sensory neurons

Figure 3.8: Knockdown of Vti1b does not influence TRPA1-mediated neuronal activity 65 Figure 3.9: AAV6-mediated knockdown of Vtilb reduces thermal hyperalgesia after inflammation.

Figure 3.10: Vti1b is in close proximity to TRPV1 both in DRG neurons and upon

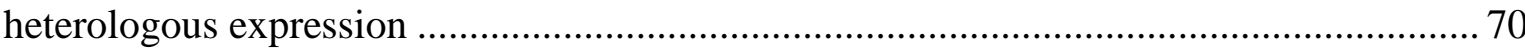

Figure 3.11: Determination of high confidence interacting proteins ............................... 75

Figure 7.1: Additional information about calcium imaging experiments in HEK cells overexpressing TRPV1 + Mock or TRPV1+Vti1b ................................................. 108

Figure 7.2: Additional information on calcium imaging experiments in IDRG neurons . 109 Figure 7.3: Vtilb-KO mice show normal basal sensitivity to mechanical and thermal stimuli and normal capsaicin- and CFA-induced pain behaviour

Figure 7.4: Additional information on sensory behavioural tests in AAV6-Vti1b miRNA mice

Figure 7.5: Western blot of co-immunoprecipitation of TRPV1 and Vti1b in HEK cells 113

Figure 7.6: Correlation of the biological replicates between and within the groups........ 114

Figure 7.7: Distribution of abundance values and imputed values .................................. 115

Figure 7.8: Protein-protein interaction network of Veh-enriched proteins ...................... 131

Figure 7.9: Protein-protein interaction network of CFA-enriched proteins ..................... 132 


\section{List of Tables}

Table 2-1: Details of material used in this study............................ 40

Table 2-2: Details of antibodies across experiments.......................... 42

Table 2-3: Details of DNA plasmids used in this study.......................................... 44

Table 2-4: Details of siRNA used in this study..................................................... 44

Table 2-5: Details of qPCR-primers used in this study......................................... 44

Table 7-1: Pain-specific interactors of TRPV1 ................................................. 116

Table 7-2: Putative TRPV1 interactors not detected in the KO group..................... 122

Table 7-3: Enriched annotation term clusters for Veh-enriched proteins and proteins only detected in Veh......................................................... 126

Table 7-4: Enriched annotation term clusters for CFA-enriched proteins and proteins only detected in CFA. 


\section{List of Abbreviations}

\begin{tabular}{|c|c|}
\hline BDNF & Brain-derived neurotrophic factor \\
\hline $\mathrm{Bk}$ & Bradykinin \\
\hline $\mathrm{CaM}$ & Calmodulin \\
\hline Caps & Capsaicin \\
\hline CFA & Complete Freund's Adjuvant \\
\hline CNS & Central nervous system \\
\hline CoIP & Co-immunoprecipitation \\
\hline DDA & Data-dependent acquisition \\
\hline DIA-MS & Data-independent acquisition mass spectrometry \\
\hline DRG & Dorsal root ganglion/ganglia \\
\hline DTT & Dithiothreitol \\
\hline $\mathrm{EtOH}$ & Ethanol \\
\hline FDR & False discovery rate \\
\hline Fig. & Figure \\
\hline GAPDH & Glyceraldehyde-3-phosphate dehydrogenase \\
\hline GDNF & Glial cell line-derived neurotrophic factor \\
\hline GFP & Green fluorescent protein \\
\hline GO & Gene ontology \\
\hline GPCR & G protein-coupled receptor \\
\hline $\mathrm{h}$ & Hour(s) \\
\hline HIV & Human immunodeficiency virus \\
\hline i.p. & Intraperitoneal \\
\hline i.a.u., AU & In arbitary units \\
\hline IL & Interleukin \\
\hline IMS & Ion-mobility separation \\
\hline $\mathrm{KO}$ & Knockout \\
\hline LC-MS & Liquid chromatography-MS \\
\hline LDRG & Lumbar DRG \\
\hline $\min$ & Minutes \\
\hline miRNA, miR & MicroRNA \\
\hline $\mathrm{MO}$ & Mustard oil \\
\hline MS & Mass spectrometry \\
\hline MS/MS & Tandem MS \\
\hline na & Not applicable \\
\hline $\mathrm{NaN}$ & Not a number \\
\hline NGF & Nerve growth factor \\
\hline NMDA & N-Methyl-D-Aspartate \\
\hline ns & Not significant \\
\hline NT-3 & Neurotrophin-3 \\
\hline NT-4 & Neurotrophin-4 \\
\hline PBS & Phosphate-buffered saline \\
\hline
\end{tabular}




$\begin{array}{ll}\text { PCR } & \text { Polymerase chain reaction } \\ \text { PGE2 } & \text { Prostagladin E2 } \\ \text { PKA } & \text { Protein kinase A } \\ \text { PKC } & \text { Protein kinase C } \\ \text { PLA } & \text { Proximity ligation assay } \\ \text { PLC } & \text { Phospholipase C } \\ \text { PNS } & \text { Peripheral nervous system } \\ \text { qPCR } & \text { Quantitative PCR } \\ \text { RNAi } & \text { RNA interference } \\ \text { RT } & \text { Room temperature } \\ \text { S } & \text { Second(s) } \\ \text { s.c. } & \text { Subcutaneous } \\ \text { siRNA } & \text { Small interfering RNA } \\ \text { SNARE } & \text { Soluble N-ethylmaleimide-sensitive- } \\ \text { SWATH } & \text { factor attachment receptor } \\ & \text { Sequential window acquisition of all theoretical } \\ \text { TNF } \alpha & \text { mass spectra } \\ \text { TrkA } & \text { Tumor necrosis factor } \alpha \\ \text { TRP } & \text { Tropomyosin receptor kinase A } \\ \text { TRPA1 } & \text { Transient receptor potential } \\ \text { TRPV1 } & \text { TRP channel, subfamily Ankyrin, member 1 } \\ \text { UDMS } & \text { TRP channel, subfamily Vanilloid, member 1 } \\ \text { UPLC } & \text { Ultra-definition MS } \\ \text { Veh } & \text { Ultra-performance LC } \\ \text { Vtilb } & \text { Vehicle } \\ \text { WT } & \text { Vesicle transport through interaction with t- } \\ \text { YFP } & \text { SNAREs homolog 1B } \\ & \text { Wild type } \\ \text { Yellow fluorescent protein } \\ \\ \text { PNA }\end{array}$




\section{Abstract}

Chronic pain imposes substantial challenges to medical practice as the treatment options for its clinically relevant manifestations are limited. Owing to lack of knowledge about the exact molecular mechanism underlying pathological pain conditions, pain therapeutics currently available target molecules with key physiological functions in our body. Thus, they are accompanied by severe side effects, which limits effective dosage prescription. Bearing these difficulties in mind, it is highly desirable to identify the proteins and their associated complexes that are differentially regulated and function at the forefront of noxious stimulus detection. The goal of this study was to identify and characterize multiprotein complexes in the context of nociception in mice. On the one hand, I followed a unbiased mass spectrometry (MS)-based approach to characterize the changes in expression of a large set of proteins in the dorsal root ganglia (DRG). DRG harbour the somata of nociceptors, the primary afferent sensory neurons that express distinct molecular sensor of painful stimuli. On the other hand, I aimed to reveal the scaffold of proteins interacting with the Transient Receptor Potential V1 (TRPV1) ion channel, a polymodal sensor of irritant chemicals and noxious heat.

In order to elucidate the molecular underpinnings of chronic pain, several large-scale profiling studies have been performed. However, the generated lists of regulated candidate proteins are often inconsistent with scarce overlap. This could be explained by inherent technical limitations of used proteomics methods, such as low reproducibility. Emerging data-independent acquisition mass spectrometry (DIA-MS) has the potential to allow for standardized and reproducible quantification across many samples. Here, we applied DIAMS on DRG isolated from mice subjected to two mouse models of chronic pain to define global changes in the DRG proteome. More specifically, we compared the abundance of 2,526 DRG proteins across the two pain models and their respective controls. Considerable and pain-model specific alterations in the abundance of several dozen proteins as well as within functional protein networks were detected. These were validated with several orthogonal methods. Amongst others, the analysis of mouse pain behaviours verified that meaningful protein alterations both on the level of single proteins and signalling networks were revealed with our workflow.

The involvement of TRPV1 in different chronic pain states has been well documented and together with its enriched expression in DRG renders TRPV1 a promising target for novel analgesics. However, several TRPV1 antagonists that reached clinical trials are challenged by severe side effects because of interference with physiological functions of TRPV1. An interesting alternative to TRPV1 blockage might be the targeting of such TRPV1 interaction partners that are specific for e.g. inflammatory pain. This strategy would provide a means to suppress pathological pain states whilst leaving nociceptive pain intact. However, very little is known about the protein scaffold of TRPV1 during different pain states. Here, I present the Vesicle transport through interaction with t-SNAREs homolog 1B (Vtilb) as a novel pain-specific interactor of TRPV1. Vtilb modulates TRPV1 sensitization within an 
inflammatory milieu in vitro. Normal functioning of the TRPV1 is left intact. In vivo virusmediated knockdown of Vtil b diminished the development of thermal hypersensitivity upon CFA injection in mice. The knockdown does not affect CFA-evoked mechanical hypersensitivity or capsaicin-induced nocifensive behaviour. In a second step, a functional proteomics approach was employed to identify the TRPV1 interactome under CFA-induced inflammatory pain in mouse DRG neurons. Comparison of the interactomics data between the control and CFA group revealed a significant regulation of the TRPV1 interactome upon induction of inflammatory pain. For instance, Vti1b was found to be less abundant in TRPV1 protein complexes upon inflammation. Overall, this study strongly supports the notion that protein-protein interactions specific for pathological pain exist.

In summary, these two mass spectrometric studies represent a unique resource on (I) the differential expression of membrane proteins during pathological pain and (II) the dynamics of TRPV1 interactors during inflammatory pain. Acquired data may contribute to the characterization of the molecular mechanisms underlying pathological pain and may therefore facilitate the development of more effective therapeutic strategies. 


\section{Introduction}

\subsection{Pain}

Pain is defined as "an unpleasant sensory and emotional experience associated with actual or potential tissue damage, or described in terms of such damage" by the International Association for the Study of Pain (Merskey and Bogduk, 1994). It acts as a sensor for potentially harmful environmental stimuli. It informs about internal organ dysfunction, and is therefore crucial for the physical integrity of our body and, thus, our survival. Humans with rare congenital insensitivity to pain suffer from recurrent injuries and self-mutilation often followed by severe infections, since no protective behaviour is triggered (Cox et al., 2006; Indo et al., 1996; Leipold et al., 2013).

On the other hand, pain is the number one reason worldwide for seeking medical attention. If the pain persists long after the initial cause has been eliminated, and becomes chronic, it loses its evolutionary, protective aspect, and becomes a disease in its own right. Chronic pain is a highly debilitating condition and can profoundly affect quality of life. It is often associated with comorbidities such as depression and anxiety. The resulting loss of productivity together with the high cost of treatment turn chronic pain into a burden for society as a whole (Breivik et al., 2013; Dueñas et al., 2016). Chronic pain affects nearly $20 \%$ of the adult European population and imposes substantial challenges to patients and physicians alike, as current options for pain treatment are limited (Breivik et al., 2006). A Europe-wide survey reported that pain management is inadequate in about $40 \%$ of affected people (Breivik et al., 2006).

Currently available treatment options include nonsteroidal anti-inflammatory drugs, anticonvulsants, antidepressants and opioids (Turk et al., 2011). Yet, treatment remains a challenge, as the majority cause adverse side effects such as constipation, nausea and sedation, thus limiting their effectiveness (Kroenke et al., 2009). The main reason is that the exact molecular mechanism underlying different chronic pain syndromes are not known. Medication targets molecules ubiquitously expressed in the body and interferes with their key physiological functions. Thus, major efforts are being made to discover the molecular underpinnings of chronic pathological pain to allow for causative treatment.

\subsection{Nociception and pain - from the detection of noxious stimuli in the periphery to the sensation of pain}

Nociceptive pain is categorized as a submodality of somatosensation, which also comprises the important senses of proprioception, touch and thermosensation.

The first step in the complex neural process that ultimately leaves us with the very subjective and emotional sensation we call pain, involves the activation of nociceptors. Nociceptors are a specialized subtype of primary afferent sensory neurons with the ability to encode external 
noxious stimuli into electrical signals. They innervate the skin, blood vessels and internal organs, and are equipped with distinct receptor molecules which transduce potentially harmful stimuli into electrical signals. These are then transmitted via several relay stations to higher brain centres. The brain not only creates a conscious percept of pain but also initiates the appropriate protective behavioural and emotional responses. (Basbaum and Jessell, 2013)

Three main classes of nociceptors can be distinguished dependent on the set of ion channels and receptors expressed: (I) thermal - excited by temperature extremes (for humans $<\sim 15^{\circ} \mathrm{C}$ and $>\sim 40-45^{\circ} \mathrm{C}$ ), (ii) mechanical - activated by intense mechanical pressure and (iii) polymodal - excited by both mechanical, thermal and chemical noxious stimuli (Dubin and Patapoutian, 2010). There is also a fourth, rather enigmatic class, the "silent" nociceptors that start to respond to noxious thermal and mechanical stimulation only after sensitization by inflammatory processes accompanying tissue injury (Gold and Gebhart, 2010; Schmidt et al., 1995). Nociceptors can also be distinguished due to differences in the diameter of their fibres correlating with the conduction speed of action potentials. The so-called C-fibres, which comprise the major part of nociceptors, have thin, unmyelinated axons (diameter of 0.2-1.5 $\mu \mathrm{m}$ ) allowing conduction speeds of no more than 0.4-1.4 m/s. A $\delta$-fibres, on the other hand, are on average 2 to $5 \mu \mathrm{m}$ in diameter, thinly myelinated with conduction velocities of 5-30 m/s. Hitting our toes for example on a table-leg, we feel an initial, fast onset pain, usually sharp and well localized that has been linked to the A $\delta$-fibres. The "second" slowonset pain, conveyed by C-fibres is described as dull, throbbing and diffusely located. In addition, the expression of certain neuro-peptides (see below), or transmembrane proteins as well as responsiveness to distinct growth factor are used as markers to classify this highly heterogeneous population of neurons.(Basbaum and Jessell, 2013)

Nociceptors contain three main parts, a soma, a peripheral and a central process. The somata of nociceptive neurons are organized in peripheral sensory ganglia. Of these, the dorsal root ganglia (DRG) are lined up along the spinal cord (Fig. 1.1). Their neurons are mainly responsible for limb and trunk innervation; facial innervation is ensured by the trigeminal ganglia (TG). A subset of vagal afferent sensory neurons innervating the lung and oesophagus that are suggested to be nociceptors have their somata in the sympathetic nodose and jugular ganglia (Basbaum and Jessell, 2013; Kollarik et al., 2010).

Nociceptors possess a pseudo-unipolar morphology, i.e. from their soma a single, short process emanates and bifurcates into a peripheral and central process. Near to the target organ the peripheral process branches out and terminates into free nerve endings. Terminals of the central axon synapse on second-order neurons in the dorsal horn (DH) of the spinal cord (SC; Fig. 1.1). 


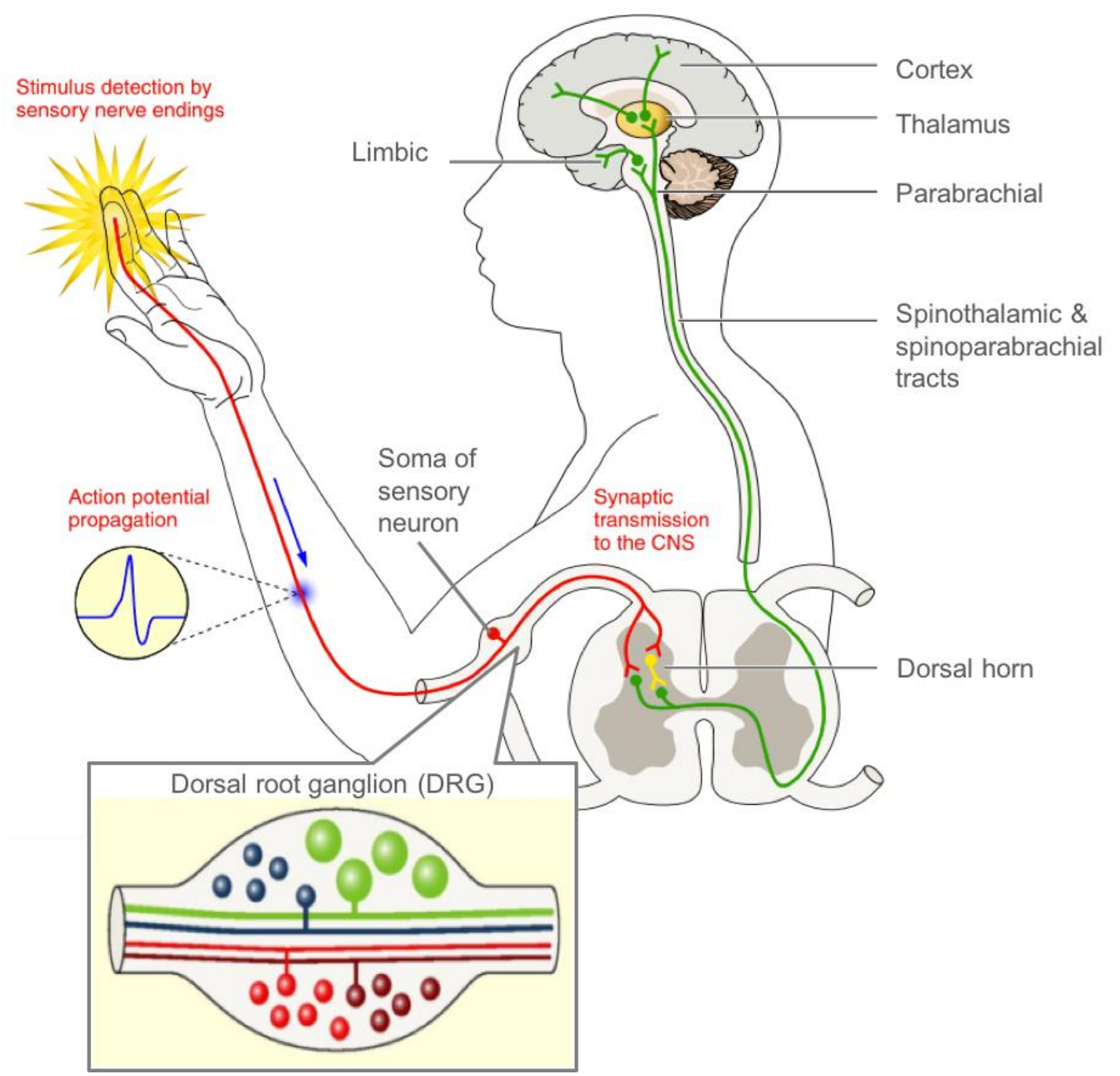

Figure 1.1: Key stations along the ascending pain pathway

Primary afferent sensory neurons detect noxious and innocuous chemical and physical stimuli in the periphery and encode them into electrical signals that are conveyed via several relay stations to the brain. The dorsal root ganglion (DRG) houses the somata of these pseudo-unipolar neurons that possess a peripherally and a centrally projecting process. Within the DRG several subtypes of primary afferent neurons can be distinguished: (I) small- to medium-diameter cells giving rise to Cfibres and $\mathrm{A} \delta$-fibres, respectively: mainly nociception and thermosensation; (II) large-diameter neurons with $A \alpha / \beta$-fibres: mainly touch and proprioception. The sensory information can be processed in local neuronal circuits in the first relay station, the dorsal horn of the spinal cord, before it is sent via the thalamus to higher brain areas where the percept of pain is finally created. Adapted from Bourinet et al., 2014.

At the central synapse, the major excitatory neurotransmitter is glutamate; a subgroup of nociceptors also releases several neuro-peptides as co-transmitters (substance P (SP), calcitonin gene-related peptide (CGRP), somatostatin and galatin). The modulatory action of the co-transmitters on the firing properties of the DH neurons underlie synaptic plasticity, a basis for the sensitized processing of nociceptive input occurring under pathological pain conditions. (Basbaum et al., 2009)

The afferent fibres project to distinct laminae of the unilateral dorsal horn; the C-fibres terminate on laminae I and II, whilst A $\delta$-fibres synapse on neurons in both the laminae I and $\mathrm{V}$. Both projection neurons as well as excitatory and inhibitory local interneurons are 
targeted by the presynaptic terminals of the nociceptors. Fibres of the projection neurons give rise to five major ascending tracts in the antero-lateral system to convey the nociceptive information to supraspinal structures:

I. Spinothalamic,

II. spinoreticular,

III. spinomesencephalic/parabrachial,

IV. cervicothalamic and

V. spinohypothalamic tract.

The most important one is the spinothalamic tract carrying multi-modal sensory information to both lateral and medial nuclei of the thalamus. The sole electrical stimulation of this tract elicits the perception of pain. From the thalamus the nociceptive information gets distributed to different cortical areas. There is not one specific pain centre, but several structures processing multimodal information are targeted (Apkarian et al., 2005). For the sensory discriminative component of pain, the somatosensory cortex is responsible. Several other structures such as the insular cortex (limbic structures) and cingulate circuit are believed to mediate the emotional and aversive component of pain perception. Also, the fibres of the other minor tracts are thought to contribute to the affective component of pain. The spinomesencephalic tract terminates on the mesencephalic reticular formation as well as the periaqueductal gray (PAG) in the brainstem and parabrachial nuclei from where neurons project to the amygdala. Information carried along the spinohypothalamic tract is integrated in the hypothalamic nuclei eliciting neuroendocrine and cardiovascular responses. (Basbaum and Jessell, 2013)

The percept of pain can be greatly influenced by the emotional state, past experiences, and attention. The most remarkable example is that of soldiers being severely wounded in combat but only report pain once they have left the battlefield (Beecher, 1946). Descending pathways arising from the brainstem (RVM and PAG) and higher brain areas (cingulofrontal regions, the amygdalae and the hypothalamus) can significantly modulate the processing of nociceptive input. Modulation can be either inhibitory or facilitative, and the transmitters involved are serotonin, monoamines and endogenous opioids. There is no anatomical distinction between paths that potentiate or inhibit the response of presynaptic and postsynaptic spinal pain transmission neurons. Even the same transmitter can have dual effects depending on the subtype of receptors it binds or the functionally distinct neurons it targets. (Millan, 2002)

\subsection{Sensitization of the nociceptor - paving the way for pathological pain}

The intricate interplay of the different structures along the pain pathway allows for a reasonable interpretation of the initial nociceptive insult in the context of previous experience and current bodily state which should result in the most appropriate action for survival. Yet, due to ongoing inflammation, injury and disease, pain can become maladaptive and remain long after the initial cause has disappeared. Pain and the 
accompanying bodily reactions not only lose their usefulness in the sense of serving any protective function but also lead to great suffering for the patient. Despite intense research, the underlying pathophysiological changes underlying manifold chronic pain syndromes are not fully understood leaving poor treatment options. (Vardeh et al., 2016)

Under physiological conditions, the nociceptor is tuned to respond only to high threshold stimuli that signal an impeding or actual threat to body integrity, and trigger the signal transmission cascade finally initiating withdrawal or any other appropriate reactions to avoid (further) damage. In case of tissue damage, the subsequent inflammation in the tissue causes the nociceptors to transiently adapt a heightened responsiveness. This is to limit further damage, aid wound healing and repair (inflammatory pain, e.g. heightened skin sensitivity to touch due to sunburn). Once the underlying cause or disease has healed, the hypersensitivity state and/or pain should subside. Yet, for mostly unknown reasons it becomes persistent in some cases. Thus, eventually, a disease manifests itself. (Costigan et al., 2009)

This persistent pain manifests as

I. hyperalgesia - exaggerated response to a noxious stimulus,

II. allodynia - normally innocuous stimuli elicit pain and

III. spontaneous pain - pain percept in the absent of any identifiable peripheral stimulus.

These symptoms underlie a variety of clinical pain syndromes that can have different aetiologies. Apart from persistent inflammatory pain, we define neuropathic pain - any pain caused by injury or disease to the somatosensory system, and dysfunctional pain amplification of nociceptive signalling in the absence of either inflammation or neural lesions. Whilst some neuropathic pain conditions are initiated and maintained at the CNS (such as pain resulting from thalamic stroke or spinal cord injury), many have started with an insult of peripheral nerve fibres. This can be mechanical injury, ongoing metabolic diseases (e.g. painful diabetic neuropathy), action of neurotoxic substances (e.g. chemotherapy-induced neuropathic pain), due to infections (e.g. post-herpetic neuralgia and HIV-associated neuropathy) and invasion of tumours. (Costigan et al., 2009)

\subsubsection{Peripheral sensitization - functional changes in nociceptors}

If there is tissue damage, the activated nociceptors, injured cells and resident and attracted immune cells release inflammatory mediators into the injured area. Several amines, kinins, growth factors, prostaglandins, chemokines, as well as ATP and protons constitute what is called the "inflammatory soup" and act in-/directly on nociceptors to sensitize them (Woolf and $\mathrm{Ma}, 2007)$. Some of these molecules, for instance, the cytokines tumour necrosis factor $\alpha$ (TNF $\alpha$ ), interleukin-1 $\beta$ (IL-1 $\beta$ ) and IL-6 (Ritner et al., 2009), seem to be mainly responsible for potentiating the inflammatory response itself. Others act via their respective receptor (mostly ligand-gated ion channels and G protein-coupled receptors (GPCR)) that are expressed by the nociceptors. They thereby activate intracellular signalling cascades with downstream mediators such as protein kinase C (PKC), PKA, phosphoinositide 3-kinase (PI3K) and MAP kinases ERK and p38. The targets of these kinases are amongst others the 
ion channels that are essential for the transduction process at the nociceptive terminals as well as the ones generating action potentials and conveying central transmission. Their phosphorylation results mainly in the potentiation of their action either due to alteration in ion channel kinetics or in the increased trafficking of functional channels to the plasma membrane, thereby lowering the threshold of activation such that also innocuous stimuli excite nociceptors. Furthermore, previously silent nociceptors are activated by the inflammatory mediators and start to respond to stimuli. This increased processing of external stimuli manifest as hyperalgesia and allodynia. (Basbaum et al., 2009; Woolf and Ma, 2007)

Chronic pain patients often report spontaneous pain. Spontaneous pain is thought to result from aberrant ectopic discharges in absence of a stimulus in the peripheral terminals (von Hehn et al., 2012). Injuries to the axonal part of the nociceptor trigger changes in expression, trafficking and clustering of certain types of voltage-gated sodium channels important for the generation and conduction of action potentials. This causes unstable oscillations of the membrane potential which gives rise to abnormal firing (Amir et al., 2002). Ectopic activity is not only generated in the injured fibres but also in the adjacent uninjured ones ( $\mathrm{Wu}$ et al., 2002).

Heightened sensitivity and persistent activity of the peripheral nociceptor inevitably affects the second-order sensory neurons in the DH that undergo several phenotypic changes. Amongst others, alterations occur in the glutamatergic neurotransmission leading to increase in synaptic strength. This activity-dependent neuroplasticity further enhances processing of nociceptive signals (central sensitization; (Kuner, 2010)).

\subsection{TRP ion channels - polymodal signal integrators}

Every step of nociception - from the initial transduction of noxious stimuli to the generation and propagation of action potentials and the synaptic transmission in the SC - is mediated by a group of transmembrane proteins, namely ion channels (Waxman and Zamponi, 2014). Fig. 1.2 summarizes the key ion channels in the primary afferent nociceptive neuron. Collectively, they shape the activation characteristics of nociceptors. Any dysregulation in their expression due to injury or disease can cause an enhancement of neuronal excitability underlying chronic neuropathic and inflammatory pain (Delmas, 2008). 


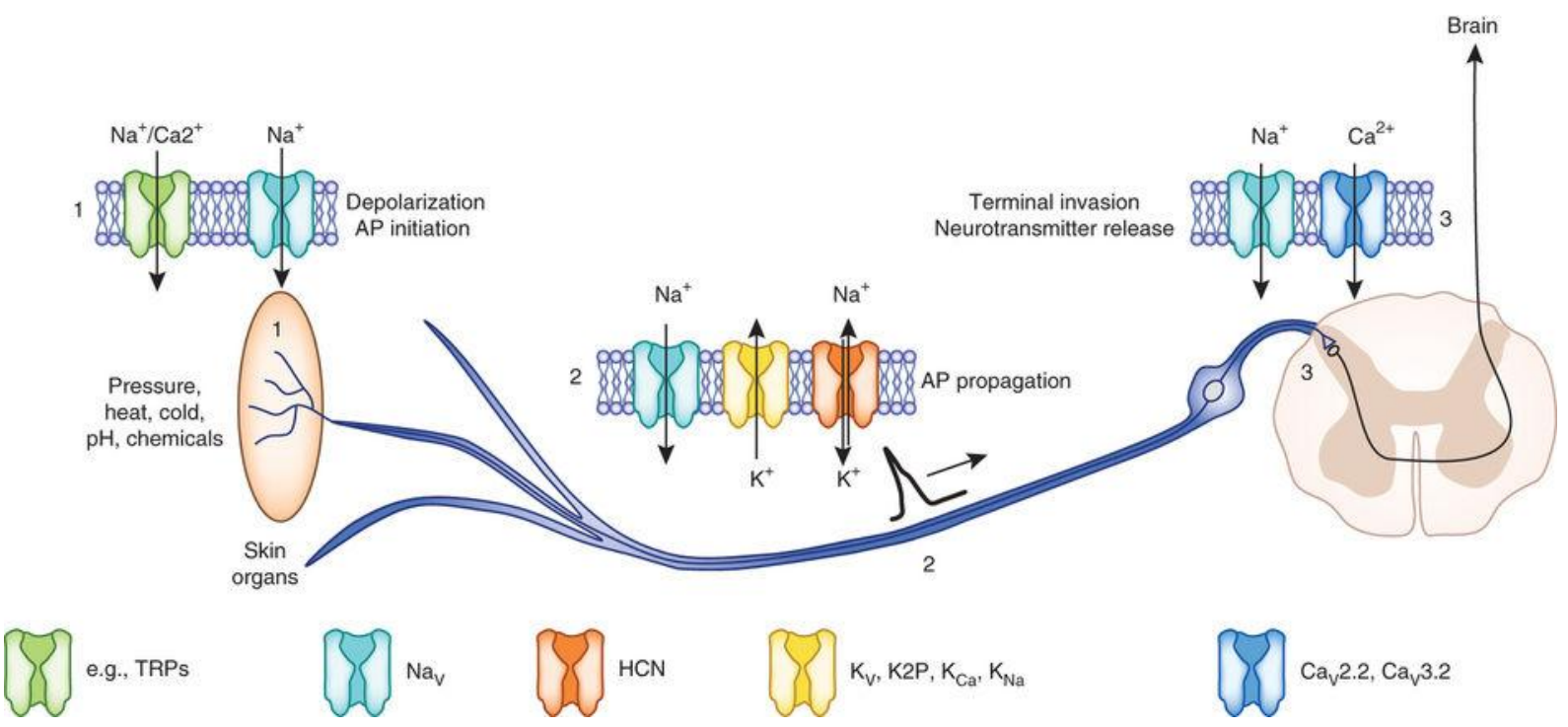

Figure 1.2: Key ion channels for nociception

Located at the peripheral terminals (1) of primary afferent neurons, e.g. TRP ion channels transduce noxious stimuli of different modality into membrane depolarisations. Action potentials are initiated (1) and propagated (2) along the afferent fibres via activity of voltage-gated potassium and sodium channels. At the central terminals (3), voltage-gated calcium channels are activated by the incoming action potentials and cause neurotransmitter release at the synapse. Below the scheme, examples of the involved ion channel subgroups are mentioned. $\mathrm{Ca}_{v}$, voltage-gated calcium channel; $\mathrm{HCN}$, hyperpolarization-activated cyclic nucleotide-gated channel; K2P, two-pore potassium channel; $\mathrm{K}_{\mathrm{Ca}}$, calcium-activated potassium channel; $\mathrm{K}_{\mathrm{Na}}$, sodium-activated potassium channel; $\mathrm{K}_{\mathrm{V}}$, voltage-gated potassium channel; Nav, voltage-activated sodium channel; TRP, transient receptor potential ion channel. Reprinted by permission from Springer Nature: Nature Neuroscience, Regulating excitability of peripheral afferents: emerging ion channel targets. Waxman and Zamponi, 2014.

In the last decades, members of the Transient Receptor Potential (TRP) superfamily of ion channels have been discovered for the detection and transduction of a wide range of noxious or innocuous chemical and physical stimuli. Their name "transient receptor potential", refers to the phenotype observed in the electroretinogram from mutant Drosophila melanogaster that, when exposed to intense prolonged light stimulus, exhibited a transient instead of the rather sustained wild type response (Minke, 2010). TRP channels are key players in a diversity of sensory systems, for instance, vision, thermosensation and osmoregulation. They allow animals to sense changes in the environment and therefore critically guide their behaviour. Not surprisingly, TRP-related channels are found to be conserved amongst invertebrates and vertebrates (Nilius and Flockerzi, 2014).

So far 28 TRP genes have been found in mammals. Generally, they are non-selective cation permeable channels, with many of them showing a relatively high permeability for calcium. Their family togetherness is based mainly on sequence homology as both selectivity and activation modus varies a lot. They share a common structure of 6 transmembrane spanning domains with intracellular located $\mathrm{N}$ - and $\mathrm{C}$ - terminals; formation of tetramers is required for a functional channel. The cytosolic domains contain many sites for regulation by protein kinases, chaperones, and scaffolding proteins. Based on the degree of homology in their amino acid sequence the following subgroups have been defined: TRPC (Canonical, 
TRPC1-7), TRPA (Ankyrin, only TRPA1), TRPM (Melastatin, TRPM1-8), TRPML (Mucolipin, TRPML1-3), TRPP (Polycystin, TRPP1-3), and TRPV (Vanilloid, TRPV1-6). (Nilius and Owsianik, 2011)

It is remarkable with TRP channels that one single channel can be activated by a surprisingly broad range of both external and internal sensory stimuli which endows them to act as polymodal signal integrators of changes in the tissue and cellular environment (Clapham, 2003). Given this important role as "cellular sensors" (Clapham, 2003) and their expression in several cell types in nearly every tissue, it is not surprising that mutations in TRP genes underlie a diversity of human diseases affecting the cardiovascular, renal, skeletal and nervous system (Kaneko and Szallasi, 2014).

\subsubsection{TRP channels - transducer of nociceptive stimuli}

Some members of the superfamily of TRP channels are tuned to detect noxious stimuli and are involved in pathological pain states. Among these are TRPV1-4, TRPM2, $3 \& 8$, TRPA1 (Sousa-Valente et al., 2014) as well as TRPC1 \& TRPC6 (Alessandri-Haber et al., 2009). Apart from TRPC1 \& TRPC 6, they belong to the "thermoTRPs", a subgroup of TRP channels that display an increase in open probability of the channel to a certain temperature range (Fig. 1.3).

\section{Thermosensing nociceptive TRP channels in sensory neurons}

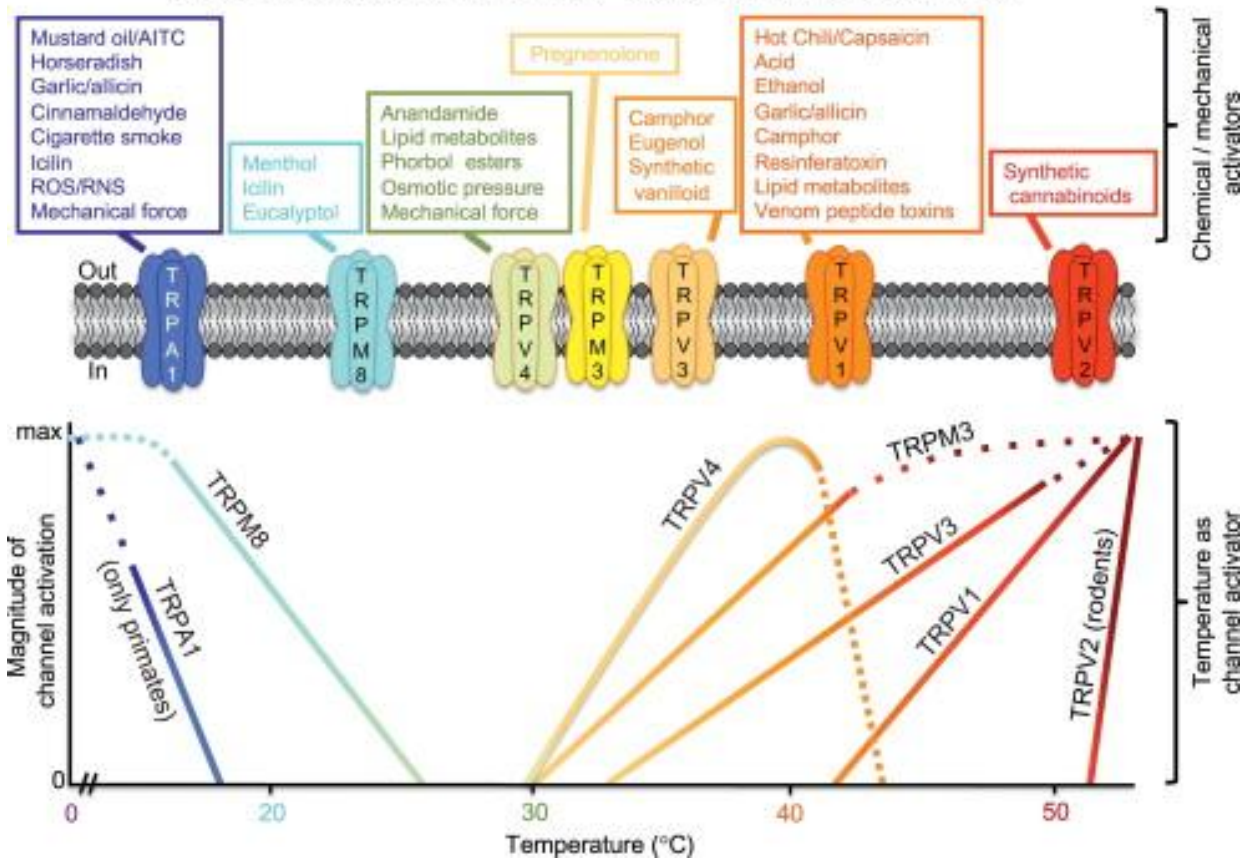

Figure 1.3: Thermosensitive TRP channel in sensory neurons

ThermoTRPs comprise a subgroup of TRP channels that are activated by temperatures ranging from noxious cold to noxious heat. Non-thermal activators of each thermoTRPs are displayed at the top with many of them being known to induce thermal and pain sensations in humans. The distinct activation thresholds and maximal activation of these channels measured in heterologous expression systems are depicted below. Uncertainty of the slopes are indicated with dashed lines. Reprinted from Progress in Molecular Biology and Translational Science, 131, Mickle, Shepherd and Mohapatra, Sensory TRP channels: The key transducers of nociception and pain, 73-118, 2015, with permission from Elsevier. 
Acting in the first line of stimulus detection and being sensitized under pathological pain conditions, render them - some more than others - prime targets for the development of novel analgesics. So far TRPV1 and TRPA1 - both showing an enriched expression in neurons of peripheral ganglia - have attracted the most attention with respect to their role in pathological pain states (Sousa-Valente et al., 2014).

TRPA1 is a key player in different types of pain (Nassini et al., 2014). The channel shows an enriched expression in nociceptive neurons and in DRG a high co-expression with TRPV1 (Story et al., 2003). TRPA1 is activated by a wide range of heterogonous compounds that induce acute painful burning sensation like pungent natural compounds, e.g., mustard oil (MO), or environmental irritants. A variety of inflammatory mediators and by-products of oxidative and nitrative stress can either directly activate TRPA1 or sensitize the channel contributing to pain hypersensitivity in several animal models of pathological pain (Nassini et al., 2014). Trpal-deficient mice display profound deficiencies in pain response to MO and formalin as well as exhibit attenuated mechanical allodynia upon inflammation (Bautista et al., 2006; da Costa et al., 2010; Kwan et al., 2006; McNamara et al., 2007). Also, in several rodent models of neuropathic pain, the genetic ablation and pharmacological inhibition of TRPA1 results in diminished cold and mechanical hyperalgesia, making it a prime target for the development of novel analgesics (Nassini et al., 2014). Moreover, a gain-of-function mutation in human TRPA1 is causative of Familial Episodic Pain Syndrome (FEPS). Individuals with FEPS suffer from episodic pain attacks mainly localized in the upper body and triggered by fatigue and fasting (Kremeyer et al., 2010).

TRPV1, a sensor of noxious heat and a variety of irritable endo- and exogenous molecules as well as the focus of project II of this thesis, is going to be discussed in more detail below.

\subsection{TRPV1 - the capsaicin receptor}

The molecular identity of the "capsaicin receptor" was revealed in 1997 by expression cloning of the respective gene from a rodent sensory neuron cDNA library that was followed by measurement of calcium influx upon capsaicin and heat stimulation (Caterina et al., 1997). Early on, TRPV1 caught the interest of pharmaceutical companies as a target for novel analgesics. Both the fact that inhibition of TRPV1 was shown to have therapeutic value in several animal pain models and its enriched expression in the DRG render TRPV1 a promising target for the development of effective new analgesics with minimal adverse side effects. Yet, 20 years after its initial cloning, no TRPV1 antagonist has been approved by the FDA (U.S. Food \& Drug Administration). The emergence of adverse side effects in (pre-)clinical trials of $1^{\text {st }}$ generation TRPV1 antagonist (Gavva et al., 2008; Patapoutian et al., 2009; Rowbotham et al., 2011) impeded the initial enthusiasm for inhibiting TRPV1 for pain relief. Some antagonists evoked hyperthermia, confirming the debated role of TRPV1 in the maintenance of body temperature (Gavva et al., 2007). Others interfered with the function of TRPV1 as a sensor for noxious heat - reported elevated heat thresholds in study participants could lead to accidental burn injuries (Carnevale and Rohacs, 2016). A better strategy therefore would be to inhibit only the pathologically active TRPV1 (FernándezCarvajal et al., 2012). 


\subsubsection{General features of TRPV1}

TRPV1, the founding member of the vanilloid subgroup of TRP channels, exhibits the same basic modular architecture as other TRP group members, i.e. 6 transmembrane domains (S1S6) with S5 and S6 being linked by a hydrophilic pore loop (Fig. 1.4). Both N- and C- termini are cytosolic. Four of the transmembrane modules assemble to form a functional channel with the S5-linker-S6 determining the ion pore as shown by high-resolution cryo-electron microscopy studies (Cao et al., 2013; Liao et al., 2013). Opening of the ion pore leads to an influx of mono- and bivalent cations (permeability $\mathrm{P}_{\mathrm{Ca} 2+} / \mathrm{P}_{\mathrm{Na}+} \sim 5$ ) (Nilius and Flockerzi, 2014).

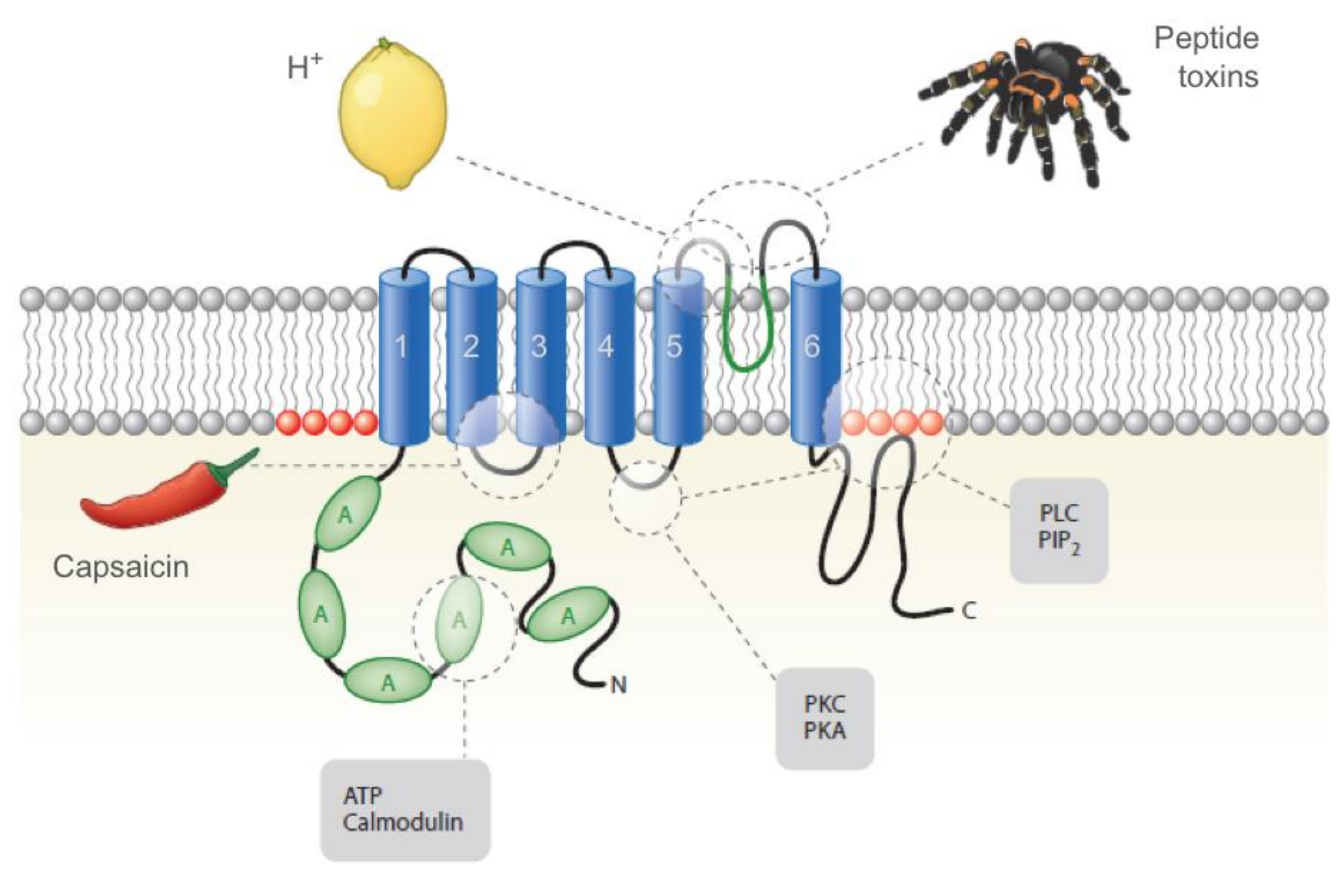

Figure 1.4: Structure and interaction sites of a TRPV1- subunit

A functional TRPV1 ion channel consists of 4 of the displayed subunit. Each of the subunits comprises 6 transmembrane domains (TMD) with a pore loop between TMD 5 and 6, and intracellular N- and C-termini. The N-terminus contains 6 ankyrin repeats (A). Examples of stimuli activating or modulating TRPV1 are displayed together with their interaction sites. Adapted from (Julius, 2013).

In vivo, TRPV1 seems to predominantly form homo-tetramers. Co-expressing TRPV1 in heterologous expression systems along with TRPV2 or TRPV3 leads to assembly of heteromers, yet, to a lesser degree than homo-tetramers (Hellwig et al., 2005; Smith et al., 2002). One study suggested the formation of TRPV1-TRPV2 heteromers at a low level also in rat DRG (Rutter et al., 2005). Any physiological relevance of this heteromer formation however, might be minor in vivo given that TRPV1 and TRPV2 have been shown to be mostly expressed in non-overlapping populations of sensory neurons (Ahluwalia et al., 2002; Caterina et al., 1999). 


\subsubsection{Tissue expression of TRPV1}

TRPV1 is predominantly expressed in small- to medium-diameter neurons of the peripheral sensory and sympathetic ganglia giving rise to $\mathrm{C}$-fibres, both the non-peptidergic and peptidergic (i.e. SP- and CGRP-expressing) population, and to lesser degree to A $\delta$-fibres (Cavanaugh et al., 2009; Helliwell et al., 1998; Tominaga et al., 1998; Yu et al., 2008).

TRPV1 expression in the peripheral nervous system (PNS) was also reported in sensory nerve fibres innervating airways/lung (Kollarik and Undem, 2004) and in the urinary tract/ bladder (Avelino and Cruz, 2006). Non-neuronal tissues with TRPV1 expression are the epithelium of the gastrointestinal tract (Ward et al., 2003), the cardiovasculature (Peng and $\mathrm{Li}, 2010$ ), and in the skin epidermis (Inoue et al., 2002).

Apart from the PNS, TRPV1 is also expressed in the CNS albeit to a much lower extent. Amongst others, presynaptic TRPV1 in the DH of the SC has been reported to contribute to modulation of synaptic transmission in the laminae I, II under pathological pain conditions (Spicarova et al., 2014).

A multitude of different methods with varying outcomes has been used to explore the expression of TRPV1 in the brain. In general, TRPV1 expression has been reported in diverse regions and nuclei, amongst others, the hypothalamus, thalamus, entorhinal cortex, and hippocampus, several parts of the rhombencephalon as well as mesencephalon (Cavanaugh et al., 2011; Cristino et al., 2006; Gibson et al., 2008; Musella et al., 2009; Tóth et al., 2005). Yet, the physiological function of TRPV1 in the different brain areas remains poorly understood (Kauer and Gibson, 2009). For instance, Marsch et al. proposed a role for TRPV1 in synaptic plasticity in the hippocampus (Marsch et al., 2007). Trpv1-deficient mice exhibited reduced sensitized and conditioned fear as well as anxiety and ex vivo, a decrease in long-term potentiation in a hippocampal circuit important for learning and memory was observed (Marsch et al., 2007).

\subsubsection{Agonists of TRPV1}

TRPV1 was the first TRP channel identified to be intrinsically heat-sensitive (under physiological conditions $>42^{\circ} \mathrm{C}$, (Tominaga et al., 1998)). In addition, TRPV1 is responsive to a wide range of exo- and endogenous ligands - mostly irritant chemicals and endogenous allogenic molecules. Besides capsaicin, also other irritant natural compounds, for instance, piperine (black pepper), allicin (garlic), camphor, gingerol (ginger) and eugenol (clove oil) can activate TRPV1. Also, toxins from plant (resiniferatoxin), spiders, snakes, and jelly fish are amongst the agonists of TRPV1 (Julius, 2013).

Many of the endogenous activators are derivatives of arachidonic acid that are synthesized by the body under inflammatory conditions and injury. Examples are prostaglandins, leukotriens and endocannaboids such as anadamide or $\mathrm{N}$-arachidonoyldopamine (Vriens et al., 2009). Local tissue acidosis, as seen under inflammation, ischemia and tumour growth can in extreme cases activate TRPV1 $(\mathrm{pH}<6.0)$ and under more moderate conditions $(\mathrm{pH}$ 6.5) enhance sensitivity to heat and other agonists. This dual effect was found to be mediated by protons interacting with different extracellular residues of TRPV1 (Jordt et al., 2000; Ryu 
et al., 2007). The proton-mediated sensitization leads to a shift in the heat threshold of TRPV1 to body temperature (Julius, 2013).

While the application of capsaicin is known to evoke pain in humans, topical application of low concentration capsaicin creams is well-established as an analgesic. Recently, QUTENZA $^{\text {TM }}$ (Acorda Therapeutics, Inc., Ardsley, NY, USA; Astellas Pharma Europe Ltd., Chertsey, Surrey, UK), a cutaneous patch with $8 \%$ capsaicin has been approved by the FDA for the treatment of peripheral neuropathic pain other than diabetic origin, e.g. post-herpetic neuralgia and HIV neuropathy. Local application of a high dose of capsaicin is suggested to lead to a degeneration of TRPV1-expressing intra-epidermal nerve fibres due to excessive activation of TRPV1. Studies in healthy volunteers showed that the die-back of the fibres is reversible. Unwanted side effects are minor and mainly related to the application of the capsaicin to the skin. (Uçeyler and Sommer, 2014)

Thus, QUTENZA ${ }^{\mathrm{TM}}$ is a promising example for the effectiveness of targeting peripheral expressed TRPV1 for treatment of neuropathic pain.

\subsubsection{Modulation of TRPV1 function and surface expression}

\subsubsection{Sensitization of TRPV1}

Within the plethora of pro-algesic mediators released upon tissue damage and subsequent inflammation, many are able to modulate TRPV1 function, which underlies enhanced nociceptor excitability and ultimately contributes to hyperalgesia. Many of the inflammatory mediators modulate TRPV1 indirectly via stimulation of their own receptors expressed by the nociceptor and act on the channel via activation of second messenger cascades. This often results in the phosphorylation of TRPV1 with alterations either in the open probability (i.e. the activation threshold) of the channel or translocation of the channel to the plasma membrane. (Julius, 2013)

For instance, the inflammatory mediator bradykinin (Bk) is critical to TRPV1 sensitization under inflammatory conditions. If injected intradermally it elicits profound thermal hypersensitivity, which is impaired in Trpv1-KO mice (Chuang et al., 2001). Bk binds to its $\mathrm{G}_{\mathrm{q}}$-coupled Bk1 receptor causing ultimately an activation of $\mathrm{PKC} \varepsilon$, which in turn phosphorylates TRPV1 at serine 502/800 (Bhave et al., 2003). Also, prostaglandin E2 (PGE2)-binding to its $\mathrm{G}_{\mathrm{s}}$-coupled EP4 receptor leads to a phosphorylation of TRPV1 in this case by PKA at serine 116 (Mohapatra and Nau, 2003). Phosphorylation mediated by the PKC are thought to mainly result in an increase of the open probability of the channel at standard membrane potentials (Bhave et al., 2003; Matta and Ahern, 2007; Numazaki et al., 2002; Vellani et al., 2001). In a recent study by Mathivanan et al., however, bradykinin was also found to increase the surface trafficking of TRPV1 (Mathivanan et al., 2016). Sensitization mediated by PKA are rather thought to reverse the desensitization state of the channel ((Bhave et al., 2002), see below).

Moreover, phosphorylation by kinases acting downstream of the NGF receptor TrkA results in increased translocation of the TRPV1 to the plasma membrane (Stein et al., 2006; Zhang et al., 2005). How exactly phosphorylation of TRPV1 promotes trafficking to the plasmamembrane remains elusive. The interaction of TRPV1 with other proteins, for instance, the 
GABAA receptor associated protein (Laínez et al., 2010) as well as several SNARE-proteins (Camprubí-Robles et al., 2009; Morenilla-Palao et al., 2004) has been shown to regulate TRPV1 surface-expression.

\subsubsection{Desensitization of TRPVI}

An interesting feature of TRPV1 is its desensitization, a reduction in channel activity, which occurs during prolonged or repetitive stimulation of the channel with capsaicin or other agonists and might underlie the paradoxical analgesic properties of capsaicin application. The increase in intracellular free calcium upon channel activation has been reported to trigger this (mostly) reversible channel inactivation preventing a cytotoxic calcium overload of the nociceptor (Koplas P. A. et al., 1997). Yet, it should be noted that upon removal of extracellular calcium in patch clamp experiments, the channel desensitization is not completely abolished (Koplas P. A. et al., 1997; Mohapatra and Nau, 2003).

Several mechanisms have been proposed for the calcium-mediated desensitization. One is that the incoming calcium binds to calmodulin $(\mathrm{CaM})$, which then activates the $\mathrm{Ca}^{2+} / \mathrm{CaM}$ dependent phosphatase calcineurin (Docherty et al., 1996). Calcineurin in turn dephosphorylates TRPV1 (Mohapatra and Nau, 2005). This is underscored by studies showing that the calcium-dependent desensitization can be reduced/reversed by PKA- and PKC-mediated phosphorylation of several sites (Bhave et al., 2002; Mandadi et al., 2004, 2006; Mohapatra and Nau, 2003; Numazaki et al., 2002) . On the other hand, $\mathrm{Ca}^{2+} / \mathrm{CaM}$ was shown to interact with the ankyrin-repeats of the N-term of TRPV1 to trigger desensitization (Lishko et al., 2007; Rosenbaum et al., 2004). Also, the calcium-mediated depletion of phosphoinositides has been reported to contribute to the inhibition of channel activity after capsaicin stimulation (Liu, 2005; Lukacs et al., 2013).

The long-term desensitization of TRPV1-expressing nociceptors has been suggested to be accompanied by alteration in the level of plasma membrane-resident TRPV1: Sanz-Salvador et al. reported a rapidly induced endocytosis of TRPV1 in a time- and dose-dependent matter with subsequent degradation in lysosomes (Sanz-Salvador et al., 2012).

\subsubsection{Involvement of TRPV1 in pain conditions}

Early reports on the pain-inducing properties of capsaicin application in rodents and humans and the seemingly paradoxical analgesic effect of prolonged capsaicin exposure (e.g. (Carpenter and Lynn, 1981)) supported a potential role for the capsaicin receptor in pain signalling in vivo long before its molecular identification.

Whilst capsaicin-induced nocifensive behaviour was abolished in Trpv1-KO mice, they did not show profound deficits in noxious heat-evoked nociceptive behaviour suggesting further molecules to be involved in the detection of heat in vivo (Caterina et al., 2000; Davis et al., 2000). However, the development of thermal hyperalgesia after inducing inflammatory pain was greatly impaired in Trpvl-KO mice. This is the case for both acute thermal hypersensitivity after hind paw injection of bradykinin (Chuang et al., 2001) as well as complex inflammatory pain paradigms induced by Complete Freund's Adjuvant (CFA) or carrageen injection (Caterina et al., 2000; Davis et al., 2000). In skin, sciatic nerve, DRG 
and SC TRPV1 protein and/or mRNA was found to be upregulated upon inflammation (Amaya et al., 2003; Carlton and Coggeshall, 2001; Ji et al., 2002; Tohda et al., 2001).

Whilst in the case of the global knockout of Trpvl only thermal hyperalgesia was attenuated, the systemic administration of TRPV1 antagonists not only attenuated thermal but also mechanical hyperalgesia in the CFA model of inflammatory pain, which could be explained by developmental compensation in the global Trpv1-KO (Gavva et al., 2005; Kanai et al., 2007; Pomonis et al., 2003; Walker et al., 2003).

The role of TRPV1 in a wide range of neuropathic pain models - from painful diabetic neuropathy to nerve transection/ligation models - has been investigated intensively. However, its contribution to neuropathic pain is less well understood than in painful conditions linked to inflammation (Mickle et al., 2016). Several studies reported alterations in the expression profile of TRPV1 in DRG neurons. Interestingly, most report an overall downregulation of TRPV1 in the DRG after nerve injury (Hudson et al., 2001; Schäfers et al., 2003). However, when distinguishing between damaged and non-damaged neurons, TRPV1 was found to be upregulated in the uninjured nerve fibres/neurons and downregulated in the injured ones (Fukuoka et al., 2002; Hudson et al., 2001; Kim et al., 2008). Consequently, RNAi-mediated knockdown as well as antagonists of TRPV1 diminished stimulus-evoked pain hypersensitivity in models for the partial ligation and injury of the sciatic nerve (Christoph et al., 2006, 2008; Kanai et al., 2005; Pomonis et al., 2003).

\subsection{Targeting protein-protein interactions for pain relief}

As exemplified with the occurrence of adverse side effects when blocking TRPV1 activity for pain relief, the direct targeting of nociceptive ion channels, and TRPV1 in particular, may not be the ideal starting point for developing novel analgesics. Therefore, researchers look for alternative strategies to inhibit only the pathophysiological hyperactivity of the ion channel. One alternative is to look for state-dependent antagonists, yet, another promising one might be instead of targeting TRPV1 directly, to prevent crucial TRPV1 interactions that only take place under pathological pain conditions. (Fernández-Carvajal et al., 2012)

Proteins fulfil their diverse function in a cell not as "lone warriors". They assemble into multiprotein complexes - known as "protein machines" (Alberts, 1998) - enabling them to fulfil critical tasks, for instance, transmembrane signal transduction. Only the dynamic interactions between the involved proteins ensure the correct activity and specificity of the complexes. However, protein-protein interactions (PPIs) could occur at a wrong time and duration, or location, or due to a loss of an interactor not take place at all (Zinzalla and Thurston, 2009). Aberrant PPIs have been discovered to be the molecular basis of many diseases, for instance, neurodegenerative diseases (Ryan and Matthews, 2005).

Targeting disease-specific PPIs, without interfering with other functions of the involved proteins, has great potential to yield highly selective drugs. The high tissue selectivity and modifying cellular function only in a subtle way should allow for minimal side effects 
(Blazer and Neubig, 2009). This would be highly desirable for chronic pain therapeutics as the currently available treatment options are only effective for a minority of cases, and are accompanied by severe side effects limiting the use of an effective dosage.

Receptors and ion channels have a unique position at the very beginning of the transmission of pain messages. Since they show dysregulated expression and alterations in their intrinsic channel properties during pathological pain states, interfering with the responsible PPIs may offer an attractive strategy for developing novel analgesics. The interference could be both stabilizing in case of analgesic PPIs, or if a PPI promotes the excessive activation of a nociceptive ion channel, the PPI would be prevented. In recent years, the modulation of PPIs for several ion channels/receptors involved in pain signalling has been shown to alter pain perception in rodents. (Rouwette et al., 2015)

For instance, a seminal study by Liu and colleagues showed that uncoupling the Scr kinase from the NMDA receptor complex led to analgesia in both models of inflammatory and neuropathic pain (Liu 2008). Activity of NMDA receptors underlies neuroplasticity and is key to pain hypersensitivity, yet, blocking these channel for pain relief is deleterious as they fulfil important physiological functions in the CNS (Lynch and Watson, 2006). The Src kinase is known to enhance NMDA function via phosphorylation and is anchored within the NMDA receptor complex (Salter and Kalia, 2004). Liu et al. designed a peptide mimicking the binding interface of Scr and its adaptor protein within the NMDA complex to disrupt the interaction in vivo. Intrathecal and intravenous administration of the cell-permeable peptide attenuated pain behaviours in rodents, not only in models for inflammatory pain, but also in peripheral nerve injury. Remarkably, uncoupling the interaction did not affect cognitive functions, locomotion or basal pain thresholds (Liu et al., 2008). This study highlights the potential of targeting ion channel complexes to achieve analgesia with minimized side effects.

Also, TRPV1 (mal)functioning is critically shaped by PPIs. In the following, a few examples for interactions whose relevance has been shown for TRPV1-mediated nociceptive signalling in vivo are given.

\subsubsection{AKAP79/150}

AKAP79/150, a member of the A-Kinase anchoring protein family (AKAP), is a scaffolding protein with binding sites for TRPV1 as well as the kinases PKC and PKA that are activated downstream of cellular signalling cascades initiated by the inflammatory mediators Bk and PGE2 and ultimately sensitize TRPV1 (Btesh et al., 2013; Jeske et al., 2008, 2009). Preventing the binding of either the kinases or TRPV1 to AKAP79/150 has been shown to abolish the sensitization of TRPV1 by Bk and/or PGE2 not only in vitro but also in vivo: Schnitzler et al. reported reduced PGE2-induced thermal hyperalgesia in geneticallymodified mice missing the PKA binding-site of AKAP79/150 compared to wild type mice (Schnizler et al., 2008). Subsequently, Fisher and colleagues used a peptide mimicking the binding site of TRPV1 and AKAP79/150 to uncouple their interaction in vivo and could thereby reduce thermal hypersensitivity in two inflammatory pain models. Notably, basal pain thresholds in the absence of inflammation were unchanged (Fischer et al., 2013). 


\subsubsection{GABA $A_{B 1}$ receptor}

Recently, Hanack et al. reported the physical and functional interaction of TRPV1 and the $\mathrm{GABA}_{\mathrm{B} 1}$ receptor subunit that counteracts the hyperactivity of TRPV1 under pathological pain conditions, yet does not interfere with acute TRPV1-mediated pain (Hanack et al., 2015): The authors could show that an autocrine feedback mechanism exists in which the neurotransmitter GABA is released from peripheral nociceptive terminals upon activation of TRPV1 and stimulates the $\mathrm{GABA}_{\mathrm{B} 1}$ subunit triggering a non-canonical signalling pathway that in the end reverts TRPV1 sensitization. A robust decrease in heat hyperalgesia was observed in several inflammatory pain paradigms in mice treated with the GABA baclofen. The administration of baclofen did not affect acute capsaicin-evoked nocifensive behaviour or the basal thermal pain threshold. (Hanack et al., 2015)

This shows that the activation of $\mathrm{GABA}_{\mathrm{B} 1}$ modulates selectively the excessive TRPV1 activity occurring under pathological conditions and consequently, offers an interesting mechanism to interfere with TRPV1-mediated hypersensitivity.

\subsubsection{TMEM100/TRPA1}

A subgroup of TRPV1-expressing nociceptors co-expresses the TRPA1 channel (Bautista et al., 2005; Story et al., 2003) and several studies have suggested a physical and/or functional interaction of both channels (Akopian et al., 2007; Salas et al., 2009; Staruschenko et al., 2010). TMEM100, a membrane adaptor protein has been shown to be able to bind both channels and thereby regulate the association of TRPA1 and TRPV1 (Weng et al., 2015): The genetic ablation of Tmem100 in mice led to a reduction in TRPA1-mediated but not TRPV1-mediated acute pain model as well as to attenuated mechanical hyperalgesia upon inflammation - for whose development TRPA1 is thought to be more relevant than TRPV1. Mechanistically, TMEM100 selectively potentiates the activity of TRPA1 by relieving it from the inhibitory effect TRPV1 has been proposed to exert on TRPA1. Strikingly, a mutant of TMEM, TMEM100-3Q, was shown to exert the opposite effect, i.e. stabilizing the TRPV1-TRPA1 complex and therefore, decreased TRPA1 activity in a TRPV1-dependent manner. Injection of a cell-permeable mimicking peptide of the mutant form led to a reduction in TRPA1-mediated nocifensive behaviour and hypersensitivity in wild type mice, but not in Trpv1-deficient mice. Additionally, in TRPV1-mediated pain paradigms no attenuation was observed in treatment with the mimicking peptide showing that the analgesic effect is selective for TRPA1 and depends on TRPV1. (Weng et al., 2015)

The above stated interactions and their successful manipulation for pain relief, demonstrate that fine-tuning of pain-specific interaction might offer a better strategy for interfering with chronic pain than completely suppressing the activity of certain key players in pain signalling. But how to detect pain-specific interactions most efficiently with a low false positive rate? In the last few years, the method of choice for detecting PPIs has become "functional proteomics" (Schulte et al., 2011), which is the combination of affinity-based co-purification of the target protein ("bait") and associated proteins ("preys") from native tissue and tandem mass spectrometry for the identification of the co-purified interactors. 


\subsection{Mass spectrometry as a tool for the identification of pain-related proteins and protein complexes}

The quest for potentially "druggable" key player in chronic pain is dependent on the identification of proteins that show a clear regulation between the physiological and pathophysiological state (Antunes-Martins et al., 2013). Several large-scale gene expression profiling studies have been conducted of nociceptors and of animal models of pathological pain, and gave insight into significant pain-related changes of the transcriptome (Alvarado et al., 2013; Lacroix-Fralish et al., 2011; Manteniotis et al., 2013; Usoskin et al., 2015). However, the changes on mRNA level are not translated 1:1 to the protein level but are estimated to account for only $40 \%$ of the variability in protein levels (Schwanhäusser et al., 2011). Therefore, direct exploration of the proteome might be better suited to capture disease-related changes of the proteome and discover key players in pathological pain. Liquid chromatography-coupled tandem mass spectrometry (LC-MS/MS) is an analytical technique that allows large-scale identification of proteins in complex native biological samples (Aebersold and Mann, 2003), and has proven its ability to elucidate protein complexes and signalling pathways crucial for different cellular functions as well as pathological processes (Choudhary and Mann, 2010; Riley et al., 2016). Most commonly, so called "shotgun" mass spectrometry is used for large-scale proteomics studies. In Fig. 1.5, an outline of a classical "shotgun"/discovery-based tandem MS experiment is given:
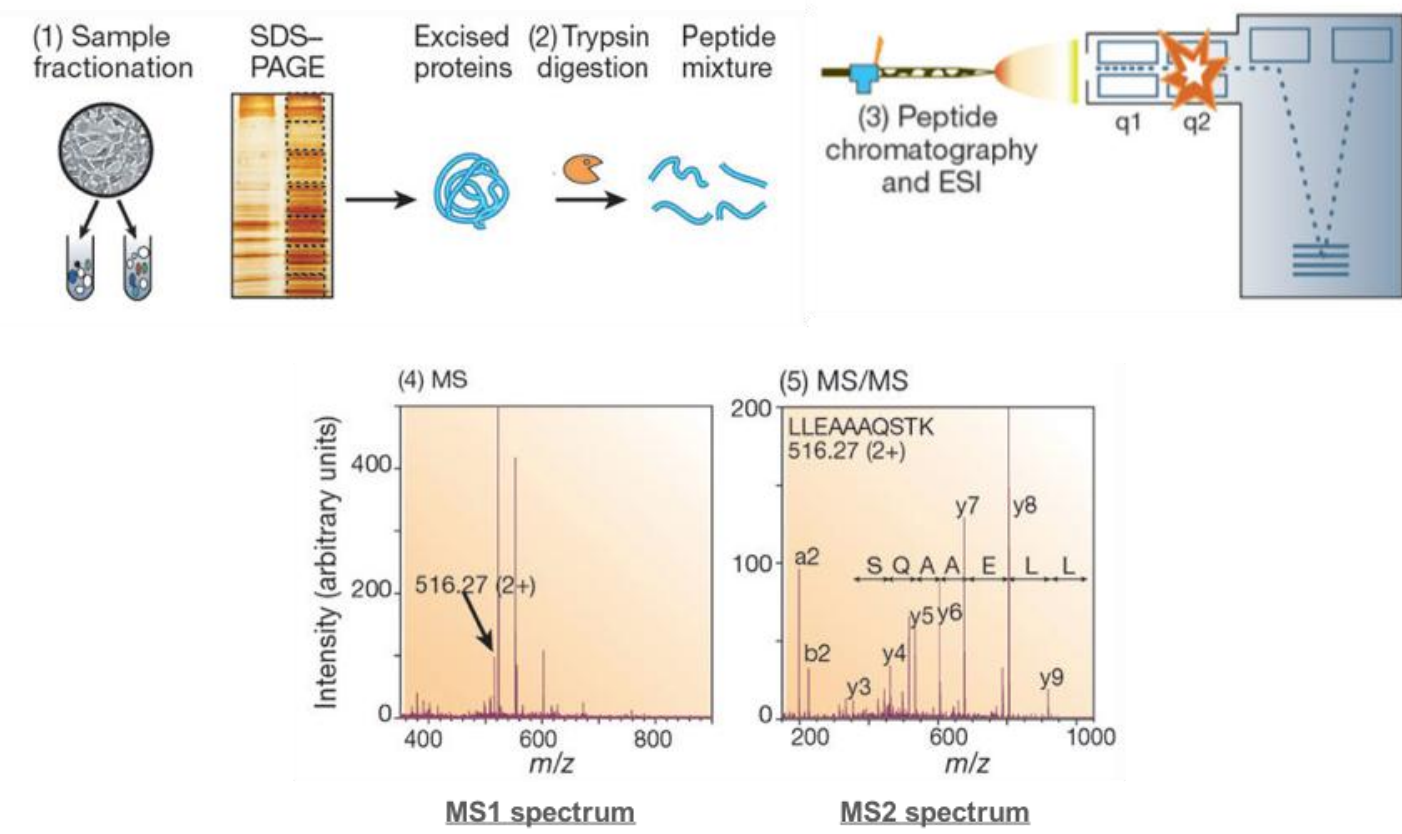

Figure 1.5: Outline of the different steps of a typical proteomics experiment

Adapted by permission from Springer Nature: Nature, Mass spectrometry-based proteomics. Aebersold and Mann, 2003.

The proteins to be identified are isolated from the tissue or cell types of interest - both crude cell lysate or less complex samples such as enriched subfractions of certain cellular components or proteins can be subjected to MS (1). Endoproteases, most commonly trypsin, digest the proteins in the sample into shorter peptides (2) that are then separated by LC to 
regulate the flow of peptides into the on-line coupled mass spectrometer. Before entering the mass spectrometer, the peptides become ionized and evaporated by electrospray ionization (ESI; 3). In the first stage of mass analysis, a mass spectrum (MS1; the mass analyser acquires the mass to charge ratio $(\mathrm{m} / \mathrm{z})$ of the peptides) is acquired from the peptides ions entering the mass analyser at a given point in time. Subsequently, the computer generates a list of peptides that are then isolated and fragmented; commonly the fragmentation is induced by collision with inert gas molecules. From the fragment ions another mass spectrum (MS2) is captured. In a final step, the peptides and consequently the proteins contained in the sample are identified by matching the acquired spectra with protein sequence databases. (Aebersold and Mann, 2003)

Shotgun proteomics has also been applied to several pain models of chronic pain, as well as tissue samples from human chronic pain patients to identify candidate proteins for chronic pain conditions (Huang et al., 2008b; Melemedjian et al., 2013; Niederberger and Geisslinger, 2008; Vacca et al., 2014; Zou et al., 2012). However, the lists of proteins regulated only show sparse overlap (Gomez-Varela and Schmidt, 2016). On the one hand, this could be attributed to differences in sample preparations. On the other hand, in most of the mentioned studies the mass spectra were acquired in the data-dependent acquisition (DDA) mode, which is common in shotgun proteomics. This acquisition technique however, comes along with some inherent technical constraints (Law and Lim, 2013): In DDA, after acquisition of a MS1 survey scan, only a limited number of the peptide ions (precursor ions) are selected for the fragmentation and the subsequent serial fragment ion scans (which are necessary for the identification and quantification). The selection is done in real-time by the mass spectrometer with a preference for the most abundant precursor ions of each MS1 scan. Furthermore, the number of selected precursor ions is restricted by the instrument's cycling time, which determines the number of possible MS2 scans. Thus, as long as the number of detected precursor ions exceeds the number of available MS2 scan cycles, the set of peptides identified across different samples of an experiment is not reproducible (Domon and Aebersold, 2010). Tabb et al. reported that the overlap between technical replicates of an average LC-MS/MS experiment ranges from 35-60\% (Tabb et al., 2010). Also "undersampling" can become problematic in an average DDA-MS experiment given that the fraction of all peptides being identified in a given sample can be as low as 10-20\% (Michalski et al., 2011). Therefore, the semi-stochastic nature of DDA peptide sampling limits its use for the exact and reproducible quantification of 1000s of proteins across many highly complex samples such as tissue lysates (Law and Lim, 2013).

In recent years, researchers have developed methods to overcome the limitations of DDA. They are based on an unbiased "data independent acquisition" (DIA). In DIA-MS cyclic scans are performed throughout the LC run after each survey scan. The parallel fragmentation of all detectable precursor ions within sequential isolation windows ranging from a few $\mathrm{m} / \mathrm{z}$ to the entire mass range is triggered. Dependent on the number of chosen isolations windows a respective number of MS2 spectra are collected after each survey scan. (Law and Lim, 2013) 
For each of the scans both the acquired MS2 spectra (m/z vs. intensity) and also the retention time are collected and stored in digital data maps. The spectra are highly complex as a single spectrum comprises all the fragment ions of several precursors ions fragmented in parallel. This chimeric nature of the spectra makes it difficult to use the traditional database search approach for the identification of the peptides. In one implementation of DIA, SWATH-MS (Sequential Windowed Acquisition of All THeoretical fragment ion mass spectra; (Gillet et al., 2012)), this problem is circumvented by the use of sample-specific spectral libraries. These reference libraries are compiled from previously acquired and analysed MS2 spectra from several highly optimized DDA runs of the sample of interest. They contain the fragment ion signals, their relative intensities and their chromatographic behaviour, all information needed to uniquely identify and quantify a peptide (Schubert et al., 2015). Accordingly, the unknown peptides signatures stored in the data maps can then be identified by looking for the best match in the library. The targeted search of spectral libraries allows the standardized and accurate quantification of a large number of proteins across many samples and laboratories (Collins et al., 2017). Furthermore, the availability of spectral libraries from a multitude of different large-scale experiments is ever increasing and offers the possibility to re-query the digitally-stored peptide signatures with any spectral library of interest to test new hypotheses in silico (Law and Lim, 2013).

DIA-MS has already been successfully applied to determine how protein abundances, modifications and interactions change across disease states (Bruderer et al., 2015; Lambert et al., 2013; Liu et al., 2014; Surinova et al., 2015) and therefore holds the promise to be of use in deciphering pain-related changes of the proteome. 


\subsection{Aims of the study}

Chronic pain imposes substantial challenges to medical practice as the treatment options for its clinically relevant manifestations are limited. Owing to lack of knowledge about the exact molecular mechanism underlying pathological pain conditions, currently available pain therapeutics target molecules with key physiological functions in our body. Thus, they are accompanied by severe side effects limiting effective dosage prescription. In the light of these difficulties, identification of proteins and their associated complexes that exert their function in the first line of noxious stimulus detection, and are differentially regulated among pain states, is highly desirable to develop better analgesics. The goal of this study was to identify and characterize multiprotein complexes in the context of nociception in mice. To this end, the study was divided into two major lines of research:

(I) Characterization of the differential regulation of membrane proteins in sensory neurons in different models of chronic pain, using emerging DIA-MS to allow for standardized and reproducible quantitation of the DRG membrane proteome. Given the essential function of membrane proteins, especially ion channels and receptors, in nociceptive signalling, defining their differential regulation is key for understanding nociception in general and related pathophysiological processes like chronic pain.

(II) Investigation of the scaffold of proteins interacting with the ion channel TRPV1 under inflammatory pain. TRPV1 channels serve as one of the major noxious stimulus detectors in sensory neurons and are crucial for the development of several pain modalities, rendering TRPV1 a promising target for novel analgesics. Despite intensive study of TRPV1, little is known about proteins interacting with TRPV1 selectively under pathological pain conditions.

\subsection{Contribution of co-workers to this study}

- Dr. Roland Bruderer (Biognosys AG, Zuerich, Switzerland): DIA-MS acquisition and quantitative data analysis including statistics for project I (see section 2.1.7.1).

- Dr. Olaf Jahn (Proteomics group, MPIem): MS acquisition and first steps of the quantitative analysis (see section 2.1.7.2) for project II.

- Dr. Sebastian Kuegler (UMG, Göttingen) produced the virus particles for project II.

- The Master students Reham Abdelaziz and Allison Barry (both IMPRS Neurosciences) performed some experiments for the project II under the author's teaching and supervision. These have been reported in their Master theses.

- Niklas Michel (Ph.D. student in my host lab) performed the electrophysiological recordings. 


\section{Material and Methods}

\subsection{Methodology}

Methods sections marked with * have been used for part I and are based on Rouwette et al., 2016. Parts marked with \# have been used for part II and most of the sections are based on Sondermann et al., 2019.

\subsubsection{Animals}

Male adult C57B1/6J mice, 6-8 weeks old at the time of injection or surgery, were used for all experiments.*

Adult C57Bl/6J mice, 8-10 weeks old at the time of treatment, were used (in the following referred to as wild type (WT) mice). In addition, Trpv1-knockout (KO) B6.129X1-

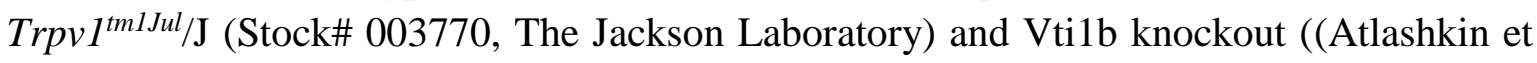
al., 2003), kind gift from G. Fischer von Mollard, Bielefeld, Germany) mice, 8-10 weeks old at the time of treatment were used. Except for the behavioural testing of Vtilb knockout mice, only male mice were used for experiments. \#

All mice were in-house bred and kept in a humidity- and temperature-controlled environment under a $12 \mathrm{~h}$ light/dark cycle. They had access to food and water ad libitum. All animal experiments were approved by and carried out in compliance with institutional guidelines as well as the guidelines of the Landesamt für Verbraucherschutz und Lebensmittelsicherheit of Lower Saxony, Germany.*\#

\subsubsection{Biochemistry}

\subsubsection{Preparation of membrane-enriched fractions of lumbar DRG*}

The protocol used for preparation of membrane-enriched fractions was modified from ( $\mathrm{Lu}$ et al., 2009). All buffers were prepared at the day of the experiment.

Snap-frozen ipsilateral lumbar DRG (IDRG) of 10-13 mice per condition were homogenized with help of a teflon glass douncer in a buffer with a high salt concentration $(2 \mathrm{M} \mathrm{NaCl}, 10$ $\mathrm{mM}$ Tris/HCl, $\mathrm{pH} 7.5,1 \times$ cOmplete protease inhibitor cocktail). For removal of cell debri, homogenates were centrifuged at $600 \times \mathrm{g}$ for $10 \mathrm{~min}$ at $4^{\circ} \mathrm{C}$. Supernatants were spun down at $16,000 \times \mathrm{g}$ for $15 \mathrm{~min}$ at $4^{\circ} \mathrm{C}$, and the pellets re-suspended in $100 \mu \mathrm{L}$ carbonate buffer $\left(0.1 \mathrm{mM} \mathrm{Na} 2 \mathrm{CO}_{3}, \mathrm{pH} 11.3\right)$. The samples were again centrifugated at $16,000 \times \mathrm{g}$ for $15 \mathrm{~min}$ at $4{ }^{\circ} \mathrm{C}$. This step was repeated once. Subsequently, pellets were dissolved in $100 \mu \mathrm{L}$ of $1 \%$ RapiGest in ammonium bicarbonate buffer $\left(50 \mathrm{mM}\left(\mathrm{NH}_{4}\right) \mathrm{HCO}_{3}, \mathrm{pH} 8.5\right)$ and incubated overnight at $4^{\circ} \mathrm{C}$, for solubilisation of membrane proteins. Samples were then pelleted at $16,000 \times \mathrm{g}$ for $15 \mathrm{~min}$ at $4^{\circ} \mathrm{C}$ with the supernatant equalling the membrane-enriched fraction. For precipitation of proteins, $5 \times$ the sample volume of ice-cold acetone was added to the supernatants and samples were precipitated for $2 \mathrm{~h}$ at $-20^{\circ} \mathrm{C}$, and precipitates pelleted at $14,000 \times \mathrm{g}$ for $30 \mathrm{~min}$ at room temperature (RT). Pellets were rinsed with pre-chilled $80 \%$ ethanol and again centrifuged at $14,000 \times \mathrm{g}$ for $30 \mathrm{~min}$, RT. Supernatants were discarded, and pellets air-dried. For the in-solution digest, $10 \mu \mathrm{L}$ of $1 \%$ RapiGest in ammonium bicarbonate 
buffer (ABC buffer) were added followed by $10 \mu \mathrm{L}$ of $50 \mathrm{mM}$ dithiothreitol (DTT) in 25 $\mathrm{mM} A B C$ buffer, and samples were reduced at $850 \mathrm{rpm}$ for $1 \mathrm{~h}$ at $56^{\circ} \mathrm{C}$ on a thermoshaker (Eppendorf). Next, $10 \mu \mathrm{L}$ of $100 \mathrm{mM}$ iodoacetamide in $25 \mathrm{mM} \mathrm{ABC}$ buffer were added and samples were alkylated at $850 \mathrm{rpm}$ for $1 \mathrm{~h}$ at $37^{\circ} \mathrm{C}$. To reach an enzyme to protein ratio of 1:20, $70 \mu \mathrm{l}$ of trypsin (Promega) solution in $25 \mathrm{mM} \mathrm{ABC}$ buffer were added and samples were digested overnight at $37^{\circ} \mathrm{C}$. Subsequently, samples were acidified with $20 \mu \mathrm{L}$ of $5 \%$ trifluoroacetic acid, incubated for $2 \mathrm{~h}$ at $37^{\circ} \mathrm{C}$, and spun down at $16000 \times \mathrm{g}$ for $30 \mathrm{~min}$. After lyophilisation of supernatants, samples were stored at $-20^{\circ} \mathrm{C}$ until further use. The in-solution digest was performed by Monika Raabe, MPIbpc.

In total 3 biological replicates were done for each experimental group, yielding in total 12 samples that were submitted to MS (see section 2.1.7.1)

\subsubsection{Co-immunoprecipitation (CoIP) of TRPV1 \#}

Ipsilateral IDRG of CFA- and Vehicle-injected WT mice (10 mice per biological replicate) and bilateral LDRG from Trpv1-KO mice (5 mice per biological replicate) were freshly dissected. In total 9 samples (3 biological replicates) were submitted to MS (see section 2.1.7.2).

All steps of this protocol were performed on ice or at $4{ }^{\circ} \mathrm{C}$ with pre-chilled reagents and all incubation steps under constant agitation (on a tube rotator). All buffers were prepared freshly before use. Homogenization of tissue was done with help of a glass teflon dounce homogenizer in $500 \mu \mathrm{L} /$ sample solubilisation buffer (SB; $0.1 \mathrm{M} \mathrm{NaCl}, 0.05 \mathrm{M}$ Tris- $\mathrm{HCl}$, $0.32 \mathrm{M}$ sucrose, $1 \mathrm{mM}$ DTT, $1 \%$ n-Dodecyl $\beta$-D-maltoside, $1 \times$ complete protease inhibitor cocktail) and subsequent shearing was done $5 \times$ with a $20 \mathrm{G}$ needle and $10 \times$ with a $25 \mathrm{G}$ needle. The tissue homogenate was solubilized for 1 hour and cell debris was removed by centrifugation at $2,500 \times \mathrm{g}$ for $10 \mathrm{~min}$. Tissue lysates were "precleaned" by incubating with $50 \mu \mathrm{L}$ prewashed magnetic Dynabeads Protein $\mathrm{G}$ for $1 \mathrm{~h}$ in order to decrease the unspecific binding to the affinity matrix (Dynabeads Protein $\mathrm{G}+\mathrm{IgG}$ ). Twelve micrograms of the respective antibody (see Table 2-2 for antibodies) were incubated for $4 \mathrm{~h}$. followed by addition of $50 \mu \mathrm{L}$ prewashed Dynabeads Protein G. After overnight incubation the samples were rinsed once with SB and then eluted in $40 \mu \mathrm{L}$ of $1 \times$ Tris-Glycine/SDS Sample Buffer $+1 \mathrm{mM}$ DTT at $70^{\circ} \mathrm{C}$ for $10 \mathrm{~min}$ with careful mixing every $2 \mathrm{~min}$.

In case of CoIPs from transfected HEK cells that were not submitted to MS analysis, but were analysed by immunoblot, all steps of the CoIP were done as described above, but with the following additional steps:

Transfection and harvesting of HEK293 cells: Cells were seeded on $10 \mathrm{~cm}$ dishes (5-7 per condition), and $24 \mathrm{~h}$ later they were transfected with TRPV1-YFP and/or Vti1b-mycDDK/pCMV-Sport6 plasmid (see Table 2-3 for details on plasmids). Per dish $1 \mathrm{~mL}$ of transfection mix was added ( $1 \mathrm{~mL}$ OPTI-MEM, $12 \mu \mathrm{L}$ FuGENE HD, x $\mu \mathrm{g}$ plasmid). Four micrograms of TRPV1-YFP and 4-6 $\mu \mathrm{g}$ Vti1b/pCMV-Sport6 plasmid were added per dish. The amount of Vti1b/ pCMV-Sport6 was changed from 4 to $6 \mu \mathrm{g}$ across replications with no noticeable difference, but always kept consistent between conditions for a given 
experiment. To keep DNA amounts similar between conditions the pCMV-Sport6 empty vector was added.

Forty-eight hours after transfection (cell confluency of $\pm 80 \%$ ), cells were harvested in $5 \mathrm{~mL}$ $\mathrm{PBS} /$ dish and centrifuged at $1000 \mathrm{rpm}$ for $5 \mathrm{~min}$. The cell pellet was re-suspended in $1 \mathrm{~mL}$ PBS, transferred to an Eppendorf cup, and centrifuged further for $5 \mathrm{~min}$ at $1000 \mathrm{rpm}$. The resulting pellet was re-suspended in $500 \mu \mathrm{L} \mathrm{SB}$, and processed as described above (from the solubilisation step onwards) with modifications:

- before addition of antibody, $20 \mu \mathrm{L}$ of the supernatant was saved for immunoblot analysis (equals the "input") and

- the elution buffer consisted of 1x LDS Sample Buffer $+1 x$ Reducing Agent in SB.

\subsubsection{Gel-electrophoresis and western blot*\#}

Protein samples were dissolved in 1x LDS Sample Buffer $+1 \mathrm{x}$ Reducing Agent and heated to $70^{\circ} \mathrm{C}$ for $10 \mathrm{~min}$ followed by separation via 1D NuPAGE® (Life Technologies). As a marker for protein size Novex Sharp Pre-stained Protein Ladder was used. Pre-casted NuPAGE® Bis-Tris 4-12\% gradient gels were loaded with the proteins and electrophoresis was done in $1 \times$ NuPAGE® MOPS SDS running buffer. The voltage used was $200 \mathrm{~V}$ and running time varied, depending on sample separation, from 40-50 min. Subsequently, proteins were transferred to PVDF membranes with the iBlot ${ }^{\circledR}$ Gel Transfer Device (Life Technologies, program \#3, transfer time $7 \mathrm{~min}$ ). For blocking of unoccupied binding sites of the membranes, they were incubated with $5 \%$ milk/PBS for $30 \mathrm{~min}$ at RT. Next, the blots were incubated with primary antibodies (diluted in 1\% milk/PBS; see Table 2-2) for $2 \mathrm{~h}$ at $\mathrm{RT}$ and additionally at $4{ }^{\circ} \mathrm{C}$, overnight, $3-5 \times$ diluted. Membranes were washed with PBS $(3 \times$ short, $3 \times 5 \mathrm{~min}$ ), followed by incubation of respective secondary antibodies for $1 \mathrm{~h}$ at RT. After extensive washing with PBS ( $3 \times$ short, $3 \times 5 \mathrm{~min}$ ), immuno-labelled proteins were detected by near-infrared light (Odyssey; LI-COR Biosciences).

\subsubsection{Molecular Biology}

\subsubsection{Cloning of the AAV6-Vtilb miRNA construct and virus production \#}

\section{Design of microRNA (miRNA)}

The miRNA targeting mouse Vtilb was designed using the BLOCK-iT" ${ }^{\text {TM }}$ RAi Designer from Thermo Fisher Scientific (https://rnaidesigner.thermofisher.com/rnaiexpress/) and correct folding of the oligonucleotides controlled by the RNAfold WebServer (offered by ViennaRNA Web Services, http://rna.tbi.univie.ac.at/cgi-bin/RNAWebSuite/RNAfold.cgi).

The sequence of the miRNA follows the general structure:

5' TGCT overhang - G+antisense target sequence - TTGGCCACTGACT (Loop sequence)

- Sense $\Delta 2$ nt target sequence - CAGG 3' overhang.

Sequence targeted by the "miRNA 231"/Vtilb miRNA:

TAGTTTCTCAGCTTAGACATCG

Sequence of the Vtilb miRNA, flanked by a BamHI and a HindIII restriction site 
5'ggatcctggaggcttgctgaaggctgtatgctgTAGTTTCTCAGCTTAGACATCGTTTTGGCCACT GACTGACGATGTCTACTGAGAAACTAcaggacacaaggctgttactagcactcacatggaacaaatgg cccagatctggccgcactcgagatatctagacccagctttcttgtacaaagtggttgatatccagcacagtggaagctt 3'

The oligonucleotide was synthesized and inserted into a pUC57-Kan plasmid via the BamHI and HindIII sites by GenScript Inc. USA (Piscataway, NJ, USA)

\section{Cloning of the AAV6-Vtilb miRNA-EGFP construct}

The AAV6-control miRNA vector used as backbone plasmid was a kind gift from Sebastian Kügler (UMG, Göttingen) and contained one cassette with co-cistronic expression of EGFP and a negative control miRNA with random sequence under the neuron-specific promotor human synapsin 1 as well as a WPRE (woodchuck hepatitis virus posttranslational control element) element (Sousa-Ferreira et al., 2011).

Both the AAV6 vector as well as the pUC57-miRNA 231 were digested with FastDigest BamHI (FD0054, Thermo Scientific) and FastDigest HindIII (FD0504, Thermo Scientific) for $60 \mathrm{~min}$ at $37^{\circ} \mathrm{C}$ in $1 \times$ FastDigest Green Buffer to remove the control miRNA sequence or the pUC57-backbone, respectively. In addition, during last $20 \mathrm{~min}$ of incubation Fast Alkaline Phosphatase (EF0654, Thermo Scientific) was added. The digests were separated by agarose-gelelectrophoresis and the bands of interest excised. The DNA was purified using the NucleoSpin Gel and PCR Clean-up kit (Machery-Nagel) according to manufacturer's instructions. For the ligation the vector and insert were mixed at a 1:4 ratio and incubated with $1 \mathrm{U}$ of T4-DNA Ligase (15224-017, Life technologies) in 1×T4 DNA Ligase buffer overnight at $14^{\circ} \mathrm{C}$.

Transformation of the ligation product into $\mathrm{SURE}^{\circledR} 2$ Supercompetent Cells was done following manufacturer's instructions and cells were plated on LB agar plates (with Ampicillin) and grown overnight at $37^{\circ} \mathrm{C}$. Colonies were picked the following day and allowed to proliferate overnight in LB medium at $37^{\circ} \mathrm{C}$. Subsequently, isolation of plasmid DNA was carried out using PureLink ${ }^{\circledR}$ silica membrane columns that selectively bind plasmid DNA (PureLink ${ }^{\circledR}$ Quick Plasmid Miniprep Kits) following manufacturer's instructions and stored at $-20^{\circ} \mathrm{C}$ until further use.

Sequencing of generated plasmid verified the correct insert sequence (sequencing was done in-house: AGTC lab, MPIem), and a SmaI (\#FD0664, Thermo Scientific) digest confirmed the integrity of ITR (inverted terminal repeats) in the AAV6-backbone vector.

\section{Production of recombinant AAV6-virus particles}

The production of viral particles was performed by Sebastian Kügler (UMG, Göttingen) and as described before (Shevtsova et al., 2005). In short, recombinant AAV6-viruses were propagated in transiently transfected HEK293 cells using pDP6 as the helper plasmid. Purification of viral particles was performed by iodixanol step gradient ultracentrifugation and affinity chromatography over a heparin column on an Äkta FPLC. After extensive dialysis of eluted particles against PBS, the purity was determined by SDS-PAGE and genome titres by qPCR. 


\subsubsection{RNA isolation, cDNA synthesis and real-time quantitative PCR (qPCR) \#}

To validate the siRNA-mediated knockdown of Vtilb in primary cultures of LDRG neurons, total RNA was extracted from neurons transfected with either Vtilb siRNA or negative control siRNA using the NucleoSpin RNA XS kit according to the manufacturer's instructions. The concentration and purity of isolated RNA was determined via photospectrometry. If necessary, RNA was stored at $-80^{\circ} \mathrm{C}$ until further use as template for cDNA synthesis.

Leftovers of genomic DNA (gDNA) were digested and cDNA was reverse transcribed from total RNA (approx. similar amounts across conditions, $\pm 250 \mathrm{ng}$ ) using the QuantiTect Reverse Transcription Kit, following recommendations of the manufacturer. If not used immediately as template for gene amplification by qPCR, cDNA was stored at $-20^{\circ} \mathrm{C}$, until further use.

Primer for the two house-keeping genes (mouse $\beta$-actin and mouse GAPDH) and mouse Vtilb were designed with the Roche Universal Probe Library Assay Design Center (see Table 2-5 for primer sequences). The real time qPCR for comparative gene expression analysis was done on a Roche Lightcycler 480 platform with SYBR ${ }^{\mathrm{TM}}$ Green fluorescence detection. For the qPCR in 384-well plates, $200 \mathrm{nM}$ of each primer (reverse and forward), up to100 ng of cDNA and $1 \times$ Power SYBR Green PCR Master Mix, containing all necessary PCR constituents, were diluted with $\mathrm{ddH}_{2} \mathrm{O}$ to reach a final volume of $20 \mu \mathrm{L}$. Samples were prepared in triplicates and a no-template control (no cDNA, but $\mathrm{ddH}_{2} \mathrm{O}$ ) and a gDNA control (no reverse trancriptase had been added at the cDNA synthesis step) were run in parallel to control for unspecific and gDNA amplification, respectively.

The following amplification protocol was used:

\begin{tabular}{|l|c|c|c|}
\hline Step & $\begin{array}{c}\text { Enzyme } \\
\text { activation }\end{array}$ & Denaturation & $\begin{array}{c}\text { Annealing/ } \\
\text { Extension }\end{array}$ \\
\hline Time & $10 \mathrm{~min}$ & $15 \mathrm{~s}$ & $60 \mathrm{~s}$ \\
\hline Temperature & $95^{\circ} \mathrm{C}$ & $95^{\circ} \mathrm{C}$ & $60^{\circ} \mathrm{C}$ \\
\cline { 3 - 4 } & \multicolumn{2}{|c|}{40 cycles } \\
\cline { 2 - 4 }
\end{tabular}

For confirmation of the specificity of the amplified products a melting curve analysis was run.

The so-called threshold cycle $(\mathrm{Ct})$ values - the cycle, in which SYBR green fluorescence was higher than the background fluorescence - correlates with the initial transcript amount and were normalized to the two reference genes, $\beta$-actin and GAPDH. Vtilb knockdown efficiency was calculated by the comparative $2^{-\Delta \Delta \mathrm{Ct}}$ method by Livak and Schmittgen (Livak and Schmittgen, 2001).

\subsubsection{Immunohistochemistry}

\subsubsection{Tissue processing for immunohistochemistry \#}

Mice were sacrified by $\mathrm{CO}_{2}$-inhalation, decapitated and $1 \mathrm{DRGs}$ 1-5 dissected. If treatment was applied to only one hind paw, ipsilateral and contralateral IDRG were collected separately. 
The tissue was fixed in $4 \%$ formalin/PBS for 3-4 h at $4{ }^{\circ} \mathrm{C}$ and cryo-protected overnight in $30(\mathrm{w} / \mathrm{v}) \%$ sucrose at $4^{\circ} \mathrm{C}$. For cutting, tissue was frozen in Tissue-Tek O.C.T. (optimal cutting temperature). Sections of $10 \mu \mathrm{m}$ thickness (step-serial mode) were cut with a cryostat (Leica), mounted on SuperFrost Plus slides and stored at $-80{ }^{\circ} \mathrm{C}$ until further use.

For the IHC of tissues from AAV6-injected mice: Transcardial perfusion of the mice prior to tissue dissection. Mice were anesthetized by intraperitoneal (i.p.) injection of a Ketamine/Xylazine solution (100 mg Ketamin + $10 \mathrm{mg}$ Xylazine (medistar) per $\mathrm{kg}$ bodyweight diluted in sterile PBS) and after loss of reflexes, the thorax was opened, and a cannula inserted into the left ventricle. The right atrium was cut immediately before starting the perfusion with ice-cold PBS. After washing out of all blood, mice were perfused with $10 \mathrm{~mL}$ of ice-cold $4 \%$ formalin/PBS. Dissected tissue was post-fixated in $4 \%$ formalin/PBS for half an hour at $4^{\circ} \mathrm{C}$ and processed as described above.

\subsubsection{Fluorescence immunohistochemistry (IHC) \#}

After thawing of frozen sections for half an hour at RT, sections were permeabilised and blocked for 30-60 min with $0.4 \%$ Triton X-100/PBS containing 5\% serum (either donkey or goat serum, dependent on host species of antibodies used). Next, primary antibodies in $0.1 \%$ Triton X-100/PBS $+1 \%$ serum were applied overnight at $4{ }^{\circ} \mathrm{C}$. After 3 washes in PBS, sections were incubated for $2 \mathrm{~h}$, RT, with the respective Alexa Fluor conjugated secondary antibodies in PBS $+1 \%$ serum and $0.1 \%$ Triton $\mathrm{X}-100$. Unbound antibodies were rinsed with PBS and sections were mounted in SlowFade ${ }^{\text {TM }}$ Gold Antifade Medium containing 4',6Diamidino-2-Phenylindole (DAPI). See Table 2-2 for used antibodies.

\subsubsection{Image acquisition and analysis \#}

For all immuno-histological studies digital images of the stained tissue sections were acquired on a Zeiss Axio Observer Z1 inverted epifluorescence microscope with either 10x or $20 \times$ air objectives or $40 \times$ and $63 \times$ oil immersion objectives. For all experimental groups the acquisition parameters were kept identical. Also, "secondary antibody-controls" (primary antibody was omitted) were imaged in parallel to the sample sections (data not shown). Conditions to be compared were processed concurrently using the same cultural/ tissue preparation. NIH ImageJ/Fiji (Schindelin et al., 2012, 2015) was used to analyse raw images. Cells were counted as positive for the immuno-label, if their mean intensity (in arbitrary units (i.a.u.)) exceeded the mean background intensity $+3 \times$ standard deviation from at least 10 randomly chosen unstained cells. For the Vtilb stainings the background was determined on sections from Vtilb-KO mice and transferred to WT tissue sections. only sections that were at least $50 \mu \mathrm{m}$ apart were considered in order to avoid double-counting of DRG neurons. Only for presentation purposes brightness \& contrast were adjusted in ImageJ and Adobe Photoshop CC2017.

The statistical analysis was carried out using GraphPad Prism 5.01 and 6.01 (San Diego, USA). If two groups were compared either the Mann Whitney test or the unpaired two-sided Student's t-test was used where appropriate; for comparison of multiple groups one-way 
ANOVA, two-way ANOVA (depending on number of variables; followed by either HolmSidak's or Bonferroni posttests), or Kruskal-Wallis test with Dunns' multiple comparison test where appropriate. $\mathrm{P}$-values that were smaller than 0.05 were considered significant and denoted with $* \mathrm{p}<0.05, * * \mathrm{p}<0.01$ and $* * * \mathrm{p}<0.001$.

\subsubsection{Cell culture, cell stainings \& in vitro functional assays}

\subsubsection{Dissociated cultures of lumbar DRG neurons \#}

For each primary IDRG neuron culture, 4 CFA- or Veh-injected WT mice were euthanized by $\mathrm{CO}_{2}$-inhalation and ipsilateral IDRGs 1-5 were dissected. In case of non-treated WT mice, IDRG of both sides from 2 mice were isolated.

Mice were decapitated and spines removed from the trunk. Next, a laminectomy was performed to remove the roof of the vertebral canal and expose the underlying spinal cord and DRG. LDRGs 1-5 were identified by using the last rib as a landmark for thoracic DRG 13 (Malin 2007) and carefully dissected. The tissue was collected in $1 \mathrm{~mL}$ serum-free media (DMEM/Hams' F12) and $1 \mathrm{~mL}$ Collagenase $(12 \mathrm{mg} / \mathrm{mL} ; 1: 1 \mathrm{ratio})$ was added. Tissue was digested for $60 \mathrm{~min}$ at $37^{\circ} \mathrm{C}, 5 \% \mathrm{CO}_{2}$ and afterwards triturated to ensure effective digestion. Papain solution $(10 \mathrm{U} / \mathrm{mL})$ in serum-free media was added, (final Papain dilution 1:10) and the cells were further incubated for $30 \mathrm{~min}$ at $37^{\circ} \mathrm{C}, 5 \% \mathrm{CO}_{2}$.

Subsequently, cells were pelleted at $1000 \mathrm{rpm}$ for $1 \mathrm{~min}$ and re-suspended in $1 \mathrm{~mL}$ DMEM/Hams' F12 with 10\% horse serum. For separation of cell from debris, first, an additional $1 \mathrm{~mL}$ media with serum was added, followed by $2 \mathrm{~mL}$ of Bovine Serum Albumin (BSA) that were carefully added under the cell suspension creating a separate layer. The $\mathrm{BSA} /$ cell suspension column was centrifuged for $10 \mathrm{~min}$ at $1000 \mathrm{rpm}$ resulting in a cell pellet and a layer of cell debris between BSA and medium.

Medium/BSA was removed and in case of cultures without transfection, the cells were then re-suspended with DMEM/Hams' F12 with serum and supplemented with growth factors (see below). Twenty microliters of the cell suspension were plated per coverslip (12 $\mathrm{mm}$ diameter, coated with Poly-D-lysine $(1.0 \mathrm{mg} / \mathrm{mL})$ and Laminin $(20 \mu \mathrm{g} / \mathrm{mL})$, then incubated for $20 \mathrm{~min}$ at $37^{\circ} \mathrm{C}, 5 \% \mathrm{CO}_{2}$ before filling up the medium to $1 \mathrm{~mL}$.

\subsubsection{Nucleofection of DRG neurons \#}

Transient transfection of DRG neurons with siRNA or plasmid DNA was done via electroporation ("nucleofection") using the 4D-Nucleofector ${ }^{\mathrm{TM}}$ System (X unit, 16-well $20 \mu \mathrm{L}$ Nucleocuvette ${ }^{\mathrm{TM}}$ Strips, Lonza AG).

The cell pellet after the BSA column was re-suspended in $36 \mu \mathrm{L}$ of P3 Primary neuron nucleofector solution with supplement (Lonza AG). The cell suspension was divided for experimental and control condition. The required amount of siRNA/plasmid (500 ng mCherry + 300 ng Vti1b/ pCMV-Sport6, or 500 nM siRNA; see Table 2-3, 2-4) was added to the cell solution (final volume $20 \mu \mathrm{L}$ ), and transferred to a cuvette for electroporation via program DC104. Afterwards RPMI medium with low calcium was added to each cuvette well (45 $\mu \mathrm{L}, \mathrm{RT})$ for recovery and cells were plated on PDL/Laminin-coated coverslips. After a 15 -min recovery period at $37^{\circ} \mathrm{C}, 1 \mathrm{~mL}$ of DMEM/Hams' F12+GFs was added per 
well. To reduce toxicity, half of the media was refreshed after $2 \mathrm{~h}$ at $37^{\circ} \mathrm{C}, 5 \% \mathrm{CO}_{2}$. Cells were used either $48 \mathrm{~h}$ after transfection for overexpression studies, and $72 \mathrm{~h}$ after siRNA transfection in various in vitro assays (see below).

Growth factor combinations used: For DRG cultures targeting TRPV1 only nerve growth factor (NGF, $100 \mathrm{ng} / \mathrm{mL}$ ) was added. In the case that TRPA1 was the object of the experiment a combination of growth factors was used: $50 \mathrm{ng} / \mathrm{ml} \mathrm{BDNF}, 50 \mathrm{ng} / \mathrm{mL}$ GDNF, $100 \mathrm{ng} / \mathrm{mL}$ NGF, $50 \mathrm{ng} / \mathrm{mL}$ NT-3, and $50 \mathrm{ng} / \mathrm{mL}$ NT-4.

\subsubsection{Human Embryonic Kidney (HEK)293 cells and transient transfection \#}

Coating of coverslips

For immunocytochemistry, HEK293 cells were plated on PDL $(1 \mathrm{mg} / \mathrm{mL})+$ Laminin (20 $\mu \mathrm{g} / \mathrm{mL}$ )-coated coverslips; in case of calcium imaging and electrophysiology on fibronectin $(50 \mu \mathrm{g} / \mathrm{mL})$-coated coverslips; or fibronectin-coated MatTek dishes for Live labelling and Proximity Ligation Assay. Coatings were done at the day of the experiment.

\section{Maintenance}

HEK293 cells were maintained in DMEM + GlutaMAX supplemented with $10 \%$ fetal bovine serum (FBS) and $1 \%$ penicillin/streptomycin (growth medium) in flasks at $37^{\circ} \mathrm{C}$ with $5 \% \mathrm{CO} 2$ (Heracell 150i, Thermo Scientific). The cells were split once a confluency of $\pm 80 \%$ was reached: The medium was aspirated and cells were rinsed once with pre-warmed PBS. After aspiration, enough TrypLE Express to cover the complete grown surface was added and after detachment of cells, the reaction was stopped by diluting 1:10 with pre-warmed medium. For maintenance the desired cell dilutions were made and transferred to new flasks. Alternatively, cells were plated for subsequent use in experiments (see below).

\section{Transient Transfection with FuGENE HD}

Transfection was done using FuGENE HD transfection reagent according to manufacturer's instructions with a DNA: FuGENE ratio of 1:3. The transfection medium was exchanged for normal growth medium $4 \mathrm{~h}$ after transfection $\left(37^{\circ} \mathrm{C}, 5 \% \mathrm{CO}_{2}\right)$.

Amount of plasmid DNA used for the different downstream applications:

\begin{tabular}{|l|l|c|}
\hline \multicolumn{1}{|c|}{ Application } & \multicolumn{1}{c|}{ Plasmids } & Amount per dish \\
\hline \multirow{2}{*}{ PLA \& ICC } & TRPV1-YFP & $300 \mathrm{ng}$ \\
& Vtilb-myc -DDK & $500 \mathrm{ng}$ \\
\hline Live labelling, & TRPV1-YFP & $200 \mathrm{ng}$ \\
$\mathrm{Ca}^{2+}$ Imaging \& & Vtilb-myc -DDK & $200 \mathrm{ng}$ \\
Electrophysiology & & \\
\hline
\end{tabular}

To standardise DNA amounts across conditions the empty pCMV Sport6 plasmid was added. 


\subsubsection{Immunocytochemistry (ICC) \#}

All washing and incubation steps were performed under constant agitation.

Cells were washed and fixed with $4 \%$ formalin/PBS for $10 \mathrm{~min}$ at RT. Next, cells were washed $3 \times 5$ min with PBS and incubated with PBS containing $0.4 \%$ TritonX-100 and 5\% serum (donkey or goat depending on the host species of used antibodies) for half an hour at RT. Primary antibodies (AB) were diluted in antibody-solution (1\% serum and $0.1 \%$ TritonX-100 in PBS) and applied overnight at $4^{\circ} \mathrm{C}$.

After 3 short and $3 \times 5$ min washes of cells with PBS, secondary antibodies coupled to AlexaFluor dyes were incubated for $2 \mathrm{~h}$ at RT. After 3 short and $3 \times 5$ min rinses with PBS, coverslips were mounted in SlowFade ${ }^{\mathrm{TM}}$ Gold Antifade Medium with DAPI (see Table 2-2 for used antibodies). Samples were imaged with a Zeiss Axio Observer ZI using Axiovision software as described in 2.1.4.3.

\subsubsection{Proximity Ligation Assay \#}

In situ Proximity Ligation Assay (PLA; (Söderberg et al., 2006)) was performed for the detection of close proximity of $\leq 40 \mathrm{~nm}$ of TRPV1 and Vti1b; as described in (Leuchowius et al., 2011; Narayanan et al., 2016) with modifications. The Duolink® In Situ Detection Reagents Orange set was used.

Twenty-four hours after plating and/or transfection of cells on MatTek dishes medium was aspirated and cells were rinsed two times with pre-warmed PBS. Next, fixation of cells was performed with $4 \%$ formalin/PBS for 10 min at RT and washed with PBS afterwards (3x short, $3 \times 5 \mathrm{~min}$ ). To minimize unspecific AB-binding, cells were incubated with PBS containing $0.4 \%$ TritonX-100 and 5\% serum (depending on the host species of used antibodies) for $2 \mathrm{~h}$ at RT. Application of primary antibodies (in AB-solution: $1 \%$ serum and $0.1 \%$ TritonX-100 in PBS) was overnight at $4{ }^{\circ} \mathrm{C}$. The following day, cells were rinsed $2 \times$ short and $4 \times 5 \mathrm{~min}$ in Duolink Wash Buffer A (0.01 M Tris, $0.15 \mathrm{M} \mathrm{NaCl}, 0.05 \%$ Tween20; $\mathrm{pH}$ 7.4). PLA PLUS and MINUS probes were diluted 1:10 in AB-solution and incubated 20 min at RT before adding to the cells. Cells were incubated with PLA probes for $1 \mathrm{~h}$ at $37^{\circ} \mathrm{C}$. After $2 \times$ short and $4 \times 5$ min rinses with Wash Buffer A, cells were incubated with ligation mix (1:5 dilution of Ligation Stock buffer and 1:40 Ligase in $\mathrm{ddH}_{2} 0$ ) for $30 \mathrm{~min}$ at $37^{\circ} \mathrm{C}$ to ligate PLA probes. Cells were washed three times with $1 \times$ Wash Buffer A for 10 min each. For the rolling circle amplification reaction, amplification stock was diluted 1:5 with $\mathrm{ddH}_{2} \mathrm{O}$ and the polymerase 1:80. Amplification was carried out for $100 \mathrm{~min}$ at $37^{\circ} \mathrm{C}$ and to end the reaction samples were rinsed two times for $10 \mathrm{~min}$ with Wash Buffer B (0.2 M Tris, $0.1 \mathrm{M}$ $\mathrm{NaCl}, \mathrm{pH} 7.5$ ) and twice for 1 min with $0.01 \times$ Wash Buffer B. Samples were left in PBS at $4^{\circ} \mathrm{C}$ prior to imaging with a Zeiss Axio Observer Z1 using Axiovision software.

\subsubsection{Live labelling of HEK293 cells \#}

Live labelling was done as described by Schmidt et al. (Schmidt et al., 2009) with modifications. HEK293 cells were transfected with TRPV1-YFP and Vti1b-myc-DDK. Twenty-four hours after the transfection the live HEK cells were labelled with a rabbit TRPV1 antibody targeting an extracellular domain of rat TRPV1, which was diluted 1:50 in growth medium, for $20 \mathrm{~min}$ at $37^{\circ} \mathrm{C}$. Subsequently, cells were washed $5 \times$ with fresh pre- 
warmed medium and then the donkey $\alpha$-rabbit Alexa Fluor 546 secondary AB were applied 1:200 in growth medium for $10 \mathrm{~min}$ at RT. Subsequently, the cells were washed $2 \times$ with fresh medium and once with PBS, and then fixated with $2 \%$ formalin/PBS. Excessive formalin was removed by 3 subsequent PBS washes. Cells were immediately imaged at a Zeiss Axio Observer Z1.

Following the imaging, intracellular Vtilb (targeting the myc-tag) was stained: cells were washed once with PBS and permeabilized with $0.1 \%$ TritonX-100 + 5\% donkey serum in PBS for 30 min. The primary antibodies (in 5\% donkey serum/PBS) were applied overnight at $4{ }^{\circ} \mathrm{C}$. The following day, cells were rinsed $5 \times$ with PBS and incubated with respective secondary antibodies for $2 \mathrm{~h}$ at RT, diluted 1:250 in 1\% donkey serum/PBS. After 5 washes with PBS, cells were imaged immediately.

\subsubsection{Image analysis PLA and live labelling \#}

Raw images were analysed by using NIH ImageJ/Fiji (Schindelin et al., 2012, 2015), in principle as described in section 2.1.4.3 with modifications:

For PLA, the intensity of the background was estimated using the signal from three negative cells measured per image, to calculate a primary threshold per image. These primary thresholds were averaged and used across all images (within one experimental repetition). Only YFP-positive cells were considered for analysis. They were traced and their areas measured. The ratio PLA signal above threshold/ cell area (per cell) was compared between TRPV1 + Mock and TRPV1 + Vti1b conditions.

For live labelling experiments, the threshold was calculated per cell, using the unstained area between punctate.

\subsubsection{Ratiometric calcium imaging \#}

\section{Experimental procedure}

Ratiometric calcium imaging was performed as described before (e.g. (Laínez et al., 2010)) with modifications using an inverted microscope (Zeiss Axio Observer Z1). The cells either transfected DRG neurons or HEK cells - were washed four times carefully with prewarmed Assay Buffer (consisting of $1 \times$ Hanks' Balanced Salt Solution $\left(1.3 \mathrm{mM} \mathrm{Ca}^{2+}\right)$ with $10 \mathrm{mM}$ HEPES) prior to a 30-60 min incubation at $37^{\circ} \mathrm{C}$ with Fura-2/AM (stock solution: $50 \mu \mathrm{g}$ Fura-2/AM with $50 \mu \mathrm{L}$ DMSO and $50 \mu \mathrm{L}$ Pluronic F-127 Acid) diluted 1:200 in Assay Buffer (2.5 mM Fura-2/AM + 0.04\% Pluronic F-127 Acid). The duration of incubation varied depending on the length of the imaging protocol but was consistent across experimental replicates. Subsequent to incubation, cells were washed four times with prewarmed Assay Buffer and transferred to a recording chamber constantly perfused by a gravity-driven system (circa $5 \mathrm{~mL} / \mathrm{min}$ ), imaging procedure at RT. 


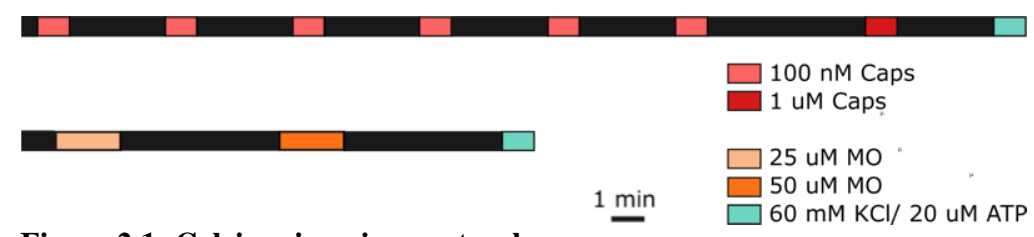

Figure 2.1: Calcium imaging protocols

Upper: TRPV1 stimulation protocol (HEK and DRG). bottom: TRPA1-DRG stimulation protocol. Caps, capsaicin; MO, mustard Oil; potassium chloride $(\mathrm{KCl})$ was used on DRG cultures, ATP used to stimulate HEK cultures. Assay buffer was applied between each pulse. Figure modified with permission from A.Barry.

Increase in intracellular calcium in response to TRP channel agonist application was studied via two imaging protocols (Fig. 2.1): (I) TRPV1 activation (DRG and HEK): six pulses of low Capsaicin (Caps; $100 \mathrm{nM})$ and a subsequent high Caps pulse $(1 \mu \mathrm{M})$, in case of HEK cells an additional ATP pulse $(20 \mu \mathrm{M})$, or (II) TRPA1 activation (only DRG): one $25 \mu \mathrm{M}$ Mustard Oil (MO) pulse and one $50 \mu \mathrm{M})$ pulse followed by an application of $\mathrm{KCl}(60 \mathrm{mM})$. Dilution of stock solutions (in absolute EtOH) of agonists in assay buffer.

Dynasore monohydrate (endocytosis inhibitor experiment) was added to the Assay Buffer from second minute of the wash out step of Caps pulse 5 until the end of pulse 6 at a final concentration of $5 \mu \mathrm{M}$.

The calcium-sensitive dye Fura-2/AM was alternately excited at $340 \mathrm{~nm}$ and $380 \mathrm{~nm}$, and emission acquired at $510 \mathrm{~nm}$, using MetaFluor software (Molecular Device). The 340/380 ratio - representing the bound/unbound calcium - describes the intracellular calcium concentration, and was sampled at $0.333 \mathrm{~Hz}$. Background fluorescence was recorded from a region devoid of cells in each of the coverslips imaged.

\section{$\underline{\text { Data analysis }}$}

Recorded data were analysed with Microsoft Excel: A baseline was calculated for each pulse as the mean of the 5 last 340/380 ratio values (ie $15 \mathrm{~s}$ before pulse) prior to pulse application. Responders: cells with amplitude values reaching $120 \%$ of the baseline within the time span from onset of stimulation until $15 \mathrm{~s}$ before the next stimulation. Amplitudes: the maximum $340 / 380$ ratio from the onset of stimulation to $15 \mathrm{~s}$ before onset of the subsequent stimulation subtracted by the respective baseline to allow for comparison across cells and cultures. Coverslips where more than $20 \%$ of cells appeared unhealthy, ie. a high initial 340/380 baseline and unstable trace recordings, were omitted from the analysis. At least two coverslips per condition from three to five independent culture preparations were analysed.

The statistical analysis was carried out using GraphPad Prism 5.01 (San Diego, USA). For the comparison of two groups either the Mann Whitney test or unpaired two-sided Student's t-test was used where appropriate; for comparison of multiple groups either one- or two-way ANOVA were performed, followed by uncorrected Fisher's LSD (least significant difference), Holm Sidak's or Bonferroni posttests. For comparison of the fractions of responders Fisher's exact test was used. P-values smaller than 0.05 were considered significant and marked with $* \mathrm{p}<0.05, * * \mathrm{p}<0.01$ and $* * * \mathrm{p}<0.001$. 


\subsubsection{Electrophysiology \#}

Electrophysiological recordings were performed by Niklas Michel, AG Schmidt.

Whole-cell patch clamp experiments were performed using an EPC10 USB amplifier (HEKA Elektronik) and the PatchMaster software (HEKA). Micropipettes were generated from borosilicate glass capillaries (PG10165-4, World Precision Instruments) using a PIP 6 (HEKA) vertical puller. The patch pipette resistance varied between 1.5 and $3 \mathrm{MOhm}$. The bath solution consisted of $160 \mathrm{mM} \mathrm{NaCl}, 2.5 \mathrm{mM} \mathrm{KCl}, 1 \mathrm{mM} \mathrm{MgCl} 2,2 \mathrm{mM} \mathrm{CaCl}, 10 \mathrm{mM}$ HEPES and $8 \mathrm{mM}$ glucose (adjusted to $\mathrm{pH} 7.4$ with $\mathrm{NaOH}$ ); pipette solution consisted of $100 \mathrm{mM} \mathrm{KCl}, 45 \mathrm{mM}$ NMDG, $10 \mathrm{mM}$ BAPTAxK$_{4}, 10 \mathrm{mM}$ HEPES and $5 \mathrm{mM}$ EGTA (adjusted to $\mathrm{pH} 7.2$ with $\mathrm{NaOH}$ ) (García-Ferreiro et al., 2004). YFP-positive cells were subjected to whole-cell voltage clamp at a holding potential of $-60 \mathrm{mV}$. Cells were subjected to linear voltage ramps from -100 to $+100 \mathrm{mV}$ with duration of $500 \mathrm{~ms}$ (de la Roche et al., 2016; Leffler et al., 2008) applied every 5 s. $100 \mathrm{nM}$ capsaicin were applied through a gravity-driven application system with a capillary, which was placed in close proximity to the cell of interest. Experiments were performed at RT. Data were analysed with the FitMaster software (HEKA).

\subsubsection{In vivo experiments}

\subsubsection{Pain paradigms and behavioural tests *\#}

Assessment of mechanical (hyper)sensitivity *\#

Mechanical (hyper-)sensitivity was determined with a Dynamic Plantar Aesthesiometer (\#37450, Ugo Basile) according to standard procedures described in (Minett et al., 2013) and the manufacturer's manual.

The mice were placed separately in clear plexiglass compartments on a mesh floor and were acclimatized for $2 \mathrm{~h}$. The hind paws were stimulated with a graded force (0-10 g in $40 \mathrm{~s})$ to the plantar surface (for SNI/Sham mice to the lateral side of the plantar surface of each hind paw) and the time until withdrawal of paw was determined (withdrawal latency). Between each stimulus on the same mouse at least 2 min passed. From at least three readings per paw the average withdrawal latency was calculated. Deviation of single values of more than $5 \mathrm{~s}$ from the median were excluded from the calculation of the average. In order to take differences in the latencies between the left and right paw prior to any treatment into account, the withdrawal latency of the left (treated) paw was also normalised to the right (untreated) one for each individual mouse and displayed in \% ipsi/con (see Appendix Fig. 7.4).

\section{Assessment of thermal (hyper)sensitivity \#}

The Hargreaves' test (Hargreaves et al., 1988) was conducted to assess the heat sensitivity of the hind paws. A Plantar Test device (\#37370, Ugo Basile, Italy) was used according to the manufacturer's recommendations and standard procedures described in (Minett et al., 2013). 
Mice were habituated for $2 \mathrm{~h}$ in clear plexiglass compartments on a clear floor. The plantar surface of each hind paw was stimulated with a heat beam from a mobile radiant heat source. The infra-red intensity of the heat source was chosen to obtain average withdrawal values of naïve wild type mouse around $10 \mathrm{~s}$ (IR 25). The cut-off time was $30 \mathrm{~s}$ to avoid tissue damage and the averages per mouse determined as described above.

\section{Acute pain-Capsaicin injection \#}

In order to assess TRPV1-mediated acute pain, nocifensive behaviour was observed after intraplantar injection of capsaicin $(0.5 \mu \mathrm{g} / \mathrm{paw}$ in $5 \% \mathrm{EtOH} / \mathrm{PBS})$ into the left hind paw. Mice were restrained gently by covering them with a tissue and thereby, cupping it under the hand. Left paw was hold firmly with plantar side facing upwards. A 28G Hamilton needle was inserted at a shallow angle into the centre of the paw and $10 \mu \mathrm{L}$ capsaicin solution was injected slowly. The duration of nocifensive behaviours including shaking, licking, lifting, or flinching of the paw over a period of 6 min was taken.

\section{Inflammatory pain - Complete Freund's Adjuvant (CFA) injection *\#}

Persistent inflammatory pain in the hind paw was induced by intraplantar injection of CFA according to standard procedures (Minett et al., 2013).

Ten microliters of CFA suspension (cell suspension contains $1 \mathrm{mg} / \mathrm{mL}$ of heat-killed and dried Mycobacterium tuberculosis) or vehicle (sterile PBS) were injected into the hind paw as described for capsaicin injections.

* In order to minimize stress and therefore, avoid potential alterations of the proteome due to behavioural testing (Butler and Finn, 2009), CFA- and Veh-injected mice destined for tissue collection for the following calcium imaging and biochemistry experiments were not assessed for development of mechanical hypersensitivity at $24 \mathrm{~h}$. Instead, a separate cohort of mice that had been injected in parallel was tested for mechanical hypersensitivity. All of the tested mice displayed significant mechanical hypersensitivity as described previously (Minett et al., 2013) and shown in Fig. 3-1 A.

*, \# all CFA-injected animals displayed pronounced paw edema (Minett et al., 2013), which was absent in mice injected with the vehicle solution.

\section{Neuropathic pain - Spared Nerve Injury $(S N I) *$}

The spared nerve injury (SNI) paradigm was induced according to (Bourquin et al., 2006; Decosterd and Woolf, 2000). The mice received buprenorphine $(0.07 \mathrm{mg} / \mathrm{kg}$ body weight, subcutaneous (s.c.), Buprenovet巴, Bayer) $10 \mathrm{~min}$ prior to surgery. The surgery was performed under isoflurane anaesthesia (4\% induction, $1.8 \%$ maintenance). The shaved and disinfected left hind leg of the mouse was immobilized, and an incision was made at midlevel of the thigh using the femur as a landmark. The biceps femoris was opened by stretching to expose the sciatic nerve and its branches, the common peroneal, tibial and sural nerves. Using a stereomicroscope, the nerves were freed from surrounding tissue and the common peroneal and tibial nerves were ligated with a 6.0 silk suture (Braun) and transected 
together (distal to the ligation). A section of approximately $2 \mathrm{~mm}$ was removed of each nerve. Care was taken to avoid damage to the sural nerve. Sham surgery was performed using the same surgical techniques but without ligating and transecting the nerves. Muscle tissue was rearranged manually, and a thin layer of Lidocaine crème $\left(5 \%\right.$, Xylocaine ${ }^{\circledR}$, AstraZeneca) applied. Skin was closed with preferable 1 surgical micro clip. Mice received carprofen $\left(5 \mathrm{mg} / \mathrm{kg}\right.$, s.c., Rimadyl ${ }^{\circledR}$, zoetis) 5-8 h after the surgery.

Behavioural test for mechanical hypersensitivity was conducted 2 days prior and 7, 14, 21 and 26 days post surgery. The force was applied to the lateral side of the plantar surface of both hind paws. On day 28 post surgery mice were sacrificed for tissue isolation. Only IDRG from SNI mice which exhibited evident mechanical hypersensitivity at all tested time points were included for sample preparation for MS.

As for inflammatory pain, to reduce stress due to mouse behavioural testing, the tissue collection (day 28 post surgery) was separated in time from behavioural testing (day 26 post surgery).

\subsubsection{In vivo validation of candidate proteins and networks}

\section{Inhibition of mitochondrial ETC complex - Rotenone (Rot) injection *}

Inflammatory pain was induced as described above. Twenty-four hours after CFA- injection, mice were habituated for the mechanical sensitivity test $(1-2 \mathrm{~h})$. The inflamed hind paw was injected into the plantar surface with either $5 \mu \mathrm{L}$ of Veh $(0.63 \% \mathrm{DMSO} / \mathrm{PBS})$ or a suspension of $5 \mu \mathrm{L}$ of Rotenone ( $2.5 \mathrm{mM}$ in $0.63 \% \mathrm{DMSO} / \mathrm{PBS})$. Mice were acclimatized for another $15 \mathrm{~min}$ and mechanical sensitivity was assessed for up to $1 \mathrm{~h}$ after injection of Rotenone/Veh as described in 2.1.6.1. Three independent mouse cohorts were tested. In line with other studies (Joseph and Levine, 2006, 2009), Rotenone did not affect general health or motor coordination.

\section{Inhibition of protein disulfide isomerases (PDIs) - PACMA31 injection *}

Inflammatory pain was induced as described above. Twenty-four hours after CFA-injection, mice received an i.p. injection (with a maximal volume of $200 \mu \mathrm{L})$ of PACMA $31(20 \mathrm{mg} / \mathrm{kg}$ body weight) or Veh (6.9\% DMSO) in sterile PBS and were acclimatized for $2 \mathrm{~h}$ for testing of mechanical sensitivity as described. Withdrawal latencies of both hind paws were measured up to $6 \mathrm{~h}$ after injection of PACMA 31/Veh. Two independent mouse cohorts were tested. In line with another study (Xu et al., 2012), PACMA 31 did not affect general health or motor coordination.

\section{In vivo knockdown of Vtilb - Intra-sciatic nerve injection of AAV6 \#}

To downregulate Vti1b expression in DRG neurons, AAV6-Vti1b miRNA-GFP virus particles were injected into the sciatic nerve. As control AAV6-scramble miRNA-GFP virus particles were injected. The intranerval injection was performed similar to a previously published procedure (Glatzel et al., 2000) with modifications. 
The mice were injected with buprenorphine $\left(0.07 \mathrm{mg} / \mathrm{kg}\right.$, s.c., Buprenovet $\left.{ }^{\circ}\right) 10 \mathrm{~min}$ prior to surgery. Throughout the surgery mice were anesthesized via isoflurane inhalation (4\% induction, $1.8 \%$ maintenance). The left hind leg of the mouse was immobilized, hip/upper hind leg region shaved and disinfected, and an incision was made at upper level of the thigh. The sciatic nerve was exposed by dislodging the musculus biceps femoris and musculus gluteus superficialis and using retractor to keep the cavity open/tissue in place. The nerve was carefully placed under bent forceps and $0.9 \times 10^{\wedge} 8-1.3 \times 10^{\wedge} 9$ virus particles (final volume of $10 \mu \mathrm{L}(2: 1$, PBS: $20(\mathrm{w} / \mathrm{v}) \%$ mannitol) were injected with a $32 \mathrm{G}$ needle attached to a Hamilton syringe over a period of $10 \mathrm{~min}$. After repositioning of nerve and muscle tissue and Lidocaine application onto the muscle layer, the skin was closed with preferable one surgical clip. Approx. 5-8 hours post-surgery, the mice received carprofen $(5 \mathrm{mg} / \mathrm{kg}$, s.c., Rimadyl®, zoetis).

Mice undergoing virus injections were assessed for their thermal and mechanical sensitivity prior to surgery, $14 / 15 \& 22 / 23$ days after surgery. On day $24, \mathrm{CFA} / \mathrm{Veh}$ was injected to induce inflammatory pain as described above. Two independent cohorts were tested $24 \mathrm{~h}$ (day 1) after CFA-injection for thermal hypersensitivity and on day 2 for mechanical hypersensitivity as described above. Two further cohorts were tested $24 \mathrm{~h}$ (day 1) after CFAinjection for mechanical hypersensitivity and on day 2 for thermal hypersensitivity as described above. On day 4 mice were sacrificed and ipsilateral IDRG 3-5 isolated and processed for immunohistochemistry (as described above).

The cohorts destined for testing of acute pain behaviour, were injected with capsaicin on day 24 after virus-injection as described above and sacrificed the same day for tissue isolation.

\subsubsection{Mass spectrometry and bioinformatic analysis}

\subsubsection{Mass spectrometry and data analysis for project I*}

The SWATH-like DIA-MS and data analysis were performed by Roland Bruderer, Biognosys AG (Zuerich, Switzerland) as described in (Rouwette et al., 2016); in brief:

\section{Sample preparation for mass spectrometric analysis}

In total 12 samples ( 3 biological replicates per experimental group (SNI/Sham \& CFA/Veh)were prepared for MS analysis. The peptides were dissolved in $8 \mathrm{M}$ urea/0.1 $\mathrm{M}$ ammonium bicarbonate and desalted using a C18 Ultra MicroSpin Column (The Nest Group). Photospectrometry revealed that the peptide concentration was in the expected range of 1 $\mu \mathrm{g} / \mu \mathrm{L}$ for all samples. After drying, peptides were dissolved in $1 \%$ acetonitrile in water with $0.1 \%$ formic acid (LC solvent A). The HRM Calibration Kit (Biognosys) was spiked into all of the samples.Subsequently, samples were run for spectral library generation and Hyper Reaction Monitoring (HRM)-MS profiling. The peptides were separated by a linear gradient of 5-35\% solvent B in 120 min followed by $35-100 \%$ B (3\% water in acetonitrile with $0.1 \%$ formic acid) in $2 \mathrm{~min}$ and $100 \% \mathrm{~B}$ for $8 \mathrm{~min}$ (total gradient length for spectral library generation was $240 \mathrm{~min}$ ). 


\section{Spectral library generation}

For generation of the spectral library, one sample from each of the four groups was run and the results pooled. DDA LC-MS/MS acquisitions were performed on a Q Exactive mass spectrometer. The LC-MS/MS data were analysed using Maxquant 1.4 software with the following settings: protease: Trypsin/P with maximal two allowed missed cleavages; as fixed modification carbamidomethyl (C) was selected and as variable modifications oxidation (M) and acetylation (at protein $\mathrm{N}$-term) were selected; the false discovery rate was on peptide and protein level was set to $1 \%$; mass tolerance for precursor ions $20 \mathrm{ppm}$ and fragment ions $20 \mathrm{ppm}$. The spectra were searched against a mouse Uniprot FASTA database (state 05.06.2014). The generated spectral library comprised 16,971 peptide assays $(15,850$ proteotypic ones) that mateched to 3,067 proteins (2,530 of these proteins were defined by at least one proteotypic peptide).

\section{$\underline{\text { DIA-MS and data analysis }}$}

The HRM method (LC gradient of $2 \mathrm{~h}$ ) consisted of loops of 1 survey scan followed by 19 isolation windows/“SWATHs" covering 400 to $1,200 \mathrm{~m} / \mathrm{z}$. Spectronaut ${ }^{\mathrm{TM}} 5.0$ software Biognosys AG (Zuerich, Switzerland)was used to analyse the $12 \mathrm{HRM}$ measurements and the spectral library generated beforehand was used for the targeted analysis of the DIA maps. The false discovery rate (FDR) on peptide level was chosen as $1 \%$.

Proteins showing a significant differential expression in either CFA vs. Veh or SNI vs. Sham were determined by state comparison analysis using a pairwise t-test. State comparison analysis and the calculation of fold changes were performed in MSstats 2.3.2 as described in (Bruderer et al., 2015). Multiple testing correction of p-values was done with the Benjamini Hochberg (BH) method and the q-values were calculated (Storey, 2002).

The data have been uploaded to Peptide Atlas (http://www.peptideatlas.org, No. PASS00826, username: PASS00826, password: ZE5945at).

\section{Network analysis of candidate proteins}

Extensive manual literature search on Pubmed (https://www.ncbi.nlm.nih.gov/pubmed/) was done along with querying the Pain Networks database (Perkins et al., 2013) to find out whether the 141 regulated candidates have previously been reported to be related to pain states in mice/rats and humans. To determine the novel pain-related proteins in our study, we excluded all genes/proteins (I) with an involvement in nociception and pain that has been reported previously, (II) which are pain-related genes in the Pain Networks database, or (III) members of the mitochondrial electron transport chain (ETC) given that inhibitors of mitochondrial ETC modulate pain (Ferrari et al., 2011; Joseph and Levine, 2009). Differentially regulated proteins were annotated with Gene ontology (GO) terms (Ashburner et al., 2000) and enriched GO terms were determined with the DAVID functional annotation tool (Huang et al., 2008a). Only significantly enriched GO terms with BH corrected p-values $<0$. are reported. The Search Tool for the Retrieval of Interacting Genes/Proteins (STRING) was used to search for/display direct and indirect interactions among all significantly regulated proteins in each pain model (Szklarczyk et al., 2015). STRING settings: 
Confidence view; confidence level 0.4; clustering algorithm MCL set to 2. In Fig. 3.3 only with at least one connection are displayed and therefore, not all proteins showing a significant regulation are included in the displayed networks .

\subsubsection{Mass spectrometry and data analysis of TRPV1-CoIP samples \#}

\section{MS acquisition and raw data processing}

The elutes of the CoIPs were loaded on Prime Tris/glycine 8-16\% gradient gels (Serva) and proteins separated by electrophoresis. After visualization of the proteins by Coomassie staining, each of the gel lanes was cut into 24 pieces of equal size that were subjected to automated in-gel digestion with trypsin (Schmidt et al. 2013). With help of a vacuum centrifuge, the digested peptides were dried and re-dissolved in $0.1 \%$ trifluoro acetic acid. To allow for absolute quantification later on, a standard digest of yeast enolase 1 (2.5 $\mathrm{fmol} / \mu \mathrm{L}$, Waters Corporation) was added (Silva et al., 2006). Peptides were separated by nanoscale reversed-phase UPLC (Ultra Performance Liquid chromatography, Waters Corporation) and subsequently subjected to $\mathrm{UDMS}^{\mathrm{E}}$ (Ultra-definition $\mathrm{MS}^{\mathrm{E}}$, a type of DIAMS; (Distler et al., 2014, 2016)) analysis on a Waters Synapt G2-S quadrupole- time of flight (Q-TOF) with ion mobility shift (IMS) option. Acquisition of positive ions in the $\mathrm{m} / \mathrm{z}$ range of 50-2000 and a resolution of $\geq 20,000$ FWHM was done in the IMS-enhanced data independent acquisition mode (Silva et al., 2006) with drift time-specific collision energies (Distler et al., 2014, 2016).

The initial signal processing of continuum LC-IMS-MS data was done with ProteinLynx Global Server v.3.0.2 (PLGS, Waters). For peptide and protein identification a customized database was generated by adding sequences of porcine trypsin and yeast enolase 1 to the UniProtKB/Swiss-Prot murine proteome (release 2016/11;16839 entries), as well as expanding it with the reversed sequences of all entries to allow for determination of the false discovery rate (FDR). Ion Mass tolerances of precursor and fragment ions were typically below 5pm and 100ppm (root mean square), respectively, as determined automatically by PLGS v.3.0.2. Trypsin was set as digestion enzyme and 1 missed cleavage allowed for peptide identification. Methionine oxidation was set as variable modification and the carbamidomethylation of cysteine residues as fixed modification. At least two fragments per peptide, five fragments per protein, and one peptide per protein were the minimal requirements for ion matching. An FDR of $1 \%$ was set as threshold for protein identification.

For data post-processing and the label-free quantification, the open source software ISOQuant (http://www.isoquant.net; (Kuharev et al., 2015)) was used. This included retention time alignment, exact mass and retention time (EMRT) and ion mobility clustering, data normalization, isoform/homology filtering, and the absolute in-sample amount was determined by a modified TOP3 method (Distler et al., 2014, 2016; Kuharev et al., 2015).

The stringency for reporting a protein was increased by only considering Peptides identified already in the first PLGS database search (described above) that consisted of at least 6 amino acids and had a score of $\geq 5.5$. The FDR level was set to $1 \%$ at both peptide and protein level. Only for proteins with a minimum of 2 peptides, absolute protein quantities were estimated 
with the TOP3 method. The LC-IMS-MS datasets from each of the 24 gel pieces of one gel lane were merged into one file. To increase the overlap of peptide identifications across the 3 biological replicates of one group, cross-annotation of feature clusters using the PLGS peptide information of matching features was enabled, but only within the 3 replicates of one group, not across the 3 different groups.

The mass spectrometry acquisition as well as the data post processing and quantification as described above was done by Olaf Jahn (Proteomics group, MPIem).

\section{Data analysis for defining specific interactors of TRPVI}

Further data processing was done with Microsoft excel and the freely available Perseus software (v1.5.6.0.; (Tyanova et al., 2016)):

The output list generated by ISOQuant was filtered for proteins exhibiting a PLGS score of at least 800 in one of the groups, yielding 1089 proteins. The ratio of the TRPV1 amount in a CFA sample to the TRPV1 amount in the Veh sample that was run in parallel, was taken to normalize protein abundances in CFA. Then data was uploaded to Perseus and after log2transformation of the abundance values (in amol) of all 9 samples, they were plotted against each other and the Pearson correlation coefficient was calculated.

The triplicate samples were grouped (CFA, Veh and KO) and filtered for having at least $3 / 3$ identifications in one of the three groups.

To enable statistical analysis, missing values were imputated. Two different methods of imputation were used (I) with a constant value of 5.41 ( $\log 2$ value of the lowest detected value over all runs, shifted down for one order of magnitude) and (II) random values taken from a normal distribution simulating low abundance values around the limit of detection of the mass spectrometer. The mean and standard deviation of the distribution of the protein abundancies from each group were calculated and a new distribution, respectively, with a width of 0.3 standard deviations and a downward shift of 1.8 standard deviations was created (Hanack et al., 2015; Hubner et al., 2010).

To assess significantly enriched proteins in CFA/Veh vs. the $\mathrm{KO}$ control, group-wide comparison was done using a modified Student's t-test (Tusher et al., 2001). The s0 parameter was set to 1.58 (i.e. proteins need to exhibit a minimal fold change of 3) and the FDR (calculated by a permutation-based strategy) to 0.05 . The outcome was plotted in a volcano plot with a cut-off curve representing a minimal fold change of 3 ( $\mathrm{s} 0=1.58)$ and an FDR of 0.05 .

Subsequently, the fold change of significantly regulated proteins in CFA vs. Veh were calculated (only proteins significantly enriched in both or one of the groups compared to KO were considered). A threshold of a $\pm 20 \%$ alteration between the groups was set to define pain-specific interactions. 


\section{Network analysis of candidate interactors}

The Database for Annotation, Visualization and Integrated Discovery (DAVID v6.8, https://david.ncifcrf.gov/home.jsp) was used to determine Gene Ontology terms of proteins significantly enriched compared to KO control. Furthermore, using the Functional Clustering tool (pre-set standard options and medium classification stringency) enriched annotation clusters were determined.

The STRING (Search Tool for the Retrieval of Interacting Genes/Proteins, https://stringdb.org/) database was searched and used to visualize previously known and predicted interactions among significantly regulated proteins in CFA and Veh, respectively, STRING settings: Confidence view; confidence level 0.7; interaction sources: experiments and databases; clustering algorithm MCL set to 3. 


\subsection{Material}

Table 2-1: Details of material used in this study

\begin{tabular}{|c|c|c|}
\hline Product & Company & Cat \# \\
\hline Acetone Rotisolv® HPLC & Roth & 7328.1 \\
\hline Agarose & Roth & 3810.4 \\
\hline Allyl isothiocyanate (Mustard oil, MO), 95\% & Sigma-Aldrich & $377430-100 \mathrm{G}$ \\
\hline BAPTA $\times 4 \mathrm{~K}+$ & Life Technologies & B1204 \\
\hline Benchtop 1kb DNA Ladder & Promega & G754A \\
\hline BlueJuice $^{\mathrm{TM}}$ Gel Loading Buffer, 10X & Life Technologies & 10816015 \\
\hline BSA (Bovine serum albumin) & Sigma-Aldrich & A7030-50G \\
\hline $\mathrm{CaCl}_{2}$ & Roth & CN92.1 \\
\hline Capsaicin & Sigma-Aldrich & M2028-50MG \\
\hline CFA (Complete Freund's Adjuvant) & Sigma-Aldrich & F5881-10ML \\
\hline Collagenase, Type IV & Life Technologies & 13028014 \\
\hline $\begin{array}{l}\text { cOmplete }^{\mathrm{TM}} \text { ULTRA Tablets, } \\
\text { Mini, EASYpack Protease Inhibitor Cocktail }\end{array}$ & Roche & 5892970001 \\
\hline D-Mannitol & Sigma-Aldrich & M4125-100G \\
\hline n-Dodecyl- $\beta$-D-maltoside & Roth & CN26.3 \\
\hline $\begin{array}{l}\text { DMEM (Dulbecco's modified eagle medium), } \\
\text { high glucose, GlutaMAX(TM), pyruvate }\end{array}$ & Life Technologies & $31966-021$ \\
\hline DMEM /Ham's F-12, GlutaMAX & Life Technologies & $31331-028$ \\
\hline $\begin{array}{l}\text { DMSO (dimethyl sulfoxide), anhydrous, } \\
\geq 99.9 \%\end{array}$ & Sigma-Aldrich & 276855 \\
\hline Donkey serum & Dianova & $017-000-121$ \\
\hline $\begin{array}{l}\text { DPBS (Dulbecco's phosphate-buffered saline), } \\
\text { no calcium, no magnesium, } 1 \times\end{array}$ & Life Technologies & $14190-136$ \\
\hline $\begin{array}{l}\text { DTT (Dl- Dithiothretol) solution ( 1 M in } \\
\left.\mathrm{H}_{2} \mathrm{O}\right)\end{array}$ & Sigma-Aldrich & 43816-10ML \\
\hline Duolink® In Situ Detection Reagents Orange & Sigma-Aldrich & DUO92007 \\
\hline Dynabeads Protein $\mathrm{G}$ & Life Technologies & $10004 \mathrm{D}$ \\
\hline Dynasore Monohydrate & Sigma-Aldrich & D7693-5MG \\
\hline EGTA (ethylene glycol tetraacetic acid) & Roth & 3054.2 \\
\hline FBS (Fetal bovine serum) & Life Technologies & $10270-106$ \\
\hline Fibronectin from bovine plasma & Sigma-Aldrich & F4759-1MG \\
\hline FuGENE ® HD transfection reagent & Promega & E2311 \\
\hline $\begin{array}{l}\text { Fura-2, AM (acetoxymethyl ester), cell } \\
\text { permeant }\end{array}$ & Life Technologies & F1221 \\
\hline alpha-D(+)-Glucose Monohydrat & Roth & 6887.1 \\
\hline Glycerol & Roth & 7530.1 \\
\hline Goat serum & Dianova & $005-000-121$ \\
\hline
\end{tabular}




\begin{tabular}{|c|c|c|}
\hline $\begin{array}{l}\text { Growth factors (NGF, GDNF, BDNF, NT-3, } \\
\text { NT-4) }\end{array}$ & R\&D Systems & $\begin{array}{c}\text { 212-GD-010, } \\
\text { 268-N4-005, } \\
267-N 3-005, \\
248-\mathrm{BD}-005, \\
256-\mathrm{GF}-100\end{array}$ \\
\hline $\begin{array}{l}\text { HBSS (Hanks' Balanced Salt Solution), } \\
\text { calcium, magnesium, no phenol red, 10x }\end{array}$ & Life Technologies & 14065049 \\
\hline $\begin{array}{l}\text { HEPES (4-(2-hydroxyethyl)-1- } \\
\text { piperazineethanesulfonic acid), 1M }\end{array}$ & Life Technologies & $15630-056$ \\
\hline Horse serum & Life Technologies & $16050-122$ \\
\hline $\mathrm{KCl}$ & Roth & 6781.3 \\
\hline Laminin Mouse Protein, Natural & Life Technologies & $23017-015$ \\
\hline Library Efficiency DH5 $\alpha$ Competent Cells & ThermoFisher Scientific & $18263-012$ \\
\hline $\mathrm{MgCl}_{2}$ & Roth & KK36.1 \\
\hline Milk powder & Roth & T145.2 \\
\hline $\mathrm{Na}_{2} \mathrm{CO}_{3}$ & Roth & P028.1 \\
\hline $\mathrm{NaCl}$ & Roth & P029.3 \\
\hline $\mathrm{NaOH}$ (sodium hydroxide), $50 \%$ & Roth & 8655.1 \\
\hline NMDG( N-Methyl-D-glucamine) & Sigma-Aldrich & M-2004 \\
\hline$\left(\mathrm{NH}_{4}\right) \mathrm{HCO}_{3}$ & Roth & T871.1 \\
\hline Novex Sharp Pre-stained Protein Ladder & Life technologies & LC5800 \\
\hline NucleoSpin $®$ Gel and PCR Clean-up & Macherey Nagel & 740609 \\
\hline NucleoSpin® RNA XS extraction kit & Macherey Nagel & 740902 \\
\hline iBlot® Transfer Stack, PVDF & Life Technologies & IB401001 \\
\hline NuPAGE® Antioxidant & Life Technologies & NP0005 \\
\hline NuPAGE® Bis-Tris 4-12\% gradient gels & Life Technologies & NP0336BOX \\
\hline NuPAGE® LDS Sample Buffer, $4 \times$ & Life Technologies & NP0007 \\
\hline NuPAGE® MOPS SDS running buffer, 20x & Life Technologies & NP 0001 \\
\hline $\begin{array}{l}\text { NuPAGE® Sample Reducing Agent, } \\
10 \times(500 \mathrm{mM} \text { DTT })\end{array}$ & Life Technologies & NP0009 \\
\hline $\begin{array}{l}\text { Opti-MEMTM (Minimal Essential Medium)I } \\
\text { Reduced Serum Medium }\end{array}$ & Life Technologies & $31985-062$ \\
\hline $\begin{array}{l}\text { P3 Primary Cell 4D-Nucleofector® X Kit S } \\
(32 \text { RCT) }\end{array}$ & Lonza & V4XP-3032 \\
\hline $\begin{array}{l}\text { PACMA31 (Protein Disulfide Isomerase } \\
\text { Inhibitor III) }\end{array}$ & Merck- & $539225-25 \mathrm{MG}$ \\
\hline Papain, $20 \mathrm{U} / \mathrm{mL}$ & Worthington & LK003178 \\
\hline PBS, pH 7.4, 10× & Life Technologies & 70011-051 \\
\hline Penicillin-Streptomycin $(5,000 \mathrm{U} / \mathrm{mL})$ & Life Technologies & $15070-063$ \\
\hline $\begin{array}{l}\text { PFA, Paraformaldehyde Aqueous Solution, } \\
\text { EM Grade, } 16 \%\end{array}$ & Science services & E15710 \\
\hline Poly-D-Lysine solution, $1.0 \mathrm{mg} / \mathrm{mL}$ & Millipore & A-003-E \\
\hline Power SYBR green PCR master mix & Life Technologies & 4367659 \\
\hline PureLink ${ }^{\circledR}$ Quick Plasmid Miniprep Kits & Life Technologies & K210010 \\
\hline QuantiTect Reverse Transcription Kit & Qiagen & 205313 \\
\hline RapiGest ${ }^{\mathrm{TM}} \mathrm{SF}$ & Waters & 186001861 \\
\hline
\end{tabular}




\begin{tabular}{|l|l|c|}
\hline Rotenone & Sigma-Aldrich & R8875 \\
\hline $\begin{array}{l}\text { RPMI (Roswell Park Memorial Institute) } 1640 \\
\text { medium }\end{array}$ & Life Technologies & $31870-017$ \\
\hline Slowfade Gold antifade reagent with DAPI & Life Technologies & S36938 \\
\hline Sucrose & Roth & 4621.1 \\
\hline SURE® 2 Supercompetent Cells & Agilent Technologies & 200152 \\
\hline Tris/Glycine-SDS Sample Buffer, 2× & SERVA & 42527 \\
\hline $\begin{array}{l}\text { TissueTek O.C.T. (optimal cutting } \\
\text { temperature) compound }\end{array}$ & Sakura & 4583 \\
\hline Tris-HCl & Roth & 9090.3 \\
\hline Triton X-100 & Roth & 3051.3 \\
\hline TrypLETM Express Enzyme (1×), phenol red & Life Technologies & 12605010 \\
\hline Tween 20 & Roth & 9127.1 \\
\hline Water For Injection (WFI) for Cell Culture & Life Technologies & A1287301 \\
\hline
\end{tabular}

Table 2-2: Details of antibodies across experiments

\begin{tabular}{|c|c|c|c|c|c|c|}
\hline Antibody & IHC & Live & CoIP & ICC/PLA & WB & Details \\
\hline Goat $\alpha$-TRPV 1 & $1: 200$ & l & $12 \mu \mathrm{g}$ & $\begin{array}{c}1: 100 / \\
1: 250-400\end{array}$ & $1: 100$ & $\begin{array}{l}\text { Santa Cruz, } \\
\text { SC-12498 }\end{array}$ \\
\hline Rabbit $\alpha$-TRPV1 & l & I & $12 \mu \mathrm{g}$ & I & $1: 200$ & $\begin{array}{l}\text { Alomone, } \\
\text { ACC-030 }\end{array}$ \\
\hline Rabbit $\alpha-V$ ti $1 b$ & $1: 200$ & I & I & $\begin{array}{l}1: 750 / \\
1: 750\end{array}$ & $1: 750$ & $\begin{array}{l}\text { Synaptic } \\
\text { Systems, } \\
164002\end{array}$ \\
\hline Mouse $\alpha-c-M y c$ & I & I & I & $\begin{array}{l}1: 100 / \\
1: 750\end{array}$ & $1: 100$ & $\begin{array}{l}\text { Santa Cruz, } \\
\text { SC-47694 }\end{array}$ \\
\hline $\begin{array}{l}\text { Rabbit } \alpha- \\
\text { AnnexinA2 }\end{array}$ & l & I & I & l & $1: 100$ & $\begin{array}{l}\text { Abcam, } \\
\text { ab41803 }\end{array}$ \\
\hline $\begin{array}{l}\text { Rabbit } \alpha \text {-TRPV1 } \\
\text { extracellular }\end{array}$ & l & $1: 50$ & / & I & I & $\begin{array}{l}\text { Alomone, } \\
\text { ACC-029 }\end{array}$ \\
\hline Chicken $\alpha$-GFP & $1: 500$ & I & I & / & I & $\begin{array}{l}\text { Life } \\
\text { Technologies, } \\
\text { A10262 }\end{array}$ \\
\hline Mouse $\alpha$ - $\beta$-actin & $1: 500$ & I & I & / & l & $\begin{array}{l}\text { Sigma, } \\
\text { A1978 }\end{array}$ \\
\hline $\begin{array}{l}\text { Rabbit } \\
\alpha-\mathrm{Na}+\mathrm{K}+- \\
\text { ATPase } \alpha 2\end{array}$ & $1: 100$ & I & I & / & I & $\begin{array}{l}\text { Millipore, } \\
07-67\end{array}$ \\
\hline Rat $\alpha$-Lamp-1 & I & I & I & $1: 500$ & I & $\begin{array}{l}\text { BD } \\
\text { Pharmingen, } \\
553792\end{array}$ \\
\hline $\begin{array}{l}\text { Donkey } \alpha \text {-Rabbit } \\
\text { AF488 }\end{array}$ & I & I & I & $1: 250$ & I & $\begin{array}{l}\text { Life } \\
\text { Technologies, } \\
\text { A21206 }\end{array}$ \\
\hline
\end{tabular}




\begin{tabular}{|c|c|c|c|c|c|c|}
\hline $\begin{array}{l}\text { Donkey } \alpha \text {-Rabbit } \\
\text { AF546 }\end{array}$ & I & $1: 200$ & I & $1: 250$ & I & $\begin{array}{l}\text { Life } \\
\text { Technologies, } \\
\text { A10040 }\end{array}$ \\
\hline $\begin{array}{l}\text { Donkey } \alpha \text {-Rabbit } \\
\text { AF647 }\end{array}$ & $1: 250$ & $1: 200$ & I & $1: 250$ & I & $\begin{array}{l}\text { Life } \\
\text { Technologies, } \\
\text { A31573 }\end{array}$ \\
\hline $\begin{array}{l}\text { Donkey } \alpha \text {-chicken } \\
\text { AF488 }\end{array}$ & $1: 500$ & I & I & 1 & I & $\begin{array}{l}\text { Biotium, } \\
20166\end{array}$ \\
\hline $\begin{array}{l}\text { Donkey } \alpha \text {-Goat } \\
\text { AF546 }\end{array}$ & $1: 250$ & I & I & $1: 250$ & I & $\begin{array}{l}\text { Life } \\
\text { Technologies, } \\
\text { A11056 }\end{array}$ \\
\hline $\begin{array}{l}\text { Donkey } \alpha \text {-Mouse } \\
\text { AF647 }\end{array}$ & I & I & I & $1: 250$ & I & $\begin{array}{l}\text { Life } \\
\text { Technologies, } \\
\text { A31571 }\end{array}$ \\
\hline $\begin{array}{l}\text { Donkey } \alpha \text {-Mouse } \\
\text { AF680 }\end{array}$ & I & I & I & I & $1: 8000$ & $\begin{array}{l}\text { Life } \\
\text { Technologies, } \\
\text { A10038 }\end{array}$ \\
\hline $\begin{array}{l}\text { Donkey } \alpha \text {-Rabbit } \\
\text { AF680 }\end{array}$ & I & I & I & I & 1:8000 & $\begin{array}{l}\text { Life } \\
\text { Technologies, } \\
\text { A10043 }\end{array}$ \\
\hline $\begin{array}{l}\text { Donkey } \alpha \text {-Goat } \\
\text { AF680 }\end{array}$ & I & I & I & I & $1: 8000$ & $\begin{array}{l}\text { Life } \\
\text { Technologies, } \\
\text { A21084 }\end{array}$ \\
\hline $\begin{array}{l}\text { Donkey } \alpha \text {-Rat } \\
\text { DyLight } 550\end{array}$ & I & I & I & $1: 250$ & I & $\begin{array}{l}\text { Life } \\
\text { technologies, } \\
\text { SA510027 }\end{array}$ \\
\hline $\begin{array}{l}\text { Duolink® In Situ } \\
\text { PLA® Probe Anti- } \\
\text { rabbit MINUS }\end{array}$ & I & I & I & $1: 10$ & I & $\begin{array}{l}\text { Sigma, } \\
\text { DUO92005 }\end{array}$ \\
\hline $\begin{array}{l}\text { Duolink® In Situ } \\
\text { PLA® Probe Anti- } \\
\text { Mouse PLUS }\end{array}$ & I & I & I & $1: 10$ & I & $\begin{array}{l}\text { Sigma, } \\
\text { DUO92001 }\end{array}$ \\
\hline $\begin{array}{l}\text { Duolink® In Situ } \\
\text { PLA® Probe Anti- } \\
\text { goat PLUS }\end{array}$ & I & I & I & $1: 10$ & I & $\begin{array}{l}\text { Sigma, } \\
\text { DUO92003 }\end{array}$ \\
\hline
\end{tabular}

AF, Alexa Fluor; CoIP, Co-immunoprecipitation; Live, Live labelling; ICC, Immunocytochemistry; IHC, immunohistochemistry; PLA, Proximity Ligation Assay 
Table 2-3: Details of DNA plasmids used in this study

\begin{tabular}{|l|l|}
\hline \multicolumn{1}{|c|}{ Construct } & \multicolumn{1}{c|}{ Details } \\
\hline TRPV1-YFP & $\begin{array}{l}\text { pcDNA5/FRT plasmid, YFP tagged, rat TRPV1, kind gift } \\
\text { from Ardem Patapoutian }\end{array}$ \\
\hline Vti1b-myc-DDK & $\begin{array}{l}\text { pCMV6-Entry plasmid, Myc and DDK(Flag)-tagged, mouse } \\
\text { Vti1b, Origene }\end{array}$ \\
\hline pCMV-Sport6 & Empty Vector, kind gift from Ardem Patapoutian \\
\hline Vti1b-miRNA 231 & pUC57-Entry plasmid, miRNA $\alpha$ mouse Vti1b, GeneScript \\
\hline $\begin{array}{l}\text { AAV6-miRNA 231- } \\
\text { GFP }\end{array}$ & Self-generated, see section 2.1.3.1 \\
\hline $\begin{array}{l}\text { AAV6-control } \\
\text { miRNA-GFP }\end{array}$ & See section 2.1.3.1, kind gift from Sebastian Kügler \\
\hline
\end{tabular}

Table 2-4: Details of siRNA used in this study

\begin{tabular}{|l|l|}
\hline \multicolumn{1}{|c|}{ siRNA } & \multicolumn{1}{c|}{ Details } \\
\hline Control siRNA & $\begin{array}{l}\text { AllStar Negative Control siRNA, sequence: propriety } \\
\text { information, Qiagen, \#1027280 }\end{array}$ \\
\hline Vti1b siRNA: & $\begin{array}{l}\text { FlexiTube GeneSolution targeting mouse Vti1b, Qiagen, } \\
\text { \#GS53612 (mix of 4 different siRNAs): }\end{array}$ \\
Mm_Vti1b_2 & AACGAGCATTTGAATCGACTA \\
Mm_Vti1b_3 & CGCGGCCTCCTTGAAGACTTA \\
Mm_Vti1b_5 & CAAGACCAGTATGATATACTA \\
Mm_Vti1b_6 & CAGCATCTTACTCTGAATGAA \\
\hline
\end{tabular}

Mm, Mus musculus

Table 2-5: Details of qPCR-primers used in this study

\begin{tabular}{|c|c|}
\hline Primer name & Primer sequence (5'-3') \\
\hline Mm_GAPDH_forward & CAATGAATACGGCTACAGCAAC \\
\hline Mm_GAPDH_reverse & TTACTCCTTGGAGGCCATGT \\
\hline $\mathrm{Mm}_{-} \beta$-actin_forward & GATCAAGATCATTGCTCCTCCTG \\
\hline $\mathrm{Mm}_{-} \beta$-actin_reverse & CAGCTCAGTAACAGTCCGCC \\
\hline Mm_Vti1b_forward & TCTTCGCTCAATGTCCAGAA \\
\hline Mm_Vtilb_reverse & TACACCAGACCGACCAGGAT \\
\hline
\end{tabular}

Mm, Mus musculus 


\section{Results}

\subsection{Insights into chronic pain by standardized DRG proteome profiling}

This research was originally published in Molecular \& Cellular Proteomics. Rouwette, Sondermann et al.. Standardized profiling of the membrane-enriched proteome of mouse dorsal root ganglia (DRG) provides novel insights into chronic pain. Mol Cell Proteomics. 2015; 15(6), 2152-2168. (C) the American Society for Biochemistry and Molecular Biology. Thus, most of this section is based on Rouwette et al., 2016, with emphasis on the work I was significantly involved in.

Effective treatment of chronic pain has not been achieved so far and faces major challenges. This is mainly due to the fact that (I) the molecular pathomechanisms are unknown for the variety of chronic pain syndromes and (II) as a consequence, current analgesics target molecules with key physiological function throughout our body, thus, are often accompanied by distressing side effects for the patients. A better understanding of the molecular events underlying pathological pain is desirable to develop better therapeutics. In fact, compounds targeting proteins highly enriched in the nociceptors - as they exert their function in the first line of noxious stimulus detection and accordingly critically shape the transmission of pain messages to the brain - represent a major goal in pain research. In order to identify proteins with a putative role in the chronification of pain, we used an unbiased MS-based approach - combining mouse behaviour with cutting-edge SWATH-like DIA-MS - to characterize the differential regulation of DRG-enriched proteins in different pain states in mice. Fig. 3.1 gives an overview about our experimental workflow. 


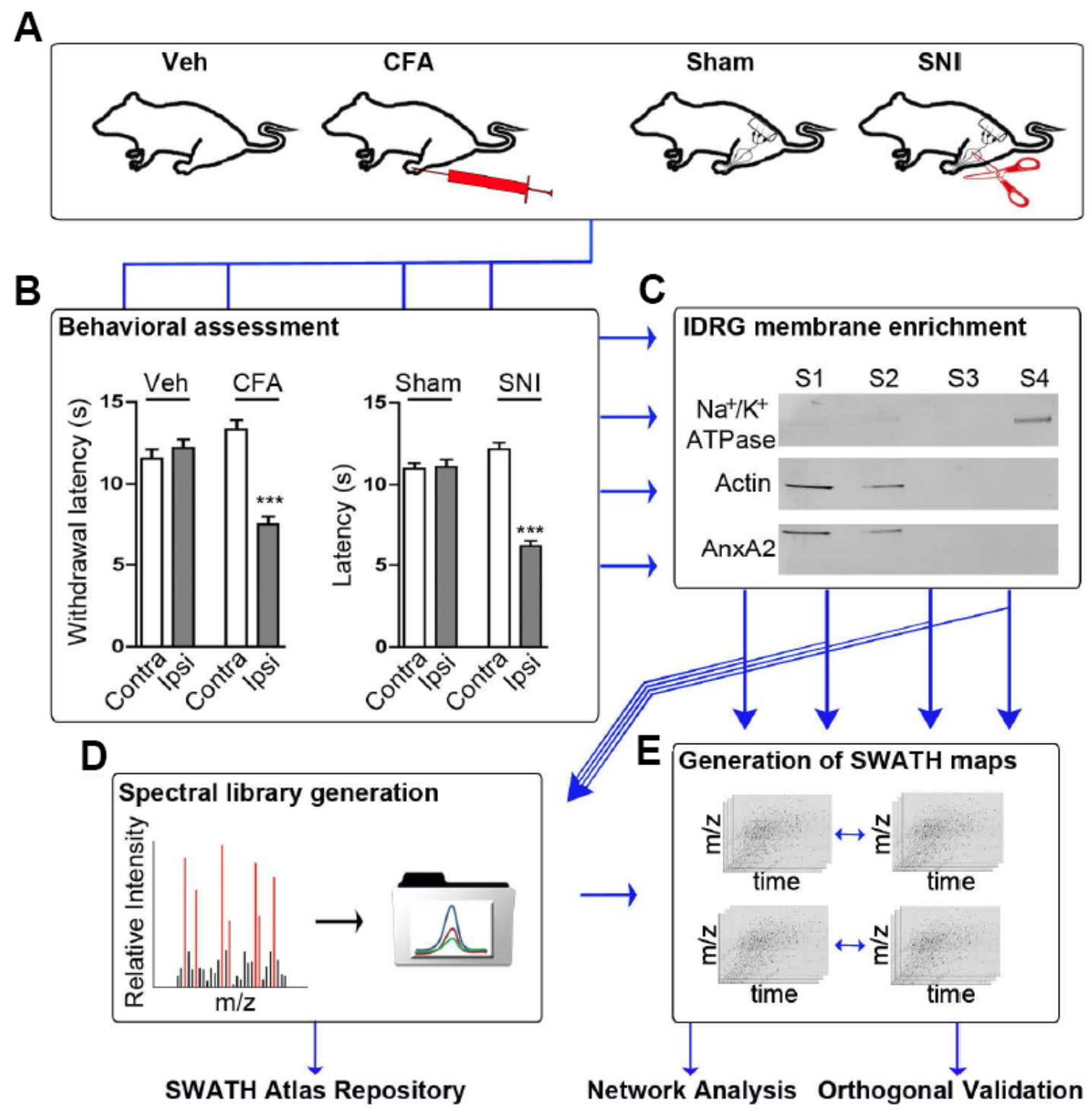

Figure 3.1: Scheme of the experimental workflow

(A) Illustration of the 4 behavioural conditions used in this study (from left to right): Injection of Veh (vehicle solution) vs. injection of CFA into one hind paw, sham surgery (exposed but untouched sciatic nerve) vs. SNI (exposed and partial transected sciatic nerve). (B) Mice in the CFA and SNI groups show a decrease in paw withdrawal latency of the ipsilateral, i.e. treated site, indicating mechanical hypersensitivity. Paw withdrawal latencies of contralateral (contra) hind paws and in the Veh or Sham group did not change, $* * * \mathrm{p}<0.001, \mathrm{~N}=21-24$ mice/condition, $\mathrm{n}=3$ independent cohorts, one-way ANOVA followed by Bonferroni's multiple comparison tests. (C) Representative immunoblot of subfractions of our biochemical membrane enrichment (S1-4). The membrane marker $\mathrm{Na}^{+} / \mathrm{K}^{+}$-ATPase showed enrichment in the membraneenriched fraction (S4), whereas cytosolic markers $\beta$-actin and annexinA2 (AnxA2) were decreased. An opposite result was obtained in the cytosolic-enriched fractions ( $\mathrm{S} 1$ and $\mathrm{S} 2, \mathrm{n}=3$ independent preparations). (D, E) Schematic representation of spectral library generation and DIA-MS profiling and follow-up experiments. This research was originally published in Molecular \& Cellular Proteomics. Rouwette, Sondermann et al.. Standardized profiling of the membrane- enriched proteome of mouse dorsal root ganglia (DRG) provides novel insights into chronic pain. Mol Cell Proteomics. 2015; 15(6), 2152-2168. (C) the American Society for Biochemistry and Molecular Biology. 


\subsubsection{Animal models of chronic pain and sample preparation}

As experimental models for chronic pain, we chose to investigate two established models of pathological pain with different aetiology (Minett et al., 2013): On the one hand, we induced persistent inflammatory pain by injecting complete Freund's adjuvant (CFA; and saline as vehicle (Veh) control) into the hind paw of mice. This is accompanied by development of profound hypersensitivity of the injected paw starting within few hours after the injection, and reaching its peak at $24 \mathrm{~h}$ (Fehrenbacher et al., 2012). Injection of CFA at different sites is used to model inflammatory pain conditions that might occur in clinical settings of tendonitis and rheumatoid arthritis (Gregory et al., 2013). On the other hand, we subjected mice to the spared nerve injury (SNI; sham-operated mice (Sham) as control) as a model for peripheral neuropathic pain (Decosterd and Woolf, 2000). In the SNI surgery, the sural, common peroneal, and tibial nerve - the terminal branches of the sciatic nerve innervating the hind paw - are exposed and subsequently the common peroneal, and tibial nerve are transected, whereas the sural one is left untouched. Within 4 days of surgery and lasting for several months, the mice develop hypersensitivity to mechanical stimulation in the plantar region of the hind paw that is innervated by the sural nerve. Importantly, mechanical allodynia is a common manifestation of several neuropathic pain syndromes in humans (Woolf and Mannion, 1999).

Accordingly, we assessed the development mechanical allodynia in the mice subjected to either SNI/Sham-surgery or CFA/Veh-injection (Fig. 3.1 B). Only tissue from mice that presented profound hypersensitivity were included in the study. Mice were sacrificed $24 \mathrm{~h}$ after injection (CFA) or 28 days later (SNI) for the isolation of the ipsilateral lumbar DRG (i.e. DRG innervating the affected hind paw). At these time points mice presented with profound mechanical hypersensitivity, which is in accordance with previous publications (Decosterd and Woolf, 2000; Minett et al., 2013).

Transmembrane proteins together with membrane associated proteins (hereafter summarized as "membrane proteins") expressed by the nociceptors located in the DRG, play an essential role in the detection and transfer of pain messages to the brain, therefore, we were especially interested in capturing their regulation. Yet, their identification and quantification by MS is hampered by their high resistance to digestion and extraction due to their inherent hydrophobicity, low solubility, and an overall low expression (Helbig et al., 2010).

We aimed to increase probability of MS-detection by preparing membrane-enriched fractions of DRG tissue lysates by biochemical subcellular fractionation. As depicted in Fig. 3.1 C, immuno-detection of $\mathrm{Na}^{+} / \mathrm{K}^{+}$-ATPase, a ubiquitous transmembrane protein, showed a clear enrichment in the membrane fraction (S4) compared to cytosolic fractions of our biochemical fractionation protocol. Conversely, cytosolic proteins like $\beta$-actin and annexin A2 (AnxA2) were not detectable in S4, indicating a successful depletion of cytosolic proteins in the membrane-enriched fraction (Fig. 3.1 C).

\subsubsection{Generation of a membrane-enriched spectral library of mouse DRG neurons}

Since the reliable identification and quantification of 1000s of proteins across several complex samples using SWATH-like DIA-MS relies on the a priori knowledge of the chromatographic and mass spectrometric signatures of peptides belonging to these proteins, 
we first created our own sample-specific spectral library. To this end, one replicate of each condition (CFA, Veh, SNI, and Sham) was analysed by shotgun MS in the DDA mode. The resulting information/peptide signatures were used to build up a non-redundant spectral library (see supplemental Table S1 in (Rouwette et al., 2016)), containing 16,971 peptide signatures $(15,850$ were proteotypic) belonging to 3,067 proteins (2,530 were defined by at least 1 proteotypic peptide).

Using DAVID (Database for Annotation, Visualization and Integrated Discovery v6.8; (Huang et al., 2008a)), we investigated the cellular compartments or molecular functions that were represented in our spectral library. Proteins were identified from a range of subcellular compartments and with the expected clear enrichment for membrane-associated proteins (see supplemental Fig. S2 in (Rouwette et al., 2016)). Among these were ion channels linked to somatosensation, for instance, TRPA1 and Piezo2 that were not detected in a previous MS study of DRG membrane-enriched tissue samples (Xiong et al., 2009).

Comparing our spectral library to previously reported proteomes of SC (containing the central terminals of DRG neurons), DRG, sciatic nerve and neuromas, reveals that it is the largest compendium of mouse DRG proteins reported to date (Huang et al., 2008b; Melemedjian et al., 2013; Niederberger and Geisslinger, 2008; Xiong et al., 2009; Zou et al., 2012). The generated data is stored in PeptideAtlas (http://www.peptideatlas.org/; (Deutsch et al., 2008)), a publicly accessible platform for sharing high-throughput proteomics data, and can be used for any DIA-MS experiment in any murine tissue of interest.

\subsubsection{DIA-MS reveals the differential regulation of proteins in rodent models of inflammatory and neuropathic pain}

To assess the differential regulation of proteins between pain and control condition, we submitted 3 biological replicates of each pain (SNI \& CFA) and control (Sham \& Veh) condition to SWATH-like DIA-MS, and for the targeted search of the acquired peptide signatures our spectral library was used. Across all replicates and samples more than 14,500 peptides were identified corresponding to the same set of 2,526 proteins (Fig. 3.2 A). Moreover, in all CFA- and Veh-replicates an overlap of 2,581 proteins and an overlap of 2,600 proteins in all SNI and Sham replicates were profiled, showing the high reproducibility of our workflow. Visualization of the protein abundances over all conditions and replicates in a heat map and volcano plot offers an overview of the global changes in abundance and reveals up- and downregulations of particular proteins (Fig. 3.2). Group-wise comparison using Student's t-test and the q-value method to control the overall false discovery rate (Storey, 2002) revealed 64 significantly regulated proteins in inflammatory pain, and 77 proteins in neuropathic pain, constituting in total about $3 \%$ of all quantified proteins (Fig. 3.2 A; see supplemental Table S3 in (Rouwette et al., 2016) for complete dataset). Comparison of the significantly regulated proteins in both pain paradigms found only 12 commonly regulated ones, proposing that the changes happening are rather specific to a pain model (Fig. 3.2 A; see supplemental Table S4 in (Rouwette et al., 2016) for list of overlapping proteins).

To gain more insight into the kind of proteins that were regulated in the two different pain paradigms, we performed GO (gene ontology)-analysis. As shown in Fig. 3.2 B (and also 
supplemental Fig. S6 in (Rouwette et al., 2016)), we found that the proteins affected, belong to a broad range of different and partially overlapping cellular locations as well as diverse functions.

Looking for previously annotated links of the significantly regulated protein to painful pathologies (see section 2.1.7.1 for details on search), we found that several of them play a role in different (painful) pathologies of the somatosensory system, both in animal models and humans (examples are given in Table 1 in Rouwette et al., 2016). This shows that our DIA-MS is able to uncover meaningful candidates: For instance, one of the proteins upregulated in both inflammatory and neuropathic pain, was Dpp4/CD26. It is a cell surface glycoprotein. With its dipeptidyl peptidase activity, it regulates the concentration and activity of a variety of polypeptides, for example, the pain-modulating peptide SP (Guieu et al., 2006). Interestingly, CD26-deficient mice show exaggerated nociceptive responses, whereas in vivo inhibition reduces mechanical hypersensitivity in a model of diabetic neuropathy (Bianchi et al., 2011). Also, heat-shock protein 90b1 (HSP90b1), a molecular chaperone, and another commonly regulated protein in our dataset, has been linked to chronic diabetic neuropathy in mice (Urban et al., 2010, 2012).

Our workflow also detected the regulation of proteins of which mutations are linked to human disorders. For instance, the ATPase ATP1A2, gene mutation underlies familial hemiplegic migraine type II (Fusco et al., 2003), and the ganglioside-induced differentiationassociated protein-1 (Gdap1), mutations causing Charcot-Marie-Tooth 4A neuropathy, an inherited peripheral neuropathy (Baxter et al., 2002).

On the other hand, for roughly $50 \%$ of our candidate proteins we did not find a connection to somatosensation and pain. As evidenced with the examples of proteins already previously linked to painful conditions, our workflow can detect meaningful candidates, thus, these proteins without a previous link to pain could represent interesting candidates to further investigate their role in inflammatory and neuropathic pain, respectively.

We further wanted to validate the differential regulations of proteins detected by MS with complementary methods. Serca1, sarco/endoplasmic reticulum $\mathrm{Ca}^{2+-}$ ATPase 1, was suggested to be upregulated in CFA and downregulated in SNI. Both western blots of membrane-enriched DRG lysates and in situ detection of Serca1 via IHC on DRG and sciatic nerves (from mice/samples exactly treated /prepared as for DIA-MS) confirmed the up- and downregulation, respectively (Rouwette et al., 2016). In addition, we could show that the differences detected in amount of Sercalare mirrored in the alteration of protein activity by measuring intracellular calcium levels in neuronal DRG cultures derived from CFA- and Veh-injected mice (Rouwette et al., 2016). 
A

2,526 proteins detected in all replicates

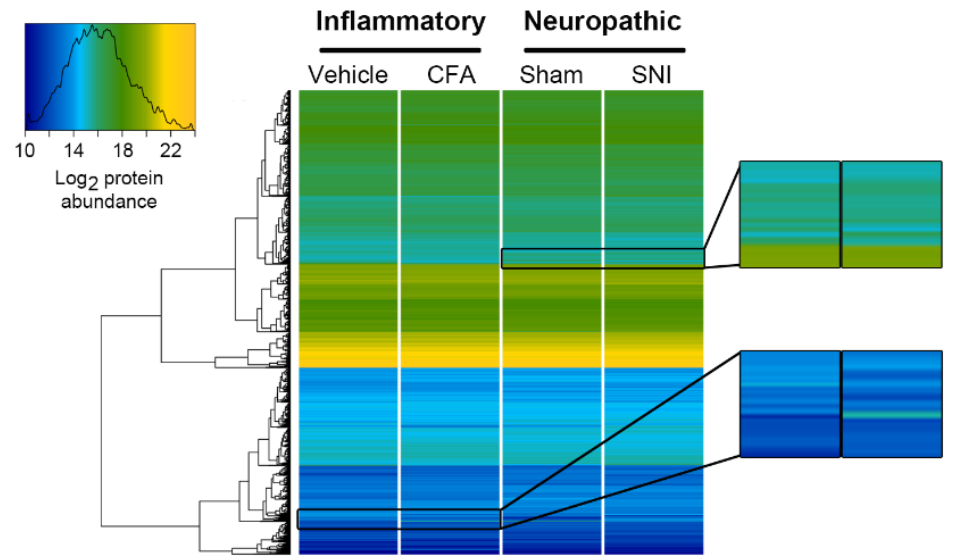

Significantly regulated proteins in: Inflammatory Neuropathic

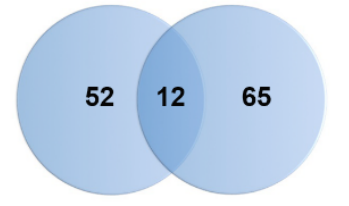

B
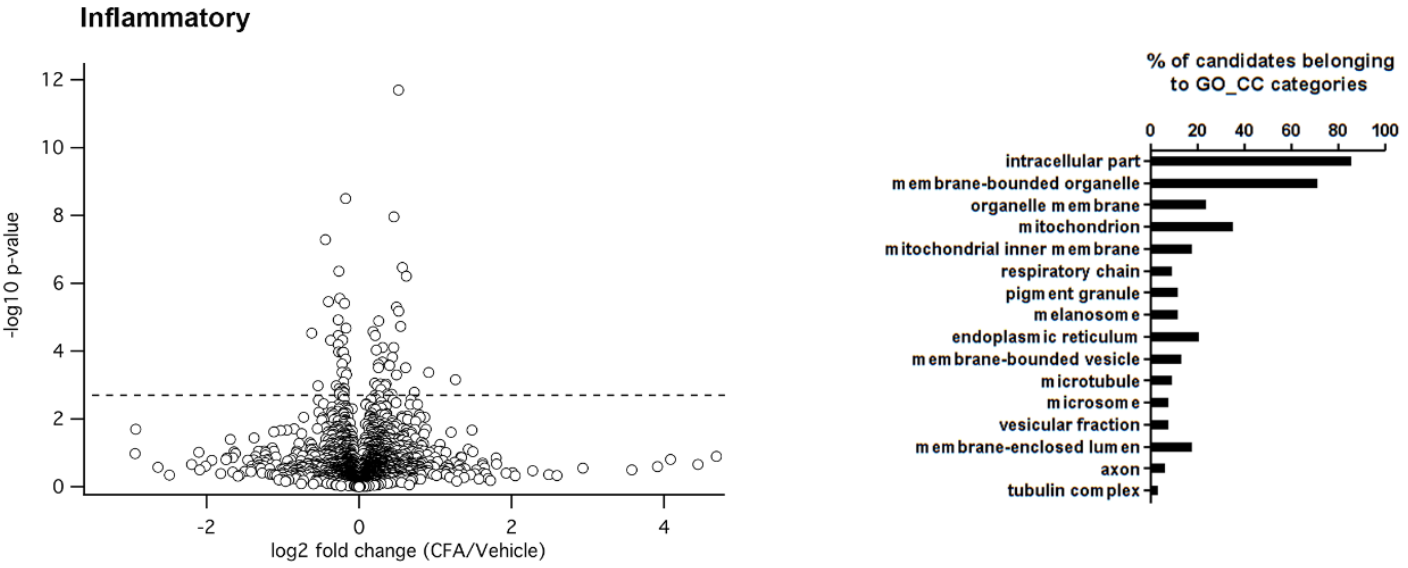

C
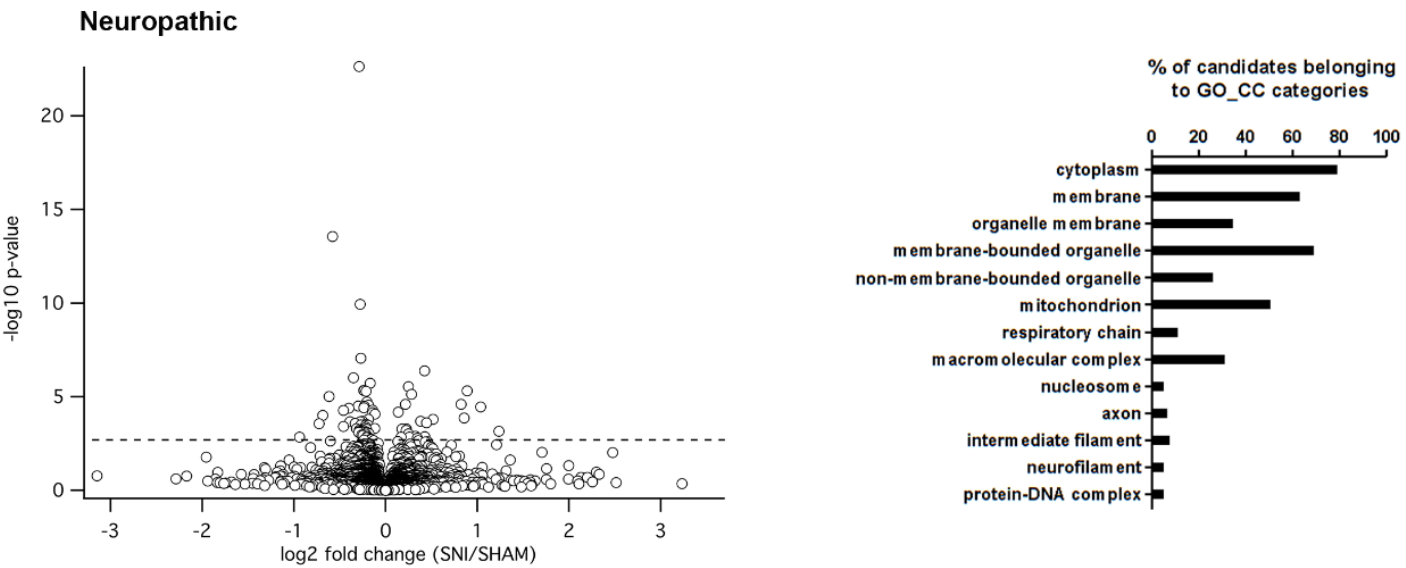

Figure 3.2: Differential regulation of proteins in inflammatory and neuropathic pain

(A) Left, Visualisation of the abundance levels of the same set of 2,526 proteins profiled in all 4 conditions over all replicates in a heatmap (upon unsupervised hierarchical clustering analysis). Abundance changes are represented in the change of the colour. Insets show magnified regions with exemplary proteins that show abundance change. Right, Venn diagram displaying the number of proteins significantly regulated in either inflammatory or neuropathic pain and regulated in both (intersection). (B, C) Left, Volcano plot of all detected and quantified proteins by DIA-MS for inflammatory (CFA vs. Vehicle, B) and neuropathic pain (SNI vs. SHAM, C). The dashed line represents a q-value of 0.05 and thus, proteins above this line are counted as significantly regulated. Right, Bar diagrams representing the fractions of significantly regulated proteins that were annotated with the respective GO cellular component (CC) term. This research was originally published 
in Molecular \& Cellular Proteomics. Rouwette, Sondermann et al.. Standardized profiling of the membrane- enriched proteome of mouse dorsal root ganglia (DRG) provides novel insights into chronic pain. Mol Cell Proteomics. 2015; 15(6), 2152-2168. (C) the American Society for Biochemistry and Molecular Biology.

\subsubsection{Alterations of major cellular signalling networks during chronic pain states}

As only the interplay of several proteins enables cell functioning, we searched for associations among the regulated proteins. Using STRING (Search Tool for the Retrieval of Interacting Genes/Proteins; (Szklarczyk et al., 2016)), we identified links between 56 out of 77 proteins regulated in neuropathic pain, and 40 out of 64 proteins regulated during inflammatory pain. In Fig. 3.3 the proteins were grouped into signalling networks. Several major signalling networks were affected: for instance, structural proteins involved in myelin maintenance and nerve repair processes were downregulated in our SNI model of neuropathic pain. These have been shown to be important for pain hypersensitivity in another nerve injury-mediated model of neuropathic pain (Vacca et al., 2014).

Striking was the significant number of proteins linked to mitochondrial activity in the neuropathic pain paradigm. This is in line with previous studies relating mitochondrial dysfunction to several types of neuropathic pain. Any of the essential and interconnected functions mitochondria fulfil in cells can, if dysregulated, be related to neuropathic pain: their role in (I) the energy metabolism, any disbalance of the electron transport chain (ETC) can have detrimental effects in neurons as they have a high demand of energy (Joseph and Levine, 2006), (II) reactive oxidant species (ROS) production and degradation (oxidative stress)(Kim et al., 2004) and (III) buffering of intracellular calcium (Shishkin et al., 2002). Furthermore, proteins implicated in protein biosynthesis and maturation were affected (Fig. 3.3). Only recently, endoplasmic reticulum (ER) stress has been suggested to be causative factor for pathological pain (Inceoglu et al., 2015). ER stress is triggered by accumulation of unfolded proteins due to disturbances of cellular redox regulation, calcium homeostasis as well as glucose deprivation (Oakes and Papa, 2015). Administration of reagents initiating ER stress causes lasting pain at the injection-site in healthy rodents, which could again be reversed by application of inhibitors of ER stress (Inceoglu et al., 2015). The exact mechanism how the ER stress signalling cascade initiates pain, has yet to be determined.

Also, in the CFA-model of inflammatory pain regulated proteins were associated with protein biosynthesis and maturation as well as mitochondrial activity. This finding had not been reported previously and we decided to investigate the in vivo relevance of these protein networks for CFA-induced nocifensive behaviour in mice. To this end, we searched for reagents previously shown to enable interference with these signalling pathways in vivo, and that neither affect general health nor motor functions in naïve mice.

Many of the regulated mitochondrial proteins are part of the ETC complex I (e.g. Ndufs4 and Ndufv2, Fig. 3.3), thus, we decided for Rotenone, a blocker of the ETC complex I to manipulate mitochondrial functioning. For the pharmacological interference with protein maturation in the ER, we chose PACMA31 (Xu et al., 2012), inhibitor of protein disulfide isomerases (PDI). PDIs mediate protein folding by catalysing the breakage and reformation of disulfide bonds, and three subtypes of them, PDIA1, 3, and 6 showed a profound upregulation in the CFA-evoked inflammatory pain paradigm. 
Twenty-four hours after intraplantar injection of CFA, mice were injected with Rotenone (or vehicle as control) in the ipsilateral hind paw and assessed for their mechanical hypersensitivity. Similarly, another cohort of CFA-injected mice received PACMA31 (or vehicle; via i.p. injection, i.e. systemic administration) and were tested for hypersensitivity. Both compounds attenuated significantly the CFA-induced hypersensitivity to innocuous mechanical stimulation (Fig. 3.4). Whether the observed analgesia is solely dependent on the action of the inhibitors in somatosensory neurons, we cannot determine. Any off-target effects on non-neuronal cells (in the DRG or skin in case of Rotenone, or any tissue reached by the systemic administration of PACMA31) of these compounds would have to be investigated in somatosensory neuron-specific knockout mice. Yet, neither the withdrawal latencies of the non-injected, contralateral hind paw were affected, nor the general motor coordination, which together with observations from other studies using these inhibitors (Joseph and Levine, 2006, 2009; Xu et al., 2012) argues against major off-target effects.

Taken together, we detected considerable and pain-model specific alterations in the abundance of several dozen proteins as well as within functional protein networks with our DIA-MS-based workflow. Importantly, orthogonal validation of the functional relevance of observed alterations in vitro and analysis of mouse pain behaviours demonstrated that significant protein regulations were discovered on the level of both single proteins and signalling networks. 
A

\section{B}
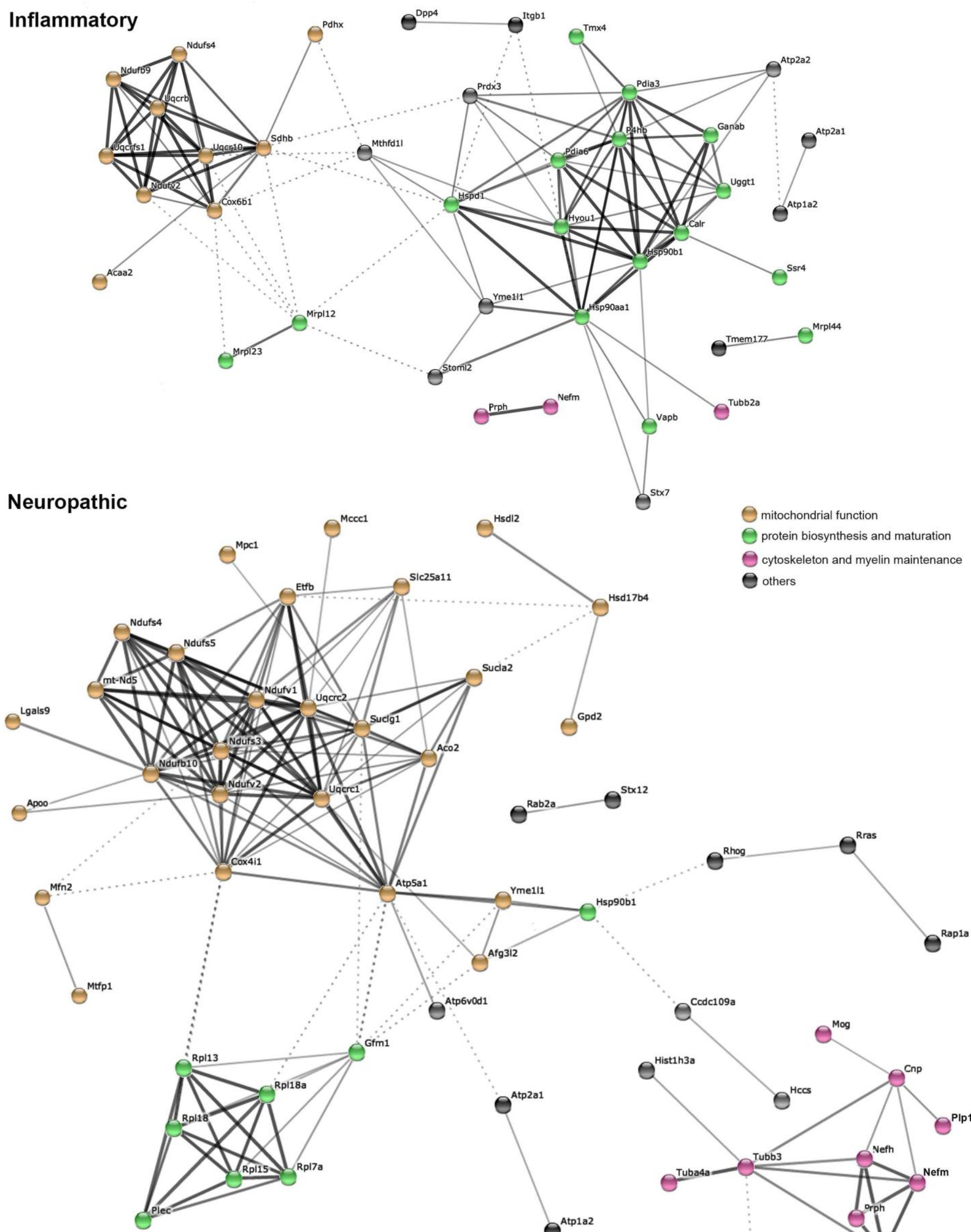

Atp5a1

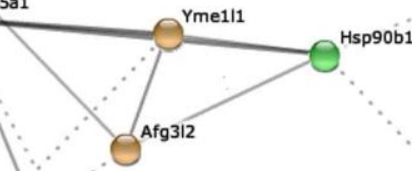

$0^{\text {Atp6rod } 1}$

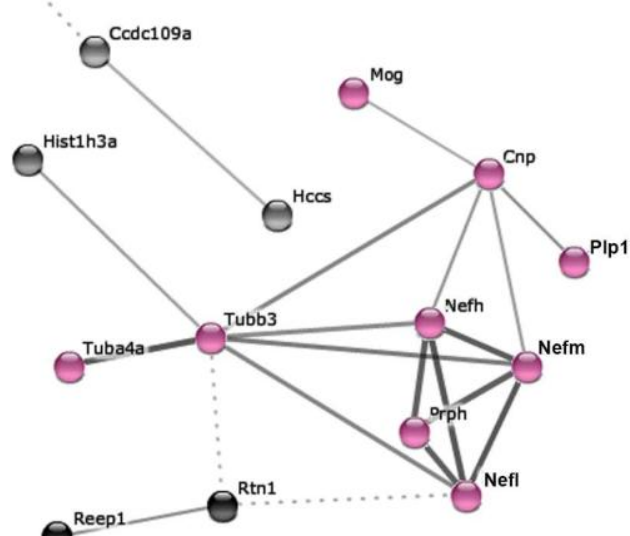


Figure 3.4: Functional association networks of significantly regulated proteins in the different pain paradigms

Interactions were discovered between (A) 40 out of 64 regulated proteins during inflammatory pain and (B) 56 out of 77 regulated proteins during neuropathic pain. Association networks were generated using STRING and thicker lines represent stronger evidence for the association (for details please see section 2.1.7.1). Dashed-lines represent inter-cluster edges. Colours of protein nodes encode protein clusters involved in mitochondrial function, protein biosynthesis and maturation, cytoskeleton and myelin maintenance, and others, respectively. This research was originally published in Molecular \& Cellular Proteomics. Rouwette, Sondermann et al.. Standardized profiling of the membrane- enriched proteome of mouse dorsal root ganglia (DRG) provides novel insights into chronic pain. Mol Cell Proteomics. 2015; 15(6), 2152-2168. (C) the American Society for Biochemistry and Molecular Biology.

A

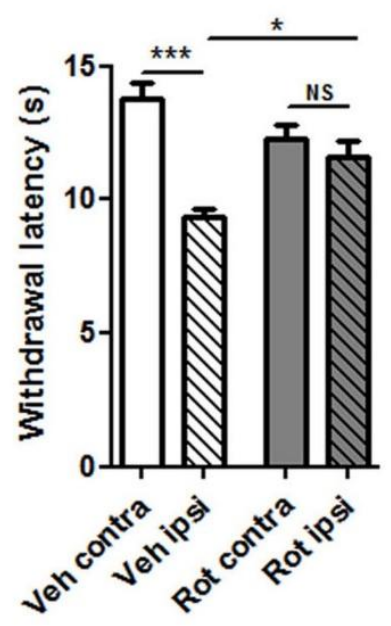

B

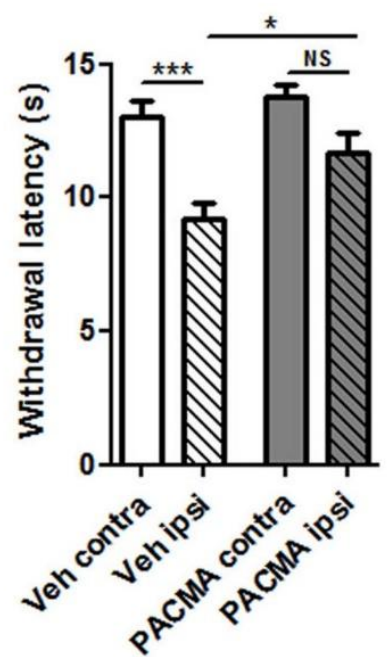

Figure 3.3: Role of mitochondrial and PDI activity in chronic inflammatory pain in mice

(A) Rotenone causes acute analgesia of CFA-evoked chronic inflammatory pain. Withdrawal latencies of the hind paws to mechanical stimulation of CFA-injected animals are depicted. Mice were injected ipsilaterally (ipsi) with either Veh or Rotenone (Rot) $24 \mathrm{~h}$ after CFA-injection, *** $\mathrm{p}<0.001$ and $* \mathrm{p}<0.05, \mathrm{~N}=17 \mathrm{mice} /$ condition, $\mathrm{n}=3$ independent cohorts, one-way ANOVA followed by Bonferroni's multiple comparison tests. (B) PACMA 31-mediated inhibition of PDIs attenuated persistent inflammatory pain. Withdrawal latencies of hind paws from CFA-injected animals are displayed. The mice were injected i.p. with either PACMA 31 (PACMA) or Veh $24 \mathrm{~h}$ after CFA-injection, $* * * \mathrm{p}<0.001$ and $* \mathrm{p}<0.05$, $\mathrm{N}=10$ mice/condition, $\mathrm{n}=2$ independent cohorts, one-way ANOVA followed by Bonferroni's multiple comparison tests. Of note, withdrawal latencies of untreated/contralateral hind paws were similar between PACMA 31 and Veh arguing against unspecific motor effects upon systemic (i.p.) injection of PACMA 31. This research was originally published in Molecular \& Cellular Proteomics. Rouwette, Sondermann et al.. Standardized profiling of the membrane- enriched proteome of mouse dorsal root ganglia (DRG) provides novel insights into chronic pain. Mol Cell Proteomics. 2015; 15(6), 2152-2168. (C) the American Society for Biochemistry and Molecular Biology. 


\subsection{Identification and characterization of TRPV1 protein complexes}

The majority of this section is based on results that have been originally published in PAIN: Sondermann et al. Vtilb promotes TRPVI sensitization during inflammatory pain. 2019; 160(2), 508-527. Copyright (C2019 by the International Association for the Study of Pain. No formal license is required from Wolters Kluwer (publisher) to reprint the figures.

Sensitization of TRPV1 is involved in the development and maintenance of thermal hyperalgesia, a symptom of several pathological pain conditions. Amongst others, proteinprotein interactions modulating the channel's responsiveness to stimuli contribute to this hyperactivity. Targeting interactions that only occur under pathological conditions holds the promise of preventing only the excessive activation of the channel. However, little is known about the regulation and protein scaffold of TRPV1 during different pain states. Several studies reported regulated trafficking of TRPV1 in response to exposure to inflammatory mediators (Camprubí-Robles et al., 2009; Mathivanan et al., 2016; Meng et al., 2016; Morenilla-Palao et al., 2004; Zhang et al., 2005). We searched the genome-wide RNAsequencing data of nociceptors (Thakur et al., 2014) for proteins involved in both stimulated exocytosis and inflammation. One candidate catching our interest was the Vesicle transport through interaction with t-SNAREs homolog 1B (Vti1b). It had already been found to be regulated under inflammatory pain conditions in our proteomics screen of the DRG membrane (section 3.1 and/or (Barry et al., 2018; Rouwette et al., 2016)). Thus, the functional relevance of a potential TRPV1-Vtilb interaction was assessed for TRPV1mediated pain signalling in vitro as well as for pain behaviour in vivo. In recent years, an approach called "functional proteomics" has been used successfully for elucidating protein complexes of ion channels (Schulte et al., 2011). It comprises the affinity-based copurification of the target ion channel with its interacting proteins followed by quantitative tandem-mass spectrometry for the unbiased identification of putative interactors. In the second part of this project, functional proteomics was applied to determine further TRPV1 interactors that are specifically formed under inflammatory pain, a pain state TRPV1 is crucially involved in (Caterina et al., 2000).

\subsubsection{Validation of Vtilb as modulator of TRPV1-mediated nociceptive signalling}

\subsubsection{Co-expression of Vtilb and TRPV1 in sensory neurons}

This section is based on Sondermann et al., 2019.

Vtilb is an endosomal SNARE protein that has been reported to be involved in different steps of both the endocytosis and exocytosis in several different cell types (Antonin et al., 2000; Kreykenbohm et al., 2002; Murray et al., 2005; Offenhäuser et al., 2011).

First, I verified the expression of Vtilb in the sensory neurons in the DRG. Coimmunostainings of lumbar DRG (IDRG) against TRPV1 and Vtilb revealed that Vti1b is expressed in approximately $80 \%$ of all $1 \mathrm{DRG}$ neurons and showed co-expression with 
TRPV1 in 70\% of all TRPV1-positive neurons (Fig. 3.5 A, B). The expression of TRPV1 was not different in DRG from WT mice and Vtilb-KO mice excluding a major effect of Vtilb on TRPV1 expression levels.

Next, I investigated the subcellular localisation of Vtilb in IDRG neurons. Vtilb has been shown to be located to late endosomes and structures of the trans-Golgi network for other cell types (Kreykenbohm et al., 2002). Comparison of the subcellular distribution of Lamp1, a marker for late endosomes (LE) and lysosomes, with Vtilb revealed a partial overlap of the two proteins mainly in the perinuclear region of DRG neurons (Fig. 3.5 C), confirming previously reported localisation of Vtilb to LE and lysosomes in, for example, macrophages (Offenhäuser et al., 2011). Both proteins show a vesicular and/or tubular staining pattern. The expression pattern of the two proteins was comparable in TRPV1-negative and -positive cells (Fig. 3.5 C). Of note, the TRPV1 immunostaining was dispersed throughout the whole cytosol which made it impossible to determine any subcellular co-localisation of both proteins of interest.

Figure 3.5: Co-expression of TRPV1 and Vti1b in IDRG neurons

(A) Representative images of co-immunostainings against TRPV1 and Vtilb on IDRG sections from wild type mice. Coexpressing neurons are marked with white asterisks in the merged image of the wild type (inset, TRPV1-expressing neurons in magenta and Vti1b in cyan). Inset represents a magnification of the white box. Scale bar, $50 \mu \mathrm{m}$. (B) Quantitation of the TRPV1 and Vtilb expression in DRG neurons from either Vtilb-KO (KO) or wild type (WT) mice. There is no significant difference in the TRPV1-positive neuronal population between conditions (TRPV1, WT: $27.5 \pm 2.0 \%$ vs. KO: $28.5 \pm 2.4 \%$, ns) suggesting that the knockout of Vtilb (Vtilb, WT: $77.7 \pm 3.3 \%$ vs. KO: $0.65 \pm 0.4 \%, * * * p<0.0001$ ) does not have a major effect on TRPV1-expression. Of the TRPV1-expressing neurons, $70.8 \pm 5.6 \%$ were also expressing Vtilb (Vti1b/TRPV1) in the WT as compared to the KO $0.6 \pm 0.6 \%$ (*** p < 0.0001). Count of neurons is given in the columns, $\mathrm{n}=2-3$ mice, Kruskal-Wallis test followed by Dunn's multiple comparison test. Data represented as mean \pm SEM. (C) Coimmunostainings of dissociated mouse IDRG neurons against TRPV1, Vti1b and Lamp1. White asterisk indicates TRPV1positive cell. Nuclei are visualized by DAPI staining (blue). Both TRPV1-positive and TRPV1-negative neurons express Vtilb (cyan in overlay) and Lamp1, a marker for late endosomes (magenta in overlay). Their staining pattern shows a partial overlap of the labelling for both proteins. Scale bar, $15 \mu \mathrm{m}$. From Sondermann et al., 2019. 
A

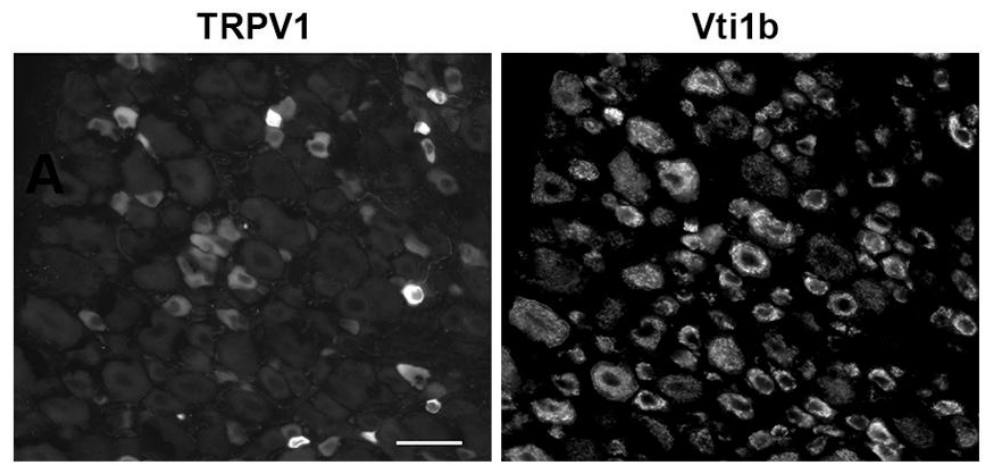

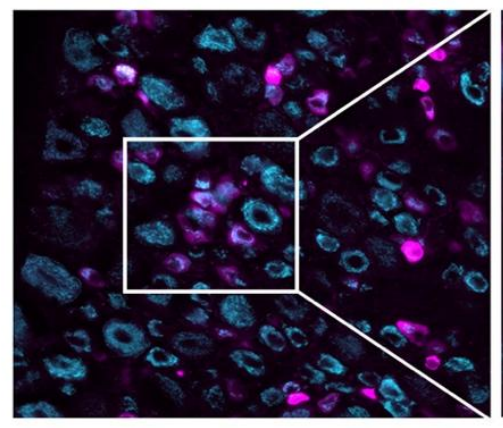

TRPV1 \& Vti1b

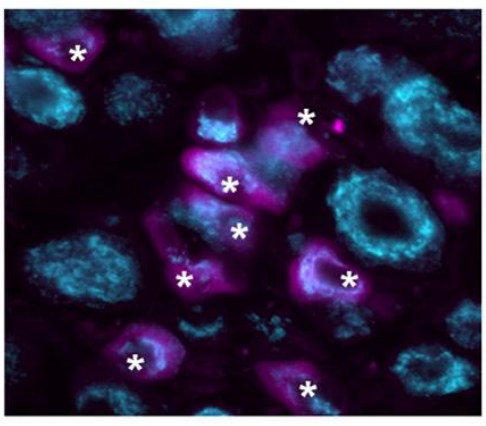

Inset

B

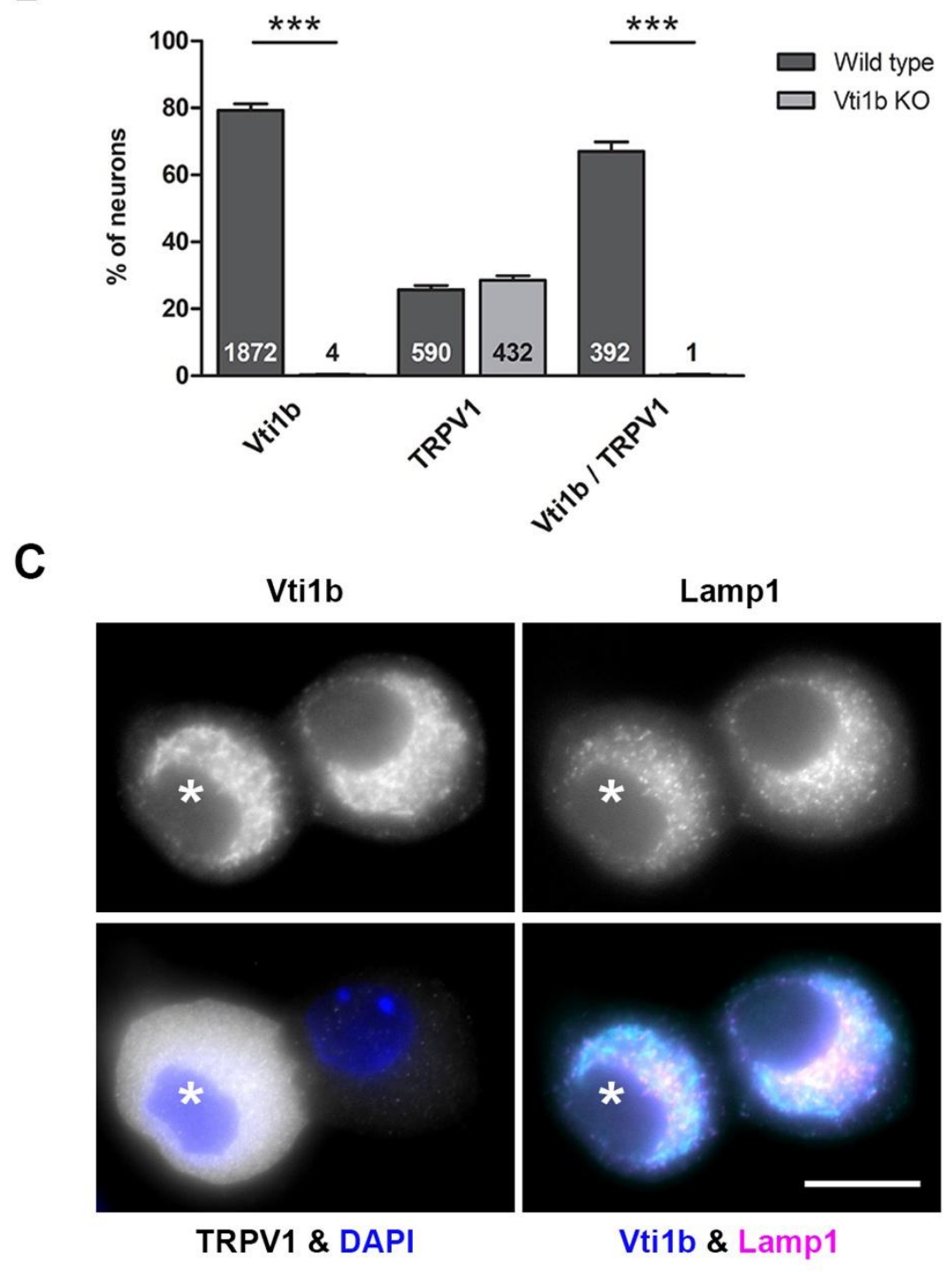




\subsubsection{Vtilb modulates TRPV1 tachyphylaxis in heterologous expression systems}

This section is based on Sondermann et al., 2019.

The live labelling and calcium imaging experiments in HEK cells were performed by Master Student Allison Barry under the author's teaching and supervision and are summarized in her Master thesis. The electrophysiological recordings were done by Niklas Michel, a Ph.D. student of the Schmidt lab.

To investigate the nature of the interaction of TRPV1 and Vtilb, we made use of heterologous expression systems. HEK293 cells are easier to manipulate and obtained results might show a better reproducibility due to the homogeneity of the cells, as compared to the difficult-to-transfect and heterogeneous population of cultured DRG neurons of which only a fraction is expressing TRPV1, and in varying amounts.

As stated above, Vti1b is involved in trafficking processes and could potentially also regulate trafficking of the TRPV1 channel to and from the plasma membrane. We therefore asked whether Vtilb expression level influences the surface expression of TRPV1. To this end, we performed live labelling of HEK cells, overexpressing TRPV1-YFP and Vti1b, or TRPV1YFP alone, using an antibody against an extracellular domain of the TRPV1 channel. As the antibodies cannot penetrate the intact cell membrane (within the limited time of incubation during the staining protocol), only TRPV1 channels residing at the plasma membrane are labelled. In YFP-positive HEK cells, we observed a clear punctuated staining at the membrane marking the plasma membrane TRPV1 population. However, upon co-expression of Vti1b the membrane TRPV1 staining was noticeably reduced (Fig. 3.6 B), even though the overall number of TRPV1 expressing cells (i.e. YFP-positive cells) did not differ significantly (85 and 90 cells from 3 independent cultures were analysed, 21 regions per condition). This suggests that Vtilb regulates the trafficking to the surface, possibly increasing endocytosis or inhibiting exocytosis of the channel.

Apart from regulating the availability of functional TRPV1 at the membrane, Vti1b could also modulate gating properties of the channel TRPV1. Therefore, we performed electrophysiological recordings of HEK cells transiently transfected with either TRPV1 + mock or TRPV1 + Vti1b. Cells were subjected to $500 \mathrm{~ms}$ voltage ramps, ranging from -100 $\mathrm{V}$ to $+100 \mathrm{~V}$, in whole-cell patch clamp mode and applied a single pulse of $100 \mathrm{nM}$ capsaicin (Fig. 3.6 A). As expected, we found the TRPV1-mediated current to be outwardly rectifying and saw an increase upon capsaicin stimulation (Caterina et al., 1997). When comparing the 2 conditions, we observed a significant decrease in the current density amplitude for the TRPV1 and Vtilb co-expressing cells (both before and after capsaicin stimulation). Neither the slope of the I-V curve nor the reversal potential differed between conditions, suggesting no change in the ion selectivity or voltage dependency of the channel. An explanation for the decrease in current density could be less TRPV1 at the membrane which is supported by the findings of the live labelling.

Next, we set out to investigate the functional relevance of the interaction by means of ratiometric calcium imaging. TRPV1 is permeable to calcium, therefore, rises in intracellular calcium levels correlate with channel activity. Another prominent feature of TRPV1 is its rapid desensitization to agonist stimulation, in particular to repetitive pulses (also referred to as tachyphylaxis (Koplas P. A. et al., 1997)). To observe potential changes in the 
desensitization behaviour, we challenged HEK cells with 6 pulses of $100 \mathrm{nM}$ capsaicin (Caps) followed by a saturating pulse of capsaicin $(1 \mu \mathrm{M}$; see 2.1.5.8 for details). The HEK cells were overexpressing either TRPV1 + Vtilb or only TRPV1 alone. Stimulation protocols with several consecutive stimuli are often used in literature to study TRPV1 desensitization phenomena in vitro and this 6-pulse protocol was modified from Bonnington and McNaughton as well as Hanack et al. (Bonnington and McNaughton, 2003; Hanack et al., 2015).

In Fig. 3.6 C, the averaged traces of responder to the capsaicin stimulation are depicted, showing noticeable differences in the changes of intracellular level of calcium between the two experimental groups upon application of capsaicin (for amplitude data to each pulse see Appendix Fig. 7.1 A). The percentage of responders to each of the capsaicin pulses, especially notable for the initial $1^{\text {st }}$ capsaicin pulse (\% is referred to the count of all TRPV1expressing cells, i.e. sum of cells responding to one or more capsaicin pulses) was lower in the TRPV1 + Vti1b group. This suggest that co-expression of Vtilb decreases the amount of functional TRPV1 at the membrane, which is in line with the results of the live labelling and whole cell recordings above. However, this observation could simply stem from artefacts due to overexpression of the protein which may compromise the cells' health and ability to express and target the proteins to their correct location.

To investigate differences in the tachyphylaxis behaviour, we compared the number of cells responding to the $1^{\text {st }}$ pulse to those responding to the $6^{\text {th }}$ pulse. We found that the fraction of non-responder (i.e. desensitized cells) is not different in cells co-expressing TRPV1 and Vti1b, while cells expressing only TRPV1 exhibited the expected tachyphylaxis, i.e. less cells responding to the $6^{\text {th }}$ than to the $1^{\text {st }}$ pulse (Fig. 3.6 D left). The changes in number of responders were supported by similar changes in the amplitudes (Fig. 3.6 C, D right).

As mentioned above the overexpression of proteins could impair cell health, thus, we applied ATP - activating endogenously expressed purinergic channels in the HEK cells (He et al., 2003) - at the end of the protocol to compare the overall health status of the two conditions. Yet, no difference was observed (Appendix Fig. 7.1), excluding that a general compromised cellular health caused the observed differences.

To sum up, on one hand, Vtilb co-expression resulted in an attenuated responsiveness to capsaicin that could be attributed to less functional TRPV1 at the membrane already prior to the $1^{\text {st }}$ application of the agonist. One the other hand, the typically observed desensitization/tachyphylaxis of TRPV1, measured by a decrease in cells responding to consecutive pulses as well as a decrease in the response amplitude, is diminished and/or absent in TRPV1 and Vtilb co-expressing cells. Both could be explained with impaired endo- or exocytosis of the channel to the membrane. 
A

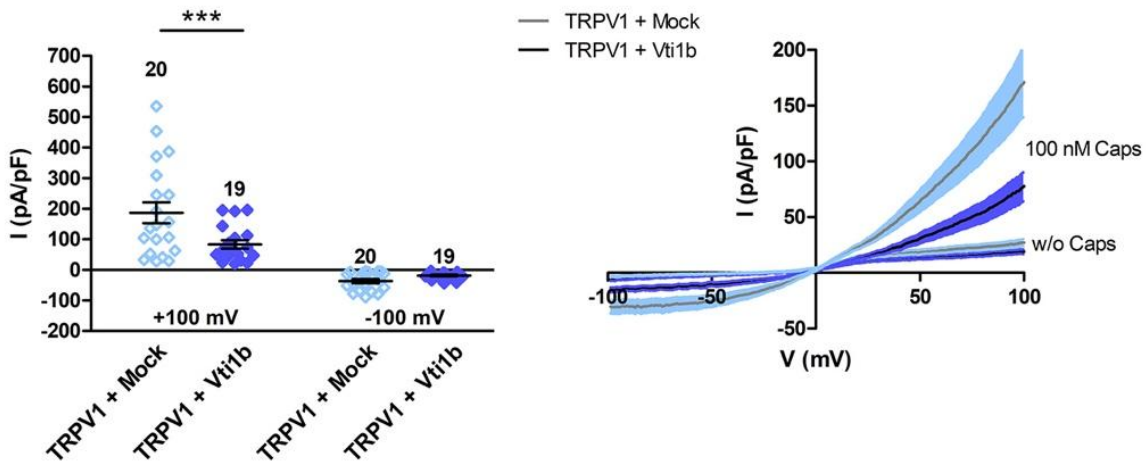

B
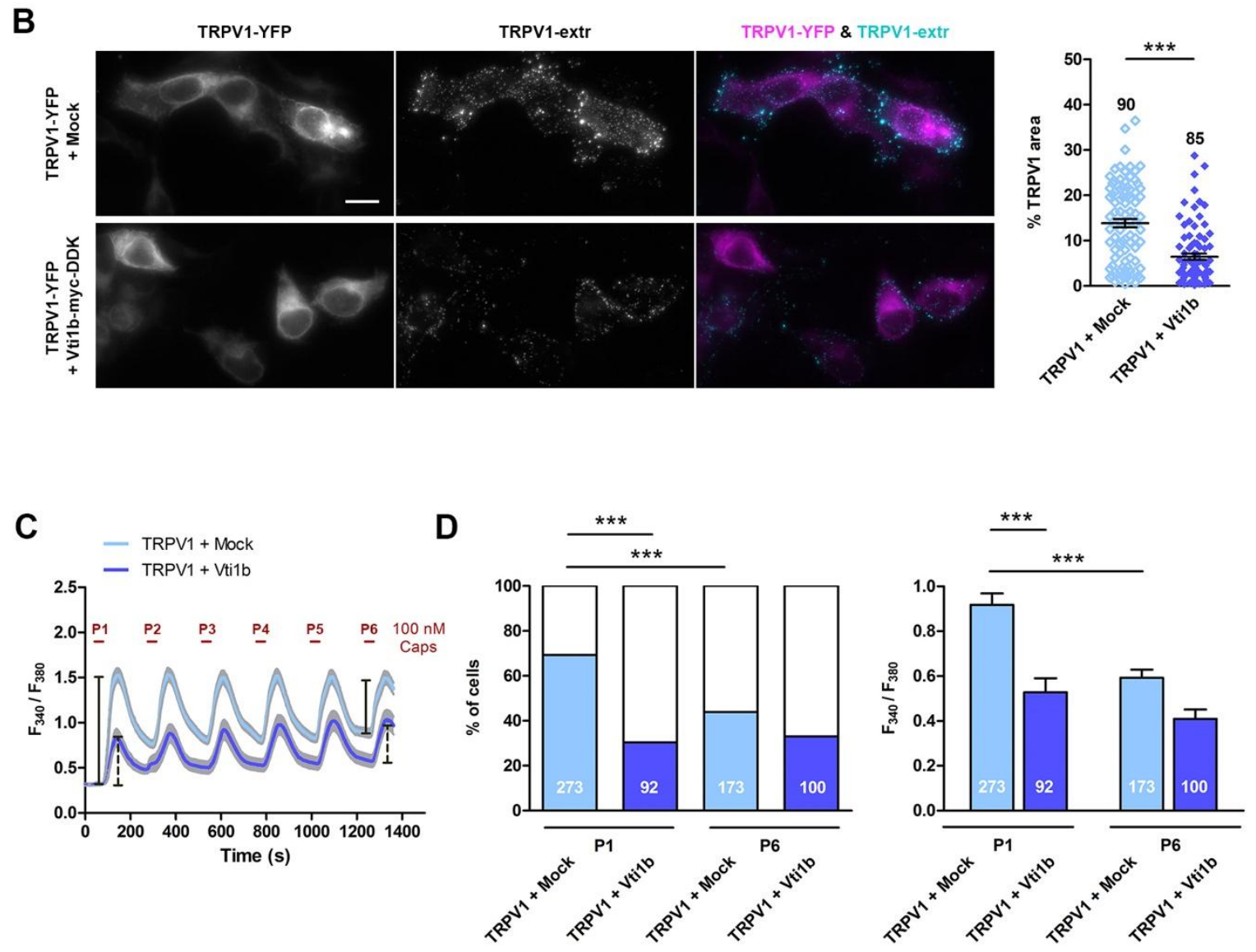
Figure 3.6: Overexpression of Vti1b alters surface expression, function and desensitization of heterologously expressed TRPV1 channels

\begin{abstract}
(A, left) Peak current densities evoked by application of $100 \mathrm{nM}$ capsaicin (Caps). HEK293 cells were transiently transfected with either TRPV1-YFP + Mock or TRPV1-YFP + Vti1b. For $+100 \mathrm{mV}$, *** p $=0.0003$, and for $-100 \mathrm{mV}$, ns, $\mathrm{p}=0.2755$, two-way ANOVA followed by Holm-Sidak's multiple comparisons test. (A, right) Averaged I-V relationships before (w/o Caps) and after the application of $100 \mathrm{nM}$ Caps in HEK293 cells overexpressing either TRPV1-YFP + Mock and TRPV1-YFP + Vti1b, $\mathrm{n}=11-13$ independent cultures. (B, left) Representative images of live-labelling of TRPV1 at the plasma membrane. HEK293 cells were transiently transfected with either TRPV1-YFP + Mock or TRPV1-YFP + Vti1b and were live-labelled with an antibody against extracellular epitope of TRPV1 for $10 \mathrm{~min}$ at $37^{\circ} \mathrm{C}$. Only the surface population of TRPV1 was labelled by the TRPV1 antibody (TRPV1-extr). Upon co-expression of Vti1b less YFP-positive cells show intense surface labelling. Scale bar, $15 \mu \mathrm{m}$. (B, right) Sample quantification of (B, left) represented as fraction of membrane TRPV1-positive area (to total cell area) in YFP-positive cells. *** $\mathrm{p}<0.0001, \mathrm{n}=3$ independent cultures; Mann-Whitney test. (C) Representative traces of rises in intracellular calcium as fluorescence absorbance ratio 340/380 i.a.u.. Graph displays averaged traces (mean \pm SEM) of HEK293 cells transiently transfected with either TRPV1 + Mock (light blue, $\mathrm{N}=91$ cells) or TRPV1 + Vtilb (dark blue, $\mathrm{N}=27$ cells) that were able to respond to all 6 consecutive Caps pulses. When comparing the amplitudes of P6 to P1 (indicated by black vertical bars) within one condition, it becomes apparent that for the TRPV1 + Vtilb condition nearly no tachyphylaxis is seen. (D, left) The fraction of responders (colored) and non-responders (white) of TRPV1-expressing cells are shown in stacked bar graphs for the P1 and P6 pulse for each condition. For P1 TRPV1 + Mock vs. P1 TRPV1 + Vti1b: *** p < 0.0001; for P1 TRPV1 + Mock vs. P6 TRPV1 + Mock: *** $\mathrm{p}<0.0001$; for P1 TRPV1 + Vti1b vs. P6 TRPV1 + Vti1: ns, $\mathrm{p}=0.5411, \mathrm{n}=3$ independent cultures, Fisher's exact test. (D, right) Averaged response amplitudes of TRPV1-expressing cells are displayed for the P1 and P6 pulse for each condition. For P1 TRPV1 + Mock vs. P1 TRPV1 + Vti1b: *** p < 0.001; for P1 TRPV1 + Mock vs. P6 TRPV1 + Mock: *** $\mathrm{p}<0.0001$; for P1 TRPV1 + Vti1b vs. P6 TRPV1 + Vti1b: $n s, \mathrm{p}=0.2209, \mathrm{n}=3$ independent cultures; one-way ANOVA + Holm-Sidak multiple comparisons test. A-C \& D, left: data are displayed as mean \pm SEM and cell count above scatter/ in column. From Sondermann et al., 2019.
\end{abstract}

\title{
3.2.1.3 Modulation of Vtilb expression levels alters TRPVI-dependent sensitization of sensory neurons in vitro
}

This section is based on Sondermann et al., 2019.

To explore a functional relevance for the proposed TRPV1-Vti1b interaction on TRPV1mediated nociceptive signalling, we moved from the heterologous expression system to dissociated cultures of sensory neurons from mouse IDRG. We modulated the Vtilb expression level by siRNA-mediated knockdown in cultures derived from mice either injected with CFA, or Veh as controls, to mimic inflammatory pain. Ratiometric calcium imaging with a similar $6 \times 100 \mathrm{nM}$ capsaicin pulse protocol as in section 3.2.1.2 was performed to assess differences in the capsaicin-evoked desensitization behaviour of TRPV1-expressing sensory neurons (Fig. 3.7). The neurons were imaged $72 \mathrm{~h}$ after transfection with siRNA against Vti1b, or negative control (Ctlr) siRNA (see Fig. 3.7 A for proof of successful knockdown).

The siRNA-mediated knockdown of Vtilb did not lead to statistically significant differences in the percentage of TRPV1-expressing neurons responding to the initial capsaicin pulse (P1, Fig. 3.7 C), neither in the Veh group nor in the CFA group. Also, the response amplitudes to the first pulse were not different between groups or conditions (Fig. 3.7 D).

To take a closer look at the potential influence of the reduced Vtilb level on the sensitization of TRPV1 expressing neurons, I wanted to assess whether tachyphylaxis, i.e. the fraction of cells responding to the $6^{\text {th }}$ pulse, differed between groups and conditions (Fig. 3.7 E, F). There was a noticeable effect of CFA on the desensitization of the neurons already for the control condition. Only $22.5 \%$ of all TRPV1-expressing neurons responded to the $6^{\text {th }}$ pulse in the Veh group (Veh c) vs. 51.1\% in the CFA group (CFA c, Fig. 3.7 E), suggesting that 
CFA-mediated sensitization of TRPV1 channels can override the tachyphylaxis observed in DRG neurons derived from Veh-injected mice. In CFA-treated cultures the knockdown of Vtilb (CFA si) resulted in tachyphylaxis, i.e. significantly less responder to the $6^{\text {th }}$ pulse as compared to CFA c. In addition, the amplitude of the responders was reduced in CFA si as compared to CFA c. Overall, the magnitude of tachyphylaxis in CFA si was similar to that seen in the Veh si group (Fig. 3.7 E). Of note, the overall number of TRPV1-expressing neurons, i.e. number of neurons responding to one or more capsaicin pulses, was not changed between groups or conditions. This suggests - together with the finding that responses to the initial capsaicin pulse (P1) were not different - that the modulation of Vti1b expression does not compromise the general TRPV1-expression in sensory neurons.

Taken together, these data indicate that Vtilb promotes the sensitization of TRPV1expressing IDRG neurons treated with CFA. Since Vtilb has been suggested to play a role at different steps of intracellular trafficking, one working hypothesis was that the decrease in expression of Vtilb leads to an increase in the stimulated endocytosis of TRPV1 after repetitive stimulation under inflammatory conditions. Therefore, I decided to inhibit clathrin-dependent endocytosis to see whether this could rescue the decrease of CFAmediated sensitization when Vtilb is downregulated. To this end, I applied dynasore (inhibitor of dynamins, a group of GTPases responsible for the scission of clathrin-coated pits from the plasma membrane (Macia et al., 2006)) to neuronal IDRG cultures from CFAinjected mice, in which I downregulated Vtilb expression via siRNA, followed by calcium imaging. The same stimulation protocol as above was used, except that I applied dynasore, which acts within seconds after application, in the washout between the $5^{\text {th }}$ and $6^{\text {th }}$ capsaicin pulse until the end of the $6^{\text {th }}$ pulse. In Fig. 3.7 E the effect of dynasore on the fraction of cells responding to the $6^{\text {th }}$ pulse is displayed, showing that with addition of dynasore the sensitization (i.e. an increase in responders to the $6^{\text {th }}$ pulse) is restored. The restored sensitization was also reflected in the response amplitudes (Fig. 3.7 F). Preventing internalization of TRPV1 via clathrin-mediated endocytosis successfully rescued the sensitization of the Vti1b knockdown, suggesting Vti1b has a role in regulating the activitydependent trafficking of TRPV1. However, we cannot tell at which step of the trafficking Vtilb comes into play. Previously, Vtilb has been related to both stimulated exocytosis as well as lysosomal degradation via mediating the fusion of LE and lysosomes in immune cells (Dressel et al., 2010; Murray et al., 2005; Offenhäuser et al., 2011). Further calcium imaging experiments using for example, lysosomal inhibitors would be needed to address this point. 
A
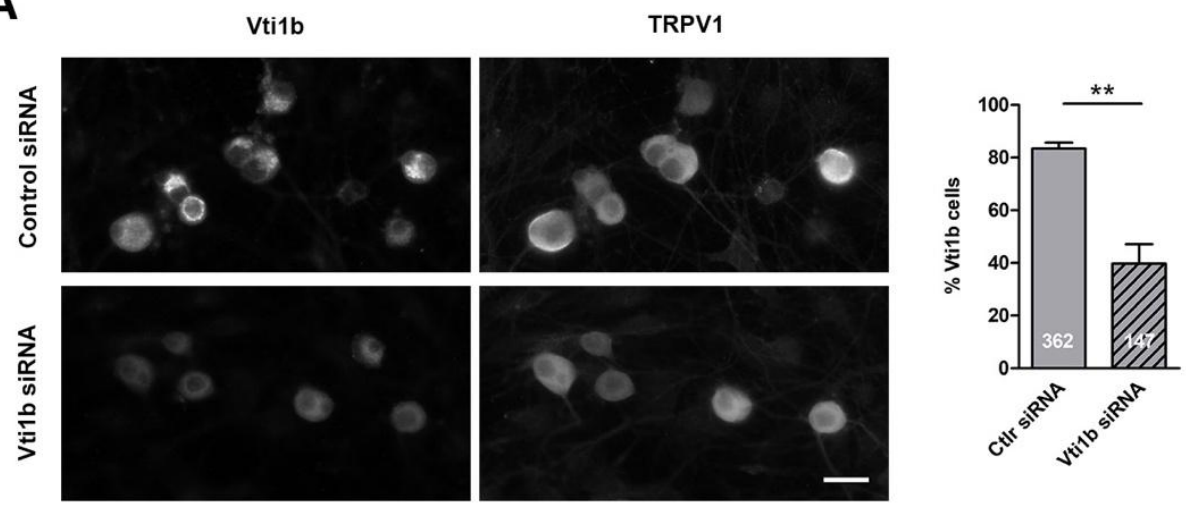

B

Veh - control siRNA

Veh - Vti1b siRNA
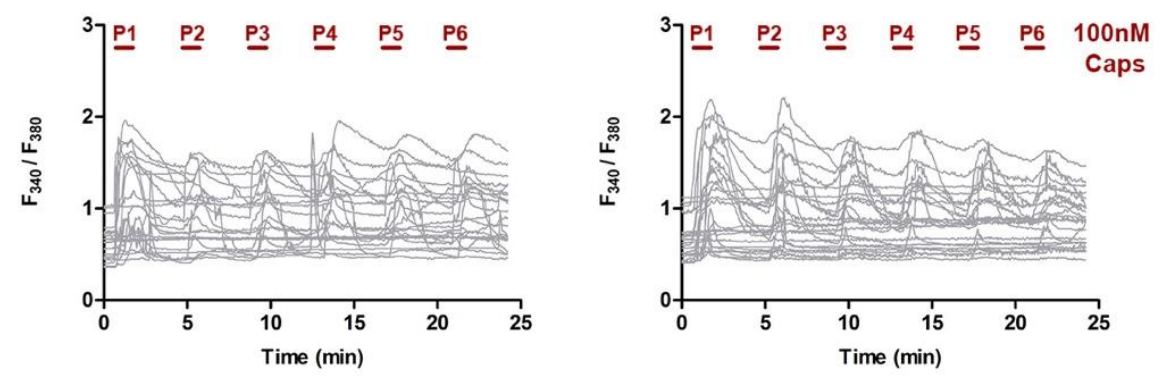

CFA - control siRNA

CFA - Vti1b siRNA
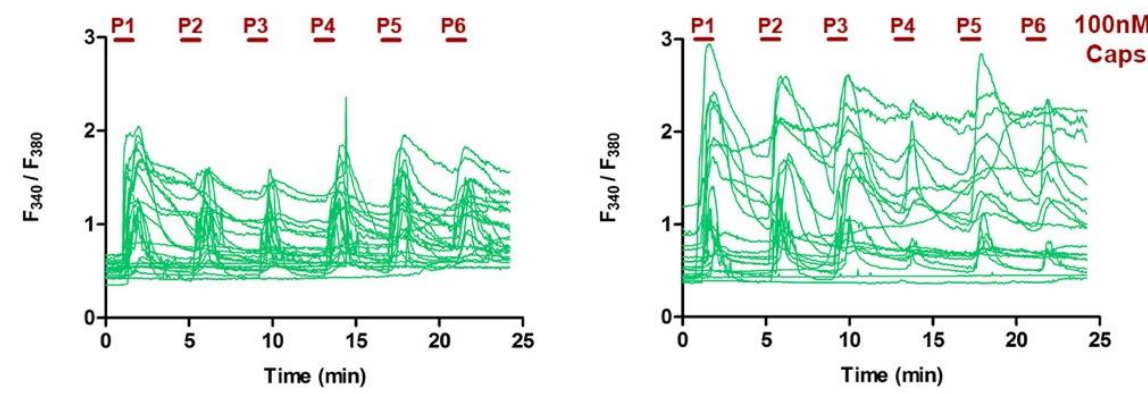

C

D
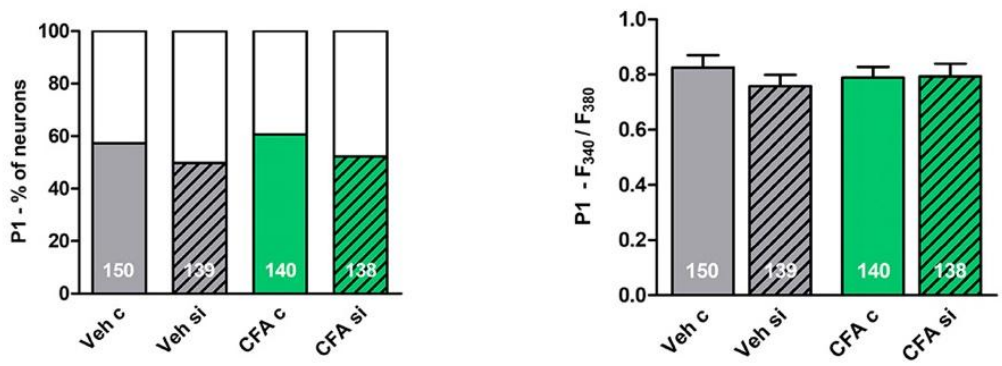

E

F
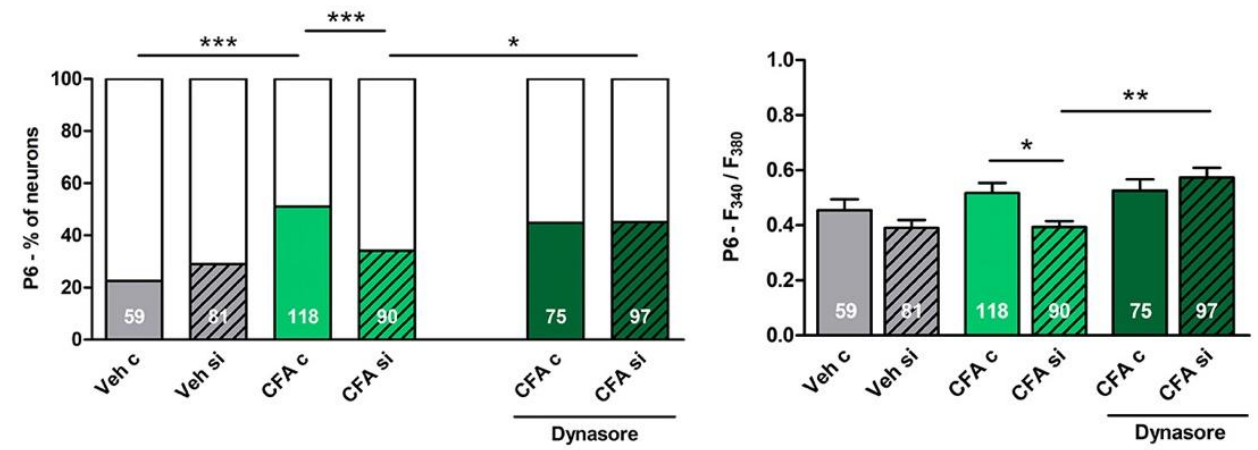


\section{Figure 3.7: Vtilb knockdown affects CFA-induced sensitization of TRPV1-expressing sensory neurons}

(A, left) Representative images of primary IDRG cultures stained against Vtilb and TRPV1 72h after transfection with either Vtilb siRNA (lower panels) or negative control siRNA (upper panels). Scale bar, $25 \mu \mathrm{m}$. (A, right) Quantification of efficient siRNA-mediated downregulation of Vtilb expression. The percentage of Vtilb-positive neurons is significantly reduced upon siRNA-mediated knockdown, $* \mathrm{p}<0.05, \mathrm{n}=3$ independent culture preparations, unpaired Student's t-test. (B) Representative traces of rises in intracellular calcium (fluorescence ratio 340/380 i.a.u.) in dissociated cultures of IDRG from Veh-/CFA-injected mice, treated with Vtilb siRNA (si) and control siRNA (c), respectively. They were challenged with 6 pulses of $100 \mathrm{nM}$ capsaicin (P1-P6, Caps). Note that cells are only counted as "responder" if the rise in calium exceeds 20\% of the baseline for each pulse. (C) Fraction of responders (to TRPV1-expressing neurons, colored) and nonresponders (white) to the $1^{\text {st }}$ Caps pulse $(\mathrm{P} 1), \mathrm{ns}, \mathrm{n}=4$ independent cultures; Fisher's exact test. (D) Averaged response amplitudes (peak of fluorescence ratio 340/380 above baseline) to P1; $n=4$ independent cultures; one-way ANOVA followed by Holm-Sidak's multiple comparisons test. (E) Fraction of responders (colored) and non-responders (white) to the $6^{\text {th }}$ capsaicin pulse (P6). Veh c vs. CFA c: $* * * p<0.0001$; Veh si vs. CFA si: ns; CFA c vs. CFA si: $* * * p=0.0002$. Incubation with Dynasore between P5 and P6: CFA si vs. Dynasore CFA si: $* p=0.0146, n=3-4$ independent cultures, Fisher's exact test. (F) Averaged response amplitudes (peak of fluorescence ratio 340/380 above baseline) to P6. CFA c vs. CFA si: ${ }^{*} \mathrm{p}=0.0349$; CFA si vs. Dynasore CFA si: $* * \mathrm{p}=0.001$, cell counts in the columns, $\mathrm{n}=3-4$ independent cultures, one-way ANOVA followed by Holm-Sidak's multiple comparisons test. A, D, F: data displayed as mean \pm SEM; A, C-F: count of neurons in columns; if not indicated otherwise, all comparisons ns. From Sondermann et al., 2019. 


\subsubsection{Vtilb does not interact with TRPAl in sensory neurons}

TRPA1 experiments were performed by Master Student Allison Barry under the author's teaching and supervision and are summarized in her Master thesis.

Nearly all TRPA1-expressing DRG neurons co-express TRPV1 (Story et al., 2003) and several lines of evidence suggest an interaction of both channels (Akopian et al., 2007; Salas et al., 2009; Staruschenko et al., 2010; Weng et al., 2015). We therefore investigated whether Vtilb also modulates TRPA1-mediated activity in DRG neurons. RNAi-mediated knockdown of Vtilb in mouse IDRG cultures was followed by ratiometric calcium imaging (Fig. 3.8). We stimulated the cells with a $25 \mu \mathrm{M}$ mustard oil (MO) pulse, followed by a $50 \mu \mathrm{M}$ MO pulse and subsequently, the cells were depolarized with $\mathrm{KCl}$ to determine the total population of neuronal cells. Neither the percentage of responders to each pulse, nor the respective response amplitudes, nor the total number of cells responding to one or more MO pulses (i.e. TRPA1-expressing neurons) was changed between conditions (Fig. 3.8 AC). Also, the desensitization of TRPA1 did not show any significant changes as presented in Fig. 3.8 D by comparing the fraction of neurons responding to both MO pulses. This suggests that Vtilb is not interacting with TRPA1. This was further supported by results from a mass spectrometric screening for TRPA1 interactors performed in my host laboratory, in which Vtilb was not detected (Luca Avenali, personal communication). A B
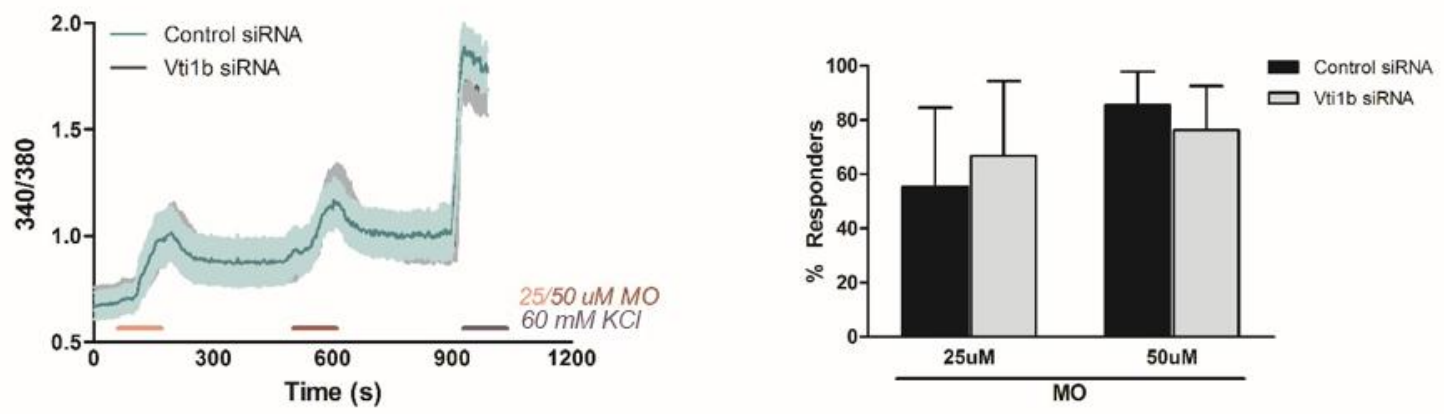

C

D
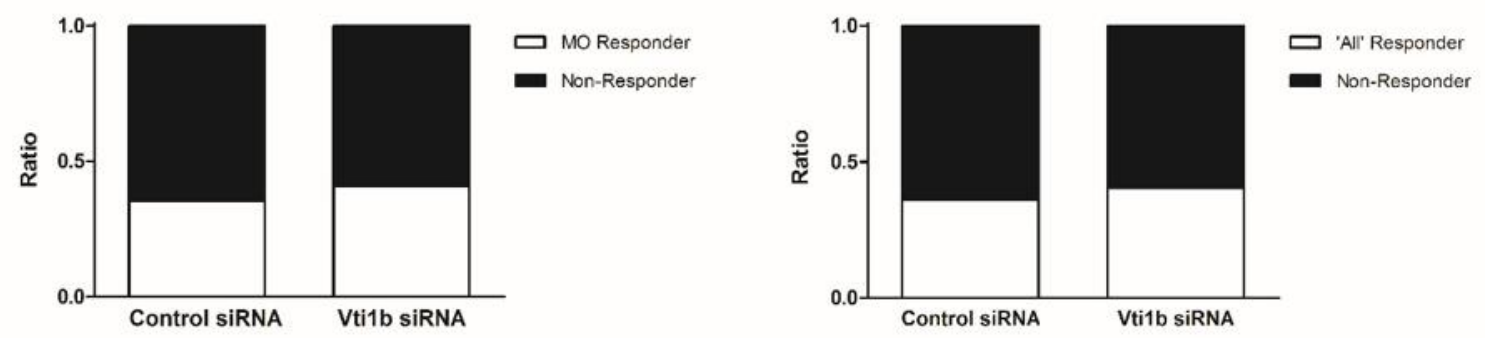

Figure 3.8: Knockdown of Vti1b does not influence TRPA1-mediated neuronal activity

(A) Representative traces of rises in intracellular calcium (fluorescence absorbance ratio 340/380 i.a.u.). Graph displays averaged traces (mean \pm SEM) of DRG neurons transiently transfected with either control siRNA (green) or Vtilb siRNA (black), $72 \mathrm{~h}$ after transfection, that responded to both the $25 \mu \mathrm{M}$ and $50 \mu \mathrm{M}$ MO application; $\mathrm{N}=27$ neurons for control and $\mathrm{N}=38$ for Vtilb siRNA. (B) Percentage of responder (to TRPA1-expressing cells) to each MO pulse (mean \pm SEM). (C) Proportion of cells responding to any of the MO pulses (i.e. TRPA1-expressing cells); $\mathrm{N}=204$ for control and $\mathrm{N}=$ 219 for Vtilb siRNA. (D) Fraction of cells responding to both MO pulses; N $=72$ for control siRNA and $\mathrm{N}=89$ for Vtilb siRNA. None of the data showed significant differences between control and Vtilb siRNA; two-way ANOVA (B, C) and Fisher's exact test $(\mathbf{C}, \mathbf{D})$. Data from $\mathrm{n}=2$ independent cultures. Graphs adapted with permission from A. Barry. 
Results $\mid 66$

\subsubsection{In vivo knockdown of Vtilb attenuates thermal hyperalgesia in an inflammatory pain model}

The major part of this section is based on Sondermann et al., 2019.

As the results suggest a functional relevance for the Vti1b-TRPV1 interaction, I tested whether Vti1b also modulates TRPV1- dependent pain behaviour in mice.

First, Vtilb-deficient mice (a global, constitutive KO; (Atlashkin et al., 2003)) were assessed for basal pain thresholds (both mechanical and thermal), acute capsaicin-evoked pain behaviour and the development of thermal hyperalgesia in the CFA model of inflammatory pain. However, no significant differences in any of the behavioural tests was observed (Appendix Fig. 7.3). The missing of a phenotype in constitutive KO mice can often be related to compensation by a homologous protein. In the case of Vtilb, the closely-related Vtila (30\% amino acid homology) could be a candidate for a compensation, as the double $\mathrm{KO}$ of Vti1b and Vti1b is perinatal lethal (Kunwar et al., 2011), whilst deletion of one of them leads to a minor phenotype (Atlashkin et al., 2003).

To circumvent this problem, I established the virus-mediated local knockdown of Vtilb in IDRG. Adeno-associated virus, serotype 6 (AAV6) encoding GFP-coupled miRNA targeting Vtilb (and as control scramble miRNA-GFP) was injected unilaterally into the sciatic nerve of mice. After 3 weeks the successful knockdown of Vti1b was confirmed by immunohistochemistry against Vti1b and GFP on IDRG (Fig. 3.9 A). On average, 15-18\% of all IDRG neurons showed GFP expression (GFP, Fig. 3.9 B), i.e. were successfully transduced with either AAV6-scramble miRNA-GFP or AAV6-Vtilb miRNA-GFP. Only $3 \%$ of the transduced neurons were positive for Vtilb in the case of AAV6-Vtilb miRNAGFP, whilst in the control no reduction of the Vtilb expression in GFP-positive cells was observed (Vti1b/GFP, Fig. 3.9 B). Interestingly, 28\% of all TRPV1-expressing neurons were also positive for GFP (GFP/TRPV1, Fig. 3.9 C). This did not differ between the scramble and Vtilb miRNA group (Fig. 3.9 C) revealing that the knockdown of Vti1b does not have a major effect on TRPV1 expression.

To assess changes in somatosensory behaviours upon downregulation of Vti1b, mice were tested prior to virus injection for (i) their basal mechanical pain threshold with the dynamic aethysiometer (withdrawal latency of the hind paw to a mechanical stimulation), and (ii) their thermal threshold with the Hargreaves test (withdrawal latency to a radiant heat stimulus). These behavioural tests were repeated 3 weeks after the injections and did not reveal any changes in the basal sensitivity upon knockdown of Vtilb (Fig. 3.9 D). Our in vitro calcium imaging data show that knockdown of Vti1b leaves normal/non-sensitized functioning of TRPV1 untouched as the responses to the initial, single capsaicin stimulus were not different to control siRNA. To investigate whether normal function of TRPV1 is also unchanged in vivo, I performed an intradermal injection of a single dose of capsaicin. WT mice show acute nocifensive behaviour (e.g. licking, shaking and flinching) immediately after the application of capsaicin, which is absent in Trpv1-KO mice (Caterina et al., 2000). Mice were treated with AAV6-Vtilb miRNA and scramble miRNA as described above and the total duration of nocifensive behaviour after intradermal capsaicin injection was recorded over a period of $6 \mathrm{~min}($ Fig. 3.9 E). As an additional control, a group 
of naïve WT mice were injected in parallel. The capsaicin-evoked nocifensive behaviour was similar between all 3 groups, suggesting the Vti1b-TRPV1 interaction is not relevant for the normal activity of TRPV1. On the other hand, the CFA-mediated sensitization was found to be compromised in vitro. Thus, intraplantar injection of CFA to induce inflammatory pain was performed in another cohort of AAV6-Vti1b miRNA/scramble miRNA-treated mice. Twenty-four hours post CFA the mice were tested for the development of thermal hyperalgesia (Fig. 3.9 F and Appendix Fig. 7.4). Both the control group and Vtilb miRNA mice exhibited a decrease in the withdrawal latency to a heat stimulus (hyperalgesia). However, the reduction in latency compared to the contralateral paw was significantly lower in the Vti1b miRNA group, indicating a reduced development of thermal hyperalgesia if Vtilb expression is downregulated. This supports the notion that Vtilb is pro-algesic and facilitates TRPV1 sensitization. In a next step, I investigated whether Vti1b might affect pain behaviours upon inflammation which are largely independent of TRPV1. I did not observe any differences in the development of mechanical allodynia $24 \mathrm{~h}$ and $48 \mathrm{~h}$ after the CFA-injection (Fig. 3.9 G and Appendix Fig. 7.4) for which TRPV1 is postulated to only contribute to a small degree (Caterina et al., 2000; Gavva et al., 2005; McGaraughty et al., 2008). These results lend partial support for a selective effect of Vti1b on TRPV1.

\section{Figure 3.9: AAV6-mediated knockdown of Vti1b reduces thermal hyperalgesia after inflammation}

(A, B) Co-immunostainings on IDRG of mice injected with AAV6-Vti1b miRNA-GFP (Vti1b miR, upper panels) and AAV6-scramble miRNA-GFP (Scramble miR, lower panels). (A) Representative images show successful knockdown of Vtilb (red) in GFP-positive cells (green) of the Vtilb miR group 3 weeks after virus injection. Exemplary GFPimmunoreactive neurons are labelled with white asterisks. Note that in the merged images of the scrambled miRNA most of the cells that show overlap of GFP and Vtilb do not appear completely yellow, as the Vtilb staining does not fill the complete cytoplasm. Scale bar, $50 \mu \mathrm{m}$. (B) Quantitation to determine the Vtilb knockdown. Percentage of neurons immunoreactive for GFP (GFP) did not differ significantly, but the percentage of Vtilb-positive (Vtilb, $* *$ p < 0.01) as well as the fraction of neurons immunopositive for Vtilb to GFP-positive ones (Vtilb/GFP, $* * * p<0.001$ ). Cell count per label in columns; in total $\mathrm{N}>2400$ neurons from 4 mice (3 $\mathrm{lDRG} /$ mouse) per condition analysed. Significance was determined with one-way ANOVA followed by Bonferroni's multiple comparison tests. (C) Quantitation of coimmunohistochemistry on IDRG sections from Vtilb miR and scrambled miR-injected mice (sacrificed after capsaicin treatment) labelled for TRPV1 and GFP. No significant differences were determined by one-way ANOVA; cell count per label in columns; in total N > 2000 neurons from 4 mice per condition (3 lDRG/mouse. (D) Knockdown of Vtilb does not change basal sensitivity to a radiant heat stimulus $(\mathbf{D}$, left) or punctuate mechanical pressure (D, right), as no change in the withdrawal latency of the ipsilateral paws was observed. Mice were tested prior to virus injection (pre AAV6) and 3 weeks after the injection (pre CFA). No significant differences were determined by Kruskal-Wallis test, $\mathrm{N}>10$ mice for each group, $n=2$ independent cohorts. (E) Capsaicin-induced nocifensive behaviour is unaffected by Vti1b knockdown. Mice injected with Vtilb miR or scramble miR and naïve WT mice received intradermal capsaicin injections $(0.5 \mu \mathrm{g})$. The time spent licking, shaking, flinching or lifting the treated hind paw was measured over a period of ten minutes. No significant differences were determined by Kruskal-Wallis test, $\mathrm{N}>6$ mice per condition, $\mathrm{n}=2$ independent cohorts. (F) Vti1b knockdown diminishes heat hyperalgesia after CFA-induced inflammation. Mice injected with Vti1b miR or scramble miR developed heat hyperalgesia $24 \mathrm{~h}$ after CFA injection. This is reflected in the reduction of the withdrawal latency to a radiant heat stimulus for the ipsilateral paw. The mice injected with Vtilb miR exhibited significantly less hyperalgesia, ${ }^{*} \mathrm{p}=0.0128, \mathrm{~N}=10$ per group, $\mathrm{n}=2$ independent cohorts, Mann Whitney test. (G) Vtilb knockdown does not affect mechanical hypersensitivity after CFA-induced inflammation. Mice injected with Vtilb miR or scramble miR developed mechanical hypersensitivity $24 \mathrm{~h}$ after CFA injection. This is reflected in the reduction of the withdrawal latency for the ipsilateral paw to a mechanical stimulus. No significant difference was observed between groups, $\mathrm{N}=8-10$ per group, $\mathrm{n}=2$ independent cohorts, Mann Whitney test. All Data represented as mean \pm SEM. D, F, G: withdrawal latencies for the contralateral paws as well as normalized values (\% to contralateral paws) can be found in Appendix Fig. 7.4. From Sondermann et al., 2019. 
A
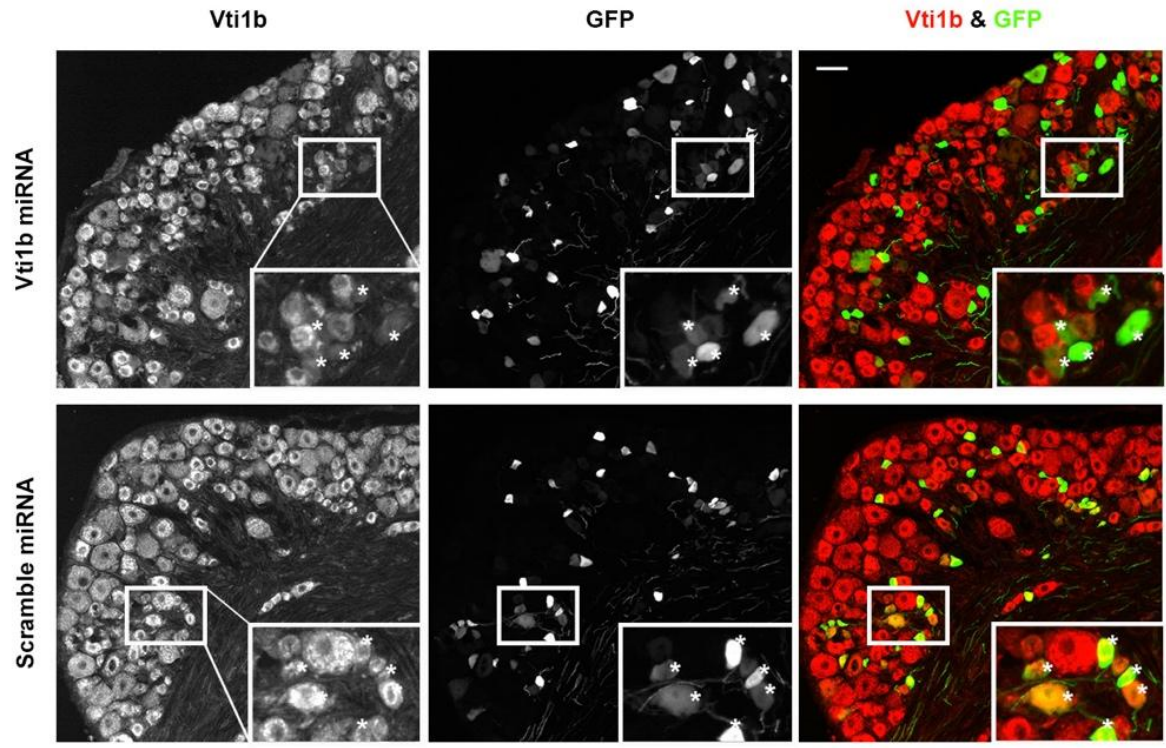

B

C
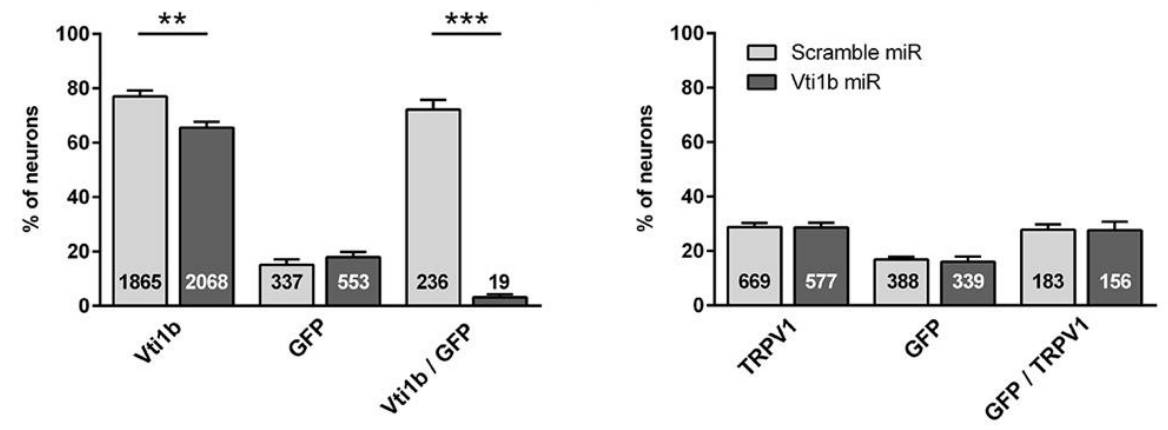

D
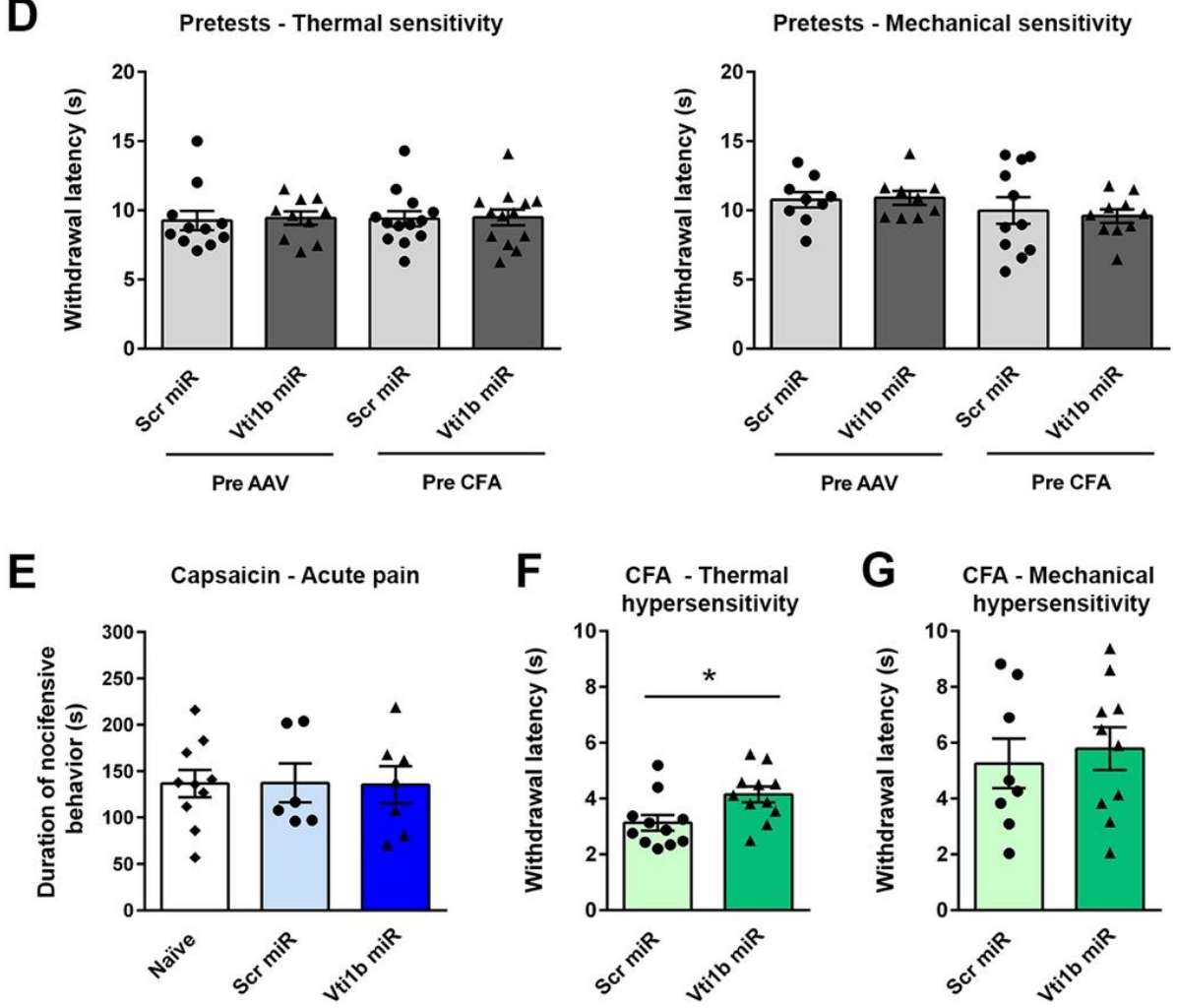


\subsubsection{Vtilb belongs to the TRPV1 interactome}

This section is based on Sondermann et al., 2019.

All data shown so far propose that Vti1b has a pro-algesic role in IDRG neurons by targeting TRPV1. Thus, one would assume that both proteins can be found near each other in a neuron to interact with each other. To test this hypothesis, I performed a Proximity Ligation Assay (PLA), a technique to investigate the close proximity of two proteins in situ. The procedure is similar to an ICC in that primary antibodies against the target proteins are applied. However, the 2 secondary probes have to be $\leq 40 \mathrm{~nm}$ apart from each other to be able to interact. This interaction then gives rise to a measurable signal (Söderberg et al., 2006). Fig. 3.10 A, middle panel shows a representative image of the PLA with antibodies against TRPV1 and Vtilb on cultured WT IDRG neurons. As control for the specificity of the PLA signal, the PLA was done on neurons from Trpvl-deficient mice. Fig. 3.10 A, right panel shows the fraction of cell area that was PLA-positive (\%area, i.e. the area showing PLA signal above threshold) per individual cell for WT and Trpv1-deficient DRG neurons. None of the Trpv1-deficient neurons showed PLA-positive signal for more than 7\% of their cell surface area, indicating that the increased PLA signal in WT neurons is specific for the Vtilb-TRPV1 interaction.

Furthermore, we performed PLA on HEK cells overexpressing TRPV1 either with Vti1b or the mock plasmid (Fig. 3.10 B). Signal quantification is presented in Fig. 3.10 B as cell area with PLA signal above threshold per individual cell. In TRPV1+ Vti1b expressing cells on average $50.8 \pm 3.6 \%$ of the cell area was PLA-positive, whilst in only TRPV1 expressing cells only $4.6 \pm 1.3 \%$ of the area was PLA-positive, indicating close proximity of the two proteins also in the heterologous expression system.

We wanted to further verify the co-localisation of the two proteins by a different method, thus, we performed co-immunoprecipitation (CoIP) against TRPV1 from HEK293 cells overexpressing Vti1b and TRPV1. Fig. 3.10 C shows the immunoblot of CoIPs from HEK cells transiently transfected with either both Vti1b-myc-DDK and TRPV1-YFP plasmids, or TRPV1-YFP and mock (empty pcDNA-Sport6 plasmid). Vtilb was successfully pulled down when co-expressed with TRPV1-YFP, suggesting a physical binding of the two proteins in a non-sensory cell context - however, not necessarily a direct binding, as the TRPV1-Vti1b interaction could be mediated by a third unknown protein. 
A
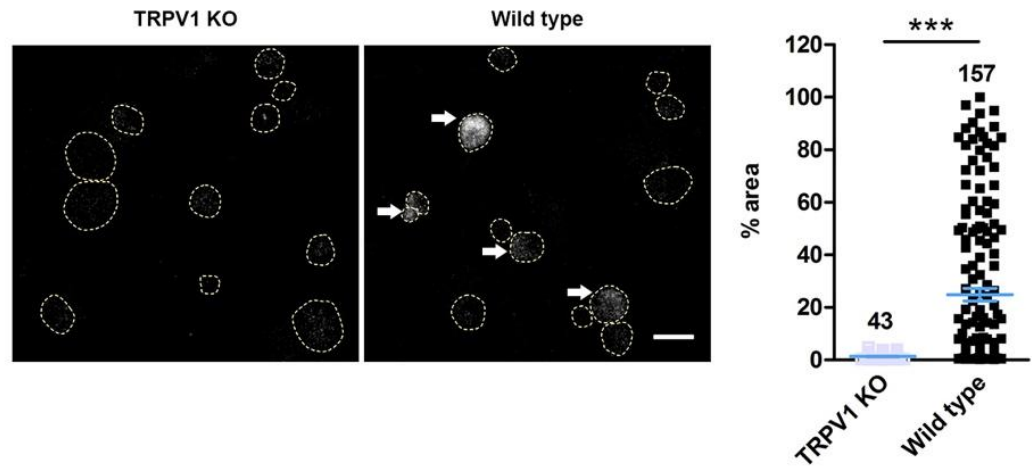

B
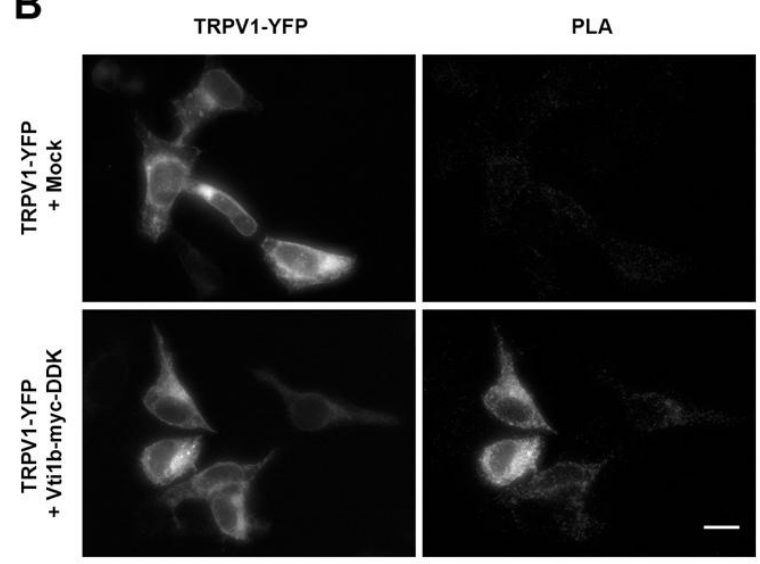

\section{C}
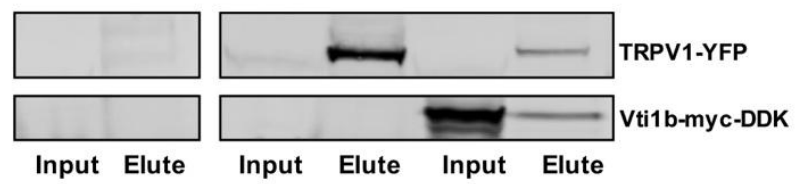

Mock

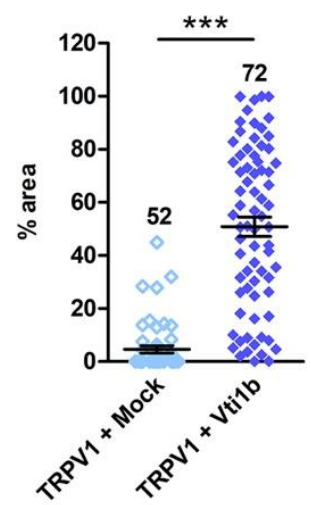

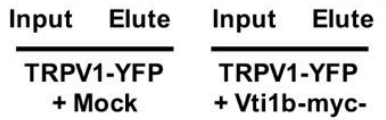

DDK

Figure 3.10: Vti1b is in close proximity to TRPV1 both in DRG neurons and upon heterologous expression

(A, left) Close proximity of Vti1b and TRPV1 is revealed by PLA with antibodies targeting TRPV1 and Vti1b in primary cultures of mice IDRG neurons of wild type mice as compared to neurons from TRPV1-KO mice that were treated similarly. Exemplary neurons that show PLA signal in $>20 \%$ of their cell area are marked with white arrows. Scale bar, $25 \mu \mathrm{m}$. (A, right) Sample quantification of (A, left). Results are displayed as \%area, ie. the \% of cell area with PLA signal above threshold per individual cell. Wild type vs. TRPV1 KO: $* * * \mathrm{p}<0.0001$, neuron count above scatter, $\mathrm{n}=2-3$ independent cultures, Mann-Whitney test. (B, left) Representative images of PLA with antibodies targeting myc (Vtilb) and TRPV1 on HEK cells that were transiently transfected with TRPV1-YFP + Vtilb-myc-DDK, or as control TRPV1-YFP + Mock. Scale bar, $15 \mu \mathrm{m}$. (B, right) Quantification of PLA signal on HEK cells (B, left). Results are displayed as \% area, i.e. the \% of cell area with PLA signal above threshold per individual cell (only YFP-positive cells were considered). TRPV1-YFP + Vtilb vs. TRPV1-YFP + Mock: *** $\mathrm{p}<0.0001$, neuron count above scatter, $\mathrm{n}=3$ independent cultures, Mann-Whitney test. (A, B) Data represented as mean \pm SEM. (C) Representative western blot of co-immunoprecipitation against TRPV1 from HEK cells transiently transfected with TRPV1-YFP + Vti1b-myc-DDK, TRPV1-YFP + Mock or only Mock $(n=5)$. Membranes were incubated with anti-TRPV1-Ab (upper panel) and anti-Vti1b-Ab (lower panel). TRPV1 was detected in the elute of both TRPV1 + Mock- and TRPV1 + Vti1b-transfected cells. Vtilb was detected only in the input and elute of the TRPV1 + Vti1b co-expressing cells. Of note, the lower, less intense band in the input of the TRPV1 + Vtilb-condition is an unspecific band. Appendix Fig. 7.5 displays the corresponding image of the whole immunoblot. From Sondermann et al., 2019. 


\subsubsection{Identification of pain-specific interactors of TRPV1 by quantitative mass spectrometry}

A part of this section is based on Sondermann et al., 2019.

Yet another method to identify interaction partners is functional proteomics - CoIP of the protein of interest followed by quantitative tandem mass spectrometry. We wanted to coimmunopurify native TRPV1 from murine IDRG to confirm that Vti1b is a member of the TRPV1 complex, but also go one step further and see whether we could detect a pain dependent regulation of the Vti1b-TRPV1 interaction as well as detect further members of the TRPV1 complex.

Inflammatory pain was induced by injecting CFA into the hind paw of wild type mice, which leads to a local swelling and inflammation of the paw accompanied by development of thermal and mechanical hypersensitivity. The mice were sacrificed $24 \mathrm{~h}$ after the injection and the IDRG innervating the inflamed paw were excised. After detergent-aided tissue lysis a CoIP was performed against the native TRPV1 channel. Subsequently, TRPV1 and copurified proteins were analysed and quantified by $\mathrm{UDMS}^{\mathrm{E}}$ (see section 2.1.7.2 for details). Since the idea was to profile the interactome dynamics between the pain condition and a physiological control, a second group of mice that were injected with the vehicle (Veh) was processed as described above. In addition, to reduce the list of false positive interactors due to unspecific binding to the affinity matrix, I performed the CoIP also from IDRG of Trpv1deficient (KO) mice. To take into account the biological variability as well as possible variances in sample preparation, 3 biological replicates of each condition (CFA, Veh, and $\mathrm{KO})$ were prepared and analysed.

In total 1089 proteins were identified and quantified in all 9 samples (see section 2.1.7.2 for details). For the bait TRPV1 34 unique peptides (54\% sequence coverage) were consistently detected in each of the 3 replicates of the Veh and CFA group. In the samples from the KO mice, serving as negative controls, TRPV1 was not detected. The amount of TRPV1 quantified across all replicates was not equal (see below).

To access the similarity of the replicates within and between the groups, the $\log 2$ transformed abundance values of all 9 samples were plotted against each other and the Pearson correlation coefficient was calculated (see Appendix Fig. 7.6). On average, the samples showed high correlation (0.681-0.968). As expected, the correlation within one group was higher than between the groups of one replicate round. Furthermore, the samples of the first replicate round showed a trend to lower correlation coefficients when being compared to the other replicates, round 2 and 3, respectively. All samples had been analysed on the same LC-MS platform, however, round 2 and 3, which were run consecutively, were acquired six months after round 1, indicating run-to-run differences in the acquisition.

Next, the detected amount of proteins in Veh or CFA, respectively, were compared to the $\mathrm{KO}$ group with regard to their fold change and the statistical significance of this enrichment.

Two issues had to be dealt with before a fair comparison could be carried out:

(I) Different amounts of the bait TRPV1 had been detected in the replicates of the CFA and Veh group, which could explain the observed differences in the detected amount of 
interactors between the conditions. Therefore, the amount of bait in CFA was divided by the amount in Veh (of the respective replicate round) and all proteins in the CFA group were normalized with this replicate-specific ratio.

(II) Many proteins had not been detected in 3/3 replicates and/or all conditions. Missing values pose a problem onto the statistical analysis using a t-test, which requires 3 valid values per group. One could simple omit the proteins not detected in $3 / 3$ replicates over all conditions, but this would most probably lead to the loss of weak or transient interactors, which might just not have crossed the detection limit of the mass spectrometer in one or the other run. Another well-established option, which is commonly used, is to impute the missing values, either by a constant value near the detection limit of the mass spectrometer, or by random values taken from a normal distribution simulating low abundance values (Hubner et al., 2010). I decided to try out both methods in parallel and compare the outcome. First, any protein that had not been detected in at least one group in 3/3 replicates was omitted (reducing the number of proteins to 1015), then missing values were imputed with

(a) a constant value of 5.41 ( $\log 2$ value of the lowest detected value over all runs, shifted down for one order of magnitude), and

(b) random values of a normal distribution from low abundant values (see Appendix Fig. 7.7) for distribution of imputed values and section 2.1.7.2 for further details on imputation). Second, I used a modified version of the Student's t-test (Tusher et al., 2001) to determine the difference between observed protein abundances in CFA / Veh and the KO control. In this version of the test an additional parameter called s0 is considered. In simple terms, one could understand the $\mathrm{s} 0$ as a minimal fold change that a protein must show next to a sufficient p-value to become a significant hit.

Proteins with q-values $<0.05$ and s0 $>1.58$ (equals a fold change of 3) were stated as significantly enriched and defined as putative interactors of TRPV1. Plotting the observed $\log 2$ fold change versus the negative logarithm of the p-value results in a "volcano plot" (Fig. 3.11 A, B), where putatively interesting candidates (i.e. high fold change \& high -log p-value) can be easily distinguished from background binders (Hubner et al., 2010). These proteins that bind unspecifically to the matrix accumulate around the zero-line (low fold change ratio). The fraction of proteins in the upper left quadrant (i.e. significantly enriched in the KO group) can also be discarded for the further analysis as binding completely unspecific to the affinity matrix.

In total 95 proteins for CFA and 115 for Veh showed an at least 3-fold enrichment to the KO control, when imputation of missing values was done with random values (b). Of these proteins, 55 were enriched in both CFA and Veh. On the contrary, if imputing with a constant value of 5.41, 127 proteins for CFA and 169 for Veh showed an enrichment and 89 proteins were enriched in both groups (Fig. 3.11 C).

The list of significantly enriched proteins (of imputation method (b)) was compared with entries of the TRIP Database 2.0 (http://www.trpchannel.org/summaries/TRPV1), an online databank containing previously reported interactors of mammalian TRP channels. Both TRPV2, a well-established interactor of TRPV1 (Hellwig et al., 2005) and the dynactin subunit 1 (Dctn1, Stein et al., 2006) were found previously by complementary methods. In 
addition, extended synaptotagmin-1 (Esyt1) was detected that had been found previously in the interactomics screen from Hanack et al. (Hanack et al., 2015).

Vtilb was detected in both Veh and CFA ( $\log 2$ fold change $\mathrm{Veh} / \mathrm{KO}=4.1 ; \mathrm{CFA} / \mathrm{KO}=4.43$, Fig. 3.11 A, B, \#3). Strikingly, the detected amount of Vtilb in CFA was approx. 20\% less than in Veh suggesting a decrease in the interaction with TRPV1 upon inflammatory pain. This is in line with our proteomics data (Barry et al., 2018; Rouwette et al., 2016).

Subsequently, to define the pain-specific interactors, the significantly enriched proteins of Veh and CFA were compared to each other. Supposing that a $20 \%$ regulation of the interaction might be biologically relevant (as demonstrated for Vtilb), I defined painspecific interactors among the proteins (significantly enriched compared to $\mathrm{KO}$ ) as having a $\log 2$ fold change of CFA/Veh $>0.263$ or $<-0.263$, respectively. This resulted in 50 (57) proteins that associate with TRPV1 only under inflammatory conditions, while 83 (126) dissociate (see Appendix Table 7-1).

The imputation of missing values with the constant value of 5.41 yielded overall more proteins that were counted as putative interactors of TRPV1. Most of the proteins that were not detected using the imputation with random values are low abundant ones $(\log 2$ abundance values ranging from 8-12) with the need of imputing 3 values for the KO control group (i.e. detected in $0 / 3$ replicates). Therefore, if one imputes with random values from a normal distribution simulating low abundant values (mostly values between 8-11), these do not cross the threshold for being considered significantly enriched compared to the KO group ( $\log 2$ fold change $>1.58$ ). Setting a low value like 5,41 avoids this problem as exemplary shown for Stx 8 (compare location of \#4 in Fig. 3.11 A, B and values in D). However, for cases in which only $1 / 3$ or $2 / 3$ values are missing, it seems more reasonable to impute with random low abundance values to avoid the artificial decrease in the group mean (if imputing with 5.41) that in case of Stx8 leads to defining the protein as "pain- specific" ( $\log 2$ fold change < or $>0.263)$, in contrast to not being detected above threshold to KO ( $\log 2$ fold change $<1.58)$ at all (Fig. 3.11 D).

Optimally, one would analyse the cases with 0/3 detections in the $\mathrm{KO}$ (and also for 0/3 in CFA or Veh) separately, without performing a t-test (see Appendix Table 7-2). However, they should not be attributed with a fold change (CFA/KO or Veh/KO or CFA/Veh) as one cannot be certain that the protein was really not present in the KO samples (abundance of 0 ), or maybe it was just below the detection limit in $3 / 3$ replicates of the KO group (unknown abundance value $>0$ ) and therefore, any calculated fold change would be erroneous.

For the final analysis of the interactomics data as published in Sondermann et al., 2019, we decided to use the imputation with random values from a normal distribution simulating low abundant values to exclude unsecure candidates like Stx8. Also, we set a more stringent threshold for significantly regulated proteins (q-value $<0.01$, compare Figure 6 and Materials and methods 2.19.2. in Sondermann et al., 2019) to further decrease the probability of false positives. 
A
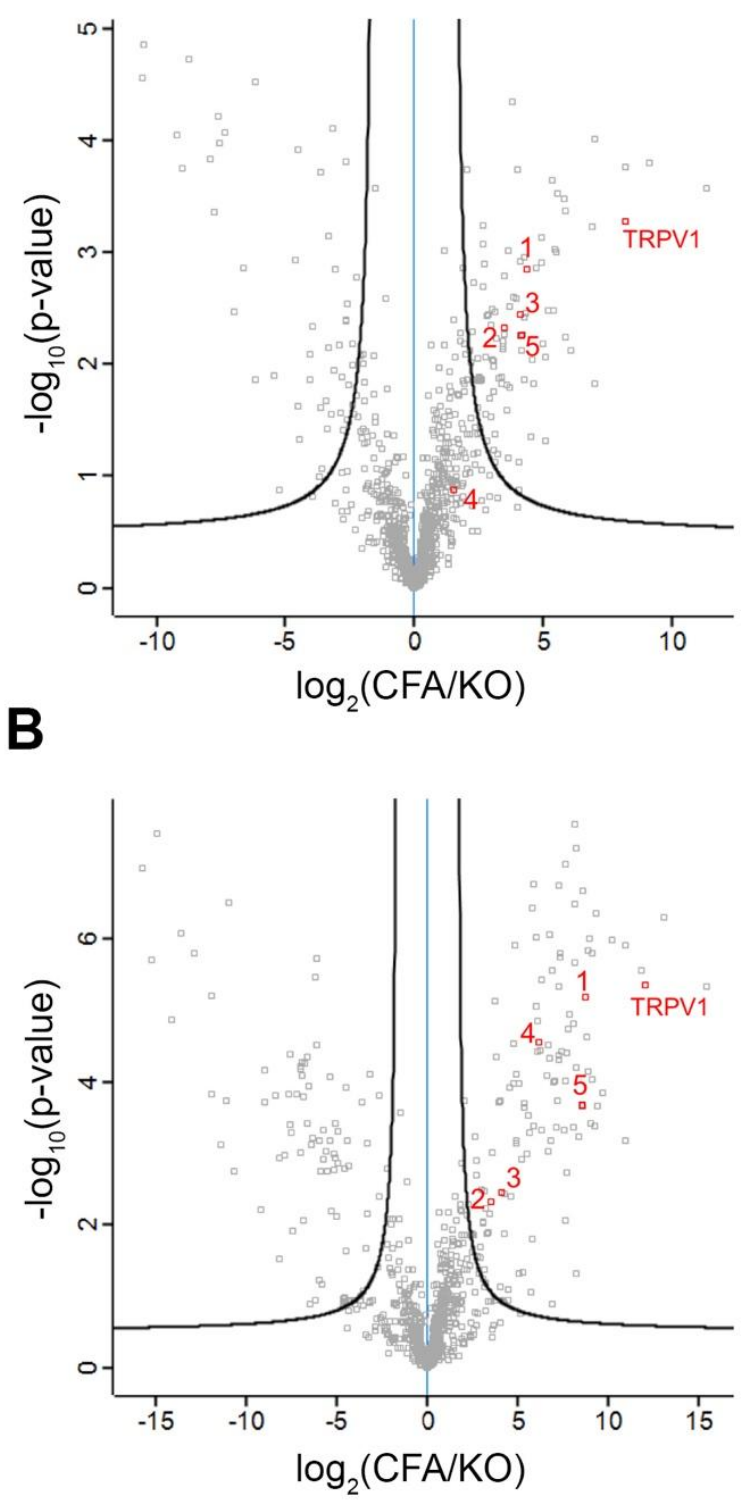
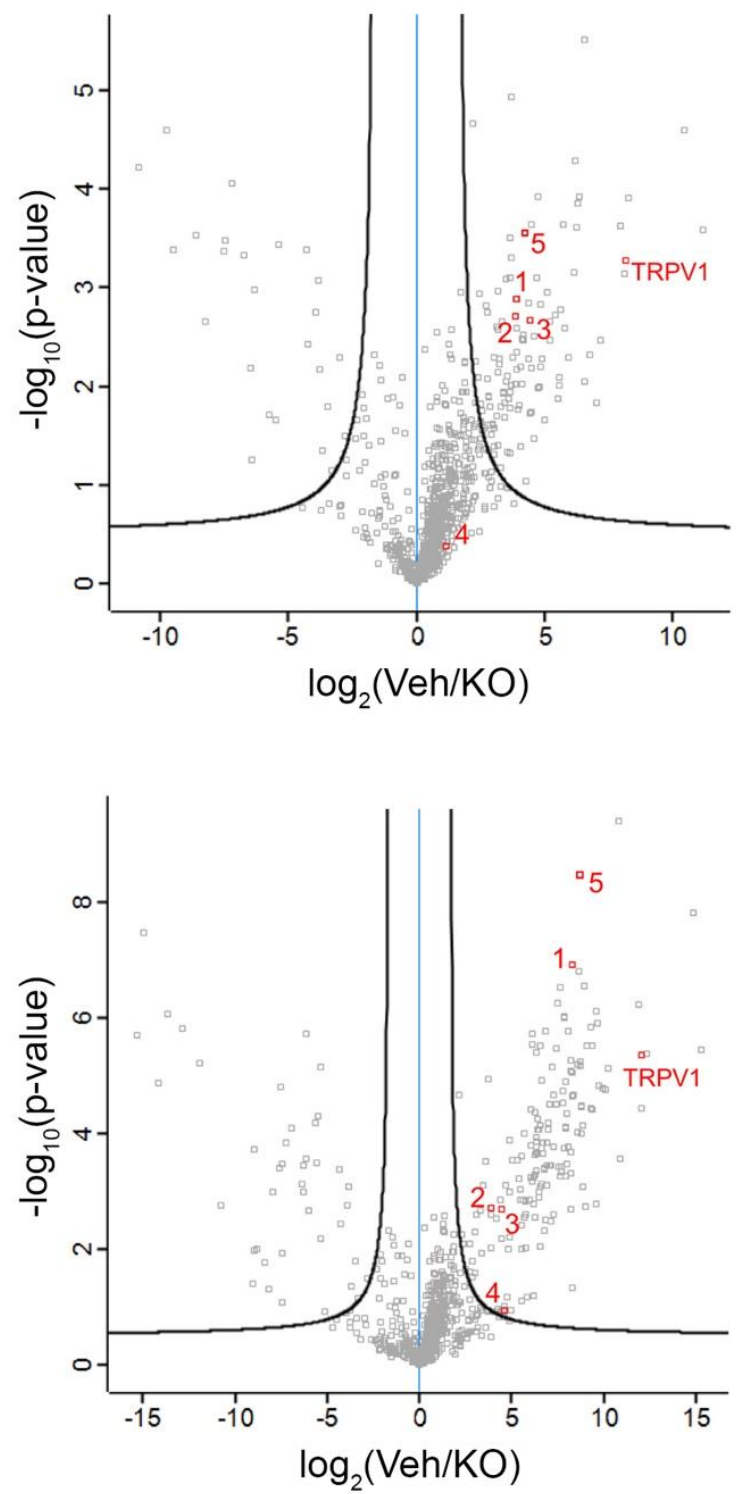

C

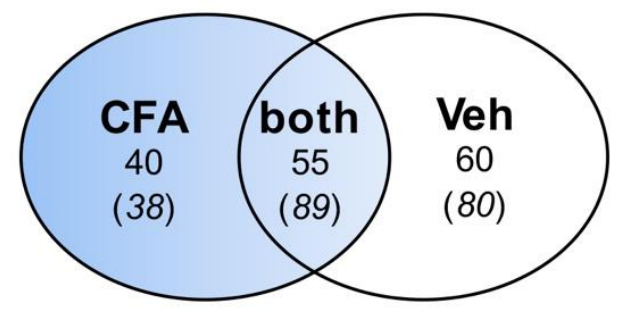

D

\begin{tabular}{|r|c|c|c|c|c|c|c|c|c|}
\hline & KO1 & KO2 & KO3 & CFA1 & CFA2 & CFA3 & Veh1 & Veh2 & Veh3 \\
\hline Log $_{2}$ abundance & $\mathrm{NaN}$ & $\mathrm{NaN}$ & $\mathrm{NaN}$ & 11.30 & 11.31 & 12.17 & $\mathrm{NaN}$ & 12.63 & 11.95 \\
\hline Random values & 8.56 & 10.99 & 10.66 & & & & 9.14 & & \\
\hline Constant value & 5.41 & 5.41 & 5.41 & & & & 5.41 & & \\
\hline
\end{tabular}

\begin{tabular}{|r|c|c|c|}
\hline $\log _{\mathbf{2}}$ (ratio) & Veh/KO & CFA/KO & CFA/Veh \\
\hline Random values & 1.17 & 1.52 & na \\
\hline Constant value & 4.59 & 6.18 & 1.60 \\
\hline
\end{tabular}


Figure 3.11: Determination of high confidence interacting proteins

(A, B) Volcano plots of Veh vs. KO, and CFA vs. KO: imputation of missing values was done with random values simulating low abundancy values in (A), and imputation done with constant value in (B). The $\log 2(\mathrm{Veh} / \mathrm{KO}$ or CFA/KO) ratio depicts the difference between the means of the 2 conditions in each t-test (reflecting the enrichment of proteins) and is plotted against the negative $\log \mathrm{p}$-value of the t-tests. The cut-off line is based on an estimation of the FDR by permutation. Proteins (on the right side of the vertical zero line) are considered as high confidence interactors when being right from the cut-off curve, while proteins with negative ratio or ratio close to zero are considered as unspecific binders. Numbers 1, 2, 5 represent examples of previously reported interactors of TRPV1: 1, TRPV2; 2, Dctn1; 3, Vti1b (see section 3.2.1); 4, Stx8 and 5, Esyt1. Number 4 highlights Stx8, as an example of how the fold change and significance of putative interactors depends on the chosen imputation method (compare D). (C) Venn diagram displaying the overlap of significantly enriched proteins in the CFA and Veh group. (D) Tables displays measured $\log 2$ abundance values for Stx8, imputed values for missing values and the calculated fold changes. Fold changes in the case of imputation with random values does not exceed the required minimal $\log 2$ fold change of 1.58 , therefore the protein is not considered for the comparison $\mathrm{CFA} / \mathrm{Veh}$ in that case. na, not applicable, NaN, not a number.

Using the Database for Annotation, Visualization and Integrated Discovery (DAVID), I investigated the biological implication behind the putative pain-specific interactors. Several clusters of GO annotation terms were found to be enriched, amongst others "intracellular protein transport" for the proteins dissociating from TRPV1 under inflammatory pain and "actin binding" for the ones associating with TRPV1 under inflammatory pain (Appendix Table 7-3 and 7-4). To identify predicted associations among the candidates, I uploaded the dataset to STRING and did a network analysis, reporting direct and/or functional interactions based on experimental validation and curated databases (Appendix Fig. 7.8 and 7.9). Yet, no extensive clusters of interactions were revealed.

To sum up, the functional relevance of one putative interactor of TRPV1, Vti1b, was assessed for TRPV1-mediated nociceptive signalling. These functional studies revealed differences in TRPV1-mediated neuronal responses to capsaicin. More specifically, Vtilb affects the desensitization behaviour of TRPV1-expressing sensory neurons upon repetitive capsaicin stimulation. In vivo local knockdown of Vtilb in IDRG did not affect basal nociceptive thresholds and TRPV1-dependent acute pain, but only the CFA-induced development of thermal hyperalgesia was diminished after $24 \mathrm{~h}$.

Furthermore, I applied a functional proteomics approach to identify the TRPV1 interactome under CFA-induced inflammatory pain in mouse DRG neurons. A significant regulation of the TRPV1 interactome upon induction of inflammatory pain was revealed. 


\section{Discussion}

\subsection{Insights into chronic pain by standardized DRG proteome profiling}

This section is based on Rouwette et al., 2016.

Standardized and reproducible detection and quantitation of the constituents of protein signalling networks is crucial for deciphering the molecular underpinnings of physiological and pathophysiological processes. Here, we present a multi-layer DIA-MS-based workflow that allows to detect and quantify alterations in the mouse DRG proteome in two chronic pain models in a standardized way.

DRG harbour the somatosensory neurons that detect both noxious and innocuous stimuli. These neurons undergo phenotypic changes under pathological conditions causing exaggerated transmission of pain messages to the brain, thereby, contributing to chronic pain symptoms like hypersensitivity and spontaneous pain (Basbaum et al., 2009). Therefore, studying the molecular changes underlying pathological pain states in DRG neurons is key for identifying potential novel drug targets. Interference with the first step of nociceptive signalling by local targeting of the DRG represents a promising alternative to the systemic application of drugs, or drugs targeting the CNS, especially with respect to adverse side effects (Patapoutian et al., 2009; Sapunar et al., 2012).

Matching our DIA-MS data with our self-generated spectral library, we reproducibly identified several dozens of proteins that showed significant and mostly pain model-specific up- or downregulation in the chosen pain paradigms. Since the DRG comprises several different cell types, we cannot confer the observed changes solely onto sensory neurons. Yet, we partially dealt with this problem by using in situ immuno-labelling of proteins and cell type-specific functional assays to relate the detected abundance changes to neurons.

We found several proteins previously reported to be relevant for painful pathologies in vertebrates with our DIA-MS-based workflow. This demonstrated that we were able to obtain meaningful and interesting candidates. Approximately $50 \%$ of all regulated proteins had not been related to somatosensation and pain before and among these are some scarcely characterized ones, which represent an especially interesting subgroup of candidates for further investigations on their role in nociception and pathological pain.

Further insight into the molecular signature of chronic pain can be gained by zooming out from single regulated proteins and looking at the interdependencies between many of them, ultimately creating cellular signalling networks. The consequences of dysregulation of major signalling pathways have already been implicated in chronic neuropathic pain, for instance, mitochondrial dysfunction (Baloh, 2008; Fernyhough et al., 2010; Joseph and Levine, 2006) and disturbances of protein biosynthesis (Inceoglu et al., 2015; Lupachyk et al., 2013; Zhang et al., 2015). Given the complexity of these protein networks, the limited use of classical interventions targeting single proteins for analgesia become evident. Thus, emerging "network medicine" strategies are aimed at correcting functional misalignments of cellular processes (Barabási et al., 2011; Borsook et al., 2014; Schapira, 2012). Interference on the network level was shown to be successful for several animal studies (Inceoglu et al., 2015; 
Joseph and Levine, 2006; Lupachyk et al., 2013) and might provide promising tools for achieving analgesia. Analysing our significantly regulated proteins with STRING, we report abundance changes of dozens of proteins belonging to complexes of the mitochondrion and proteins important for protein biosynthesis and maturation in the ER for both chronic pain models. In order to validate the in vivo relevance of these networks in inflammatory pain, we applied inhibitors of either the mitochondrial ETC-complex I or PDIs. With both inhibitors we achieved acute analgesic effects in fully-established persistent inflammatory pain. These data support and complement previous studies linking dysfunction of the mitochondrial respiratory chain and ER stress to painful peripheral neuropathies (Inceoglu et al., 2015; Joseph and Levine, 2006; Lupachyk et al., 2013). We could demonstrate the relevance of these signalling network also in persistent inflammatory pain induced by CFAinjection and moreover, identifying regulated members of these networks. These could therefore represent putative targets for novel analgesic treatments.

However, our data did not confirm previously reported regulations of several nociceptive ion channels under pathological pain. The nocisensor TRPA1, for example, was reported to be upregulated under CFA-evoked inflammatory pain (da Costa et al., 2010), however, our data do not support a significant regulation. A number of technical factors could account for this and further discrepancies to other studies. We (and others using MS techniques) not only used the IDRG 3-5 that contribute the major innervation of the hind paw, but pooled them with IDRG $1+2$ to have enough tissue for our sample preparation and the following mass spectrometry. But even within one DRG some neurons may be strongly affected, whilst others are only weakly affected by the treatment, as this depends on whether the painevoking agents spread into the innervation field/receptive fields of the neurons in the periphery (Laedermann et al., 2014).

Other parameters to consider when comparing our data to previous proteomic studies profiling chronic pain are the species differences and the pain model used (Laedermann et al., 2014), the time point of tissue isolation and the biochemical sample preparation. As mentioned before membrane proteins are essential for pain signalling, yet, typically underrepresented in MS studies (Helbig et al., 2010). We therefore decided to prepare a membrane-enriched fraction to facilitate the MS detection of membrane proteins, by that we depleted many cytosolic proteins. This could explain the marginal overlap with previous MS studies, most of them analysing whole cell lysates (Melemedjian et al., 2013; Niederberger and Geisslinger, 2008; Vacca et al., 2014; Zou et al., 2012).

The use of a reference spectral library in DIA-MS enables the unambiguous identification and reliable quantification of 1000 s of proteins across many samples, yet, if a peptide signature is not stored in the applied library, the belonging protein cannot be detected. Our customized library contains the peptide signatures of several ion channels, such as TRPV1 and TRPA1, which are crucially implicated in the development of pathological pain and therefore, represent prime targets for the development of novel analgesics (Patapoutian et al., 2009). Unfortunately, the library lacks the signatures of the voltage-gated sodium channel $\mathrm{Na}_{\mathrm{v}} 1.7$, mutations of which confer congenital insensitivity to pain in humans (Cox et al., 2006), however, related and also nociceptor-specific $\mathrm{Na}_{v}$ - subtypes $\mathrm{Na}_{v} 1.8$ and $\mathrm{Na}_{\mathrm{v}} 1.9$ 
(Waxman and Zamponi, 2014) were detected. A reason could be insufficient solubilisation combined with an overall low expression of the $\mathrm{Na}_{\mathrm{v}} 1.7$ in the DRG, so that even with our membrane enrichment, we did not yield enough peptides for identification by MS.

\subsubsection{Conclusion and outlook}

We successfully applied emerging DIA-MS to reveal significant proteome changes expanding our knowledge about the molecular mechanisms of chronic pain conditions.

Our spectral library, which contains the information required for the standardized identification and quantification of 100s of membrane-associated DRG proteins, constitutes a unique resource for the further research on these proteins in any mouse model of interest. Furthermore, our DIA-MS dataset can be re-analysed in silico using an updated version of the spectral library extended with previously not detected proteins (see (Barry et al., 2018)). In this line, my host laboratory put efforts in extending the profiling onto different stations along the pain signalling pathway - from the periphery up to the brain - as well as for several further pain models. This should facilitate the comprehensive characterization of the distinct molecular underpinnings of well-defined pain syndromes. Ultimately, these efforts could help for the identification of putative drug targets as well as of biomarkers for chronic pain syndromes to allow for a better diagnosis. 


\subsection{Identification and characterization of TRPV1 protein complexes}

A large part of section 4.2 is based on Sondermann et al., 2019.

Ion channels are embedded in a network of proteins modulating their localisation and function. Stable protein-protein interactions (PPIs) are required for their general function but some of them, mostly of transient nature, can modulate the channels' properties as required in response to changes in the cellular status or environment (Schulte et al., 2011).

The TRPV1 channel has been shown to interact with different proteins that modulate its responsiveness to stimuli as well as its trafficking to and from the plasma membrane. Thereby the sensitization of nociceptive sensory neurons can be regulated. Here, Vtilb was identified as a novel interactor of TRPV1. Vti1b influences the sensitization of TRPV1 in an inflammatory pain condition. In primary cultures of sensory neurons inflammation-induced sensitization of TRPV1 was facilitated by Vti1b during repetitive stimulation. Yet, Vti1b did not affect the normal functioning/gating of TRPV1. Remarkably, only the development of inflammation-evoked thermal hypersensitivity was reduced in mice in which Vtilb expression was downregulated in IDRG.

Little is known about the dynamics of the TRPV1 interactome during different pain states. To examine whether the TRPV1-Vtilb interaction is differentially regulated between an inflammatory pain and a physiological condition, I employed a functional proteomics approach. To this end, I induced inflammatory pain in mice by injecting CFA and combined affinity-based co-purification of native TRPV1 and associated proteins from DRG with highly sensitive quantitative mass spectrometry. Vehicle (Veh)-injected wild type mice and Trpv1-KO (KO) mice served as controls. With this I confirmed the Vti1b-TRPV1 interaction and showed that under inflammatory conditions less Vtilb is detected within the TRPV1 complex. Furthermore, I shed light on the protein scaffold interacting with TRPV1 under patho-/physiological conditions. I found that the TRPV1 interactome undergoes pronounced changes upon induction of inflammatory pain.

\subsubsection{Validation of the Vtilb-TRPV1 interaction in vitro}

The sensitization of TRPV1 by inflammatory mediators is in part responsible for the enhanced responsiveness of nociceptors under pathological conditions. One of several causes of this sensitization is an increased insertion of TRPV1 into the plasma membrane (Camprubí-Robles et al., 2009; Ferrandiz-Huertas et al., 2014; Ji et al., 2002; MorenillaPalao et al., 2004; Zhang et al., 2005). Interestingly, this regulated exocytosis has been shown to be at least in part dependent on SNARE proteins, as it can be opposed by a peptide interfering with the action of the SNARE protein SNAP-25 (Camprubí-Robles et al., 2009). Furthermore, the vesicular proteins Snapin and synaptotagmin IX were reported to interact with the N-term of TRPV1 and to mediate the rapid delivery of TRPV1 to the plasma membrane as response to PKC phosphorylation. In this context it should be noted that Vtilb has been suggested to be a rate-limiting factor for the stimulated exocytosis of the proinflammatory cytokine TNF $\alpha$ in macrophages (Murray et al., 2005), and to be involved in the secretion of cytolytic proteins by cytotoxic T-cells after antigen stimulation (Dressel et al., 2010). 
The calcium imaging data show that in sensory neurons Vtilb is not required for translocation of TRPV1 to the plasma membrane prior to any stimulation. Nor does it influence TRPV1's activation, as there was no significant variation in the response to the initial first pulse of capsaicin. Yet, this is in contrary to what I observed for the first pulse in HEK cells overexpressing TRPV1 and Vtilb. This controversy could be attributed to some generalized artificial effects of the overexpression of both TRPV1 and Vtilb that lead to disturbance of protein trafficking in HEK. Overexpression of (tagged) proteins is known to cause mislocalisation (Gibson et al., 2013). Also, Vtilb could have different interactors and consequently, fulfil different tasks in non-neuronal vs. neuronal cells.

In addition, HEK cells overexpressing TRPV1 and Vtilb showed less tachyphylaxis to repetitive capsaicin stimulation, i.e. the TRPV1-dependent calcium influx was maintained over 6 pulses of capsaicin. Sensory neurons derived from Veh-treated mice displayed the expected tachyphylaxis after repetitive capsaicin stimulation regardless whether Vti1b expression was downregulated via siRNA. In the CFA condition however, I observed a CFAinduced sensitization, i.e. less tachyphylaxis. Yet, when Vti1b was downregulated, less TRPV1-expressing neurons were sensitized, i.e. fewer responded to the $6^{\text {th }}$ pulse of capsaicin. One explanation could be less functional TRPV1 residing at the membrane upon Vti1b knockdown as compared to control. Unfortunately, up to now there is no commercially available antibody that binds to the extracellular parts of the mouse TRPV1 channel. Also, experiments with surface biotinylation to determine differences in TRPV1 levels at the plasma membrane were not successful (data not shown).

If the diminished CFA-evoked sensitization is based on less functional TRPV1 residing at the plasma membrane after repetitive stimulation, two opposing mechanisms are possible: (i) Vtilb could promote a rapid replenishment of functional TRPV1 at the membrane after capsaicin-induced internalization, or (ii) it could prevent the internalization of TRPV1 from the membrane after capsaicin stimulation. Little is known about the regulation of the membrane content of TRPV1 via endocytosis (Ferrandiz-Huertas et al., 2014). One study by Holland et al. found that the sensory neuron-specific knockout of the E3 ubiquitin ligase MYCBP2 prevents capsaicin-induced desensitization of sensory neurons via prevention of internalization of TRPV1 (Holland et al., 2011). They found that the MYCBP2-KO leads to a constitutive activation of $\mathrm{p} 38$ MAP kinase pathway, a pathway known to sensitize TRPV1expressing neurons, yet, it is unknown how the p38 activation leads to inhibition of endocytosis (Holland et al., 2011).

Of course, also other mechanisms known to modulate TRPV1 de-/sensitization like gating properties such as open probability could be affected. We tried to apply the same 6-pulse stimulation protocol during electrophysiological recordings but were not able to acquire stable traces. Therefore, and due to the well-established role of Vtilb in trafficking processes, we decided to continue with calcium imaging and block the internalization of TRPV1 from the plasma membrane. Thereby the amount of functional TRPV1 at the membrane would only be regulated by the biosynthetic/secretory pathway (and of course, trafficking-independent desensitization mechanism). Application of dynasore to prevent internalization of TRPV1 via clathrin-mediated endocytosis between the $5^{\text {th }}$ and $6^{\text {th }}$ pulse, recovered the desensitization in the Vtilb knockdown to the extent of the control condition of the CFA group. This supports a role for Vtilb in the activity-dependent trafficking of 
TRPV1. However, we cannot tell from this experiment at which step of the trafficking Vti1b comes into play. With dynasore the very first step of the endocytic pathway is inhibited, namely, the scission of clathrin-coated pits from the membrane, therefore, any step later on could potentially involve Vti1b. Membrane proteins that undergo endocytosis either follow the recycling pathway, i.e. retrograde transport to the trans-Golgi network, or they are degraded via multi-vesicular bodies/late endosomes (LE). Different SNARE proteins are suggested to mediate membrane fusion at all these trafficking steps (Chen and Scheller, 2001). The SNARE Vtilb was shown to be involved in both LE-LE fusion (Antonin et al., 2000) and fusion of LE with lysosomes (Offenhäuser et al., 2011). In line with this, I detected a partial co-localisation of Vtilb with LE and lysosomes in DRG neurons, which for other cell types has been reported previously (Kreykenbohm et al., 2002; Offenhäuser et al., 2011). Sanz-Salvador et al. reported that the prolonged exposure of capsaicin (for 20 min) triggered an initial rapid endocytosis of TRPV1 with subsequent targeting of the channel for lysosomal degradation in DRG neurons (Sanz-Salvador et al., 2012). However, since the effect we observed is very immediate, it is rather unlikely that within the short duration of our protocol TRPV1 is already being transported to and degraded by lysosomes.

\subsubsection{In vivo validation of the Vtilb-TRPV1 interaction}

Given the apparent functional relevance of the Vti1b-TRPV1 interaction for TRPV1mediated nociceptive signalling in vitro, the next question was whether modulation of the Vtilb expression in vivo has consequences on pain behaviour in mice.

To this end, I silenced Vti1b expression in IDRG of mice. The local Vtilb knockdown did not result in changes of the basal thresholds for mechanical and thermal stimuli. In addition, the Vti1b knockdown in vivo did not perturb nocifensive behaviour evoked by a single dose of capsaicin, which solely relies on TRPV1-activation (Caterina et al., 2000). These results are in line with my calcium imaging data showing that normal functioning under physiological conditions stays intact and only CFA-mediated TRPV1 sensitization is affected.

To see whether CFA-evoked sensitization is also altered in vivo, the mice with Vtilb knockdown (or control) received an intraplantar CFA injection and were tested for thermal hypersensitivity after $24 \mathrm{~h}$. The mice with Vtilb knockdown showed a diminished development of thermal hyperalgesia compared to the control group, $24 \mathrm{~h}$ after induction of inflammatory pain. Even though Trpv1-KO mice show profound deficits in development of heat hyperalgesia after inflammation, also other channels crucially contribute to CFAinduced thermal hyperalgesia after inflammation, for example, TRPM3 (Vriens et al., 2011). Therefore, I cannot claim that the anti-nociceptive effect of the Vti1b knockdown is solely due to the disruption of the Vtilb-TRPV1 interaction, but also other nociceptive channels might be affected. However, the test for mechanical allodynia $24 \mathrm{~h}$ and $48 \mathrm{~h}$ after CFAinjection did not show differences in the pain behaviour between the groups. Initially TRPV1 was proposed to not contribute to mechanical hypersensitivity after inflammation (Caterina et al., 2000), subsequent studies with TRPV1 antagonists as well as acute knockdown via shRNA against TRPV1 supported a role for TRPV1 in the development of mechanical 
hypersensitivity, yet, to a smaller degree than thermal hypersensitivity (Kanai et al., 2007; Szabo, 2005). Thus, obtained results argue for a rather TRPV1-specific effect.

On the other hand, it could be that the knockdown of Vtilb acts indirectly via modulation of the initial inflammatory processes such as the secretion of pro-inflammatory mediators on the development of hypersensitivity (compare Murray et al., 2005). This could be investigated by measuring the level of secreted inflammatory mediators, or by assessing the expression of certain receptors crucial for the inflammatory response in DRG (Lin et al., 2011).

Regarding the question how Vti1b might affect TRPV1-mediated nociceptive signalling, it should also be noted that one SNARE complex partner of Vti1b was detected to interact with TRPV1, too. Stx 8 - which amongst others can make up a SNARE complex with Vti1b, Stx7 and Vamp8 (Antonin et al., 2000) - has been found to increase the cell surface localisation of the TrkA receptor (Chen et al., 2014). TrkA receptors are responsive to NGF and thus, their activation is involved in the sensitization of nociceptors (Basbaum et al., 2009). Consequently, shRNA-mediated knockdown of Stx 8 resulted in analgesia in the formalin model for inflammatory pain (Chen et al., 2014). In my MS screen Stx8 was not detected in the $\mathrm{KO}$ at all and when I only compare CFA and Veh without imputing missing values for the KO, Stx8's interaction with TRPV1 is suggested to be significantly stronger in the pain condition. It is not known whether overexpression of Stx8 enhances pain in vivo. Interestingly, the global knockout of Vti1b leads to an increased degradation of Stx8 in several tissues (PNS tissue was not tested; (Atlashkin et al., 2003)). Therefore, it is tempting to speculate that the detected decrease of Vtilb expression in the DRG overall (Rouwette et al., 2016), and the decrease in the interaction with TRPV1 upon CFA might be an attempt of the body to counterbalance putative pro-algesic effects of a Stx8-Vti1b-TRPV1 interaction: When downregulating Vti1b in vivo, also the amount of Stx8 decreased, and consequently, I observed the analgesic effect. Thus, it would be interesting to assess whether the amount of Stx8 is changed upon AAV6-Vti1b miRNA-mediated knockdown in vivo. Depending on the results it could be worth examining the effect of a double knockdown of Stx8 and Vti1b.

\subsubsection{Quantitative MS to identify TRPV1 pain-specific interactors}

Several methods can be used to elucidate PPIs. Functional proteomics has become the method of choice as it enables the detection of PPIs in their native cellular environment. Furthermore, it is not restricted to only detecting binary PPIs as it is the case with the yeast2-hybrid system (Schulte et al., 2011). Several crucial interaction partners of ion channels expressed in the CNS have been elucidated by this means (Nadal et al., 2003; Schulte et al., 2006; Schwenk et al., 2009). Recently, Hanack et al. discovered the interaction of GABAB1 receptor subunit and TRPV1 by functional proteomics (Hanack et al., 2015). They pulled down TRPV1 from DRG of knockin mice overexpressing a tagged version of the channel. Strikingly, the described interaction only takes place under pathological conditions, when TRPV1 is sensitized (Hanack et al., 2015). 
With my functional proteomics experiment I confirmed Vti1b as a member of the TRPV1 protein complex and showed that upon inflammatory pain the amount of Vtilb is diminished in the TRPV1 complex. This is in line with our DIA-MS screen where Vtilb showed a downregulation in the CFA group ( $\log 2$ fold change $=-0.158$, multiple testing-corrected qvalue $=0.005$ (Barry et al., 2018))

Moreover, the idea of my functional proteomics experiment was to directly compare the interaction partners of TRPV1 between a pathophysiological and physiological state. The results reported in section 3.1/Rouwette et al., 2016 and further large-scale studies profiling the overall change in protein expression in different PNS tissues upon induction of pain (Barry et al., 2018; Huang et al., 2008b; Vacca et al., 2014) have shown that indeed it is possible to detect meaningful protein network changes between physiological and pathophysiological states with MS. Also, alterations of protein interactions between different cellular states were successfully detected by functional proteomics. For instance, Gerold et al. found that upon binding of hepatitis C virus to its host receptor CD81 (expressed on hepatocytes), a certain set of proteins in the target cell associate with the receptor in order to promote virus entry (Gerold et al., 2015).

The high sensitivity of modern mass spectrometers allows for single-step enrichment of the bait, and to omit lengthy purification protocols which not only entail a loss of sample but also negatively affect transient and weak interactions. Yet, the sensitivity comes at the price of detecting a lot of unspecific proteins binding to the affinity matrix that have to be filtered out. In order to efficiently eliminate these background binders, negative controls are used, i.e. pulldowns from knockout tissue (Schulte et al., 2011). For the subsequent filtration step the fold change, i.e. ratio of abundance of a protein in experimental condition vs. KO condition, can combined with calculating a significance score for each interaction (Hubner et al., 2010). Applying a standard t-test requires that the experiment be done at least in triplicates and in addition, proteins should have been detected in all three runs. Yet, especially for low abundance proteins it is common that missing values occur across the runs, which need to be imputed to allow for statistical comparison (Nesvizhskii, 2012).

As exemplified with the case of Stx8 in my dataset, the chosen method for imputation either a constant value, or random values taken from a normal distribution simulating low abundance values - can have a profound effect on the subsequent data analysis. One could divide the dataset prior to imputation into 2 subcategories: (i) proteins with only random, isolated values missing across the replicates in my 3 groups - due to run-to-run differences or inevitable differences in sample preparation - and (ii) proteins that were not detected in any of the replicates in one group making a random missing rather unlikely. For (i) the imputation with random values simulating low abundance values near the detection limit of the mass spectrometer seems more reasonable than a constant low value (as seen for Stx8). For (ii) the question is whether to perform statistical tests and calculation of a fold change at all for the first step of comparison (CFA/KO and $\mathrm{Veh} / \mathrm{KO}$ ) if the protein was not detected at all in the KO. The imputation with random low abundance values would lead to an erroneous low fold change assuming the protein was really absent from the samples of one group. But in case of low abundant proteins that might not have crossed the detection limit in all 
repetitions of the KO group by chance, imputing with a "0" or any constant value below the detection limit, would yield erroneous high fold changes. Overall, to not miss potentially pain-specific candidates, these "only CFA" and "only Veh" proteins should be submitted to the second step of analysis, i.e. CFA/Veh, yet, with reservations if they exhibit a low abundance in CFA and Veh.

In order to discriminate high confidence interactors from false positive hits, there are no universal cut-off parameters that work for each and every interactomics dataset (Hubner and Mann, 2011). Previously described interactions could give guidance for where to set the cut off, and in case of simple organisms studied in detail, such as yeast, this might be feasible (Keilhauer et al., 2015), but what if information about interactors is missing, or the known ones are not detected?

I only found the TRPV2, a channel which is reported to form heteromers with TRPV1subunits (Helliwell et al., 1998), dynactin subunit 1, an interactor that so far only showed up in a yeast-2-hybrid screen (Stein et al., 2006) and extended synaptotagmin-1 reported to be a putative interactor by Hanack et al. (Hanack et al., 2015). Neither did I detect the GABAB1

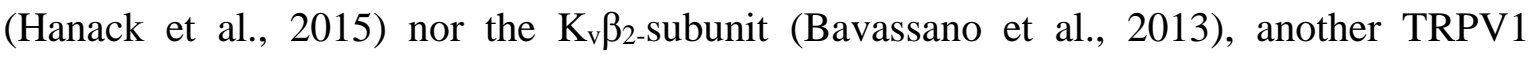
interactor proposed by a functional proteomics experiment. Also, these two studies detect only very few previously described TRPV1 interactors. Yet, one should keep in mind that the interactome detected by MS for a certain experiment can only represent a fraction of the complete and dynamic interactome of TRPV1.

Furthermore, several obvious differences in the study design could account for the missing overlap: Firstly, Hanack et al. used tissue from mice that overexpressed a tagged version of the channel and even though they proved the functional integrity of the overexpressed channel (Hanack et al., 2015), overexpression and the tag might disturb the "physiological" network of proteins interacting with the native channel. In addition, they did not use a stringent KO control but rather used wild type mice as controls. Secondly, differences in sample preparations must be taken into consideration that could crucially affect the fraction of TRPV1 interactome that is detected (Helbig et al., 2010; Schulte et al., 2011), for example, the amount of detergent used to solubilize the transmembrane protein TRPV1 varies. Detergents can differ greatly in their nature and strength, yet all of them inevitable affect PPIs. Thus, depending on the amount and kind of detergent used, different sets of PPIs are preserved, whilst especially dynamic interaction partners might be lost. Further, any kind of sample prefractionation steps, e.g. for enrichment of membrane fractions as done by Hanack et al., affects PPIs (Helbig et al., 2010). The use of antibodies against different epitopes of TRPV1 for the co-purification step could also explain the missing overlap/differences in the suggested interactors (Schulte et al., 2011). Thirdly, the MS platform used for analysis (instrument type, DDA vs. DIA) of the samples differs substantially and could explain for the detection of different subfractions of the TRPV1 interactome.

Yet, despite the missing overlap with previously described TRPV1 interactors, the follow up work of Bavassano et al. $\left(\mathrm{K}_{\mathrm{v}} \beta_{2}\right)$ and especially Hanack et al. $\left(\mathrm{GABA}_{\mathrm{B} 1}\right)$ as well as in my case the foregoing validation of Vtilb illustrate that the chosen functional proteomics approach was able to detect meaningful TRPV1 interactors. 
In order to gain more information about what kind of proteins had been detected by my interactomics screen, I looked for enriched cellular functions within the putative TRPV1 interactors. For the proteins significantly enriched in the CFA condition/inflammatory pain, an enrichment of actin-binding proteins was observed. Structural plasticity of neurons in the PNS and CNS - involving remodelling of both the actin and microtubule cytoskeleton - is suggested to be important for the transition from acute to chronic pain (Kuner and Flor, 2016). Increased peripheral target innervations have been reported to underlie hypersensitivity associated with different types of pathological pain (Costigan et al., 2009). Also, under CFA-induced persistent inflammatory pain cutaneous sensory hyperinnervation is observed (Chakrabarty et al., 2013). The increased axonal outgrowth was dependent on Angiotensin II and correlated with thermal hypersensitivity in established inflammatory pain, as pharmacological blockade of the Angiotensin 2 receptor (which is expressed by nociceptors) not only reduced the hyperinnervation several days after CFA injection, but also reversed the accompanied thermal hypersensitivity in rats (Chakrabarty et al., 2013).

Among the proteins significantly enriched in the Veh group, a clear enrichment of proteins connected to annotation terms related to "intracellular protein transport" was found. The rate of endo- and exocytosis determines the amount of TRP ion channels at the plasma membrane, thereby regulating their activity (Planells-Cases and Ferrer-Montiel, 2007) . As discussed above, the sensitizing action of several inflammatory mediators on TRPV1 has been shown to be mediated by stimulating the SNARE-dependent exocytosis of TRPV1 (Camprubí-Robles et al., 2009; Mathivanan et al., 2016; Morenilla-Palao et al., 2004). Also, Vti1b belongs to this group of proteins.

\subsubsection{Conclusion and outlook}

I validated the functional relevance of the Vti1b-TRPV1 interaction for TRPV1-mediated nociceptive signalling in vitro and more importantly, for pain behaviour in mice.

Still, there are unanswered questions in respect of the exact mechanism underlying the interaction and whether the analgesic effect of the Vtilb knockdown in vivo relies only on a direct effect on TRPV1. Pharmacological blockade of different steps of intracellular protein transport in vitro as well as high-resolution fluorescence microscopy with markers of subcellular compartments could help to clearly define the role Vtilb for TRPV1 trafficking/degradation.

Unfortunately, one cannot use Trpv1-KO mice to determine that the observed analgesic effect of Vtilb knockdown on thermal hypersensitivity is TRPV1-specific and not an "offtarget" effect, as the CFA-induced thermal hypersensitivity is already profoundly impaired in Trpv1-KO mice (Caterina et al., 2000). For this reason, measuring the expression and secretion of inflammatory mediators and their targets should reveal whether knockdown of Vtilb in sensory neurons rather dampens the overall inflammatory response to CFA injection which also would also lead to a diminished TRPV1 sensitisation.

As my investigation of the Vti1b knockdown on pain models in vivo, relied mainly on the assessment of changes in nociceptive thresholds, i.e. stimulus-evoked behaviour, the validity of my findings is limited in some respects. Clinical manifestations of chronic pain are not 
only accompanied by hypersensitivity to external stimuli, but also by ongoing, spontaneous pain along with the effects on the mood of the patient/patient's quality of life. However, parameters other than stimulus-evoked behaviour are difficult to assess in rodents and this represents a major limitation in the use of animals in pain research (Tappe-Theodor and Kuner, 2014). This is increasingly recognized by researchers and in recent years, several surrogate behavioural measurements, e.g. changes in voluntary behaviour and sociability, have been investigated for their applicability and validity for assessing animal well-being in rodent pain models (Cobos et al., 2012; Pitzer et al., 2016; Urban et al., 2011).

Including these non-evoked measures into future studies would help with defining the relevance of the Vti1b-TRPV1 interaction for nociception and pain in vivo.

The mass spectrometry-based screen for state-specific constituents of the TRPV1 protein complex proved the interaction with Vtilb and also detected that a part of the TRPV1 interactome undergoes significant changes under inflammatory pain conditions. This adds to the notion that dynamic and pain-specific PPIs exist. Blocking TRPV1 has been shown have an analgesic effect in many different chronic pain models. Yet, TRPV1-antagonists have been challenged by severe side effects in preclinical trials due to interference with the physiological functions of TRPV1. Therefore, defining the proteins modulating TRPV1 activity under pathological conditions is a crucial step for the development of novel drugs that target PPIs specific for pathological pain, whilst leaving nociceptive pain untouched. My list of candidates may therefore provide a valuable resource for further investigation on PPIs specific for inflammatory pain. Especially, novel proteins that have not yet been investigated in the peripheral nervous system/nociception are interesting targets to follow up. 


\section{Bibliography}

Aebersold, R., and Mann, M. (2003). Mass spectrometry-based proteomics. Nature 422, 198-207.

Ahluwalia, J., Rang, H., and Nagy, I. (2002). The putative role of vanilloid receptor-like protein-1 in mediating high threshold noxious heat-sensitivity in rat cultured primary sensory neurons. Eur. J. Neurosci. 16, 1483-1489.

Akopian, A.N., Ruparel, N.B., Jeske, N.A., and Hargreaves, K.M. (2007). Transient receptor potential TRPA 1 channel desensitization in sensory neurons is agonist dependent and regulated by TRPV1-directed internalization. J. Physiol. 583, 175-193.

Alberts, B. (1998). The Cell as a Collection Overview of Protein Machines: Preparing the Next Generation of Molecular Biologists. Cell 92, 291-294.

Alessandri-Haber, N., Dina, O.A., Chen, X., and Levine, J.D. (2009). TRPC1 and TRPC6 channels cooperate with TRPV4 to mediate mechanical hyperalgesia and nociceptor sensitization. J Neurosci 29, 6217-6228.

Alvarado, S., Tajerian, M., Millecamps, M., Suderman, M., Stone, L.S., and Szyf, M. (2013). Peripheral Nerve Injury is Accompanied by Chronic Transcriptome-Wide Changes in the Mouse Prefrontal Cortex. Mol. Pain 9, 1744-8069-9-21.

Amaya, F., Oh-Hashi, K., Naruse, Y., Iijima, N., Ueda, M., Shimosato, G., Tominaga, M., Tanaka, Y., and Tanaka, M. (2003). Local inflammation increases vanilloid receptor 1 expression within distinct subgroups of DRG neurons. Brain Res. 963, 190-196.

Amir, R., Liu, C.-N., Kocsis, J.D., and Devor, M. (2002). Oscillatory mechanism in primary sensory neurones. Brain 125, 421-435.

Antonin, W., Holroyd, C., Fasshauer, D., Pabst, S., Fischer von Mollard, G., and Jahn, R. (2000). A SNARE complex mediating fusion of late endosomes defines conserved properties of SNARE structure and function. Embo J 19, 6453-6464.

Antunes-Martins, A., Perkins, J.R., Lees, J., Hildebrandt, T., Orengo, C., and Bennett, D.L.H. (2013). Systems biology approaches to finding novel pain mediators. Wiley Interdiscip. Rev. Syst. Biol. Med. 5, 11-35.

Apkarian, A.V., Bushnell, M.C., Treede, R.D., and Zubieta, J.K. (2005). Human brain mechanisms of pain perception and regulation in health and disease. Eur. J. Pain 9, 463484.

Ashburner, M., Ball, C.A., Blake, J.A., Botstein, D., Butler, H., Cherry, J.M., Davis, A.P., Dolinski, K., Dwight, S.S., Eppig, J.T., et al. (2000). Gene Ontology: tool for the unification of biology. Nat. Genet. 25, 25-29.

Atlashkin, V., Kreykenbohm, V., Eskelinen, E.-L., Wenzel, D., Fayyazi, A., and Fischer von Mollard, G. (2003). Deletion of the SNARE vtilb in mice results in the loss of a single SNARE partner, syntaxin 8. Mol. Cell. Biol. 23, 5198-5207.

Avelino, A., and Cruz, F. (2006). TRPV1 (vanilloid receptor) in the urinary tract: Expression, function and clinical applications. Naunyn. Schmiedebergs. Arch. Pharmacol. $373,287-299$. 
Baloh, R.H. (2008). Mitochondrial Dynamics and Peripheral Neuropathy. Neurosci. 14, $12-18$.

Barabási, A., Gulbahce, N., and Loscalzo, J. (2011). Network medicine : a network-based approach to human disease. Nat. Publ. Gr. 12, 56-68.

Barry, A.M., Sondermann, J.R., Sondermann, J.-H., Gomez-Varela, D., and Schmidt, M. (2018). Region-resolved quantitative proteome profiling reveals molecular dynamics associated with chronic pain in the PNS and spinal cord. Front. Mol. Neurosci. 11, 259.

Basbaum, A.I., and Jessell, T.M. (2013). Part V: Perception, Chapter 24: Pain. In Principles of Neural Science, E.R. Kandel, and et al., eds. (New York : McGraw-Hill Medical), pp. 530-555.

Basbaum, A.I., Bautista, D.M., Scherrer, G., and Julius, D. (2009). Cellular and molecular mechanisms of pain. Cell 139, 267-284.

Bautista, D.M., Movahed, P., Hinman, A., Axelsson, H.E., Sterner, O., Hogestatt, E.D., Julius, D., Jordt, S.-E., and Zygmunt, P.M. (2005). Pungent products from garlic activate the sensory ion channel TRPA1. Proc. Natl. Acad. Sci. 102, 12248-12252.

Bautista, D.M., Jordt, S.E., Nikai, T., Tsuruda, P.R., Read, A.J., Poblete, J., Yamoah, E.N., Basbaum, A.I., and Julius, D. (2006). TRPA1 Mediates the Inflammatory Actions of Environmental Irritants and Proalgesic Agents. Cell 124, 1269-1282.

Bavassano, C., Marvaldi, L., Langeslag, M., Sarg, B., Lindner, H., Klimaschewski, L., Kress, M., Ferrer-Montiel, A., and Knaus, H.-G. (2013). Identification of voltage-gated $\mathrm{K}(+)$ channel beta $2(\mathrm{Kv} \beta 2)$ subunit as a novel interaction partner of the pain transducer Transient Receptor Potential Vanilloid 1 channel (TRPV1). Biochim. Biophys. Acta 1833, 3166-3175.

Baxter, R. V, Ben Othmane, K., Rochelle, J.M., Stajich, J.E., Hulette, C., Dew-Knight, S., Hentati, F., Ben Hamida, M., Bel, S., Stenger, J.E., et al. (2002). Ganglioside-induced differentiation-associated protein-1 is mutant in Charcot-Marie-Tooth disease type 4A/8q21. Nat. Genet. 30, 21-22.

Beecher, H.K. (1946). PAIN IN MEN WOUNDED IN BATTLE. Ann. Surg. 123, 96-105.

Bhave, G., Zhu, W., Wang, H., Brasier, D.J., Oxford, G.S., and Gereau IV, R.W. (2002). cAMP-dependent protein kinase regulates desensitization of the capsaicin receptor ( VR1) by direct phosphorylation. Neuron $35,721-731$.

Bhave, G., Hu, H.-J., Glauner, K.S., Zhu, W., Wang, H., Brasier, D.J., Oxford, G.S., Gereau, R.W., and IV (2003). Protein kinase C phosphorylation sensitizes but does not activate the capsaicin receptor transient receptor potential vanilloid 1 (TRPV1). Proc. Natl. Acad. Sci. U. S. A. 100, 12480-12485.

Bianchi, R., Cervellini, I., Porretta-Serapiglia, C., Oggioni, N., Burkey, B., Ghezzi, P., Cavaletti, G., and Lauria, G. (2011). Beneficial effects of PKF275-055, a novel, selective, and orally bioavailable, long-acting dipeptidyl peptidase IV inhibitor in streptozotocininduced diabetic peripheral neuropathy. J. Pharmacol. Exp. Ther. 340, 64-72.

Blazer, L.L., and Neubig, R.R. (2009). Small Molecule Protein-Protein Interaction Inhibitors as CNS Therapeutic Agents: Current Progress and Future Hurdles. Neuropsychopharmacology 34, 126-141. 
Bonnington, J.K., and McNaughton, P.A. (2003). Signalling pathways involved in the sensitisation of mouse nociceptive neurones by nerve growth factor. J. Physiol. 551, 433446.

Borsook, D., Hargreaves, R., Bountra, C., and Porreca, F. (2014). Lost but making progress - Where will new analgesic drugs come from ? Sci. Transl. Med. 6, 1-11.

Bourquin, A.-F., Süveges, M., Pertin, M., Gilliard, N., Sardy, S., Davison, A.C., Spahn, D.R., and Decosterd, I. (2006). Assessment and analysis of mechanical allodynia-like behavior induced by spared nerve injury (SNI) in the mouse. Pain 122, 14.e1-14.

Breivik, H., Collett, B., Ventafridda, V., Cohen, R., and Gallacher, D. (2006). Survey of chronic pain in Europe: Prevalence, impact on daily life, and treatment. Eur. J. Pain 10, 287-333.

Breivik, H., Eisenberg, E., and O'Brien, T. (2013). The individual and societal burden of chronic pain in Europe: the case for strategic prioritisation and action to improve knowledge and availability of appropriate care. BMC Public Health 13, 1229.

Bruderer, R., Bernhardt, O.M., Gandhi, T., Miladinović, S.M., Cheng, L.-Y., Messner, S., Ehrenberger, T., Zanotelli, V., Butscheid, Y., Escher, C., et al. (2015). Extending the Limits of Quantitative Proteome Profiling with Data-Independent Acquisition and Application to Acetaminophen-Treated Three-Dimensional Liver Microtissues. Mol. Cell. Proteomics 14, 1400-1410.

Btesh, J., Fischer, M.J.M., Stott, K., and McNaughton, P. a (2013). Mapping the binding site of TRPV1 on AKAP79: implications for inflammatory hyperalgesia. J. Neurosci. 33, 9184-9193.

Butler, R.K., and Finn, D.P. (2009). Stress-induced analgesia. Prog. Neurobiol. 88, 184202.

Camprubí-Robles, M., Planells-Cases, R., and Ferrer-Montiel, A. (2009). Differential contribution of SNARE-dependent exocytosis to inflammatory potentiation of TRPV1 in nociceptors. FASEB J. 23, 3722-3733.

Cao, E., Liao, M., Cheng, Y., and Julius, D. (2013). TRPV1 structures in distinct conformations reveal activation mechanisms. Nature 504, 113-118.

Carlton, S.M., and Coggeshall, R.E. (2001). Peripheral capsaicin receptors increase in the inflamed rat hindpaw: A possible mechanism for peripheral sensitization. Neurosci. Lett. $310,53-56$.

Carnevale, V., and Rohacs, T. (2016). TRPV1: A target for rational drug design. Pharmaceuticals 9, 1-20.

Carpenter, S.E., and Lynn, B. (1981). Vascular and Sensory Responses of Human Skin To Mild Injury After Topical Treatment With Capsaicin. Br. J. Pharmacol. 73, 755-758.

Caterina, M.J., Schumacher, M.A., Tominaga, M., Rosen, T.A., Levine, J.D., and Julius, D. (1997). The capsaicin receptor: a heat-activated ion channel in the pain pathway. Nature $389,816-824$.

Caterina, M.J., Rosen, T. a, Tominaga, M., Brake, a J., and Julius, D. (1999). A capsaicinreceptor homologue with a high threshold for noxious heat. Nature 398, 436-441.

Caterina, M.J., Leffler, A., Malmberg, A.B., Martin, W.J., Trafton, J., Petersen-Zeitz, K.R., 
Koltzenburg, M., Basbaum, A.I., and Julius, D. (2000). Impaired nociception and pain sensation in mice lacking the capsaicin receptor. Science 288, 306-313.

Cavanaugh, D.J., Lee, H., Lo, L., Shields, S.D., Zylka, M.J., Basbaum, A.I., and Anderson, D.J. (2009). Distinct subsets of unmyelinated primary sensory fibers mediate behavioral responses to noxious thermal and mechanical stimuli. Proc. Natl. Acad. Sci. 106, 90759080.

Cavanaugh, D.J., Chesler, A.T., Jackson, A.C., Sigal, Y.M., Yamanaka, H., Grant, R., Donnell, D.O., Nicoll, R.A., Shah, N.M., Julius, D., et al. (2011). Trpv1 reporter mice reveal highly restricted brain distribution and functional expression in arteriolar smooth muscle cells. J. Neurosci. 31, 5067-5077.

Chakrabarty, A., Liao, Z., and Smith, P.G. (2013). Angiotensin II receptor type 2 activation is required for cutaneous sensory hyperinnervation and hypersensitivity in a rat hind paw model of inflammatory Pain. J. Pain 14, 1053-1065.

Chen, Y.A., and Scheller, R.H. (2001). SNARE-mediated membrane fusion. Nat. Rev. Mol. Cell Biol. 2, 98-106.

Chen, B., Zhao, L., Li, X., Ji, Y.S., Li, N., Xu, X.F., and Chen, Z.Y. (2014). Syntaxin 8 modulates the post-synthetic trafficking of the TrkA receptor and inflammatory pain transmission. J. Biol. Chem. 289, 19556-19569.

Choudhary, C., and Mann, M. (2010). Decoding signalling networks by mass spectrometry-based proteomics. Nat. Rev. Mol. Cell Biol. 11, 427-439.

Christoph, T., Grünweller, A., Mika, J., Schäfer, M.K.H., Wade, E.J., Weihe, E., Erdmann, V.A., Frank, R., Gillen, C., and Kurreck, J. (2006). Silencing of vanilloid receptor TRPV1 by RNAi reduces neuropathic and visceral pain in vivo. Biochem. Biophys. Res. Commun. $350,238-243$.

Christoph, T., Bahrenberg, G., De Vry, J., Englberger, W., Erdmann, V.A., Frech, M., Kögel, B., Röhl, T., Schiene, K., Schröder, W., et al. (2008). Investigation of TRPV1 lossof-function phenotypes in transgenic shRNA expressing and knockout mice. Mol. Cell. Neurosci. 37, 579-589.

Chuang, H.H., Prescott, E.D., Kong, H., Shields, S., Jordt, S.E., Basbaum, A.I., Chao, M. V, and Julius, D. (2001). Bradykinin and nerve growth factor release the capsaicin receptor from PtdIns(4,5)P2-mediated inhibition. Nature 411, 957-962.

Clapham, D.E. (2003). TRP channels as cellular sensors. Nature 426, 517-524.

Cobos, E.J., Ghasemlou, N., Araldi, D., Segal, D., Duong, K., and Woolf, C.J. (2012). Inflammation-induced decrease in voluntary wheel running in mice: a non-reflexive test for evaluating inflammatory pain and analgesia. Pain 153, 876-884.

Collins, B.C., Hunter, C.L., Liu, Y., Schilling, B., Rosenberger, G., Bader, S.L., Chan, D.W., Gibson, B.W., Gingras, A.-C., Held, J.M., et al. (2017). Multi-laboratory assessment of reproducibility, qualitative and quantitative performance of SWATH-mass spectrometry. Nat. Commun. 8, 291.

da Costa, D.S.M., Meotti, F.C., Andrade, E.L., Leal, P.C., Motta, E.M., and Calixto, J.B. (2010). The involvement of the transient receptor potential A1 (TRPA1) in the maintenance of mechanical and cold hyperalgesia in persistent inflammation. Pain 148, 431-437. 
Costigan, M., Scholz, J., and Woolf, C.J. (2009). Neuropathic pain: a maladaptive response of the nervous system to damage. Annu. Rev. Neurosci. 32, 1-32.

Cox, J.J., Reimann, F., Nicholas, A.K., Thornton, G., Roberts, E., Springell, K., Karbani, G., Jafri, H., Mannan, J., Raashid, Y., et al. (2006). An SCN9A channelopathy causes congenital inability to experience pain. Nature 444, 894-898.

Cristino, L., de Petrocellis, L., Pryce, G., Baker, D., Guglielmotti, V., and Di Marzo, V. (2006). Immunohistochemical localization of cannabinoid type 1 and vanilloid transient receptor potential vanilloid type 1 receptors in the mouse brain. Neuroscience 139, 14051415.

Davis, J.B., Gray, J., Gunthorpe, M.J., Hatcher, J.P., Davey, P.T., Overend, P., Harries, M.H., Latcham, J., Clapham, C., Atkinson, K., et al. (2000). Vanilloid receptor-1 is essential for inflammatory thermal hyperalgesia. Nature 405, 183-187.

Decosterd, I., and Woolf, C.J. (2000). Spared nerve injury: an animal model of persistent peripheral neuropathic pain. Pain 87, 149-158.

Delmas, P. (2008). SnapShot: Ion Channels and Pain. Cell 134, 366.e1.

Deutsch, E.W., Lam, H., and Aebersold, R. (2008). PeptideAtlas: a resource for target selection for emerging targeted proteomics workflows. EMBO Rep. 9, 429-434.

Distler, U., Kuharev, J., Navarro, P., Levin, Y., Schild, H., and Tenzer, S. (2014). Drift time-specific collision energies enable deep-coverage data-independent acquisition proteomics. Nat. Methods 11, 167-170.

Distler, U., Kuharev, J., Navarro, P., and Tenzer, S. (2016). Label-free quantification in ion mobility-enhanced data-independent acquisition proteomics. Nat. Protoc. 11, 795-812.

Docherty, R.J., Yeats, J.C., Bevan, S., and Boddeke, H.W.G.M. (1996). Inhibition of calcineurin inhibits the desensitization of capsaicin-evoked currents in cultured dorsal root ganglion neurones from adult rats. Pflugers Arch. Eur. J. Physiol. Arch. Eur. J. Physiol. $431,828-837$.

Domon, B., and Aebersold, R. (2010). Options and considerations when selecting a quantitative proteomics strategy. Nat. Biotechnol. 28, 710-721.

Dressel, R., Elsner, L., Novota, P., Kanwar, N., and Fischer von Mollard, G. (2010). The exocytosis of lytic granules is impaired in Vti1b- or Vamp8-deficient CTL leading to a reduced cytotoxic activity following antigen-specific activation. J. Immunol. 185, 10051014.

Dubin, A.E., and Patapoutian, A. (2010). Nociceptors : the sensors of the pain pathway. J. Clin. Invest. 120, 3760-3772.

Dueñas, M., Ojeda, B., Salazar, A., Mico, J.A., and Failde, I. (2016). A review of chronic pain impact on patients, their social environment and the health care system. J. Pain Res. 9, 457-467.

Fehrenbacher, J.C., Vasko, M.R., and Duarte, D.B. (2012). Models of Inflammation: Carrageenan- or Complete Freund's Adjuvant-Induced Edema and Hypersensitivity in the Rat. Curr Protoc Pharmacol 56, 5.4.1-5.4.7.

Fernández-Carvajal, A., Fernandez-Ballester, G., Devesa, I., González-Ros, J.M., and Ferrer-Montiel, A. (2012). New strategies to develop novel pain therapies: Addressing 
thermoreceptors from different points of view. Pharmaceuticals 5, 16-48.

Fernyhough, P., Roy Chowdhury, S.K., and Schmidt, R.E. (2010). Mitochondrial stress and the pathogenesis of diabetic neuropathy. Expert Rev. Endocrinol. Metab. 5, 39-49.

Ferrandiz-Huertas, C., Mathivanan, S., Wolf, C.J., Devesa, I., and Ferrer-Montiel, A. (2014). Trafficking of ThermoTRP Channels. Membranes (Basel). 4, 525-564.

Ferrari, L.F., Chum, A., Bogen, O., Reichling, D.B., and Levine, J.D. (2011). Role of Drp1, a key mitochondrial fission protein, in neuropathic pain. J. Neurosci. 31, 1140411410.

Fischer, M.J.M., Btesh, J., and McNaughton, P.A. (2013). Disrupting sensitization of transient receptor potential vanilloid subtype 1 inhibits inflammatory hyperalgesia. J. Neurosci. 33, 7407-7414.

Fukuoka, T., Tokunaga, A., Tachibana, T., Dai, Y., Yamanaka, H., and Noguchi, K. (2002). VR1, but not P2X3, increases in the spared L4 DRG in rats with L5 spinal nerve ligation. Pain 99, 111-120.

Fusco, M. De, Marconi, R., Silvestri, L., Atorino, L., Rampoldi, L., Morgante, L., Ballabio, A., Aridon, P., and Casari, G. (2003). Haploinsufficiency of ATP1A2 encoding the $\mathrm{Na}+/ \mathrm{K}+$ pump $\alpha 2$ subunit associated with familial hemiplegic migraine type 2 . Nat. Genet. 33, 192-196.

García-Ferreiro, R.E., Kerschensteiner, D., Major, F., Monje, F., Stühmer, W., and Pardo, L.A. (2004). Mechanism of Block of hEag1 $\mathrm{K}^{+}$Channels by Imipramine and Astemizole. J. Gen. Physiol. 124, 301-317.

Gavva, N.R., Tamir, R., Qu, Y., Klionsky, L., Zhang, T.J., Immke, D., Wang, J., Zhu, D., Vanderah, T.W., Porreca, F., et al. (2005). AMG 9810 [(E)-3-(4-t-Butylphenyl)-N-(2,3dihydrobenzo[b][1,4] dioxin-6-yl)acrylamide], a Novel Vanilloid Receptor 1 (TRPV1) Antagonist with Antihyperalgesic Properties. J. Pharmacol. Exp. Ther. 313, 474-484.

Gavva, N.R., Bannon, A.W., Surapaneni, S., Hovland, D.N., Lehto, S.G., Gore, A., Juan, T., Deng, H., Han, B., Klionsky, L., et al. (2007). The Vanilloid Receptor TRPV1 Is Tonically Activated In Vivo and Involved in Body Temperature Regulation. J. Neurosci. $27,3366-3374$.

Gavva, N.R., Treanor, J.J.S., Garami, A., Fang, L., Surapaneni, S., Akrami, A., Alvarez, F., Bak, A., Darling, M., Gore, A., et al. (2008). Pharmacological blockade of the vanilloid receptor TRPV1 elicits marked hyperthermia in humans. Pain 136, 202-210.

Gerold, G., Meissner, F., Bruening, J., Welsch, K., Perin, P.M., Baumert, T.F., Vondran, F.W., Kaderali, L., Marcotrigiano, J., Khan, A.G., et al. (2015). Quantitative Proteomics Identifies Serum Response Factor Binding Protein 1 as a Host Factor for Hepatitis C Virus Entry. Cell Rep. 12, 864-878.

Gibson, H.E., Edwards, J.G., Page, R.S., Van Hook, M.J., and Kauer, J.A. (2008). TRPV1 Channels Mediate Long-Term Depression at Synapses on Hippocampal Interneurons. Neuron 57, 746-759.

Gibson, T.J., Seiler, M., and Veitia, R.A. (2013). The transience of transient overexpression. Nat. Methods 10, 715-721.

Gillet, L.C., Navarro, P., Tate, S., Rost, H., Selevsek, N., Reiter, L., Bonner, R., and 
Aebersold, R. (2012). Targeted Data Extraction of the MS/MS Spectra Generated by Dataindependent Acquisition: A New Concept for Consistent and Accurate Proteome Analysis. Mol. Cell. Proteomics 11, O111.016717-O111.016717.

Glatzel, M., Flechsig, E., Navarro, B., Klein, M.A., Paterna, J.C., Büeler, H., and Aguzzi, A. (2000). Adenoviral and adeno-associated viral transfer of genes to the peripheral nervous system. Proc. Natl. Acad. Sci. U. S. A. 97, 442-447.

Gold, M.S., and Gebhart, G.F. (2010). Nociceptor sensitization in pain pathogenesis. Nat. Med. 16, 1248-1257.

Gomez-Varela, D., and Schmidt, M. (2016). Exploring novel paths towards protein signatures of chronic pain. Mol. Pain 12, 1-5.

Gregory, N., Harris, A., Robinson, C., Dougherty, P., Fuchs, P., and Sluka, K. (2013). An overview of animal models of pain: disease models and outcome measures. J Pain 14, 1255-1269.

Guieu, R., Fenouillet, E., Devaux, C., Fajloun, Z., Carrega, L., Sabatier, J.M., Sauze, N., and Marguet, D. (2006). CD26 modulates nociception in mice via its dipeptidyl-peptidase IV activity. Behav. Brain Res. 166, 230-235.

Hanack, C., Moroni, M., Lima, W.C., Wende, H., Kirchner, M., Adelfinger, L., SchrenkSiemens, K., Tappe-Theodor, A., Wetzel, C., Kuich, P.H., et al. (2015). GABA blocks pathological but not acute TRPV1 pain signals. Cell 160, 759-770.

Hargreaves, K., Dubner, R., Brown, F., Flores, C., and Joris, J. (1988). A new and sensitive method for measuring thermal nociception in cutaneous hyperalgesia. Pain 32, 77-88.

He, M.-L., Zemkova, H., Koshimizu, T., Tomić, M., and Stojilkovic, S.S. (2003). Intracellular calcium measurements as a method in studies on activity of purinergic $\mathrm{P} 2 \mathrm{X}$ receptor channels. Am. J. Physiol. Cell Physiol. 285, C467-79.

von Hehn, C.A., Baron, R., and Woolf, C.J. (2012). Deconstructing the Neuropathic Pain Phenotype to Reveal Neural Mechanisms. Neuron 73, 638-652.

Helbig, A.O., Heck, A.J.R., and Slijper, M. (2010). Exploring the membrane proteome-challenges and analytical strategies. J. Proteomics 73, 868-878.

Helliwell, R.J., McLatchie, L.M., Clarke, M., Winter, J., Bevan, S., and McIntyre, P. (1998). Capsaicin sensitivity is associated with the expression of the vanilloid (capsaicin) receptor (VR1) mRNA in adult rat sensory ganglia. Neurosci. Lett. 250, 177-180.

Hellwig, N., Albrecht, N., Harteneck, C., Schultz, G., and Schaefer, M. (2005). Homo- and heteromeric assembly of TRPV channel subunits. J. Cell Sci. 118, 917-928.

Holland, S., Coste, O., Zhang, D.D., Pierre, S.C., Geisslinger, G., and Scholich, K. (2011). The ubiquitin ligase MYCBP2 regulates transient receptor potential vanilloid receptor 1 (TRPV1) internalization through inhibition of p38 MAPK signaling. J. Biol. Chem. 286, 3671-3680.

Huang, D.W., Sherman, B.T., and Lempicki, R.A. (2008a). Systematic and integrative analysis of large gene lists using DAVID bioinformatics resources. Nat. Protoc. 4, 44-57.

Huang, H.-L., Cendan, C.-M., Roza, C., Okuse, K., Cramer, R., Timms, J.F., and Wood, J.N. (2008b). Proteomic profiling of neuromas reveals alterations in protein composition and local protein synthesis in hyper-excitable nerves. Mol. Pain 4, 33. 
Hubner, N.C., and Mann, M. (2011). Extracting gene function from protein-protein interactions using Quantitative BAC InteraCtomics (QUBIC). Methods 53, 453-459.

Hubner, N.C., Bird, A.W., Cox, J., Splettstoesser, B., Bandilla, P., Poser, I., Hyman, A., and Mann, M. (2010). Quantitative proteomics combined with BAC TransgeneOmics reveals in vivo protein interactions. J. Cell Biol. 189, 739-754.

Hudson, L.J., Bevan, S., Wotherspoon, G., Gentry, C., Fox, A., and Winter, J. (2001). VR1 protein expression increases in undamaged DRG neurons after partial nerve injury. Eur. J. Neurosci. 13, 2105-2114.

Inceoglu, B., Bettaieb, A., Trindade da Silva, C.A., Lee, K.S.S., Haj, F.G., and Hammock, B.D. (2015). Endoplasmic reticulum stress in the peripheral nervous system is a significant driver of neuropathic pain. Proc. Natl. Acad. Sci. 112, 9082-9087.

Indo, Y., Tsuruta, M., Hayashida, Y., Karim, M.A., Ohta, K., Kawano, T., Mitsubuchi, H., Tonoki, H., Awaya, Y., and Matsuda, I. (1996). Mutations in the TRKA/NGF receptor gene in patients with congenital insensitivity to pain with anhidrosis. Nat. Genet. 13, 485488.

Inoue, K., Koizumi, S., Fuziwara, S., Denda, S., Inoue, K., and Denda, M. (2002). Functional Vanilloid Receptors in Cultured Normal Human Epidermal Keratinocytes. Biochem. Biophys. Res. Commun. 291, 124-129.

Jeske, N.A., Diogenes, A., Ruparel, N.B., Fehrenbacher, J.C., Henry, M., Akopian, A.N., and Hargreaves, K.M. (2008). A-kinase anchoring protein mediates TRPV1 thermal hyperalgesia through PKA phosphorylation of TRPV1. Pain 138, 604-616.

Jeske, N.A., Patwardhan, A.M., Ruparel, N.B., Akopian, A.N., Shapiro, M.S., and Henry, M.A. (2009). A-kinase anchoring protein 150 controls protein kinase C-mediated phosphorylation and sensitization of TRPV1. Pain 146, 301-307.

Ji, R.R., Samad, T.A., Jin, S.X., Schmoll, R., and Woolf, C.J. (2002). p38 MAPK activation by NGF in primary sensory neurons after inflammation increases TRPV1 levels and maintains heat hyperalgesia. Neuron 36, 57-68.

Jordt, S.-E., Tominaga, M., and Julius, D. (2000). Acid potentiation of the capsaicin receptor determined by a key extracellular site. Proc. Natl. Acad. Sci. 97, 8134-8139.

Joseph, E.K., and Levine, J.D. (2006). Mitochondrial electron transport in models of neuropathic and inflammatory pain. Pain 121, 105-114.

Joseph, E.K., and Levine, J.D. (2009). Comparison of Oxaliplatin- and Cisplatin-Induced Painful Peripheral Neuropathy in the Rat. J. Pain 10, 534-541.

Julius, D. (2013). TRP channels and pain. Annu Rev Cell Dev Biol 29, 355-384.

Kanai, Y., Nakazato, E., Fujiuchi, A., Hara, T., and Imai, A. (2005). Involvement of an increased spinal TRPV1 sensitization through its up-regulation in mechanical allodynia of CCI rats. Neuropharmacology 49, 977-984.

Kanai, Y., Hara, T., Imai, A., and Sakakibara, A. (2007). Differential involvement of TRPV1 receptors at the central and peripheral nerves in CFA-induced mechanical and thermal hyperalgesia. J. Pharm. Pharmacol. 59, 733-738.

Kaneko, Y., and Szallasi, A. (2014). Transient receptor potential (TRP) channels: A clinical perspective. Br. J. Pharmacol. 171, 2474-2507. 
Kauer, J.A., and Gibson, H.E. (2009). Hot flash: TRPV channels in the brain. Trends Neurosci. 32, 215-224.

Keilhauer, E.C., Hein, M.Y., and Mann, M. (2015). Accurate protein complex retrieval by affinity enrichment mass spectrometry (AE-MS) rather than affinity purification mass spectrometry (AP-MS). Mol. Cell. Proteomics 14, 120-135.

Kim, H.K., Park, S.K., Zhou, J.-L., Taglialatela, G., Chung, K., Coggeshall, R.E., and Chung, J.M. (2004). Reactive oxygen species (ROS) play an important role in a rat model of neuropathic pain. Pain 111, 116-124.

Kim, H.Y., Park, C.K., Cho, I.H., Jung, S.J., Kim, J.S., and Oh, S.B. (2008). Differential changes in TRPV1 expression after trigeminal sensory nerve injury. J. Pain 9, 280-288.

Kollarik, M., and Undem, B.J. (2004). Activation of bronchopulmonary vagal afferent nerves with bradykinin, acid and vanilloid receptor agonists in wild-type and TRPV1-/mice. J. Physiol. 555, 115-123.

Kollarik, M., Ru, F., and Brozmanova, M. (2010). Vagal afferent nerves with the properties of nociceptors. Auton. Neurosci. Basic Clin. 153, 12-20.

Koplas P. A., Rosenberg R. L., and Oxford G. S. (1997). The role of calcium in the desensitization of capsaicin responses in rat dorsal root ganglion neurons. J. Neurosci. 17, $3525-3537$.

Kremeyer, B., Lopera, F., Cox, J.J., Momin, A., Rugiero, F., Marsh, S., Woods, C.G., Jones, N.G., Paterson, K.J., Fricker, F.R., et al. (2010). A Gain-of-Function Mutation in TRPA1 Causes Familial Episodic Pain Syndrome. Neuron 66, 671-680.

Kreykenbohm, V., Wenzel, D., Antonin, W., Atlachkine, V., and von Mollard, G.F. (2002). The SNAREs vtila and vtilb have distinct localization and SNARE complex partners. Eur. J. Cell Biol. 81, 273-280.

Kroenke, K., Krebs, E.E., and Bair, M.J. (2009). Pharmacotherapy of chronic pain: a synthesis of recommendations from systematic reviews. Gen. Hosp. Psychiatry 31, 206219.

Kuharev, J., Navarro, P., Distler, U., Jahn, O., and Tenzer, S. (2015). In-depth evaluation of software tools for data-independent acquisition based label-free quantification.

Proteomics 15, 3140-3151.

Kuner, R. (2010). Central mechanisms of pathological pain. Nat Med 16, 1258-1266.

Kuner, R., and Flor, H. (2016). Structural plasticity and reorganisation in chronic pain.

Nat. Rev. Neurosci. 18, 20-30.

Kunwar, A.J., Rickmann, M., Backofen, B., Browski, S.M., Rosenbusch, J., Schöning, S., Fleischmann, T., Krieglstein, K., and Fischer von Mollard, G. (2011). Lack of the endosomal SNAREs vtila and vtilb led to significant impairments in neuronal development. Proc. Natl. Acad. Sci. U. S. A. 108, 2575-2580.

Kwan, K.Y., Allchorne, A.J., Vollrath, M.A., Christensen, A.P., Zhang, D.S., Woolf, C.J., and Corey, D.P. (2006). TRPA1 Contributes to Cold, Mechanical, and Chemical Nociception but Is Not Essential for Hair-Cell Transduction. Neuron 50, $277-289$.

de la Roche, J., Walther, I., Leonow, W., Hage, A., Eberhardt, M., Fischer, M., Reeh, P.W., Sauer, S., and Leffler, A. (2016). Lactate is a potent inhibitor of the capsaicin 
receptor TRPV1. Sci. Rep. 6, 36740.

Lacroix-Fralish, M.L., Austin, J.S., Zheng, F.Y., Levitin, D.J., and Mogil, J.S. (2011). Patterns of pain: Meta-analysis of microarray studies of pain. Pain 152, 1888-1898.

Laedermann, C.J., Pertin, M., Suter, M.R., and Decosterd, I. (2014). Voltage-gated sodium channel expression in mouse DRG after SNI leads to re-evaluation of projections of injured fibers. Mol. Pain 10, 19.

Laínez, S., Valente, P., Ontoria-Oviedo, I., Estévez-Herrera, J., Camprubí-Robles, M., Ferrer-Montiel, A., and Planells-Cases, R. (2010). GABAA receptor associated protein (GABARAP) modulates TRPV1 expression and channel function and desensitization. FASEB J. 24, 1958-1970.

Lambert, J.-P., Ivosev, G., Couzens, A.L., Larsen, B., Taipale, M., Lin, Z.-Y., Zhong, Q., Lindquist, S., Vidal, M., Aebersold, R., et al. (2013). Mapping differential interactomes by affinity purification coupled with data-independent mass spectrometry acquisition. Nat. Methods 10, 1239-1245.

Law, K.P., and Lim, Y.P. (2013). Recent advances in mass spectrometry: data independent analysis and hyper reaction monitoring. Expert Rev. Proteomics 10, 551-566.

Leffler, A., Fischer, M.J., Rehner, D., Kienel, S., Kistner, K., Sauer, S.K., Gavva, N.R., Reeh, P.W., and Nau, C. (2008). The vanilloid receptor TRPV1 is activated and sensitized by local anesthetics in rodent sensory neurons. J. Clin. Invest. 118, 763-776.

Leipold, E., Liebmann, L., Korenke, G.C., Heinrich, T., Gießelmann, S., Baets, J., Ebbinghaus, M., Goral, R.O., Stödberg, T., Hennings, J.C., et al. (2013). A de novo gainof-function mutation in SCN11A causes loss of pain perception. Nat. Genet. 45, 1399 1404.

Leuchowius, K.J., Weibrecht, I., and Söderberg, O. (2011). In situ proximity ligation assay for microscopy and flow cytometry. Curr. Protoc. Cytom. 56, 9.36.1-9.36.15.

Liao, M., Cao, E., Julius, D., and Cheng, Y. (2013). Structure of the TRPV1 ion channel determined by electron cryo-microscopy. Nature 504, 107-112.

Lin, Y.-T., Ro, L.-S., Wang, H.-L., and Chen, J.-C. (2011). Up-regulation of dorsal root ganglia BDNF and trkB receptor in inflammatory pain: an in vivo and in vitro study. J. Neuroinflammation $8,126$.

Lishko, P. V., Procko, E., Jin, X., Phelps, C.B., and Gaudet, R. (2007). The Ankyrin Repeats of TRPV1 Bind Multiple Ligands and Modulate Channel Sensitivity. Neuron 54, 905-918.

Liu, B. (2005). Functional Recovery from Desensitization of Vanilloid Receptor TRPV1 Requires Resynthesis of Phosphatidylinositol 4,5-Bisphosphate. J. Neurosci. 25, 48354843.

Liu, X.J., Gingrich, J.R., Vargas-Caballero, M., Dong, Y.N., Sengar, A., Beggs, S., Wang, S.-H., Ding, H.K., Frankland, P.W., and Salter, M.W. (2008). Treatment of inflammatory and neuropathic pain by uncoupling Src from the NMDA receptor complex. Nat. Med. 14, $1325-1332$.

Liu, Y., Chen, J., Sethi, A., Li, Q.K., Chen, L., Collins, B., Gillet, L.C.J., Wollscheid, B., Zhang, H., and Aebersold, R. (2014). Glycoproteomic Analysis of Prostate Cancer Tissues 
by SWATH Mass Spectrometry Discovers N-acylethanolamine Acid Amidase and Protein Tyrosine Kinase 7 as Signatures for Tumor Aggressiveness. Mol. Cell. Proteomics 13, 1753-1768.

Livak, K.J., and Schmittgen, T.D. (2001). Analysis of Relative Gene Expression Data Using Real- Time Quantitative PCR and the -2 Delta CT Method. Methods 25, 402-408.

Lu, A., Wisniewski, J.R., and Mann, M. (2009). Comparative Proteomic Profiling of Membrane Proteins in Rat Cerebellum, Spinal Cord, and Sciatic Nerve. J. Proteome Res. $8,2418-2425$.

Lukacs, V., Yudin, Y., Hammond, G.R., Sharma, E., Fukami, K., and Rohács, T. (2013). Distinctive changes in plasma membrane phosphoinositides underlie differential regulation of TRPV1 in nociceptive neurons. J. Neurosci. 33, 11451-11463.

Lupachyk, S., Watcho, P., Stavniichuk, R., Shevalye, H., and Obrosova, I.G. (2013). Endoplasmic reticulum stress plays a key role in the pathogenesis of diabetic peripheral neuropathy. Diabetes 62, 944-952.

Lynch, M.E., and Watson, C.P.N. (2006). The pharmacotherapy of chronic pain: a review. Pain Res. Manag. 11, 11-38.

Macia, E., Ehrlich, M., Massol, R., Boucrot, E., Brunner, C., and Kirchhausen, T. (2006). Dynasore, a cell-permeable inhibitor of dynamin. Dev. Cell 10, 839-850.

Mandadi, S., Numazaki, M., Tominaga, M., Bhat, M.B., Armati, P.J., and Roufogalis, B.D. (2004). Activation of protein kinase $C$ reverses capsaicin-induced calcium-dependent desensitization of TRPV1 ion channels. Cell Calcium 35, 471-478.

Mandadi, S., Tominaga, T., Numazaki, M., Murayama, N., Saito, N., Armati, P.J., Roufogalis, B.D., and Tominaga, M. (2006). Increased sensitivity of desensitized TRPV1 by PMA occurs through PKC $\varepsilon$-mediated phosphorylation at S800. Pain 123, 106-116.

Manteniotis, S., Lehmann, R., Flegel, C., Vogel, F., Hofreuter, A., Schreiner, B.S.P., Altmüller, J., Becker, C., Schöbel, N., Hatt, H., et al. (2013). Comprehensive RNA-Seq expression analysis of sensory ganglia with a focus on ion channels and GPCRs in trigeminal ganglia. PLoS One 8, 1-30.

Marsch, R., Foeller, E., Rammes, G., Bunck, M., Kössl, M., Holsboer, F., Zieglgänsberger, W., Landgraf, R., Lutz, B., and Wotjak, C.T. (2007). Reduced anxiety, conditioned fear, and hippocampal long-term potentiation in transient receptor potential vanilloid type 1 receptor-deficient mice. J. Neurosci. 27, 832-839.

Mathivanan, S., Devesa, I., Changeux, J.P., and Ferrer-Montiel, A. (2016). Bradykinin induces TRPV1 exocytotic recruitment in peptidergic nociceptors. Front. Pharmacol. 7, 112.

Matta, J.A., and Ahern, G.P. (2007). Voltage is a partial activator of rat thermosensitive TRP channels. J. Physiol. 585, 469-482.

McGaraughty, S., Chu, K.L., Brown, B.S., Zhu, C.Z., Zhong, C., Joshi, S.K., Honore, P., Faltynek, C.R., and Jarvis, M.F. (2008). Contributions of central and peripheral TRPV1 receptors to mechanically evoked and spontaneous firing of spinal neurons in inflamed rats. J. Neurophysiol. 100, 3158-3166.

McNamara, C.R., Mandel-Brehm, J., Bautista, D.M., Siemens, J., Deranian, K.L., Zhao, 
M., Hayward, N.J., Chong, J.A., Julius, D., Moran, M.M., et al. (2007). TRPA1 mediates formalin-induced pain. Proc. Natl. Acad. Sci. 104, 13525-13530.

Melemedjian, O.K., Yassine, H.N., Shy, A., and Price, T.J. (2013). Proteomic and functional annotation analysis of injured peripheral nerves reveals ApoE as a protein upregulated by injury that is modulated by metformin treatment. Mol. Pain 9, 14.

Meng, J., Wang, J., Steinhoff, M., and Dolly, J.O. (2016). TNF $\alpha$ induces co-trafficking of TRPV1/TRPA1 in VAMP1-containing vesicles to the plasmalemma via Munc181/syntaxin1/SNAP-25 mediated fusion. Sci. Rep. 6, 21226.

Merskey, H., and Bogduk, N. (1994). Classification of chronic pain: Descriptions of chronic pain syndromes and definitions of pain terms. IASP Press. Seattle 3, 210.

Michalski, A., Cox, J., and Mann, M. (2011). More than 100,000 detectable peptide species elute in single shotgun proteomics runs but the majority is inaccessible to datadependent LC-MS/MS. J. Proteome Res. 10, 1785-1793.

Mickle, A.D., Shepherd, A.J., and Mohapatra, D.P. (2015). Sensory TRP channels: The key transducers of nociception and pain. Prog. Mol. Biol. Transl. Sci. 131, 73-118.

Mickle, A.D., Shepherd, A.J., and Mohapatra, D.P. (2016). Nociceptive TRP channels: Sensory detectors and transducers in multiple pain pathologies. Pharmaceuticals 9, 1-26.

Millan, M.J. (2002). Descending control of pain. Prog. Neurobiol. 66, 355-474.

Minett, M.S., Quick, K., and Wood, J.N. (2013). Behavioral measures of pain thresholds. Curr. Protoc. Mouse Biol. 1, 383-412.

Minke, B. (2010). The History of the Drosophila TRP Channel: The Birth of a New Channel Superfamily. J. Neurogenet. 24, 216-233.

Mohapatra, D.P., and Nau, C. (2003). Desensitization of Capsaicin-activated Currents in the Vanilloid Receptor TRPV1 Is Decreased by the Cyclic AMP-dependent Protein Kinase Pathway. J. Biol. Chem. 278, 50080-50090.

Mohapatra, D.P., and Nau, C. (2005). Regulation of Ca2+-dependent desensitization in the vanilloid receptor TRPV1 by calcineurin and cAMP-dependent protein kinase. J. Biol.

Chem. 280, 13424-13432.

Morenilla-Palao, C., Planells-Cases, R., García-Sanz, N., and Ferrer-Montiel, A. (2004). Regulated exocytosis contributes to protein kinase $\mathrm{C}$ potentiation of vanilloid receptor activity. J. Biol. Chem. 279, 25665-25672.

Murray, R.Z., Wylie, F.G., Khromykh, T., Hume, D.A., and Stow, J.L. (2005). Syntaxin 6 and Vtilb form a novel SNARE complex, which is up-regulated in activated macrophages to facilitate exocytosis of tumor necrosis factor alpha. J. Biol. Chem. 280, 10478-10483.

Musella, A., De Chiara, V., Rossi, S., Prosperetti, C., Bernardi, G., Maccarrone, M., and Centonze, D. (2009). TRPV1 channels facilitate glutamate transmission in the striatum. Mol. Cell. Neurosci. 40, 89-97.

Nadal, M.S., Ozaita, A., Amarillo, Y., Vega-Saenz De Miera, E., Ma, Y., Mo, W., Goldberg, E.M., Misumi, Y., Ikehara, Y., Neubert, T.A., et al. (2003). The CD26-related dipeptidyl aminopeptidase-like protein DPPX is a critical component of neuronal A-type K+ channels. Neuron 37, 449-461. 
Narayanan, P., Sondermann, J., Rouwette, T., Karaca, S., Urlaub, H., Mitkovski, M., Gomez-Varela, D., and Schmidt, M. (2016). Native Piezo2 interactomics identifies pericentrin as a novel regulator of Piezo2 in somatosensory neurons. J. Proteome Res. 15, 2676-2687.

Nassini, R., Materazzi, S., Benemei, S., and Geppetti, P. (2014). The TRPA1 Channel in Inflammatory and Neuropathic Pain and Migraine. (Springer, Cham), pp. 1-43.

Nesvizhskii, A.I. (2012). Computational and informatics strategies for identification of specific protein interaction partners in affinity purification mass spectrometry experiments. Proteomics 12, 1639-1655.

Niederberger, E., and Geisslinger, G. (2008). Proteomics in Neuropathic Pain Research. Anesthesiology 108, 314-323.

Nilius, B., and Flockerzi, V. (2014). Mammalian Transient Receptor Potential (TRP) Cation Channels. Volume II.

Nilius, B., and Owsianik, G. (2011). The transient receptor potential family of ion channels. Genome Biol. 12, 218.

Numazaki, M., Tominaga, T., Toyooka, H., and Tominaga, M. (2002). Direct phosphorylation of capsaicin receptor VR1 by protein kinase $\mathrm{C} \varepsilon$ and identification of two target serine residues. J. Biol. Chem. 277, 13375-13378.

Oakes, S.A., and Papa, F.R. (2015). The Role of Endoplasmic Reticulum Stress in Human Pathology. Annu. Rev. Pathol. Mech. Dis. 10, 173-194.

Offenhäuser, C., Lei, N., Roy, S., Collins, B.M., Stow, J.L., and Murray, R.Z. (2011). Syntaxin 11 binds Vtilb and regulates late endosome to lysosome fusion in macrophages. Traffic 12, 762-773.

Patapoutian, A., Tate, S., and Woolf, C.J. (2009). Transient receptor potential channels: targeting pain at the source. Nat. Rev. Drug Discov. 8, 55-68.

Peng, J., and Li, Y.J. (2010). The vanilloid receptor TRPV1: Role in cardiovascular and gastrointestinal protection. Eur. J. Pharmacol. 627, 1-7.

Perkins, J.R., Lees, J., Antunes-Martins, A., Diboun, I., McMahon, S.B., Bennett, D.L.H., and Orengo, C. (2013). PainNetworks: A web-based resource for the visualisation of painrelated genes in the context of their network associations. Pain 154, 2586.e1-2586.e12.

Pitzer, C., Kuner, R., and Tappe-Theodor, A. (2016). Voluntary and evoked behavioral correlates in inflammatory pain conditions under different social housing conditions. Pain Reports 1, e564.

Planells-Cases, R., and Ferrer-Montiel, A. (2007). TRP channel trafficking. In TRP Ion Channel Function in Sensory Transduction and Cellular Signalling Cascades, W.B. Liedtke, and S. Heller, eds. (Boca Raton: CRC Press/Taylor \& Francis), pp. 319-330.

Pomonis, J.D., Harrison, J.E., Mark, L., Bristol, D.R., Valenzano, K.J., and Walker, K. (2003). N-(4-Tertiarybutylphenyl)-4-(3-cholorphyridin-2-yl)tetrahydropyrazine -1(2H)carbox-amide (BCTC), a novel, orally effective vanilloid receptor 1 antagonist with analgesic properties: II. in vivo characterization in rat models of inflammatory and neuropath. J. Pharmacol. Exp. Ther. 306, 306.

Riley, N.M., Hebert, A.S., and Coon, J.J. (2016). Proteomics Moves into the Fast Lane. 
Cell Syst. 2, 142-143.

Ritner, H.L., Machelska, H., and Stein, C. (2009). Immune system pain and analgesia. In Science of Pain, A.I. Basbaum, and M. Bushnell, eds. (Oxford: Academic Press), pp. 407427.

Rosenbaum, T., Gordon-Shaag, A., Munari, M., and Gordon, S.E. (2004). Ca ${ }^{2+}$ /Calmodulin Modulates TRPV1 Activation by Capsaicin. J. Gen. Physiol. 123, 53-62.

Rouwette, T., Avenali, L., Sondermann, J., Narayanan, P., Gomez-Varela, D., and Schmidt, M. (2015). Modulation of nociceptive ion channels and receptors via proteinprotein interactions: Implications for pain relief. Channels 9, 175-185.

Rouwette, T., Sondermann, J., Avenali, L., Gomez-Varela, D., and Schmidt, M. (2016). Standardized profiling of the membrane-enriched proteome of mouse dorsal root ganglia (DRG) provides novel insights into chronic pain. Mol. Cell. Proteomics 15, 2152-2168.

Rowbotham, M.C., Nothaft, W., Duan, W.R., Wang, Y., Faltynek, C., McGaraughty, S., Chu, K.L., and Svensson, P. (2011). Oral and cutaneous thermosensory profile of selective TRPV1 inhibition by ABT-102 in a randomized healthy volunteer trial. Pain 152, 11921200.

Rutter, A.R., Ma, Q.-P., Leveridge, M., and Bonnert, T.P. (2005). Heteromerization and colocalization of TrpV1 and TrpV2 in mammalian cell lines and rat dorsal root ganglia. Neuroreport 16, 1735-1739.

Ryan, D.P., and Matthews, J.M. (2005). Protein-protein interactions in human disease. Curr. Opin. Struct. Biol. 15, 441-446.

Ryu, S., Liu, B., Yao, J., Fu, Q., and Qin, F. (2007). Uncoupling Proton Activation of Vanilloid Receptor TRPV1. J. Neurosci. 27, 12797-12807.

Salas, M.M., Hargreaves, K.M., and Akopian, A.N. (2009). TRPA1-mediated responses in trigeminal sensory neurons: Interaction between TRPA1 and TRPV1. Eur. J. Neurosci. 29, $1568-1578$.

Salter, M.W., and Kalia, L. V. (2004). Src kinases: a hub for NMDA receptor regulation. Nat. Rev. Neurosci. 5, 317-328.

Sanz-Salvador, L., Andrés-Borderia, A., Ferrer-Montiel, A., and Planells-Cases, R. (2012). Agonist- and Ca2+-dependent desensitization of TRPV1 channel targets the receptor to lysosomes for degradation. J. Biol. Chem. 287, 19462-19471.

Sapunar, D., Kostic, S., Banozic, A., and Puljak, L. (2012). Dorsal root ganglion - a potential new therapeutic target for neuropathic pain. J. Pain Res. 5, 31-38.

Schäfers, M., Geis, C., Svensson, C.I., Luo, Z.D., and Sommer, C. (2003). Selective increase of tumour necrosis factor-alpha in injured and spared myelinated primary afferents after chronic constrictive injury of rat sciatic nerve. Eur. J. Neurosci. 17, 791804.

Schapira, A.H. V (2012). Mitochondrial diseases. Lancet 379, 1825-1834.

Schindelin, J., Arganda-Carreras, I., Frise, E., Kaynig, V., Longair, M., Pietzsch, T., Preibisch, S., Rueden, C., Saalfeld, S., Schmid, B., et al. (2012). Fiji: An open-source platform for biological-image analysis. Nat. Methods 9, 676-682. 
Schindelin, J., Rueden, C.T., Hiner, M.C., and Eliceiri, K.W. (2015). The ImageJ ecosystem: An open platform for biomedical image analysis. Mol. Reprod. Dev. 82, 518529.

Schmidt, M., Dubin, A.E., Petrus, M.J., Earley, T.J., and Patapoutian, A. (2009).

Nociceptive signals induce trafficking of TRPA1 to the plasma membrane. Neuron 64, 498-509.

Schmidt, R., Schmelz, M., Forster, C., Ringkamp, M., Torebjiirk, E., and Handwerker, H. (1995). Novel Classes of Responsive and Unresponsive C Nociceptors in Human Skin. J. Neurosci. 15, 333-341.

Schnizler, K., Shutov, L.P., Van Kanegan, M.J., Merrill, M. a, Nichols, B., McKnight, G.S., Strack, S., Hell, J.W., and Usachev, Y.M. (2008). Protein kinase A anchoring via AKAP150 is essential for TRPV1 modulation by forskolin and prostaglandin E2 in mouse sensory neurons. J. Neurosci. 28, 4904-4917.

Schubert, O.T., Gillet, L.C., Collins, B.C., Navarro, P., Rosenberger, G., Wolski, W.E., Lam, H., Amodei, D., Mallick, P., MacLean, B., et al. (2015). Building high-quality assay libraries for targeted analysis of SWATH MS data. Nat. Protoc. 10, 426-441.

Schulte, U., Thumfart, J.O., Klöcker, N., Sailer, C.A., Bildl, W., Biniossek, M., Dehn, D., Deller, T., Eble, S., Abbass, K., et al. (2006). The epilepsy-linked Lgi1 protein assembles into presynaptic Kv1 channels and inhibits inactivation by Kvb1. Neuron 49, 697-706.

Schulte, U., Müller, C.S., and Fakler, B. (2011). Ion channels and their molecular environments - Glimpses and insights from functional proteomics. Semin. Cell Dev. Biol. $22,132-144$.

Schwanhäusser, B., Busse, D., Li, N., Dittmar, G., Schuchhardt, J., Wolf, J., Chen, W., and Selbach, M. (2011). Global quantification of mammalian gene expression control. Nature $473,337-342$.

Schwenk, J., Harmel, N., Zolles, G., Bildl, W., Kulik, A., Heimrich, B., Chisaka, O., Jonas, P., Schulte, U., Fakler, B., et al. (2009). Functional Proteomics Identify Cornichon Proteins as Auxiliary Subunits of AMPA Receptors. Science (80-. ). 323, 1313-1319.

Shevtsova, Z., Malik, J.M.I., Michel, U., Bähr, M., and Kügler, S. (2005). Promoters and serotypes: targeting of adeno-associated virus vectors for gene transfer in the rat central nervous system in vitro and in vivo. Exp. Physiol. 90, 53-59.

Shishkin, V., Potapenko, E., Kostyuk, E., Girnyk, O., Voitenko, N., and Kostyuk, P. (2002). Role of mitochondria in intracellular calcium signaling in primary and secondary sensory neurones of rats. Cell Calcium 32, 121-130.

Silva, J., Gorenstein, M., GZ, L., Vissers, J., and Geromanos, S. (2006). Absolute quantification of proteins by LCMSE: a virtue of parallel MS acquisition. Mol. Cell. Proteomics 5, 144-156.

Smith, G.D., Gunthorpe, M.J., Kelsell, R.E., Hayes, P.D., Reilly, P., Facer, P., Wright, J.E., Jerman, J.C., Walhin, J.-P., Ooi, L., et al. (2002). TRPV3 is a temperature-sensitive vanilloid receptor-like protein. Nature 418, 186-190.

Söderberg, O., Gullberg, M., Jarvius, M., Ridderstråle, K., Leuchowius, K., Jarvius, J., Wester, K., Hydbring, P., Bahram, F., Larsson, L.-G., et al. (2006). Direct observation of individual endogenous protein complexes in situ by proximity ligation. Nat. Methods 3 , 
995-1000.

Sondermann, J.R., Barry, A.M., Jahn, O., Michel, N., Abdelaziz, R., Gomez-Varela, D., and Schmidt, M. (2019). Vti1b promotes TRPV1 sensitization during inflammatory pain. Pain 160, 508-527.

Sousa-Ferreira, L., Garrido, M., Nascimento-Ferreira, I., Nobrega, C., Santos-Carvalho, A., Álvaro, A.R., Rosmaninho-Salgado, J., Kaster, M., Kügler, S., de Almeida, P.L., et al. (2011). Moderate long-term modulation of neuropeptide $\mathrm{Y}$ in hypothalamic arcuate nucleus induces energy balance alterations in adult rats. PLoS One 6, e22333.

Sousa-Valente, J., Andreou, a P., Urban, L., and Nagy, I. (2014). Transient receptor potential ion channels in primary sensory neurons as targets for novel analgesics. $\mathrm{Br}$. $\mathrm{J}$. Pharmacol. 171, 2508-2527.

Spicarova, D., Nerandzic, V., and Palecek, J. (2014). Update on the role of spinal cord TRPV1 receptors in pain modulation. Physiol. Res. 63 Suppl 1, S225-36.

Staruschenko, A., Jeske, N.A., and Akopian, A.N. (2010). Contribution of TRPV1-TRPA1 interaction to the single channel properties of the TRPA1 channel. J. Biol. Chem. 285, 15167-15177.

Stein, A.T., Ufret-Vincenty, C.A., Hua, L., Santana, L.F., and Gordon, S.E. (2006). Phosphoinositide 3-kinase binds to TRPV1 and mediates NGF-stimulated TRPV1 trafficking to the plasma membrane. J. Gen. Physiol. 128, 509-522.

Storey, J. (2002). A direct approach to false discovery rates. J. R. Stat. Soc. 64, 479-498.

Story, G.M., Peier, A.M., Reeve, A.J., Eid, S.R., Mosbacher, J., Hricik, T.R., Earley, T.J., Hergarden, A.C., Andersson, D.A., Hwang, S.W., et al. (2003). ANKTM1, a TRP-like channel expressed in nociceptive neurons, is activated by cold temperatures. Cell 112, 819-829.

Surinova, S., Radová, L., Choi, M., Srovnal, J., Brenner, H., Vitek, O., Hajdúch, M., and Aebersold, R. (2015). Non-invasive prognostic protein biomarker signatures associated with colorectal cancer. EMBO Mol Med 7, 1153-1165.

Szabo (2005). Role of Transient Receptor Potential Vanilloid 1 Receptors in AdjuvantInduced Chronic Arthritis : In Vivo Study Using Gene-Deficient Mice. Pharmacology 314, 111-119.

Szklarczyk, D., Franceschini, A., Wyder, S., Forslund, K., Heller, D., Huerta-Cepas, J., Simonovic, M., Roth, A., Santos, A., Tsafou, K.P., et al. (2015). STRING v10: Proteinprotein interaction networks, integrated over the tree of life. Nucleic Acids Res. 43, D447D452.

Szklarczyk, D., Morris, J.H., Cook, H., Kuhn, M., Wyder, S., Simonovic, M., Santos, A., Doncheva, N.T., Roth, A., Bork, P., et al. (2016). The STRING database in 2017: qualitycontrolled protein-protein association networks, made broadly accessible. Nucleic Acids Res. 39, 561-568.

Tabb, D.L., Vega-Montoto, L., Rudnick, P.A., Variyath, M.A., Ham, A.-J.L., Bunk, D.M., Kilpatrick, L.E., Billheimer, D.D., Blackman, R.K., Cardasis, H.L., et al. (2010).

Repeatability and reproducibility in proteomic identifications by liquid chromatographytandem mass spectrometry. J Proteome Res 9, 761. 
Tappe-Theodor, A., and Kuner, R. (2014). Studying ongoing and spontaneous pain in rodents - challenges and opportunities. Eur. J. Neurosci. 39, 1881-1890.

Thakur, M., Crow, M., Richards, N., Davey, G.I., Levine, E., Kelleher, J.H., Agley, C.C., Denk, F., Harridge, S.D., and McMahon, S.B. (2014). Defining the nociceptor transcriptome. Front Mol Neurosci 7, 87.

Tohda, C., Sasaki, M., Konemur, T., Sasamura, T., Itoh, M., and Kuraishi, Y. (2001). Axonal transport of VR1 capsaicin receptor mRNA in primary afferents and its participation in inflammation-induced increase in capsaicin sensitivity. J. Neurochem. 76, $1628-1635$.

Tominaga, M., Caterina, M.J., Malmberg, A.B., Rosen, T.A., Gilbert, H., Skinner, K., Raumann, B.E., Basbaum, A.I., and Julius, D. (1998). The cloned capsaicin receptor integrates multiple pain-producing stimuli. Neuron 21, 531-543.

Tóth, A., Boczán, J., Kedei, N., Lizanecz, E., Bagi, Z., Papp, Z., Édes, I., Csiba, L., and Blumberg, P.M. (2005). Expression and distribution of vanilloid receptor 1 (TRPV1) in the adult rat brain. Mol. Brain Res. 135, 162-168.

Turk, D.C., Wilson, H.D., and Cahana, A. (2011). Treatment of chronic non-cancer pain. Lancet 377, 2226-2235.

Tusher, V.G., Tibshirani, R., and Chu, G. (2001). Significance analysis of microarrays applied to the ionizing radiation response. Proc. Natl. Acad. Sci. U. S. A. 98, 5116-5121.

Tyanova, S., Temu, T., Sinitcyn, P., Carlson, A., Hein, M.Y., Geiger, T., Mann, M., and Cox, J. (2016). The Perseus computational platform for comprehensive analysis of (prote)omics data. Nat. Methods 13, 731-740.

Uçeyler, N., and Sommer, C. (2014). High-Dose Capsaicin for the Treatment of Neuropathic Pain: What We Know and What We Need to Know. Pain Ther. 3, 73.

Urban, M.J., Li, C., Yu, C., Lu, Y., Krise, J.M., McIntosh, M.P., Rajewski, R.A., Blagg, B.S.J., and Dobrowsky, R.T. (2010). Inhibiting Heat-Shock Protein 90 Reverses Sensory Hypoalgesia in Diabetic Mice. ASN Neuro 2, e00040.

Urban, M.J., Pan, P., Farmer, K.L., Zhao, H., Blagg, B.S.J., and Dobrowsky, R.T. (2012). Modulating molecular chaperones improves sensory fiber recovery and mitochondrial function in diabetic peripheral neuropathy. Exp. Neurol. 235, 388-396.

Urban, R., Scherrer, G., Goulding, E.H., Tecott, L.H., and Basbaum, A.I. (2011). Behavioral indices of ongoing pain are largely unchanged in male mice with tissue or nerve injury-induced mechanical hypersensitivity. Pain 152, 990-1000.

Usoskin, D., Furlan, A., Islam, S., Abdo, H., Lönnerberg, P., Lou, D., Hjerling-Leffler, J., Haeggström, J., Kharchenko, O., Kharchenko, P. V., et al. (2015). Unbiased classification of sensory neuron types by large-scale single-cell RNA sequencing. Nat. Neurosci. 18, $145-153$.

Vacca, V., Marinelli, S., Pieroni, L., Urbani, A., Luvisetto, S., and Pavone, F. (2014). Higher pain perception and lack of recovery from neuropathic pain in females: A behavioural, immunohistochemical, and proteomic investigation on sex-related differences in mice. Pain 155, 388-402.

Vardeh, D., Mannion, R.J., and Woolf, C.J. (2016). Toward a Mechanism-Based Approach 
to Pain Diagnosis. J. Pain 17, T50-T69.

Vellani, V., Mapplebeck, S., Moriondo, A., Davis, J.B., and McNaughton, P.A. (2001). Protein kinase $\mathrm{C}$ activation potentiates gating of the vanilloid receptor VR1 by capsaicin, protons, heat and anandamide. J. Physiol. 534, 813-825.

Vriens, J., Appendino, G., and Nilius, B. (2009). Pharmacology of Vanilloid Transient Receptor Potential Cation Channels. Mol. Pharmacol. 75, 1262-1279.

Vriens, J., Owsianik, G., Hofmann, T., Philipp, S.E., Stab, J., Chen, X., Benoit, M., Xue, F., Janssens, A., Kerselaers, S., et al. (2011). TRPM3 is a nociceptor channel involved in the detection of noxious heat. Neuron 70, 482-494.

Walker, K.M., Urban, L., Medhurst, S.J., Patel, S., Panesar, M., Fox, A.J., and McIntyre, P. (2003). The VR1 antagonist capsazepine reverses mechanical hyperalgesia in models of inflammatory and neuropathic pain. J. Pharmacol. Exp. Ther. 304, 56-62.

Ward, S.M., Bayguinov, J., Won, K.J., Grundy, D., and Berthoud, H.R. (2003).

Distribution of the vanilloid receptor (VR1) in the gastrointestinal tract. J. Comp. Neurol. 465, 121-135.

Waxman, S.G., and Zamponi, G.W. (2014). Regulating excitability of peripheral afferents: emerging ion channel targets. Nat. Neurosci. 17, 153-163.

Weng, H.J., Patel, K.N., Jeske, N.A., Bierbower, S.M., Zou, W., Tiwari, V., Zheng, Q., Tang, Z., Mo, G.C.H., Wang, Y., et al. (2015). Tmem100 Is a Regulator of TRPA1TRPV1 Complex and Contributes to Persistent Pain. Neuron 85, 833-846.

Woolf, C.J., and Ma, Q. (2007). Nociceptors--noxious stimulus detectors. Neuron 55, 353364.

Woolf, C.J., and Mannion, R.J. (1999). Neuropathic pain: aetiology, symptoms, mechanisms, and management. Lancet 353, 1959-1964.

Wu, G., Ringkamp, M., Murinson, B.B., Pogatzki, E.M., Hartke, T. V, Weerahandi, H.M., Campbell, J.N., Griffin, J.W., and Meyer, R.A. (2002). Degeneration of myelinated efferent fibers induces spontaneous activity in uninjured C-fiber afferents. J Neurosci 22, 7746-7753.

Xiong, X., Huang, S., Zhang, H., Li, J., Shen, J., Xiong, J., Lin, Y., Jiang, L., Wang, X., and Liang, S. (2009). Enrichment and proteomic analysis of plasma membrane from rat dorsal root ganglions. Proteome Sci. 7, 41.

Xu, S., Butkevich, A.N., Yamada, R., Zhou, Y., Debnatha, B., Duncana, R., Zandic, E., A., P.N., and Neamati, N. (2012). Discovery of an orally active small-molecule irreversible inhibitor of protein disulfide isomerase for ovarian cancer treatment. Proc. Natl. Acad. Sci. 109, 16348-16353.

Yu, L., Yang, F., Luo, H., Liu, F.-Y., Han, J.-S., Xing, G.-G., and Wan, Y. (2008). The role of TRPV1 in different subtypes of dorsal root ganglion neurons in rat chronic inflammatory nociception induced by complete Freund's adjuvant. Mol. Pain 4, 61 .

Zhang, E., Yi, M.-H.M.-H., Shin, N., Baek, H., Kim, S., Kim, E., Kwon, K., Lee, S., Kim, H.-W.H.-W., Chul Bae, Y., et al. (2015). Endoplasmic reticulum stress impairment in the spinal dorsal horn of a neuropathic pain model. Sci. Rep. 5, 11555.

Zhang, X., Huang, J., and McNaughton, P.A. (2005). NGF rapidly increases membrane 
expression of TRPV1 heat-gated ion channels. EMBO J. 24, 4211-4223.

Zinzalla, G., and Thurston, D.E. (2009). Targeting protein-protein interactions for therapeutic intervention: a challenge for the future. Future Med. Chem. 1, 65-93.

Zou, W., Zhan, X., Li, M., Song, Z., Liu, C., Peng, F., and Guo, Q. (2012). Identification of differentially expressed proteins in the spinal cord of neuropathic pain models with PKCgamma silence by proteomic analysis. Brain Res. 1440, 34-46. 


\section{Acknowledgement}

Without the great support that I got from so many people during the last 4 years, I could not have finished my Ph.D.: First of all, I would like to thank my two supervisors Dr. Manuela Schmidt and Dr. David Gomez-Varela. Thank you so much for taking me under your wings and letting me work in your lab. Both of you are excellent teachers and I am deeply indebted to you for all what you taught me. Thanks for your guidance and encouragement during the ups and downs of my projects and the support whenever needed. It has been a pleasure working together with you two! :)

Manuela, I especially would like to thank you for the personal help and advice you offered in times of crisis!

Furthermore, I would like to express my gratitude to the members of my thesis advisory committee, Prof. Dr. Martin Göpfert and Prof. Dr. Henning Urlaub, for their valuable input during fruitful thesis committee discussions.

Martin, special thank you for your advice and encouragement, and of course, for showing me the Seeanger for bird watching! :)

I would also like to thank Camin Dean, Ph.D., Prof. Dr. Thomas Dresbach and Prof. Dr. Michael Sereda for their willingness to serve on my extended board of examiners.

Next, I would like to acknowledge all of our collaborators for their technical support as well as helpful discussions: Dr. Olaf Jahn and Lars Piepkorn for invaluable input on imputation of missing values and MS acquisition for the TRPV1-project, Dr. Sebastian Kügler for production of viruses, and Dr. Roland Bruderer for the MS acquisition \& analysis for the DIA-MS project.

Special thanks to Sandra Drube and Prof. Dr. Michael Hörner, coordinators of the IMPRS Neurosciences program, for their assistance and support during my $\mathrm{PhD}$ journey as well as for organizing inspiring retreats.

Next, I would like to thank current and former members of the Somatosensory Signalling group for their valuable input and support on my projects: Sergej Zeiter and Tanja Nilsson - for keeping the lab running and helping with experiments. Sergej, a HUGE thank you for the countless DRG cultures you provided me with and assisting in numerous dissections. Also, thanks for hosting our lab-intern Movie Nights at your place. Pratibha Narayanan - for your support whenever needed, but above all, for the interesting and funny discussions we had. :) The two "new" PhD students, Meike Hütte - for brightening up the seemingly endless hours of animal surgeries, and Niklas Michel - for taking over the endeavour to patch TRPV1 channels for my project. Furthermore, former colleagues Dr. Luca Avenali and Dr. Elena Ciirdaeva for help on my projects. My Master students working on the TRPV1 story: Reham Abdelaziz - thank you so much for your help in the initial screening of candidates. Allison Barry - special thanks to you for your excellent and highly motivated work on my project! :) Last but not least, Dr. Tom Rouwette - for being an excellent teammate and a great friend, thank you for all your help and advice in the beginning of my $\mathrm{PhD}$ as well as now for your input on parts of my thesis. 
Moreover, I would not have made it this far if it wasn't for the support, cheer and love provided by my friends in Göttingen and elsewhere, most of all:

Marie-Theres Weil, Helene Schreyer and Martin Meschkat - thank you so much for your unconditional friendship and the amazing time we spent together!

Frankie Niwar und Heiko Dernedde - thanks for the "healthy" stress relief and the laughter we shared during Modern Arnis/ Pekiti practice sessions and seminars. Looking forward to joining you again!

Kerstin Sahlmann - for your undying support and believe in me. Thanks for being my soul sister ("Ein Herz und zwei Lungen")! (;)

Last but definitely not least: thanks to all my family, especially my aunt Joanna Voelker for continuous support and advice!

Mama \& Papa - for you undying and unconditional love and believe in me \& for supporting me in any way possible for you!!! Jan \& Jörg - thanks for all the joy, laughter but also tears and sorrow we shared! Jan, special thanks for emergency help with my stubborn computer as well as philosophical night talks! :- 


\section{Appendix}

A

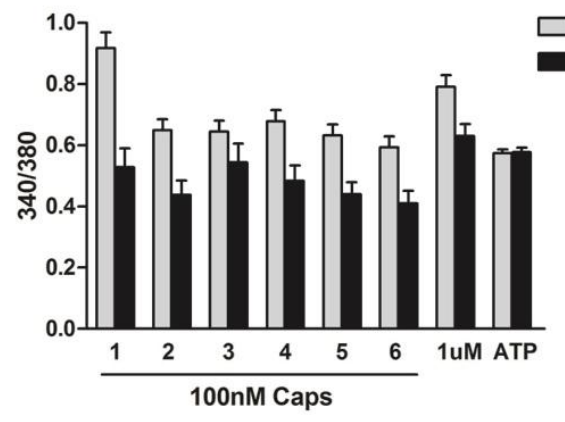

C

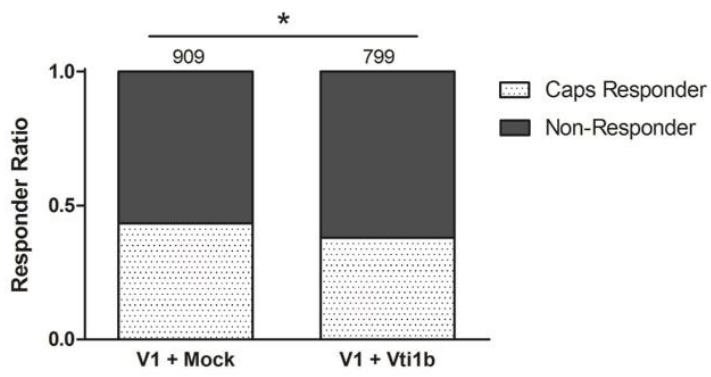

B

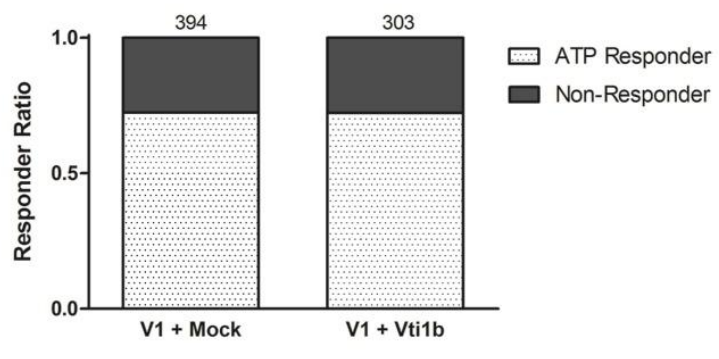

Figure 6.1: Additional information about calcium imaging experiments in HEK cells overexpressing TRPV1 + Mock or TRPV1+Vti1b

(A) Averaged response amplitudes to each capsaicin pulse (measured as peak of fluorescence absorbance ratio 340/380 i.a.u. above baseline). Response amplitudes were significantly different between TRPV1+Mock and TRPV1+Vti1b for all Caps pulses, except the $3^{\text {rd }}$ one, $* \mathrm{p}<0.05, \mathrm{~N}>173$ cells for TRPV $1+$ Mock and $\mathrm{N}>76$ for TRPV1 + Vti1b, two wayANOVA followed by uncorrected Fisher's LSD. Data represented as mean \pm SEM. (B) Proportion of cells responding to ATP as a measure of overall cellular health, ns, cell count above columns, Fisher's exact test. (C) Comparison of the number of cells responding to one or more Caps pulses (i.e. TRPV1- expressing cells) revealed a significant reduction of TRPV1-expressing cells in TRPV1 + Vti1b. TRPV1 + Mock: 0.43 vs. TRPV1 + Vti1b: $0.37, * p<0.05$, cell count above columns, Fisher's exact test. Graphs adapted with permission from A. Barry. 
A

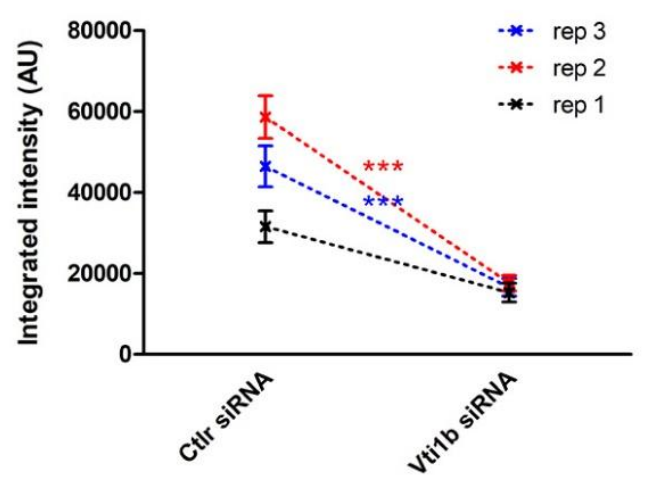

B

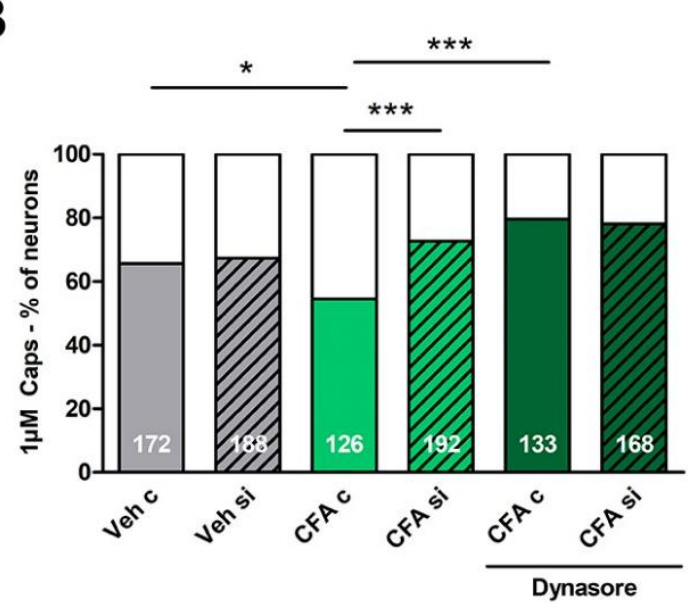

C

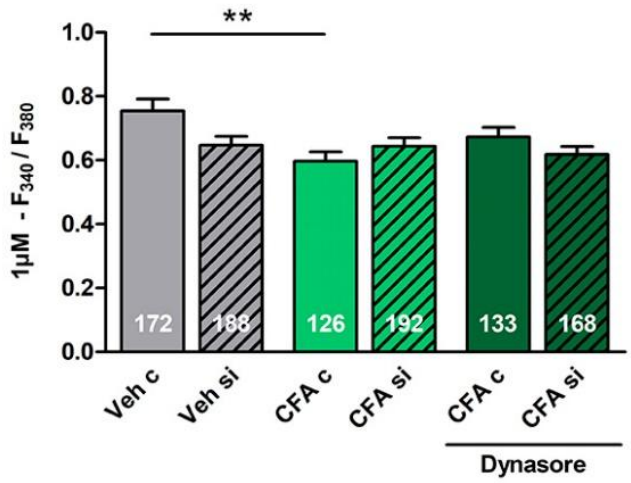

Figure 6.2: Additional information on calcium imaging experiments in IDRG neurons

(A) Quantification of Vtilb knockdown in DRG cultures. $72 \mathrm{~h}$ after transfection with control (Ctlr) or Vtilb siRNA siRNA DRG cultures were fixed and stained with antibodies against Vti1b. Apart from a decrease in positive cells (see Fig. 3.7 A ) also the integrated intensity of the Vtilb- staining is significantly decreased in the Vtilb siRNA group in 2 out of 3 independent cultures (replicate (rep) 1-3, in arbitrary units (AU)). Ctlr siRNA vs. Vti1b siRNA rep 1: ns; rep 2: $* * * \mathrm{p}<0.001$; rep 3: *** $\mathrm{p}<0.001, \mathrm{~N}>80$ neurons, $\mathrm{n}=3$ cultures, two-way ANOVA followed by Bonferroni posttests. (B) Fraction of responders (colored) and non-responders (white) to the $1 \mu \mathrm{M}$ Caps pulse given after 6x $100 \mathrm{nM}$ Caps pulses to estimate the whole TRPV1-positive population. CFA c vs. CFA si: *** p < 0.0001; Veh c vs. CFA c, Veh c: $* \mathrm{p}<0.0128$; Dynasore CFA c vs. CFA c: *** $\mathrm{p}<0.0001$, count of responders in columns, $\mathrm{n}=3-4$ independent cultures, Fisher's exact test. (C) Averaged response amplitudes (peak of fluorescence ratio 340/380 above threshold) to $1 \mu \mathrm{M}$ Caps. Veh c vs. CFA c: $* * \mathrm{p}=0.0057$, cell count in columns, $\mathrm{n}=3-4$ cultures, one-way ANOVA followed by HolmSidak's multiple comparisons test. Data represented as mean \pm SEM. Figure from Sondermann et al., 2019. 
A

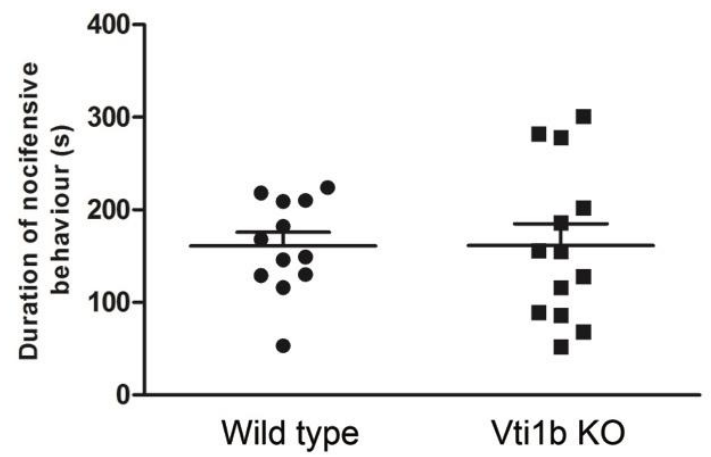

C

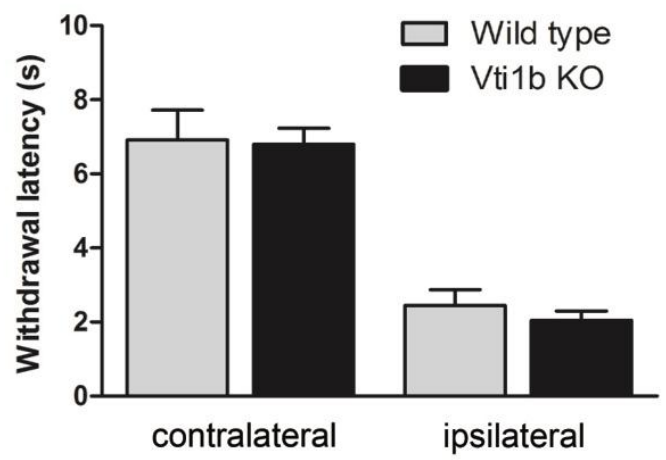

B

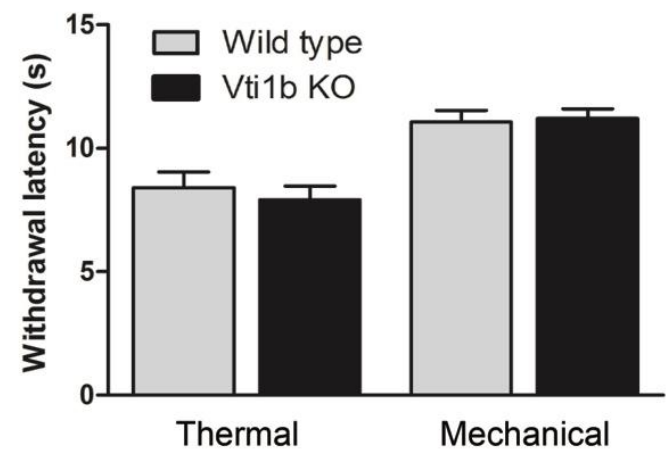

Figure 6.3: Vti1b-KO mice show normal basal sensitivity to mechanical and thermal stimuli and normal capsaicin- and CFA-induced pain behaviour

(A) Capsaicin-induced nocifensive behaviour does not differ between wild type and Vti1b KO mice. Time spent with nocifensive behaviour (licking, lifting and hiding of paw) was measured for $15 \mathrm{~min}$ after intraplantar injection of $0.5 \mu \mathrm{g}$ capsaicin, $\mathrm{ns}, \mathrm{N}=12$ mice per condition, $\mathrm{n}=3$ independent cohorts, unpaired Student's t-test. (B) Basal sensitivity to thermal or mechanical stimuli is not changed in Vtilb KO mice. Mean withdrawal latencies of hind paws to radiant heat stimulus or blunt mechanical probe did not differ significantly between genotypes, $\mathrm{ns}, \mathrm{N}=11$ mice per condition, $\mathrm{n}=2$ independent cohorts, unpaired Student's t-test. (C) Development of thermal hyperalgesia in Vtilb KO mice is similar to WT mice. Twentyfour hours after intraplantar CFA-injection the withdrawal latency to a radiant heat stimulus was assessed for both contralateral and ipsilateral hind paws. Ipsilateral paws showed significant decrease in the latency time, indicating hyperalgesia. Neither the values for the ipsilateral nor contralateral paws were significantly different between genotypes, $\mathrm{ns}, \mathrm{N}=7-9$ mice per condition, $\mathrm{n}=2$ independent cohorts, unpaired Student's t-test. Data are represented as mean \pm SEM. 
A Pretests - Thermal sensitivity
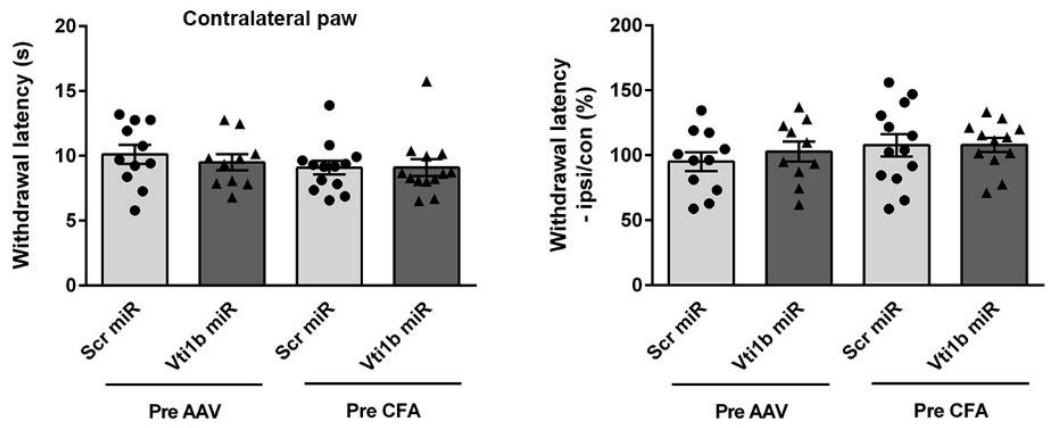

B

Pretests - Mechanical sensitivity

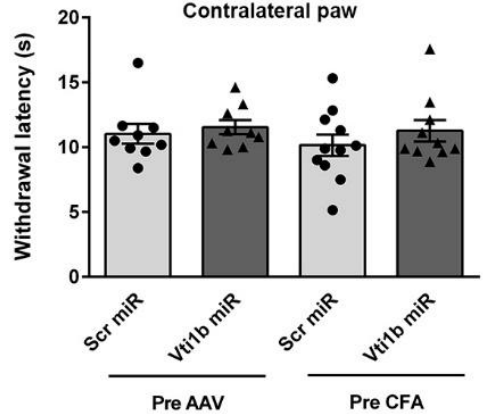

C

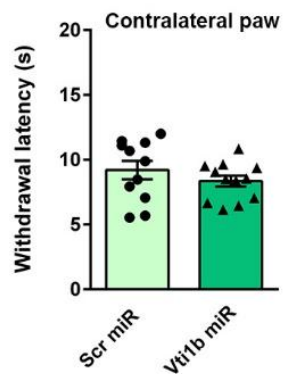

E

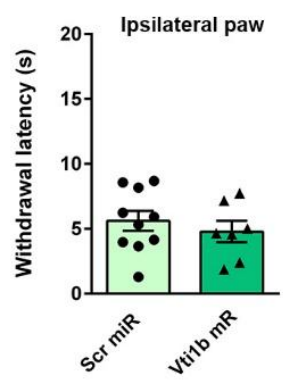

CFA 24h - Thermal sensitivity

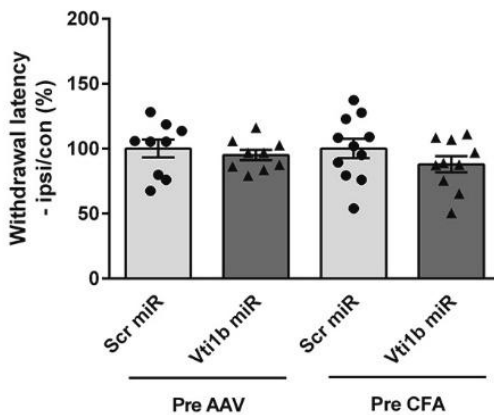

D
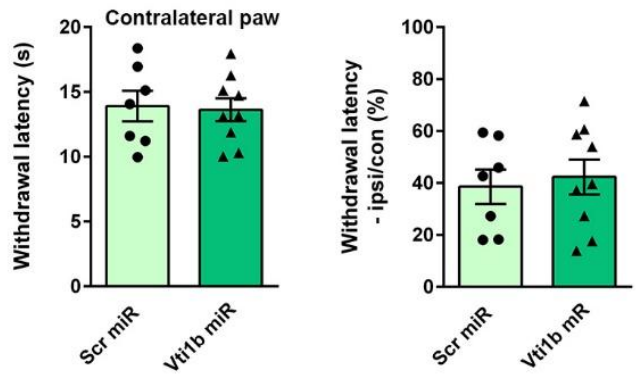

CFA 48h - Mechanical sensitivity
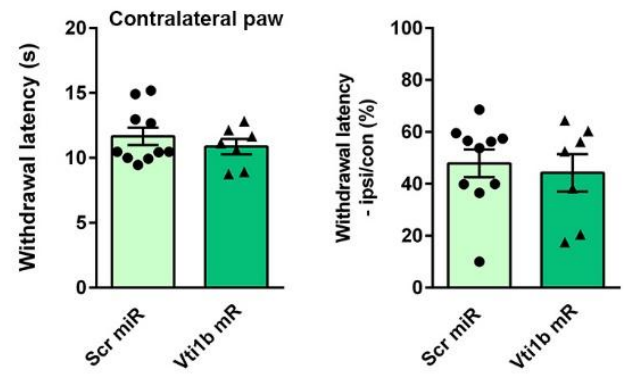


\section{Figure 6.4: Additional information on sensory behavioural tests in AAV6-Vti1b miRNA mice}

Additional information to Fig. 3.9. Knockdown of Vti1b does not change basal sensitivity to a radiant heat stimulus (A) or punctuate mechanical pressure (B), as no change in the withdrawal latency of the contralateral (con) paws (withdrawal values in seconds, left diagrams) and the normalized withdrawal latencies of injected (ipsi) to con paws (ipsi/con in \%, right diagrams). Behavioural testing took place before the virus injection (pre AAV6) and 3 weeks after the injection (pre CFA). No significant differences were determined by Kruskal-Wallis test; $\mathrm{N}>10$ mice for each group, $\mathrm{n}=2$ independent cohorts. (C) Three weeks after virus injection (either AAV6-Vtilb miR or -scramble miR) inflammatory pain was induced by CFA-injection into ipsi paw. The development of thermal hypersensitivity was assessed $24 \mathrm{~h}$ post CFA with the Hargraeves test. The left graph displays the withdrawal latencies in $\mathrm{s}$ of the non-injected con paws $(\mathrm{p}=0.3099)$ and in the right graph the normalized withdrawal latencies (\%ipsi/con, $* \mathrm{p}=0.0158)$ are shown. $\mathrm{N}=11(-12)$ mice per group, $\mathrm{n}=2$ independent cohorts. (D) Three weeks after virus injection (either AAV6-Vti1b miR or -scramble miR) inflammatory pain was induced by CFA-injection into ipsi paw. Hypersensitivity to a punctuate mechanical stimuli was assessed $24 \mathrm{~h}$ post CFA. The left graph displays the withdrawal latencies in $\mathrm{s}$ of the non-injected, con paws $(\mathrm{p}=1.0000)$ and in the right graph the normalized withdrawal latencies (\%ipsi/con, $\mathrm{p}=0.8371)$ are shown. All comparisons are $\mathrm{ns}, \mathrm{N}=7-9$ mice per group, $\mathrm{n}=2$ independent cohorts. (E, left) Three weeks after virus injection (either AAV6-Vtilb miR or -scramble miR) inflammatory pain was induced by CFA-injection into ipsi paw. Hypersensitivity of the ipsi paw to punctuate mechanical stimuli $48 \mathrm{~h}$ post CFA injection was measured as withdrawal latency in s ( $\mathrm{p}=0.5362$; same cohort tested for thermal hypersensitivity $24 \mathrm{~h}$ after CFA, Fig. 3.9). (E, middle) Withdrawal latencies of the non-injected paw to punctuate mechanical stimuli $48 \mathrm{~h}$ post CFA injection $(\mathrm{p}=0.7325)$. (E, right) Normalized withdrawal latencies of injected (ipsi) to con paws (\%ipsi/con, $p=0.7396$ ) for each individual mouse to punctuate mechanical stimuli $48 \mathrm{~h}$ post CFA injection. All comparisons $n s, N=7-10$ mice per group, $n=2$ independent cohorts. D-E: Significance was determined with the Mann Whitney test. All data are represented as mean \pm SEM. All comparisons are ns if not indicated otherwise. Figure from Sondermann, et al., 2019. 


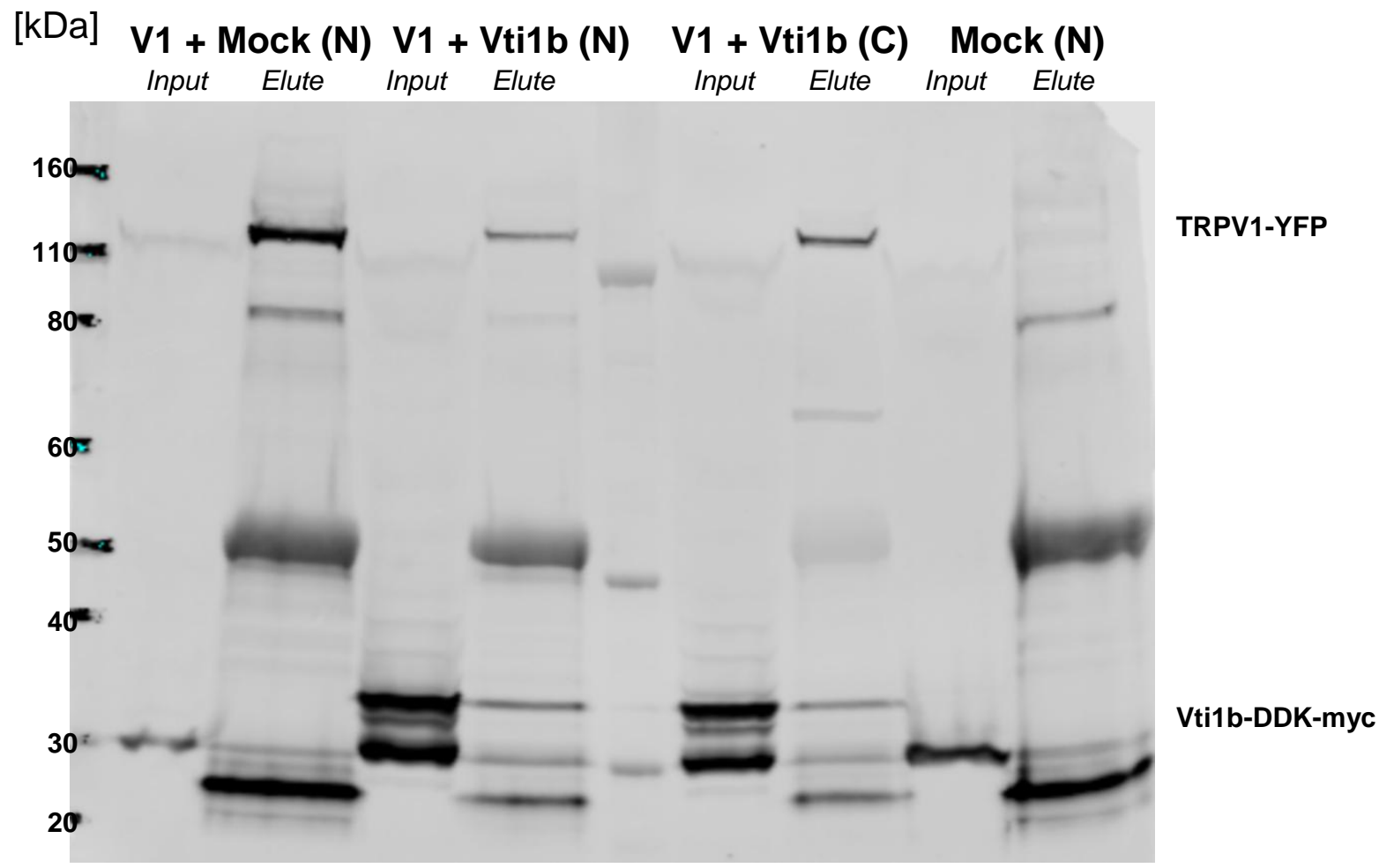

Figure 6.5: Western blot of co-immunoprecipitation of TRPV1 and Vti1b in HEK cells

Vti1b is pulled down with TRPV1 (V1) in HEK293 cells (compare Fig. 3.10 C). The protein lysate prior to the addition of an antibody is designated with 'Input'. ' $\mathrm{N}$ ' = CoIP against TRPV1 was performed with the goat $\alpha$ TRPV1 antibody targeting a N-terminal region of TRPV1. ' $\mathrm{C}$ ' = CoIP against TRPV1 was performed with the rabbit $\alpha$-TRPV1 antibody targeting a C-terminal region of TRPV1. Mock $=$ cells that were transfected with the empty pCMVSport6 plasmid. From Sondermann et al., 2019. 


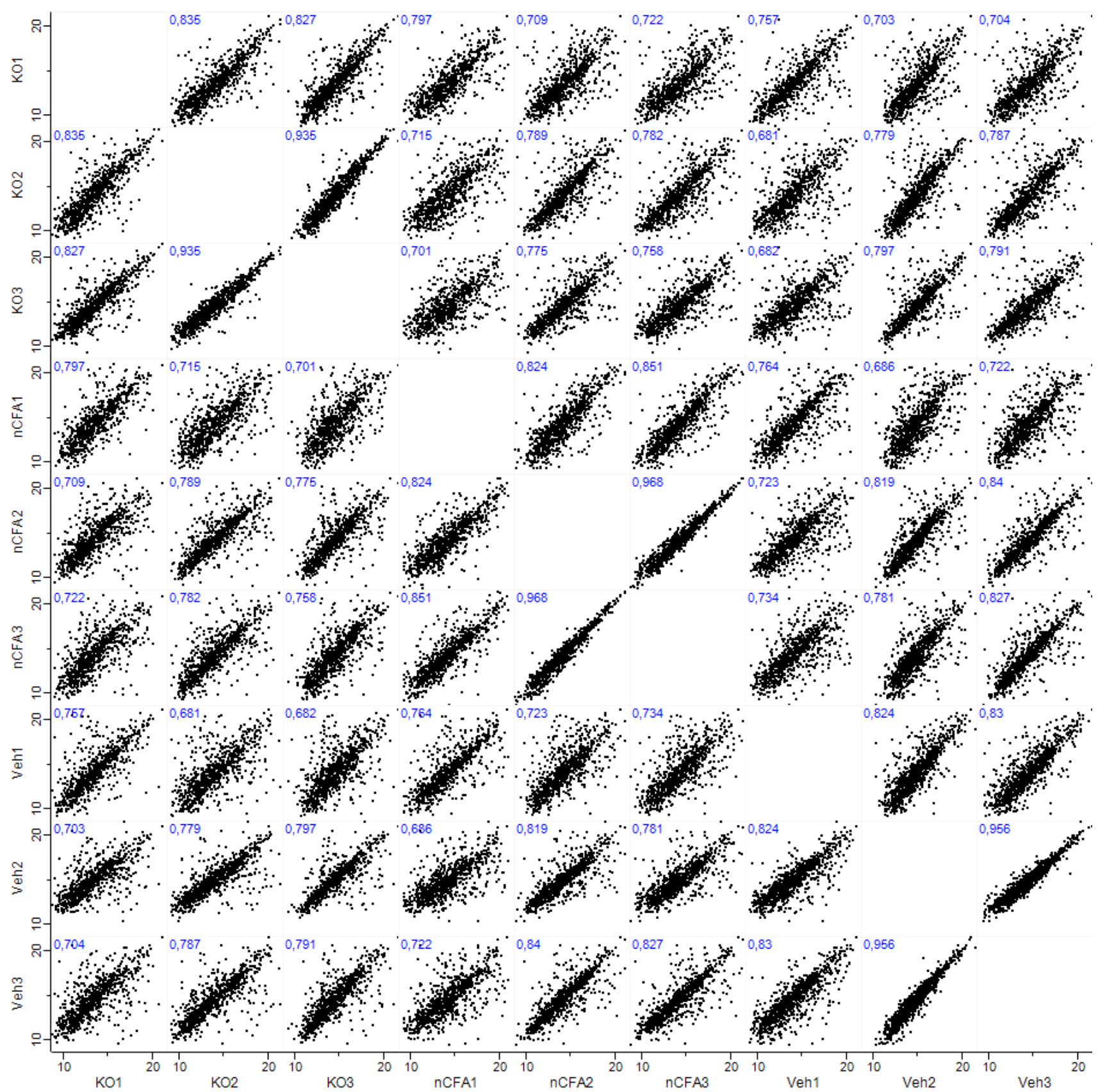

Figure 6.6: Correlation of the biological replicates between and within the groups

Multi-scatter plot of log2-transformed abundance values of all 3 biological replicates of each group (KO, Veh and nCFA). The Pearson correlation coefficient for each comparison is written in the upper left corner of each scatter plot. From Sondermann et al., 2019. 

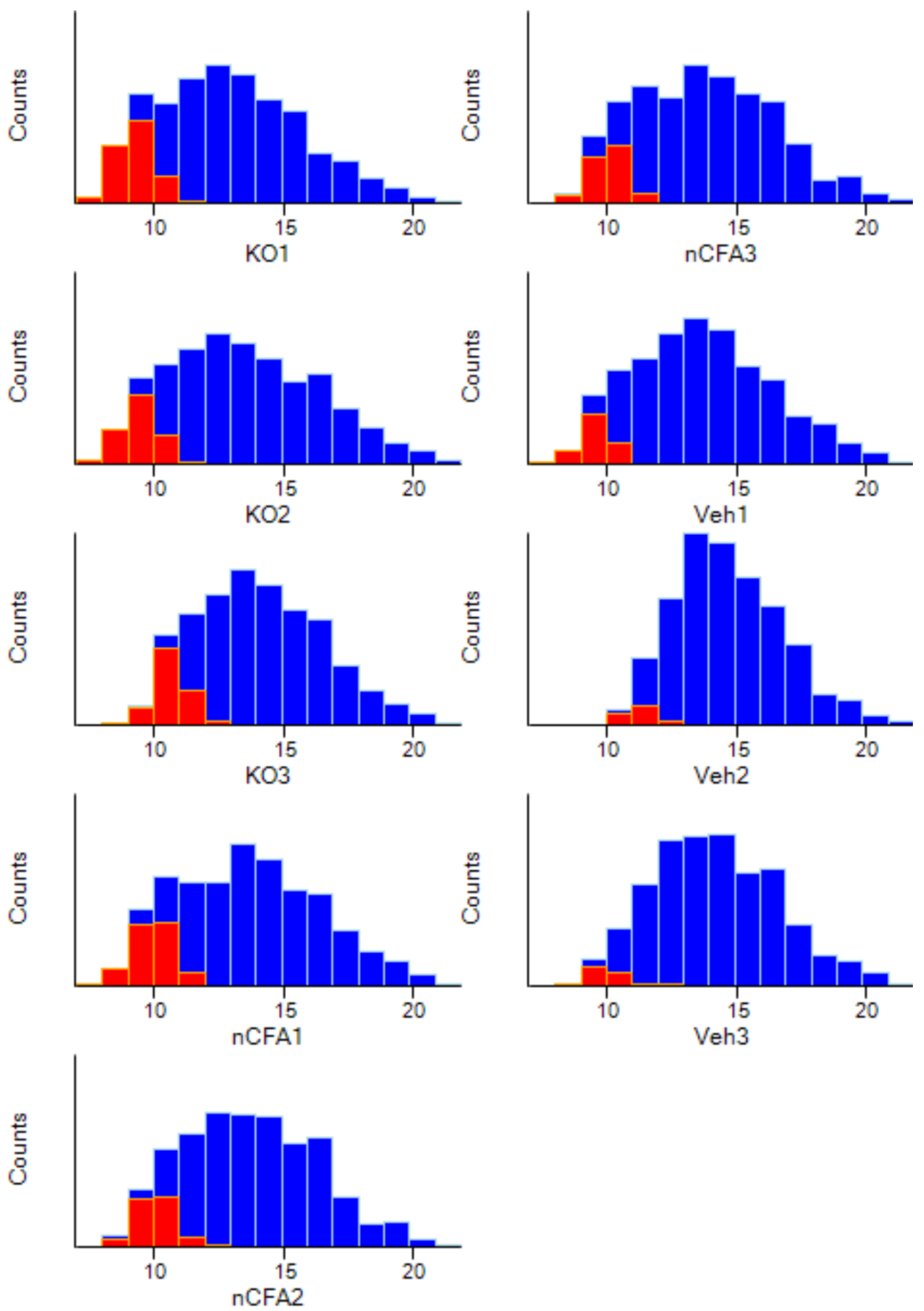

Figure 6.7: Distribution of abundance values and imputed values

Histograms show in blue the frequency distribution of detected $\log 2$ abundance values and in red the distribution of the values imputed by taking random values from a normal distribution simulating low abundance value for each of the replicates. 


\section{Table 7-1: Pain-specific interactors of TRPV1}

Putative interactors of TRPV1, i.e. proteins significantly enriched compared to KO (detected with $\log 2$ $(\mathrm{CFA} / \mathrm{KO})$ or $(\mathrm{Veh} / \mathrm{KO})>1.58$ and q-value $<0.05)$ and showing a difference of $\pm 20 \%$ between CFA and Veh $(\log 2(\mathrm{CFA} / \mathrm{Veh})>0.263$ or $<-0.263)$. T-tests were done with the missing values being imputed with random values from a normal distribution simulating low abundance values. Ribosomal and other proteins directly linked to the translation machinery as well as keratins were excluded from further analysis (last rows of this table).

\begin{tabular}{|c|c|c|c|c|c|c|}
\hline Protein name & $\begin{array}{l}\text { Uniprot } \\
\text { accession }\end{array}$ & $\begin{array}{r}\text { q-value } \\
\text { CFA/KO }\end{array}$ & $\begin{array}{c}\log 2 \\
\text { CFA/KO }\end{array}$ & $\begin{array}{l}\text { q-value } \\
\text { Veh/KO }\end{array}$ & $\begin{array}{c}\log 2 \\
\text { Veh/KO }\end{array}$ & $\begin{array}{c}\log 2 \\
\text { CFA/Veh }\end{array}$ \\
\hline Y-box-binding protein 3 & Q9JKB3 & 0,006 & 6,05 & 0,898 & $-0,28$ & 6,33 \\
\hline $\begin{array}{l}\text { Translocon-associated protein } \\
\text { subunit alpha }\end{array}$ & Q9CY50 & 0,000 & 5,51 & 0,796 & 0,56 & 4,95 \\
\hline Actin-binding LIM protein 2 & Q8BL65 & 0,000 & 5,86 & 0,599 & 0,94 & 4,92 \\
\hline Ig mu chain $\mathrm{C}$ region & P01872 & 0,000 & 4,93 & 0,980 & 0,03 & 4,90 \\
\hline $\begin{array}{l}\text { Heterogeneous nuclear } \\
\text { ribonucleoprotein } \mathrm{M}\end{array}$ & Q9D0E1 & 0,000 & 5,37 & 0,702 & 0,71 & 4,66 \\
\hline $\begin{array}{l}\text { von Willebrand factor } \mathrm{A} \\
\text { domain-containing protein } 5 \mathrm{~A}\end{array}$ & Q99KC8 & 0,000 & 5,55 & 0,408 & 1,09 & 4,46 \\
\hline $\begin{array}{l}\text { Sorting and assembly } \\
\text { machinery component } 50 \\
\text { homolog }\end{array}$ & Q8BGH2 & 0,007 & 5,07 & 0,860 & 0,67 & 4,40 \\
\hline $\begin{array}{l}\text { Farnesyl pyrophosphate } \\
\text { synthase }\end{array}$ & Q3UQN2 & 0,006 & 3,15 & 0,767 & $-0,71$ & 3,86 \\
\hline $\begin{array}{l}\text { IQ motif and SEC7 domain- } \\
\text { containing protein } 2\end{array}$ & Q5DU25 & 0,041 & 4,04 & 0,906 & 0,51 & 3,54 \\
\hline $\begin{array}{l}\text { Cysteine and glycine-rich } \\
\text { protein } 1\end{array}$ & P97315 & 0,006 & 3,29 & 0,958 & $-0,08$ & 3,37 \\
\hline $\begin{array}{l}\text { Dihydropyrimidinase-related } \\
\text { protein } 1\end{array}$ & P97427 & 0,042 & 3,12 & 0,915 & $-0,24$ & 3,36 \\
\hline Alpha-centractin & P61164 & 0,004 & 3,63 & 0,907 & 0,27 & 3,36 \\
\hline Laminin subunit gamma-1 & P02468 & 0,037 & 3,06 & 0,957 & $-0,08$ & 3,14 \\
\hline Dystonin & Q91ZU6 & 0,005 & 4,02 & 0,558 & 0,90 & 3,12 \\
\hline $\begin{array}{l}\text { Cytoplasmic FMR1-interacting } \\
\text { protein } 2\end{array}$ & Q5SQX6 & 0,044 & 2,16 & 0,620 & $-0,81$ & 2,98 \\
\hline $\begin{array}{l}\text { T-complex protein } 1 \text { subunit } \\
\text { gamma }\end{array}$ & P80318 & 0,025 & 3,16 & 0,884 & 0,37 & 2,78 \\
\hline Beta-adducin & Q9QYB8 & 0,004 & 4,28 & 0,313 & 1,62 & 2,67 \\
\hline $\begin{array}{l}\text { Nuclease-sensitive element- } \\
\text { binding protein } 1\end{array}$ & P62960 & 0,036 & 3,22 & 0,724 & 0,65 & 2,56 \\
\hline Ras-related protein Rab-8B & P61028 & 0,006 & 2,97 & 0,812 & 0,52 & 2,44 \\
\hline
\end{tabular}




\begin{tabular}{|c|c|c|c|c|c|c|}
\hline $\begin{array}{l}\text { Hemoglobin subunit epsilon- } \\
\text { Y2 }\end{array}$ & P02104 & 0,006 & 3,21 & 0,686 & 0,77 & 2,44 \\
\hline $\begin{array}{l}\text { Somatomedin-B and } \\
\text { thrombospondin type-1 } \\
\text { domain-containing protein }\end{array}$ & Q3UPR9 & 0,040 & 2,82 & 0,798 & 0,50 & 2,32 \\
\hline Collagen alpha-1(VI) chain & Q04857 & 0,003 & 4,29 & 0,135 & 2,00 & 2,29 \\
\hline Kinectin & Q61595 & 0,016 & 4,05 & 0,124 & 2,14 & 1,91 \\
\hline Filamin-B & Q80X90 & 0,003 & 4,61 & 0,019 & 2,99 & 1,62 \\
\hline Protein flightless-1 homolog & Q9JJ28 & 0,035 & 2,44 & 0,669 & 0,83 & 1,60 \\
\hline Unconventional myosin-Id & Q5SYD0 & 0,006 & 2,71 & 0,410 & 1,21 & 1,50 \\
\hline $\begin{array}{l}\text { LIM domain and actin-binding } \\
\text { protein } 1\end{array}$ & Q9ERG0 & 0,006 & 3,07 & 0,128 & 1,71 & 1,35 \\
\hline Lumican & P51885 & 0,027 & 2,77 & 0,441 & 1,49 & 1,28 \\
\hline $\begin{array}{l}\text { F-actin-capping protein subunit } \\
\text { beta }\end{array}$ & P47757 & 0,006 & 3,03 & 0,111 & 1,85 & 1,19 \\
\hline Catalase & P24270 & 0,000 & 9,11 & 0,000 & 8,10 & 1,01 \\
\hline Vinculin & Q64727 & 0,006 & 5,89 & 0,000 & 4,90 & 0,99 \\
\hline Fibrinogen alpha chain & E9PV24 & 0,003 & 4,21 & 0,004 & 3,29 & $\overline{0,92}$ \\
\hline Ras-related protein Rab-11B & P46638 & 0,003 & 3,51 & 0,040 & 2,64 & 0,87 \\
\hline $\begin{array}{l}\text { F-actin-capping protein subunit } \\
\text { alpha-1 }\end{array}$ & P47753 & 0,028 & 2,52 & 0,145 & 1,73 & 0,80 \\
\hline Ras-related protein Rab-6A & P35279 & 0,047 & 2,59 & 0,110 & 1,85 & 0,73 \\
\hline Twinfilin-1 & Q91YR1 & 0,006 & 2,69 & 0,149 & 1,96 & 0,73 \\
\hline $\begin{array}{l}\text { Microtubule-associated } \\
\text { proteins 1A/1B light chain 3A }\end{array}$ & Q91VR7 & 0,023 & 2,63 & 0,263 & 1,94 & 0,70 \\
\hline Ras-related protein Ral-A & P63321 & 0,004 & 4,97 & 0,001 & 4,43 & 0,54 \\
\hline $\begin{array}{l}\text { Serine/threonine-protein kinase } \\
\text { MARK2 }\end{array}$ & Q05512 & 0,004 & 4,13 & 0,000 & 3,67 & 0,46 \\
\hline $\begin{array}{l}\text { Signal recognition particle } \\
\text { receptor subunit beta }\end{array}$ & P47758 & 0,028 & 2,54 & 0,110 & 2,09 & 0,45 \\
\hline $\begin{array}{l}\text { Transient receptor potential } \\
\text { cation channel subfamily V } \\
\text { member } 2\end{array}$ & Q9WTR1 & 0,005 & 4,35 & 0,000 & 3,90 & 0,45 \\
\hline $\begin{array}{l}\text { Splicing factor U2AF } 65 \mathrm{kDa} \\
\text { subunit }\end{array}$ & P26369 & 0,006 & 3,59 & 0,020 & 3,19 & 0,40 \\
\hline Coronin-2A & Q8C0P5 & 0,035 & 2,24 & 0,083 & 1,88 & 0,36 \\
\hline $\begin{array}{l}\text { Apoptosis-inducing factor } 1 \text {, } \\
\text { mitochondrial }\end{array}$ & Q9Z0X1 & 0,006 & 2,84 & 0,046 & 2,57 & 0,27 \\
\hline
\end{tabular}




\begin{tabular}{|c|c|c|c|c|c|c|}
\hline RNA-binding protein 14 & Q8C2Q3 & 0,006 & 3,45 & 0,001 & 3,74 & $-0,30$ \\
\hline $\begin{array}{l}\text { Vesicle transport through } \\
\text { interaction with t-SNAREs } \\
\text { homolog 1B }\end{array}$ & O88384 & 0,004 & 4,11 & 0,000 & 4,43 & $-0,32$ \\
\hline Cytospin-A & Q2KN98 & 0,006 & 2,87 & 0,003 & 3,21 & $-0,33$ \\
\hline Unconventional myosin-If & P70248 & 0,123 & 2,64 & 0,019 & 3,02 & $-0,38$ \\
\hline Dynactin subunit 1 & O08788 & 0,003 & 3,49 & 0,000 & 3,88 & $-0,39$ \\
\hline $\begin{array}{l}\text { Lysocardiolipin acyltransferase } \\
1\end{array}$ & Q3UN02 & 0,087 & 2,38 & 0,046 & 2,80 & $-0,42$ \\
\hline Clathrin light chain B & Q6IRU5 & 0,006 & 4,54 & 0,001 & 5,02 & $-0,48$ \\
\hline $\begin{array}{l}\text { Cytoplasmic dynein } 1 \text { light } \\
\text { intermediate chain } 2\end{array}$ & Q6PDL0 & 0,006 & 3,06 & 0,003 & 3,57 & $-0,51$ \\
\hline $\begin{array}{l}\text { TSC22 domain family protein } \\
4\end{array}$ & Q9EQN3 & 0,045 & 2,28 & 0,013 & 2,81 & $-0,52$ \\
\hline Ataxin-2 & O70305 & 0,092 & 2,94 & 0,014 & 3,52 & $-0,59$ \\
\hline Actin-binding LIM protein 1 & Q8K4G5 & 0,086 & 2,31 & 0,030 & 2,91 & $-0,60$ \\
\hline Ras-related protein Rap-1A & P62835 & 0,003 & 3,48 & 0,001 & 4,09 & $-0,60$ \\
\hline $\begin{array}{l}\text { Staphylococcal nuclease } \\
\text { domain-containing protein } 1\end{array}$ & Q78PY7 & 0,027 & 2,46 & 0,007 & 3,12 & $-0,67$ \\
\hline Ataxin-2-like protein & Q7TQH0 & 0,006 & 3,38 & 0,000 & 4,14 & $-0,76$ \\
\hline $\begin{array}{l}\text { Probable ATP-dependent RNA } \\
\text { helicase DDX6 }\end{array}$ & P54823 & 0,070 & 2,43 & 0,003 & 3,21 & $-0,77$ \\
\hline Histone H1.3 & P43277 & 0,007 & 4,94 & 0,000 & 5,73 & $-0,79$ \\
\hline Protein LSM12 homolog & Q9D0R8 & 0,025 & 3,62 & 0,001 & 4,47 & $-0,85$ \\
\hline AP-3 complex subunit delta-1 & O54774 & 0,084 & 1,93 & 0,004 & 2,78 & $-0,85$ \\
\hline $\begin{array}{l}\text { Pleckstrin homology domain- } \\
\text { containing family A member } 6\end{array}$ & Q7TQG1 & 0,006 & 5,40 & 0,000 & 6,29 & $-0,89$ \\
\hline Lactoylglutathione lyase & Q9CPU0 & 0,124 & 2,14 & 0,031 & 3,03 & $-0,90$ \\
\hline ATP-citrate synthase & Q91V92 & 0,011 & 2,75 & 0,005 & 3,67 & $-0,92$ \\
\hline Thy-1 membrane glycoprotein & P01831 & 0,127 & 2,04 & 0,038 & 3,00 & $-0,96$ \\
\hline Alpha-adducin & Q9QYC0 & 0,006 & 3,40 & 0,000 & 4,38 & $-0,98$ \\
\hline Muscleblind-like protein 1 & Q9JKP5 & 0,004 & 4,33 & 0,000 & 5,41 & $-1,08$ \\
\hline $\begin{array}{l}\text { Dedicator of cytokinesis } \\
\text { protein } 11\end{array}$ & A2AF47 & 0,021 & 2,96 & 0,000 & 4,16 & $-1,19$ \\
\hline Unconventional myosin-Ie & E9Q634 & 0,187 & 1,38 & 0,031 & 2,76 & $-1,38$ \\
\hline Coatomer subunit gamma-1 & Q9QZE5 & 0,008 & 3,10 & 0,000 & 4,50 & $-1,40$ \\
\hline Nexilin & Q7TPW1 & 0,080 & 1,96 & 0,005 & 3,40 & $-1,44$ \\
\hline
\end{tabular}




\begin{tabular}{|c|c|c|c|c|c|c|}
\hline $\begin{array}{l}\text { Translationally-controlled } \\
\text { tumor protein }\end{array}$ & P63028 & 0,285 & 1,44 & 0,019 & 2,95 & $-1,51$ \\
\hline $\begin{array}{l}\text { Proteasome subunit alpha type- } \\
6\end{array}$ & Q9QUM9 & 0,113 & 1,89 & 0,004 & 3,44 & $-1,55$ \\
\hline $\begin{array}{l}\text { Spectrin alpha chain, } \\
\text { erythrocytic } 1\end{array}$ & P08032 & 0,437 & 1,24 & 0,015 & 2,85 & $-1,61$ \\
\hline Tubulin beta- 1 chain & A2AQ07 & 0,471 & 1,10 & 0,001 & 3,19 & $-2,10$ \\
\hline Nck-associated protein 1 & P28660 & 0,908 & 0,31 & 0,048 & 2,73 & $-2,42$ \\
\hline Partner of Y14 and mago & Q8CHP5 & 0,277 & 1,33 & 0,000 & 3,87 & $-2,54$ \\
\hline $\begin{array}{l}\text { Heat shock protein HSP 90- } \\
\text { alpha }\end{array}$ & P07901 & 0,832 & 0,41 & 0,004 & 3,00 & $-2,59$ \\
\hline AP-2 complex subunit alpha-2 & P17427 & 0,954 & 0,14 & 0,008 & 2,74 & $-2,60$ \\
\hline Proteasome subunit beta type- 1 & O09061 & 0,957 & $-0,15$ & 0,035 & 2,56 & $-2,72$ \\
\hline Parvalbumin alpha & P32848 & 0,796 & 0,56 & 0,002 & 3,47 & $-2,91$ \\
\hline Filamin-A & Q8BTM8 & 0,770 & 0,56 & 0,000 & 3,71 & $-3,15$ \\
\hline $\begin{array}{l}\text { 2-oxoglutarate dehydrogenase, } \\
\text { mitochondrial }\end{array}$ & Q60597 & 0,966 & 0,09 & 0,004 & 3,40 & $-3,30$ \\
\hline $\begin{array}{l}\text { Sarcoplasmic/endoplasmic } \\
\text { reticulum calcium ATPase } 2\end{array}$ & O55143 & 0,999 & 0,00 & 0,001 & 3,33 & $-3,33$ \\
\hline $\begin{array}{l}\text { LIM and SH3 domain protein } \\
1\end{array}$ & Q61792 & 0,924 & $-0,25$ & 0,002 & 3,11 & $-3,36$ \\
\hline $\begin{array}{l}\text { V-type proton ATPase subunit } \\
\text { E } 1\end{array}$ & P50518 & 0,929 & $-0,25$ & 0,004 & 3,18 & $-3,43$ \\
\hline Protein RUFY3 & Q9D394 & 0,904 & 0,26 & 0,000 & 3,71 & $-3,45$ \\
\hline Unconventional myosin-VIIa & P97479 & 0,728 & $-0,75$ & 0,014 & 2,80 & $-3,54$ \\
\hline $\begin{array}{l}\text { Peptidyl-prolyl cis-trans } \\
\text { isomerase FKBP1A }\end{array}$ & P26883 & 0,983 & 0,06 & 0,003 & 3,72 & $-3,66$ \\
\hline Collagen alpha-1(XIV) chain & Q80X19 & 0,820 & $-0,43$ & 0,001 & 3,49 & $-3,92$ \\
\hline $\begin{array}{l}\text { Cold shock domain-containing } \\
\text { protein E1 }\end{array}$ & Q91W50 & 0,887 & $-0,31$ & 0,002 & 3,65 & $-3,96$ \\
\hline $\begin{array}{l}\text { RNA-binding motif protein, } \mathrm{X} \\
\text { chromosome }\end{array}$ & Q9WV02 & 0,836 & 0,54 & 0,000 & 4,75 & $-4,21$ \\
\hline $\begin{array}{l}\text { Nascent polypeptide- } \\
\text { associated complex subunit } \\
\text { alpha, muscle-specific form }\end{array}$ & P70670 & 0,007 & 3,69 & 0,000 & 7,93 & $-4,25$ \\
\hline $\begin{array}{l}\text { ADP-ribosylation factor-like } \\
\text { protein } 8 \mathrm{~A}\end{array}$ & Q8VEH3 & 0,874 & $-0,43$ & 0,002 & 3,95 & $-4,38$ \\
\hline Cysteine-rich protein 2 & Q9DCT8 & 0,723 & 0,72 & 0,000 & 5,12 & $-4,40$ \\
\hline
\end{tabular}




\begin{tabular}{|c|c|c|c|c|c|c|}
\hline $\begin{array}{l}\text { BMP-2-inducible protein } \\
\text { kinase }\end{array}$ & Q91Z96 & 0,833 & $-0,52$ & 0,002 & 3,99 & $-4,51$ \\
\hline Band 4.1-like protein 3 & Q9WV92 & 0,122 & 1,72 & 0,000 & 6,24 & $-4,52$ \\
\hline $\begin{array}{l}\text { ATPase family AAA domain- } \\
\text { containing protein } 1\end{array}$ & Q9D5T0 & 0,683 & $-0,66$ & 0,001 & 3,89 & $-4,55$ \\
\hline $\begin{array}{l}\text { LIM and calponin homology } \\
\text { domains-containing protein } 1\end{array}$ & Q3UH68 & 0,000 & 5,81 & 0,000 & 10,43 & $-4,62$ \\
\hline $\begin{array}{l}\text { Peptidyl-prolyl cis-trans } \\
\text { isomerase FKBP2 }\end{array}$ & P45878 & 0,796 & $-0,56$ & 0,004 & 4,07 & $-4,64$ \\
\hline $\begin{array}{l}\text { DnaJ homolog subfamily C } \\
\text { member } 9\end{array}$ & Q91WN1 & 0,966 & $-0,11$ & 0,000 & 4,69 & $-4,79$ \\
\hline $\begin{array}{l}\text { Oxysterol-binding protein- } \\
\text { related protein } 3\end{array}$ & Q9DBS9 & 0,655 & 0,80 & 0,000 & 5,80 & $-5,00$ \\
\hline Cathepsin B & P10605 & 0,759 & $-0,82$ & 0,002 & 4,56 & $-5,38$ \\
\hline $\begin{array}{l}\text { NADH dehydrogenase } \\
\text { [ubiquinone] } 1 \text { beta } \\
\text { subcomplex subunit } 10\end{array}$ & Q9DCS9 & 0,990 & $-0,02$ & 0,000 & 5,53 & $-5,55$ \\
\hline $\begin{array}{l}\text { Serine/threonine-protein } \\
\text { phosphatase } 2 \mathrm{~A} 65 \mathrm{kDa} \\
\text { regulatory subunit A alpha } \\
\text { isoform }\end{array}$ & Q76MZ3 & 0,004 & $-3,06$ & 0,045 & 2,52 & $-5,58$ \\
\hline Septin-2 & P42208 & 0,634 & 0,70 & 0,000 & 6,33 & $-5,64$ \\
\hline $\begin{array}{l}\text { Mitogen-activated protein } \\
\text { kinase } 1\end{array}$ & P63085 & 0,620 & $-0,90$ & 0,000 & 4,86 & $-5,77$ \\
\hline $\begin{array}{l}\text { ATP-dependent 6- } \\
\text { phosphofructokinase, muscle } \\
\text { type }\end{array}$ & P47857 & 0,926 & 0,21 & 0,000 & 6,15 & $-5,94$ \\
\hline $\begin{array}{l}\text { ATP-dependent 6- } \\
\text { phosphofructokinase, platelet } \\
\text { type }\end{array}$ & $\begin{array}{l}\text { Q9WUA } \\
3\end{array}$ & 0,761 & 0,57 & 0,000 & 6,54 & $-5,97$ \\
\hline Alpha-actinin-2 & Q9JI91 & 0,971 & $-0,10$ & 0,000 & 5,95 & $-6,05$ \\
\hline Integrin alpha-6 & Q61739 & 0,830 & 0,54 & 0,000 & 6,78 & $-6,24$ \\
\hline Coatomer subunit beta & Q9JIF7 & 0,124 & $-1,85$ & 0,000 & 4,45 & $-6,30$ \\
\hline Plasmolipin & Q9DCU2 & 0,905 & $-0,34$ & 0,000 & 6,21 & $-6,55$ \\
\hline $\begin{array}{l}\text { Charged multivesicular body } \\
\text { protein } 4 \mathrm{~b}\end{array}$ & Q9D8B3 & 0,928 & $-0,35$ & 0,000 & 7,16 & $-7,51$ \\
\hline $\begin{array}{l}\text { SH3 domain-binding glutamic } \\
\text { acid-rich-like protein }\end{array}$ & Q9JJU8 & 0,466 & $-0,99$ & 0,000 & 6,57 & $-7,56$ \\
\hline
\end{tabular}


Excluded proteins (Ribosome \&

Translation, Keratin)

\begin{tabular}{|c|c|c|c|c|c|c|}
\hline $\begin{array}{l}\text { 39S ribosomal protein } \mathrm{L} 11 \text {, } \\
\text { mitochondrial }\end{array}$ & Q9CQF0 & 0,059 & 2,34 & 0,000 & 5,58 & $-3,23$ \\
\hline $40 \mathrm{~S}$ ribosomal protein $\mathrm{S} 2$ & P25444 & 0,004 & 3,46 & 0,896 & 0,25 & 3,21 \\
\hline 40S ribosomal protein $\mathrm{S} 27$ & Q6ZWU9 & 0,006 & 3,04 & 0,000 & 3,63 & $-0,59$ \\
\hline 40S ribosomal protein $\mathrm{S} 27$-like & Q6ZWY3 & 0,016 & 2,95 & 0,001 & 3,88 & $-0,93$ \\
\hline 60S ribosomal protein L38 & Q9JJI8 & 0,801 & 0,49 & 0,002 & 3,24 & $-2,74$ \\
\hline $\begin{array}{l}\text { Aminoacyl tRNA synthase } \\
\text { complex-interacting } \\
\text { multifunctional protein } 2\end{array}$ & Q8R010 & 0,258 & 2,26 & 0,039 & 3,29 & $-1,04$ \\
\hline $\begin{array}{l}\text { ATP-dependent RNA helicase } \\
\text { DDX3Y }\end{array}$ & Q62095 & 0,000 & 6,92 & 0,000 & 4,57 & 2,35 \\
\hline $\begin{array}{l}\text { Bifunctional } \\
\text { glutamate/proline--tRNA } \\
\text { ligase }\end{array}$ & Q8CGC7 & 0,005 & 3,80 & 0,698 & 0,69 & 3,11 \\
\hline $\begin{array}{l}\text { Eukaryotic translation } \\
\text { initiation factor } 2 \text { subunit } 3, \mathrm{X} \text { - } \\
\text { linked }\end{array}$ & Q9Z0N1 & 0,906 & 0,38 & 0,001 & 4,13 & $-3,75$ \\
\hline $\begin{array}{l}\text { Eukaryotic translation } \\
\text { initiation factor } 3 \text { subunit B }\end{array}$ & Q8JZQ9 & 0,062 & 2,93 & 0,008 & 3,67 & $-0,74$ \\
\hline $\begin{array}{l}\text { Eukaryotic translation } \\
\text { initiation factor } 3 \text { subunit } \mathrm{C}\end{array}$ & Q8R1B4 & 0,018 & 2,29 & 0,004 & 2,74 & $-0,45$ \\
\hline $\begin{array}{l}\text { Eukaryotic translation } \\
\text { initiation factor } 4 \text { gamma } 3\end{array}$ & Q80XI3 & 0,004 & 5,11 & 0,000 & 5,58 & $-0,47$ \\
\hline $\begin{array}{l}\text { Isoleucine--tRNA ligase, } \\
\text { cytoplasmic }\end{array}$ & Q8BU30 & 0,006 & 2,69 & 0,531 & 0,90 & 1,79 \\
\hline Lysine--tRNA ligase & Q99MN1 & 0,028 & 2,58 & 0,723 & 0,78 & 1,80 \\
\hline $\begin{array}{l}\text { Phenylalanine--tRNA ligase } \\
\text { beta subunit }\end{array}$ & $\begin{array}{c}\text { Q9WUA } \\
2\end{array}$ & 0,000 & 7,03 & 0,938 & $-0,11$ & 7,14 \\
\hline Ribosome-binding protein 1 & Q99PL5 & 0,077 & 2,61 & 0,000 & 4,79 & $-2,18$ \\
\hline Keratin, type I cytoskeletal 28 & A6BLY7 & 0,949 & 0,19 & 0,000 & 5,22 & \\
\hline
\end{tabular}




\section{Table 7-2: Putative TRPV1 interactors not detected in the KO group}

Proteins listed in this table were not detected in the $\mathrm{KO}$ at all, but not declared as significantly regulated in CFA vs. KO or Veh vs. KO, by the t-test due to the missing value imputation via normal distribution. Log2 (CFA/Veh) denotes the $\log 2$ fold change of these proteins, or states whether the protein was only found in CFA- or the Veh group (no fold change calculation possible).

\begin{tabular}{|c|c|c|}
\hline Protein name & $\begin{array}{l}\text { Uniprot } \\
\text { accession }\end{array}$ & $\begin{array}{c}\log 2 \\
\text { CFA/Veh }\end{array}$ \\
\hline Transmembrane protein 263 & Q9DAM7 & only CFA \\
\hline Proteasome subunit beta type- 3 & Q9R1P1 & only CFA \\
\hline Ras-related protein Rab-8A & P55258 & only CFA \\
\hline Myosin-6 & Q02566 & only CFA \\
\hline Ras-related protein Rap-2c & Q8BU31 & only CFA \\
\hline Rho-related GTP-binding protein RhoB & P62746 & only CFA \\
\hline Eukaryotic translation initiation factor $5 \mathrm{~A}-2$ & Q8BGY2 & only CFA \\
\hline ADP/ATP translocase 4 & Q3V132 & only CFA \\
\hline Tubulin alpha-8 chain & Q9JJZ2 & only CFA \\
\hline Ras-related protein Rab-8B & P61028 & 2,44 \\
\hline Filamin-B & Q80X90 & 1,62 \\
\hline Microtubule-associated proteins 1A/1B light chain 3A & Q91VR7 & 1,24 \\
\hline Catalase & P24270 & 1,01 \\
\hline Vinculin & Q64727 & 0,99 \\
\hline Fibrinogen alpha chain & E9PV24 & 0,92 \\
\hline Ras-related protein Rab-11B & P46638 & 0,87 \\
\hline Syntaxin-8 & O88983 & 0,76 \\
\hline Ras-related protein Rab-6A & P35279 & 0,73 \\
\hline Twinfilin-1 & Q91YR1 & 0,73 \\
\hline Ras-related protein Ral-A & P63321 & 0,54 \\
\hline Dynein light chain roadblock-type 1 & P62627 & 0,47 \\
\hline Serine/threonine-protein kinase MARK2 & Q05512 & 0,46 \\
\hline Signal recognition particle receptor subunit beta & P47758 & 0,45 \\
\hline $\begin{array}{l}\text { Transient receptor potential cation channel subfamily } \mathrm{V} \\
\text { member } 2\end{array}$ & Q9WTR1 & 0,45 \\
\hline Splicing factor U2AF $65 \mathrm{kDa}$ subunit & P26369 & 0,40 \\
\hline
\end{tabular}




\begin{tabular}{|c|c|c|}
\hline Dephospho-CoA kinase domain-containing protein & Q8BHC4 & 0,27 \\
\hline Apoptosis-inducing factor 1 , mitochondrial & Q9Z0X1 & 0,27 \\
\hline Double zinc ribbon and ankyrin repeat-containing protein 1 & Q8C008 & $-0,28$ \\
\hline Kinesin heavy chain isoform $5 \mathrm{C}$ & P28738 & $-0,31$ \\
\hline $26 \mathrm{~S}$ proteasome non-ATPase regulatory subunit 13 & Q9WVJ2 & $-0,35$ \\
\hline BAG family molecular chaperone regulator 2 & Q91YN9 & $-0,37$ \\
\hline Growth hormone-inducible transmembrane protein & Q91VC9 & $-0,38$ \\
\hline 39S ribosomal protein L14, mitochondrial & Q9D1I6 & $-0,45$ \\
\hline Mapk-regulated corepressor-interacting protein 1 & Q3UGS4 & $-0,49$ \\
\hline Mitotic checkpoint protein BUB3 & Q9WVA3 & $-0,49$ \\
\hline Gap junction alpha-1 protein & P23242 & $-0,54$ \\
\hline 26S proteasome non-ATPase regulatory subunit 14 & O35593 & $-0,66$ \\
\hline Nucleoside diphosphate kinase B & Q01768 & $-0,74$ \\
\hline General vesicular transport factor p115 & Q9Z1Z0 & $-0,77$ \\
\hline Myosin light chain 4 & P09541 & $-0,80$ \\
\hline Kinesin-1 heavy chain & Q61768 & $-0,89$ \\
\hline Kinesin heavy chain isoform $5 \mathrm{~A}$ & $\mathrm{P} 33175$ & $-0,94$ \\
\hline Chloride intracellular channel protein 1 & Q9Z1Q5 & $-0,96$ \\
\hline Heat shock $70 \mathrm{kDa}$ protein 4 & Q61316 & $-1,06$ \\
\hline Calcium-binding protein 39 & Q06138 & $-1,19$ \\
\hline Protein-L-isoaspartate(D-aspartate) O-methyltransferase & P23506 & $-1,30$ \\
\hline Clathrin light chain A & O08585 & $-1,89$ \\
\hline $26 \mathrm{~S}$ proteasome non-ATPase regulatory subunit 2 & Q8VDM4 & only Veh \\
\hline 3-hydroxyisobutyryl-CoA hydrolase, mitochondrial & Q8QZS1 & only Veh \\
\hline Adenylate kinase isoenzyme 1 & Q9R0Y5 & only Veh \\
\hline Alpha-synuclein & O55042 & only Veh \\
\hline ATP synthase subunit $\mathrm{g}$, mitochondrial & Q9CPQ8 & only Veh \\
\hline ATP synthase-coupling factor 6 , mitochondrial & P97450 & only Veh \\
\hline Calmegin & P52194 & only Veh \\
\hline Calpain small subunit 1 & O88456 & only Veh \\
\hline Cellular nucleic acid-binding protein & P53996 & only Veh \\
\hline
\end{tabular}




\begin{tabular}{|c|c|c|}
\hline Cytochrome c oxidase subunit 5A, mitochondrial & P12787 & only Veh \\
\hline Dehydrogenase/reductase SDR family member 7 & Q9CXR1 & only Veh \\
\hline Endoplasmic reticulum resident protein 29 & P57759 & only Veh \\
\hline Erlin-1 & Q91X78 & only Veh \\
\hline ES1 protein homolog, mitochondrial & Q9D172 & only Veh \\
\hline Ferritin light chain 1 & P29391 & only Veh \\
\hline Golgi-associated plant pathogenesis-related protein 1 & Q9CYL5 & only Veh \\
\hline $\begin{array}{l}\text { Guanine nucleotide-binding protein } \mathrm{G}(\mathrm{I}) / \mathrm{G}(\mathrm{S}) / \mathrm{G}(\mathrm{O}) \text { subunit } \\
\text { gamma-2 }\end{array}$ & P63213 & only Veh \\
\hline Guanine nucleotide-binding protein subunit alpha-14 & P30677 & only Veh \\
\hline Heat shock $70 \mathrm{kDa}$ protein $4 \mathrm{~L}$ & P48722 & only Veh \\
\hline Heme oxygenase 2 & O70252 & only Veh \\
\hline Inorganic pyrophosphatase & Q9D819 & only Veh \\
\hline Isovaleryl-CoA dehydrogenase, mitochondrial & Q9JHI5 & only Veh \\
\hline LanC-like protein 1 & O89112 & only Veh \\
\hline Microtubule cross-linking factor 1 & Q3UHU5 & only Veh \\
\hline Microtubule-associated protein RP/EB family member 3 & Q6PER3 & only Veh \\
\hline NADH dehydrogenase [ubiquinone] 1 subunit $\mathrm{C} 2$ & Q9CQ54 & only Veh \\
\hline $\begin{array}{l}\text { NADH dehydrogenase [ubiquinone] flavoprotein } 2 \text {, } \\
\text { mitochondrial }\end{array}$ & Q9D6J6 & only Veh \\
\hline Prefoldin subunit 2 & O70591 & only Veh \\
\hline Proteasome subunit alpha type- 5 & Q9Z2U1 & only Veh \\
\hline Proteasome subunit beta type- 5 & O55234 & only Veh \\
\hline Protein deglycase DJ-1 & Q99LX0 & only Veh \\
\hline Protein lin-7 homolog $\mathrm{C}$ & O88952 & only Veh \\
\hline Protein S100-A13 & P97352 & only Veh \\
\hline Protein sel-1 homolog 1 & Q9Z2G6 & only Veh \\
\hline Rab GTPase-binding effector protein 1 & O35551 & only Veh \\
\hline $\begin{array}{l}\text { Serine/threonine-protein phosphatase } 2 \mathrm{~B} \text { catalytic subunit } \\
\text { alpha isoform }\end{array}$ & P63328 & only Veh \\
\hline SLIT-ROBO Rho GTPase-activating protein 3 & Q812A2 & only Veh \\
\hline Small nuclear ribonucleoprotein $\mathrm{E}$ & P62305 & only Veh \\
\hline
\end{tabular}




\begin{tabular}{|l|c|c|} 
Superoxide dismutase $[\mathrm{Mn}]$, mitochondrial & P09671 & only Veh \\
\hline Trans-2-enoyl-CoA reductase, mitochondrial & Q9DCS3 & only Veh \\
\hline Translocating chain-associated membrane protein 1 & Q91V04 & only Veh \\
\hline Ubiquitin carboxyl-terminal hydrolase 5 & P56399 & only Veh \\
\hline UMP-CMP kinase & Q9DBP5 & only Veh \\
\hline X-ray repair cross-complementing protein 6 & P23475 & only Veh \\
\hline
\end{tabular}

Excluded proteins (Ribosome \& Translation)

\begin{tabular}{|l|c|c|}
\hline 60S ribosomal protein L38 & Q9JJI8 & only Veh \\
\hline Eukaryotic translation initiation factor 2 subunit 3, X-linked & Q9Z0N1 & only Veh \\
\hline Eukaryotic translation initiation factor 4 gamma 3 & Q80XI3 & $-0,47$ \\
\hline Eukaryotic translation initiation factor 3 subunit C & Q8R1B4 & $-0,45$ \\
\hline Eukaryotic translation initiation factor 3 subunit B & Q8JZQ9 & $-0,74$ \\
\hline Keratin, type I cytoskeletal 27 & Q9Z320 & only Veh \\
\hline Keratin, type I cytoskeletal 28 & A6BLY7 & only Veh \\
\hline
\end{tabular}




\section{Table 7-3: Enriched annotation term clusters for Veh-enriched proteins and proteins only detected in Veh}

Functional annotation clustering tool from DAVID was used to determine enriched annotation terms (see section 2.1.7.2 for further details) GO-Categories: CC, cellular component; MF, molecular function, BP, biological process; P-Value, significance of gene-term enrichment with a modified Fisher's exact test; Proteins, uniprot_ID of proteins linked to the respective GO term; Fold enrichment (FEn), magnitude of enrichment comparing the enrichment of genes in input list vs. background (whole mouse genome); Benjamini (Benj), global correction of individual p-values for multiples testing. In bold writing Vtilb (O88384).

\begin{tabular}{|c|c|c|c|c|c|c|}
\hline $\begin{array}{l}\text { Annotation } \\
\text { Cluster } 1\end{array}$ & Enrichment Score: 5.7 & & & & & \\
\hline Category & Term & $\%$ & P-Value & Proteins & FEn & Benj \\
\hline MF & $\begin{array}{l}\text { GO:0098641 cadherin } \\
\text { binding involved in cell-cell } \\
\text { adhesion }\end{array}$ & 10,4 & $1,05 \mathrm{E}-07$ & $\begin{array}{l}\text { Q9D8B3, Q9WUA3, Q61792, } \\
\text { Q7TQH0, P54823, P42208, } \\
\text { Q9QYC0, Q61739, Q9Z1Z0, } \\
\text { Q9Z1Q5, Q99LX0, Q61768, } \\
\text { Q78PY7, Q8BTM8 }\end{array}$ & 6,9 & $3,13 \mathrm{E}-05$ \\
\hline $\mathrm{CC}$ & $\begin{array}{l}\text { GO:0005913 cell-cell } \\
\text { adherens junction }\end{array}$ & 10,4 & $1,65 \mathrm{E}-07$ & $\begin{array}{l}\text { Q9D8B3, Q9WUA3, Q61792, } \\
\text { Q7TQH0, P54823, P42208, } \\
\text { Q9QYC0, Q61739, Q9Z1Z0, } \\
\text { Q9Z1Q5, Q99LX0, Q61768, } \\
\text { Q78PY7, Q8BTM8 }\end{array}$ & 6,7 & $1,24 \mathrm{E}-05$ \\
\hline $\mathrm{BP}$ & $\begin{array}{l}\text { GO:0098609 cell-cell } \\
\text { adhesion }\end{array}$ & 5,9 & 4,37E-04 & $\begin{array}{l}\text { Q9Z1Z0, Q9WUA3, Q61792, } \\
\text { Q78PY7, Q7TQH0, P54823, } \\
\text { P42208, Q9QYC0 }\end{array}$ & 5,8 & 0,097 \\
\hline $\begin{array}{l}\text { Annotation } \\
\text { Cluster } 2\end{array}$ & Enrichment Score: 3.4 & & & & & \\
\hline Category & Term & $\%$ & P-Value & Proteins & FEn & Benj \\
\hline $\mathrm{BP}$ & $\begin{array}{l}\text { GO:0016192 vesicle- } \\
\text { mediated transport }\end{array}$ & 7,4 & $2,37 \mathrm{E}-05$ & $\begin{array}{l}\text { Q9Z1Z0, O54774, Q9JIF7, } \\
\text { P17427, Q9QZE5, O08585, } \\
\text { Q6IRU5, O88384, P33175, } \\
\text { O35551 }\end{array}$ & 6,5 & 0,022 \\
\hline $\mathrm{CC}$ & $\begin{array}{l}\text { GO:0030117 membrane } \\
\text { coat }\end{array}$ & 3,7 & $4,25 \mathrm{E}-05$ & $\begin{array}{l}\text { O54774, Q9JIF7, Q9D8B3, } \\
\text { P17427, Q9QZE5 }\end{array}$ & 25,2 & 0,002 \\
\hline $\mathrm{BP}$ & $\begin{array}{l}\text { GO:0006886 intracellular } \\
\text { protein transport }\end{array}$ & 7,4 & $4,63 \mathrm{E}-05$ & $\begin{array}{l}\text { Q9Z1Z0, O54774, Q9JIF7, } \\
\text { P57759, P17427, Q9QZE5, } \\
\text { O08585, Q6IRU5, P97479, } \\
\mathbf{0 8 8 3 8 4}\end{array}$ & 5,9 & 0,021 \\
\hline $\mathrm{BP}$ & $\begin{array}{l}\text { GO:0015031 protein } \\
\text { transport }\end{array}$ & 9,6 & 0,001 & $\begin{array}{l}\text { Q9Z1Z0, O54774, Q9JIF7, } \\
\text { O88952, Q9D8B3, P17427, } \\
\text { Q9QZE5, Q91V04, P70670, } \\
\text { P62835, O88384, P97352, } \\
\text { O35551 }\end{array}$ & 3,0 & 0,144 \\
\hline $\mathrm{CC}$ & $\begin{array}{l}\text { GO:0000139 Golgi } \\
\text { membrane }\end{array}$ & 3,7 & 0,273 & $\begin{array}{l}\text { Q9Z1Z0, O54774, Q9JIF7, } \\
\text { Q9CYL5, Q9QZE5 }\end{array}$ & 1,9 & 0,771 \\
\hline & & & & & & \\
\hline
\end{tabular}




\begin{tabular}{|c|c|c|c|c|c|c|}
\hline $\begin{array}{l}\text { Annotation } \\
\text { Cluster } 3\end{array}$ & Enrichment Score: 2.6 & & & & & \\
\hline Category & Term & $\%$ & P-Value & Proteins & FEn & Benj \\
\hline $\mathrm{CC}$ & $\begin{array}{l}\text { GO:0000502 proteasome } \\
\text { complex }\end{array}$ & 5,2 & 4,67E-06 & $\begin{array}{l}\text { O35593, Q9Z2U1, O55234, } \\
\text { Q9QUM9, Q8VDM4, O09061, } \\
\text { Q9WVJ2 }\end{array}$ & 16,0 & $2,34 \mathrm{E}-04$ \\
\hline MF & $\begin{array}{l}\text { GO:0004175 endopeptidase } \\
\text { activity }\end{array}$ & 5,9 & $1,59 \mathrm{E}-05$ & $\begin{array}{l}\text { P10605, O35593, Q9Z2U1, } \\
\text { O55234, Q9QUM9, Q8VDM4, } \\
\text { O09061, Q9WVJ2 }\end{array}$ & 9,9 & 0,002 \\
\hline $\mathrm{CC}$ & $\begin{array}{l}\text { GO:0005839 proteasome } \\
\text { core complex }\end{array}$ & 3,0 & $2,90 \mathrm{E}-04$ & $\begin{array}{l}\text { Q9Z2U1, O55234, Q9QUM9, } \\
\text { O09061 }\end{array}$ & 30,2 & 0,007 \\
\hline MF & $\begin{array}{l}\text { GO:0004298 threonine- } \\
\text { type endopeptidase activity }\end{array}$ & 3,0 & $5,73 \mathrm{E}-04$ & $\begin{array}{l}\text { Q9Z2U1, O55234, Q9QUM9, } \\
\text { O09061 }\end{array}$ & 24,1 & 0,024 \\
\hline $\mathrm{BP}$ & $\begin{array}{l}\text { GO:0051603 proteolysis } \\
\text { involved in cellular protein } \\
\text { catabolic process }\end{array}$ & 3,7 & $7,50 \mathrm{E}-04$ & $\begin{array}{l}\text { P10605, Q9Z2U1, O55234, } \\
\text { Q9QUM9, O09061 }\end{array}$ & 12,1 & 0,130 \\
\hline $\mathrm{BP}$ & $\begin{array}{l}\text { GO:0002479 antigen } \\
\text { processing and presentation } \\
\text { of exogenous peptide } \\
\text { antigen via MHC class I, } \\
\text { TAP-dependent }\end{array}$ & 3,0 & 0,001 & $\begin{array}{l}\text { Q9Z2U1, O55234, Q9QUM9, } \\
\text { O09061 }\end{array}$ & 18,4 & 0,138 \\
\hline $\mathrm{CC}$ & $\begin{array}{l}\text { GO:0022624 proteasome } \\
\text { accessory complex }\end{array}$ & 2,2 & 0,005 & O35593, Q8VDM4, Q9WVJ2 & 26,7 & 0,079 \\
\hline $\mathrm{BP}$ & GO:0006508 proteolysis & 6,7 & 0,059 & $\begin{array}{l}\text { P10605, O35593, Q99LX0, } \\
\text { Q9Z2U1, O55234, Q9QUM9, } \\
\text { O88456, P56399, O09061 }\end{array}$ & 2,1 & 0,915 \\
\hline MF & $\begin{array}{l}\text { GO:0008233 peptidase } \\
\text { activity }\end{array}$ & 5,9 & 0,078 & $\begin{array}{l}\text { P10605, O35593, Q99LX0, } \\
\text { Q9Z2U1, O55234, Q9QUM9, } \\
\text { P56399, O09061 }\end{array}$ & 2,1 & 0,565 \\
\hline MF & $\begin{array}{l}\text { GO:0016787 hydrolase } \\
\text { activity }\end{array}$ & 11,9 & 0,134 & $\begin{array}{l}\text { P23475, Q9Z2U1, O55234, } \\
\text { P54823, P56399, P50518, } \\
\text { O09061, P63328, P10605, } \\
\text { O55143, O35593, Q8QZS1, } \\
\text { Q99LX0, Q9D819, Q9QUM9, } \\
\text { Q9D5T0 }\end{array}$ & 1,4 & 0,727 \\
\hline BP & $\begin{array}{l}\text { GO:0043161 proteasome- } \\
\text { mediated ubiquitin- } \\
\text { dependent protein catabolic } \\
\text { process }\end{array}$ & 2,2 & 0,291 & Q9Z2U1, O55234, Q8VDM4 & 2,8 & 0,998 \\
\hline $\begin{array}{l}\text { Annotation } \\
\text { Cluster } 4\end{array}$ & Enrichment Score: 2.1 & & & & & \\
\hline Category & Term & $\%$ & P-Value & Proteins & FEn & Benj \\
\hline MF & GO:0003774 motor activity & 3,7 & 0,002 & $\begin{array}{l}\text { Q6PDL0, E9Q634, O08788, } \\
\text { P97479, P70248 }\end{array}$ & 8,8 & 0,065 \\
\hline $\mathrm{CC}$ & $\begin{array}{l}\text { GO:0016459 myosin } \\
\text { complex }\end{array}$ & 3,0 & 0,005 & $\begin{array}{l}\text { P09541, E9Q634, P97479, } \\
\text { P70248 }\end{array}$ & 11,6 & 0,074 \\
\hline
\end{tabular}


Appendix | 128

MF 


\section{Table 7-4: Enriched annotation term clusters for CFA-enriched proteins and proteins only detected in CFA}

Functional annotation clustering tool from DAVID was used to determine enriched annotation terms (see section 2.1.7.2 for further details) GO-Categories: CC, cellular component; MF, molecular function, BP, biological process; P-Value, significance of gene-term enrichment with a modified Fisher's exact test; Proteins, uniprot_ID of proteins linked to the respective GO term; Fold enrichment (FEn), magnitude of enrichment comparing the enrichment of genes in input list vs. background (whole mouse genome); Benjamini (Benj), global correction of individual p-values for multiples testing.

\begin{tabular}{|c|c|c|c|c|c|c|}
\hline $\begin{array}{l}\text { Annotation } \\
\text { Cluster } 1\end{array}$ & Enrichment Score: 8.0 & & & & & \\
\hline Category & Term & $\%$ & P-Value & Proteins & FEn & Benj \\
\hline MF & GO:0003779 actin binding & 23,2 & $2,37 \mathrm{E}-10$ & $\begin{array}{l}\text { Q91YR1, Q8BL65, P47753, } \\
\text { Q9ERG0, Q02566, P47757, } \\
\text { Q80X90, Q9QYB8, Q8C0P5, } \\
\text { Q91ZU6, Q64727, Q5SYD0, } \\
\text { Q9JJ28 }\end{array}$ & 12,7 & $3,13 \mathrm{E}-08$ \\
\hline $\mathrm{CC}$ & GO:0005903 brush border & 14,3 & $1,87 \mathrm{E}-09$ & $\begin{array}{l}\text { P47753, Q9ERG0, P47757, } \\
\text { Q80X90, Q8C0P5, Q64727, } \\
\text { Q5SYD0, Q9JJ28 }\end{array}$ & 36,5 & $3,60 \mathrm{E}-07$ \\
\hline MF & $\begin{array}{l}\text { GO:0051015 actin filament } \\
\text { binding }\end{array}$ & 12,5 & $2,56 \mathrm{E}-06$ & $\begin{array}{l}\text { P47753, Q9ERG0, P47757, } \\
\text { Q9QYB8, Q8C0P5, Q64727, } \\
\text { Q5SYD0 }\end{array}$ & 17,5 & $1,13 \mathrm{E}-04$ \\
\hline $\begin{array}{l}\text { Annotation } \\
\text { Cluster } 2\end{array}$ & Enrichment Score: 4.7 & & & & & \\
\hline Category & Term & $\%$ & P-Value & Proteins & FEn & Benj \\
\hline MF & $\begin{array}{l}\text { GO:0051015 actin filament } \\
\text { binding }\end{array}$ & 12,5 & $2,56 \mathrm{E}-06$ & $\begin{array}{l}\text { P47753, Q9ERG0, P47757, } \\
\text { Q9QYB8, Q8C0P5, Q64727, } \\
\text { Q5SYD0 }\end{array}$ & 17,5 & $1,13 \mathrm{E}-04$ \\
\hline $\mathrm{BP}$ & $\begin{array}{l}\text { GO:0051016 barbed-end } \\
\text { actin filament capping }\end{array}$ & 7,1 & $1,22 \mathrm{E}-05$ & $\begin{array}{l}\text { Q91YR1, P47753, P47757, } \\
\text { Q9QYB8 }\end{array}$ & 85,3 & 0,001 \\
\hline $\mathrm{CC}$ & $\begin{array}{l}\text { GO:0008290 F-actin } \\
\text { capping protein complex }\end{array}$ & 5,4 & $2,73 \mathrm{E}-04$ & P47753, P47757, Q9QYB8 & 117,0 & 0,006 \\
\hline $\begin{array}{l}\text { Annotation } \\
\text { Cluster } 3\end{array}$ & Enrichment Score: 4.1 & & & & & \\
\hline Category & Term & $\%$ & P-Value & Proteins & FEn & Benj \\
\hline $\mathrm{CC}$ & GO:0005903 brush border & 14,3 & $1,87 \mathrm{E}-09$ & $\begin{array}{l}\text { P47753, Q9ERG0, P47757, } \\
\text { Q80X90, Q8C0P5, Q64727, } \\
\text { Q5SYD0, Q9JJ28 }\end{array}$ & 36,5 & $3,60 \mathrm{E}-07$ \\
\hline MF & $\begin{array}{l}\text { GO:0098641 cadherin } \\
\text { binding involved in cell-cell } \\
\text { adhesion }\end{array}$ & 14,3 & $1,79 \mathrm{E}-05$ & $\begin{array}{l}\text { Q91YR1, P47753, Q9ERG0, } \\
\text { P46638, P47757, Q80X90, } \\
\text { Q64727, Q05512 }\end{array}$ & 9,4 & $3,95 \mathrm{E}-04$ \\
\hline $\mathrm{CC}$ & $\begin{array}{l}\text { GO:0005913 cell-cell } \\
\text { adherens junction }\end{array}$ & 14,3 & $2,71 \mathrm{E}-05$ & $\begin{array}{l}\text { Q91YR1, P47753, Q9ERG0, } \\
\text { P46638, P47757, Q80X90, } \\
\text { Q64727, Q05512 }\end{array}$ & 8,9 & $8,73 \mathrm{E}-04$ \\
\hline BP & $\begin{array}{l}\text { GO:0098609 cell-cell } \\
\text { adhesion }\end{array}$ & 10,7 & $2,06 \mathrm{E}-04$ & $\begin{array}{l}\text { Q91YR1, P47753, Q9ERG0, } \\
\text { P47757, Q80X90, Q05512 }\end{array}$ & 10,8 & 0,018 \\
\hline
\end{tabular}




\begin{tabular}{|c|c|c|c|c|c|c|}
\hline $\mathrm{CC}$ & GO:0005884 actin filament & 5,4 & 0,016 & P47757, Q64727, Q05512 & 15,5 & 0,123 \\
\hline $\mathrm{CC}$ & $\begin{array}{l}\text { GO:0005911 cell-cell } \\
\text { junction }\end{array}$ & 5,4 & 0,109 & Q91YR1, P47753, Q64727 & 5,2 & 0,434 \\
\hline $\begin{array}{l}\text { Annotation } \\
\text { Cluster } 4\end{array}$ & Enrichment Score: 2.9 & & & & & \\
\hline Category & Term & $\%$ & P-Value & Proteins & FEn & Benj \\
\hline MF & GO:0019003 GDP binding & 10,7 & $9,32 \mathrm{E}-07$ & $\begin{array}{l}\text { P62746, P55258, Q8BU31, } \\
\text { P61028, P46638, P63321 }\end{array}$ & 32,9 & $6,15 \mathrm{E}-05$ \\
\hline $\mathrm{BP}$ & $\begin{array}{l}\text { GO:0007264 small GTPase } \\
\text { mediated signal transduction }\end{array}$ & 14,3 & 4,79E-06 & $\begin{array}{l}\text { P62746, P55258, Q8BU31, } \\
\text { P61028, P35279, P46638, } \\
\text { P63321, P47758 }\end{array}$ & 11,6 & $8,33 \mathrm{E}-04$ \\
\hline MF & GO:0005525 GTP binding & 16,1 & $1,62 \mathrm{E}-05$ & $\begin{array}{l}\text { Q9JJZ2, P62746, P55258, } \\
\text { Q8BU31, P61028, P35279, } \\
\text { P46638, P63321, P47758 }\end{array}$ & 7,7 & $4,28 \mathrm{E}-04$ \\
\hline MF & $\begin{array}{l}\text { GO:0000166 nucleotide } \\
\text { binding }\end{array}$ & 30,4 & $1,04 \mathrm{E}-04$ & $\begin{array}{l}\text { Q9JJZ2, P62746, P55258, } \\
\text { P61028, P35279, Q02566, } \\
\text { P63321, P26369, Q8BHC4, } \\
\text { P80318, Q9D0E1, Q8BU31, } \\
\text { P46638, P47758, P61164, } \\
\text { Q5SYD0, Q05512 }\end{array}$ & 2,9 & 0,002 \\
\hline MF & $\begin{array}{l}\text { GO:0003924 GTPase } \\
\text { activity }\end{array}$ & 8,9 & 0,003 & $\begin{array}{l}\text { Q9JJZ2, P55258, P35279, } \\
\text { P46638, P63321 }\end{array}$ & 7,9 & 0,050 \\
\hline $\mathrm{CC}$ & $\begin{array}{l}\text { GO:0055037 recycling } \\
\text { endosome }\end{array}$ & 7,1 & 0,004 & $\begin{array}{l}\text { P55258, Q8BU31, P46638, } \\
\text { O88983 }\end{array}$ & 12,1 & 0,053 \\
\hline $\mathrm{CC}$ & $\begin{array}{l}\text { GO:0045335 phagocytic } \\
\text { vesicle }\end{array}$ & 5,4 & 0,006 & P55258, P61028, P46638 & 25,7 & 0,065 \\
\hline $\mathrm{CC}$ & $\begin{array}{l}\text { GO:0055038 recycling } \\
\text { endosome membrane }\end{array}$ & 5,4 & 0,006 & P55258, Q8BU31, P46638 & 24,5 & 0,067 \\
\hline $\mathrm{BP}$ & $\begin{array}{l}\text { GO:0015031 protein } \\
\text { transport }\end{array}$ & 12,5 & 0,007 & $\begin{array}{l}\text { P62746, P55258, Q8BGY2, } \\
\text { P61028, P35279, P46638, } \\
\text { Q61595 }\end{array}$ & 4,0 & 0,327 \\
\hline $\mathrm{CC}$ & $\begin{array}{l}\text { GO:0030670 phagocytic } \\
\text { vesicle membrane }\end{array}$ & 5,4 & 0,008 & P55258, P61028, P46638 & 22,4 & 0,075 \\
\hline $\mathrm{CC}$ & GO:0005768 endosome & 10,7 & 0,018 & $\begin{array}{l}\text { P62746, P55258, Q8BU31, } \\
\text { P46638, O88983, Q5SYD0 }\end{array}$ & 3,9 & 0,129 \\
\hline $\mathrm{BP}$ & GO:0006810 transport & 19,6 & 0,033 & $\begin{array}{l}\text { P62746, P55258, Q8BGY2, } \\
\text { P61028, P35279, Q3V132, } \\
\text { P46638, P02104, O88983, } \\
\text { P62627, Q9WTR1 }\end{array}$ & 2,1 & 0,652 \\
\hline $\mathrm{CC}$ & GO:0005622 intracellular & 16,1 & 0,075 & $\begin{array}{l}\text { Q91YR1, P62746, P55258, } \\
\text { P61028, P35279, P46638, } \\
\text { P63321, P47758, Q9WTR1 }\end{array}$ & 2,0 & 0,343 \\
\hline
\end{tabular}




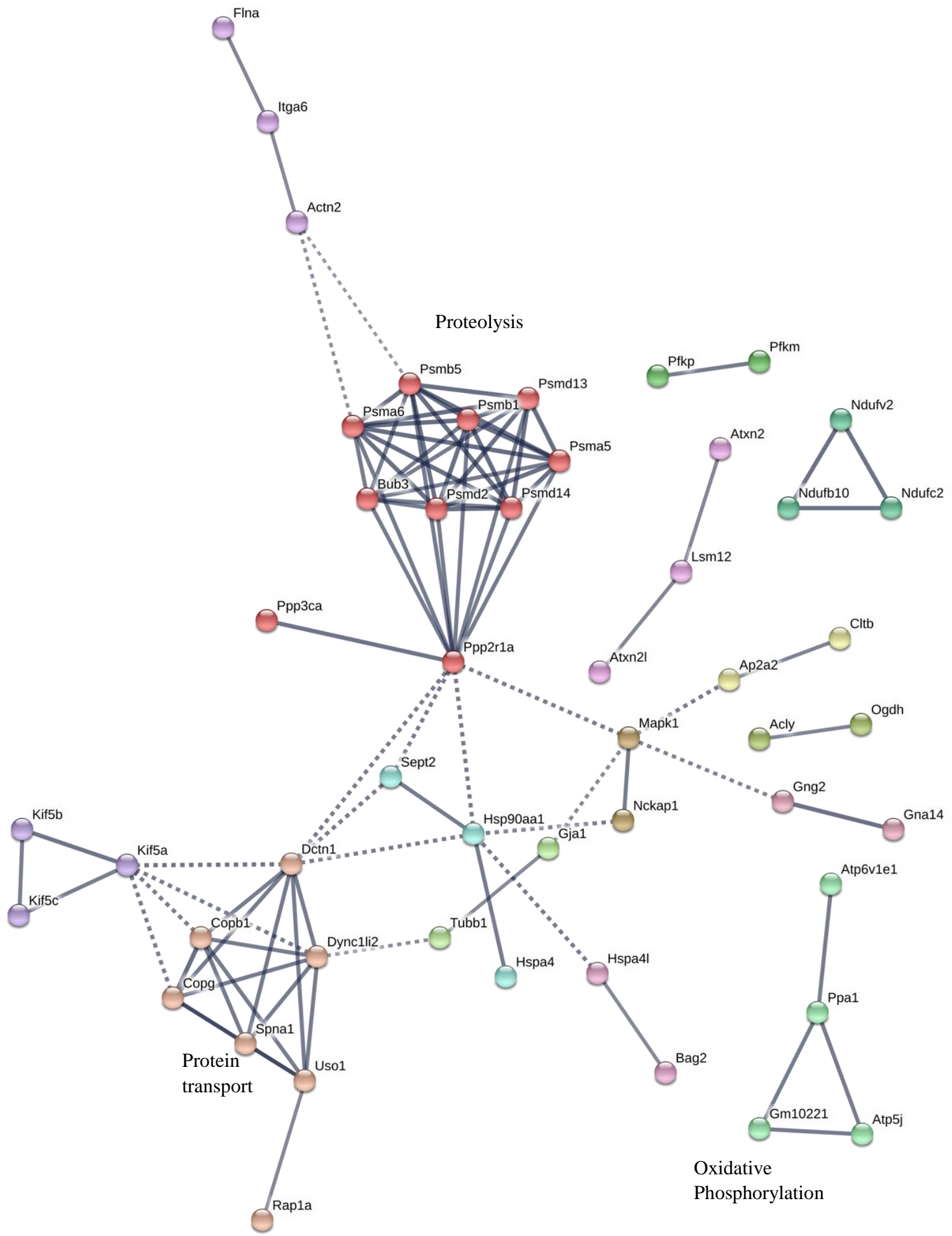

Figure 6.8: Protein-protein interaction network of Veh-enriched proteins

Figure displays known protein-protein interactions of Veh-enriched proteins and proteins only detected in Veh (denoted by gene names) as predicted with high confidence level by STRING, based on curated databases and experimental evidence (see section 2.1.7.2 for details). Unbiased MCL clustering revealed clusters (nodes are displayed in the same colour) and clusters with $>3$ nodes are annotated. Dashed lines indicated intercluster associations. Thickness of edges represent the confidence of the association. 


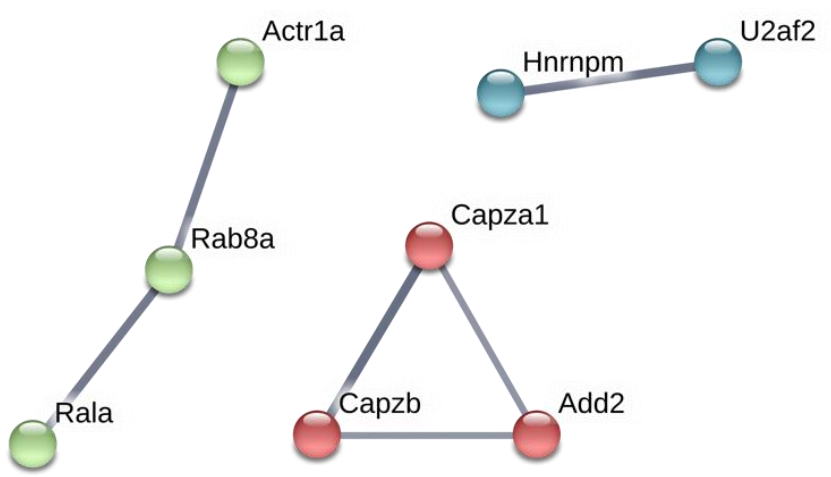

Figure 6.9: Protein-protein interaction network of CFA-enriched proteins

Figure displays known protein-protein interactions of CFA-enriched proteins and only detected in CFA (denoted by gene names) as predicted with high confidence level by STRING, based on curated databases and experimental evidence (see section 2.1.7.2 for details). Thickness of edges represent the confidence of the association. 


\title{
Curriculum Vitae
}

\author{
Julia Regina Sondermann \\ born in Bochum, Germany \\ Max-Planck Institute for Experimental Medicine \\ Somatosensory Signaling and Systems Biology Group \\ Hermann-Rein-Straße 3 \\ 37075 Göttingen, Germany \\ Tel.: +495513899578 \\ sondermann@em.mpg.de
}

\section{SCIENTIFIC EDUCATION}

10/2013 - 11/2017 Ph.D. student, Neurosciences

International Max Planck Research School (IMPRS) \&

Georg-August University, Göttingen, Germany

Thesis: Identification and characterisation of protein complexes involved in different pain states in vertebrates

Supervisor: Dr. Manuela Schmidt

Somatosensory Signaling and Systems Biology Group

Max Planck Institute for Experimental Medicine (MPIem),

Göttingen, Germany

10/2012 - 09/2013 M.Sc. student, Neurosciences

IMPRS \& Georg-August University, Göttingen, Germany

Master's thesis waived

10/2009-09/2012 B.Sc. student, Molecular Medicine

Georg-August-University, Göttingen, Germany

Thesis: Analysis of CNS Myelination in Dynamin 3-Knockout Mice

Supervisor: Prof. Dr. Mikael Simons

Cellular Neuroscience Group

MPIem, Göttingen, Germany 


\section{OTHER RESEARCH EXPERIENCE}

09/2008 - 08/2009 Voluntary Ecological Year (Freiwilliges Ökologisches Jahr)

Research assistance within several behavioural ecology and environmental research projects, mainly Attractive sinks? Breeding success of Redshanks' nesting on Wadden Sea saltmarshes of Lower Saxony (Diploma thesis, 2010, Stella M. Treffler)

Supervisor: Dr. Michael Exo

Institute of Avian Research, "Vogelwarte Helgoland", Wilhelmshaven, Germany

\section{CONFERENCE PARTicipation}

05/2015 Neurizons 2015, Göttingen, Germany

Poster: Identification and characterisation of protein complexes involved in different pain states in vertebrates

10/2015 Deutscher Schmerzkongress 2015, Mannheim, Germany Poster: Ein Proteinfingerabdruck pathologischer Schmerzen in der Maus

09/2016 $16^{\text {th }}$ World Congress on Pain, Yokohama, Japan Poster: Identification and characterization of pain-specific TRPVI interactors

\section{List of Publications}

Sondermann, J.R., Barry, A.M., Jahn, O., Michel, N., Abdelaziz, R., Gomez-Varela, D., and Schmidt, M. (2019). Vti1b promotes TRPV1 sensitization during inflammatory pain. Pain 160, 508-527.

Barry, A.M., Sondermann, J.R., Sondermann, J.-H., Gomez-Varela, D., and Schmidt, M. (2018). Regionresolved quantitative proteome profiling reveals molecular dynamics associated with chronic pain in the PNS and spinal cord. Front. Mol. Neurosci. 11, 259.

Avenali, L., Abate Fulas, O., Sondermann, J., Narayanan, P., Gomez-Varela, D., and Schmidt, M. (2017). Nocistatin sensitizes TRPA1 channels in peripheral sensory neurons. Channels 11, 11-19.

Bruderer, R.*, Sondermann, J.*, Tsou, C.C., Barrantes-Freer, A., Stadelmann, C., Nesvizhskii, A.I., Schmidt, M., Reiter, L., and Gomez-Varela, D. (2017). New targeted approaches for the quantification of data-independent acquisition mass spectrometry. Proteomics 17, 1-7.

Dembla, S., Behrendt, M., Mohr, F., Goecke, C., Sondermann, J., Schneider, F.M., Schmidt, M., Stab, J., Enzeroth, R., Leitner, M.G., et al. (2017). Anti-nociceptive action of peripheral mu-opioid receptors by Gbeta-gamma protein-mediated inhibition of TRPM3 channels. Elife 6, e26280.

Exo, K.-M., Wellbrock, A.H.J., Sondermann, J., and Maier, M. (2017). Assessing the impact of mowing on Common Redshanks Tringa totanus breeding on saltmarshes: lessons for conservation management. Bird Conserv. Int. 27, 440-453. 


\section{List of Publications (CONTINUed)}

Narayanan, P., Sondermann, J., Rouwette, T., Karaca, S., Urlaub, H., Mitkovski, M., Gomez-Varela, D., and Schmidt, M. (2016). Native Piezo2 interactomics identifies pericentrin as a novel regulator of Piezo2 in somatosensory neurons. J. Proteome Res. 15, 2676-2687.

Rouwette, T.*, Sondermann, J.*, Avenali, L., Gomez-Varela, D., and Schmidt, M. (2016). Standardized profiling of the membrane- enriched proteome of mouse dorsal root ganglia (DRG) provides novel insights into chronic pain. Mol. Cell. Proteomics 15(6), 2152-2168.

Rouwette, T., Avenali, L., Sondermann, J., Narayanan, P., Gomez-Varela, D., and Schmidt, M. (2015). Modulation of nociceptive ion channels and receptors via protein-protein interactions: Implications for pain relief. Channels 9, 175-185.

(*, equal contribution) 